\title{
GROUND-WATER DATA FOR MICHIGAN
}

\author{
BY \\ G.C. HUFFMAN
}

\begin{abstract}
U.S. GEOLOGICAL SURVEY
Open-File Report 79-332
\end{abstract}

PREPARED IN COOPERATION WITH THE MICHIGAN DEPARTMENT OF NATURAL RESOURCES GEOLOGICAL SURVEY DIVISION 

1977

GROUND-WATER DATA FOR MICHIGAN

BY

G. C. HUFFMAN

DEPARTMENT OF THE INTERIOR

U.S. GEOLOGICAL SURVEY

WATER RESOURCES DIVISION

OPEN FILE $79-332$

Prepared by the U.S. Geological Survey

in cooperation with the

State of Michigan

Department of Natural Resources

H. A. Tanner, Director

Geological Survey Division

A. E. Slaughter, State Geologist 
UNITED STATES DEPARTMENT OF THE INTERIOR

CECIL D. ANDRUS, Secretary

GEOLOGICAL SURVEY

H. William Menard, Director

For additional information write to:

Office of the District Chief

Water Resources Division

U.S. Geological Survey

6520 Mercantile Way, Suite 5

Lansing, Michigan 48910 
Introduction - 1

Purpose of this report

What this report contains - 1

Uses of data in this report

How records can be obtained -.... 3

Other ground-water reports

We11-numbering system

Ground-water levels in 1977

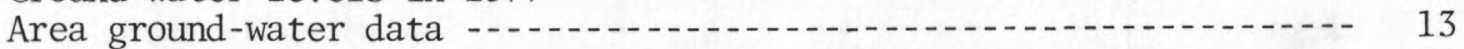

Branch County

- City of Coldwater -...... 15

Calhoun County - City of Battle Creek -........-. 16

Clinton County - City of St. Johns -...- 17

Eaton County - Delta Township -

Gratiot County - City of St. Louis -.... 19

Ingham County - City of Lansing -..... 20

- City of Mason -................. 21

- East Lansing-Meridian Water Authority ----- 22

- Lansing Township -..--

- Michigan State University -...-_..-_..- 24

Jackson County - City of Jackson -

Kalamazoo County - City of Kalamazoo -................. 26

- City of Portage -.... 27

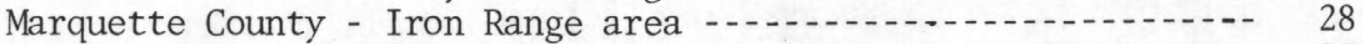

Oakland County - Huron-Clinton Metropolitan Authority --.---- 29

Van Buren County - 30

Washtenaw County - City of Ann Arbor -....... 31

- City of Ypsilanti -........ 32

- Ypsilanti Township -........ 33

Selected references - 69

Acknowledgments - 74 


\section{FIGURES}

Page

1. Map showing distribution of observation wells _............ 2

2. Map showing areas where ground-water conditions are described in published reports in the Upper Peninsula .....- 4

3. in the Lower Peninsula -..... 6

4. Map showing deviation of precipitation from normal _........ 11

5. Graphs of water levels in selected wells

\section{TABLES}

1. Observation wells

2. Pumpage - 40

3. Quality - 46

\section{METRIC EQUIVALENTS}

Conversion Factors

The following factors may be used to convert the English units published in this report to the International System of Units (SI).

\begin{tabular}{|c|c|c|}
\hline Multiply English units & By & To obtain SI units \\
\hline acres (a) & 0.4047 & hectares (ha) \\
\hline feet $(\mathrm{ft})$ & .3048 & meters (m) \\
\hline inches (in) & 25.4 & millimeters (mm) \\
\hline miles (mi) & 1.609 & kilometers $(\mathrm{km})$ \\
\hline million gallons ( $10^{6}$ gal) & 3,785 & cubic meters $\left(\mathrm{m}^{3}\right)$ \\
\hline ga1lons (ga1) & 3.785 & 1iters (1) \\
\hline
\end{tabular}


GROUND-WATER DATA FOR MICHIGAN

by

G. C. HUFFMAN

INTRODUCTION

Purpose of this report

The purpose of this report is to make available the 1977 records of water levels and related data for the principal aquifers of the State. These records and data provide a means for evaluating available ground-water supplies. Longterm records serve as a framework to which short-term records may be related. Also, water levels in areas of heavy pumping may be compared to levels in areas of little or no pumping.

This report is written for municipalities, industries, institutions, consultants, drillers, hydrologists, and other people interested in the groundwater resources of the State.

What this report contains

Data on the yield of wells, pumpage, quality of water, and trends of groundwater levels for the past 5 years are shown in the text. Many hydrographs are included to illustrate changes in water levels. Yield data are given as they were reported by water departments and consultants. Also included are data on municipal, public, and industrial water-supply facilities.

Tables 1, 2, and 3 contain records of water levels in observation we1ls, well locations, depths, altitudes, aquifers tapped, water-level extremes, records of pumpage by most major ground-water users in the State, and water quality data from selected wells sampled during 1975-77. Figure 1 shows distribution of observation wells in the State.

\section{Uses of data in this report}

An effective method of determining the amount of water available from an aquifer is the analysis of records of water levels and pumpage. Many water level records in pumped areas are obtained by means of recorders. These records serve to indicate day-to-day and long-term effects of pumping. This information can be used by municipalities, industries, and institutions to estimate the capacity of aquifers to meet present and future demands for water and whether expansion of present ground-water supply systems is practicable. 


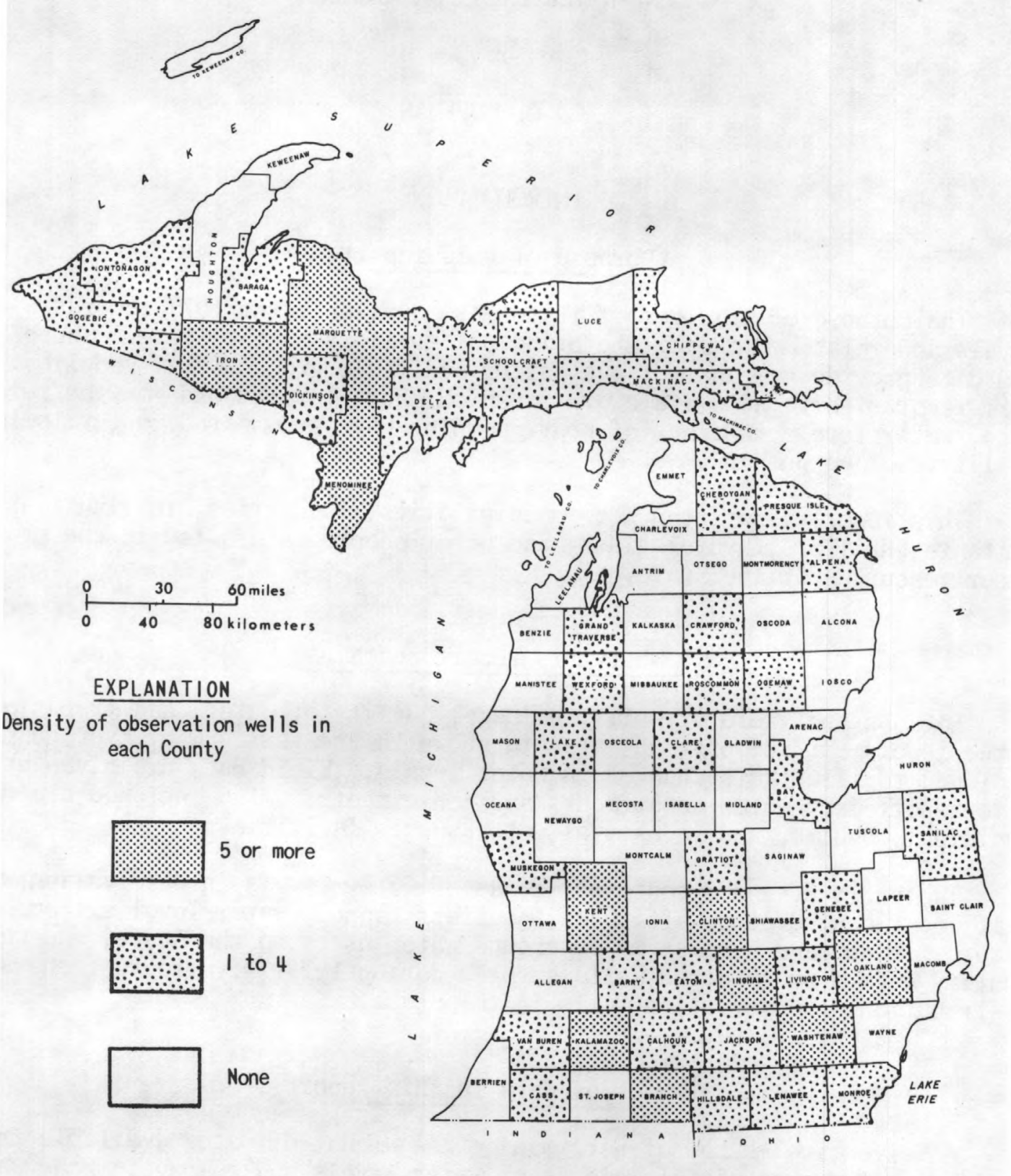

Figure 1.--Distribution of observation wells. Water levels were monitored in 157 observation we11s in 1977. 
When new wells are to be installed in an area where water levels are declining because of pumping, projections of future water levels will indicate the depth below which the intake should be installed. Allowing for probable lowering of water levels can extend the life of the installation.

Water problems often are encountered when a basement or septic tank is constructed for a building or home. The water table fluctuates an average of 2 to 3 feet $(0.6$ to $0.9 \mathrm{~m})$ annually and about 5 feet $(1.5 \mathrm{~m})$ over a period of years. Thus, if an excavation is made in the fall, when the water table is low, good construction practices would allow for the probable higher water levels in the spring. If construction is made after several years of drought, the allowance for the subsequent rise in water levels would be larger. If a site is at all questionable, borings would be made to determine the depth to water table, and allowances for the probable rise in water levels could be made.

How records can be obtained

Complete tabulations of water-leve1 measurements, hydrographs for observation wells, records of chemical quality, water-temperature measurements, well records and logs, aquifer tests, records of pumping for public and industrial supplies, and water-resources reports are on file for public inspection. They may be examined at the Geological Survey Division, Michigan Department of Natural Resources, Mason Building, Lansing, Michigan 48909; or at the U.S. Geological Survey, 6520 Mercantile Way, Suite 5, Lansing, Michigan 48910. Records for the Northern Peninsula are also kept on file in the State and Federal Geological Survey Offices, State Office Building, Escanaba, Michigan 49829.

\section{Other ground-water reports}

From 1935 to 1974, records of ground-water levels in Michigan were published in U.S. Geological Survey Water-Supply Papers (WSP), as shown on page 72. Since 1975, these records have been published in U.S. Geological Survey Water-Data Reports (WDR).

To supplement the Water-Supply Paper and Water-Data Report series, publications of annual reports, titled "Summary of Ground-Water Conditions in Michigan," was begun in 1956. Beginning in 1967, the title of the reports was changed to "Summary of Ground-Water Hydrological Data in Michigan," and in 1973 to "Ground-Water Data for Michigan."

Reports that describe ground water in Michigan are shown in figures 2 and 3. In addition, many publications dealing with ground water are 1 isted in the selected references at the end of this report. 


\section{EXPLANATION}

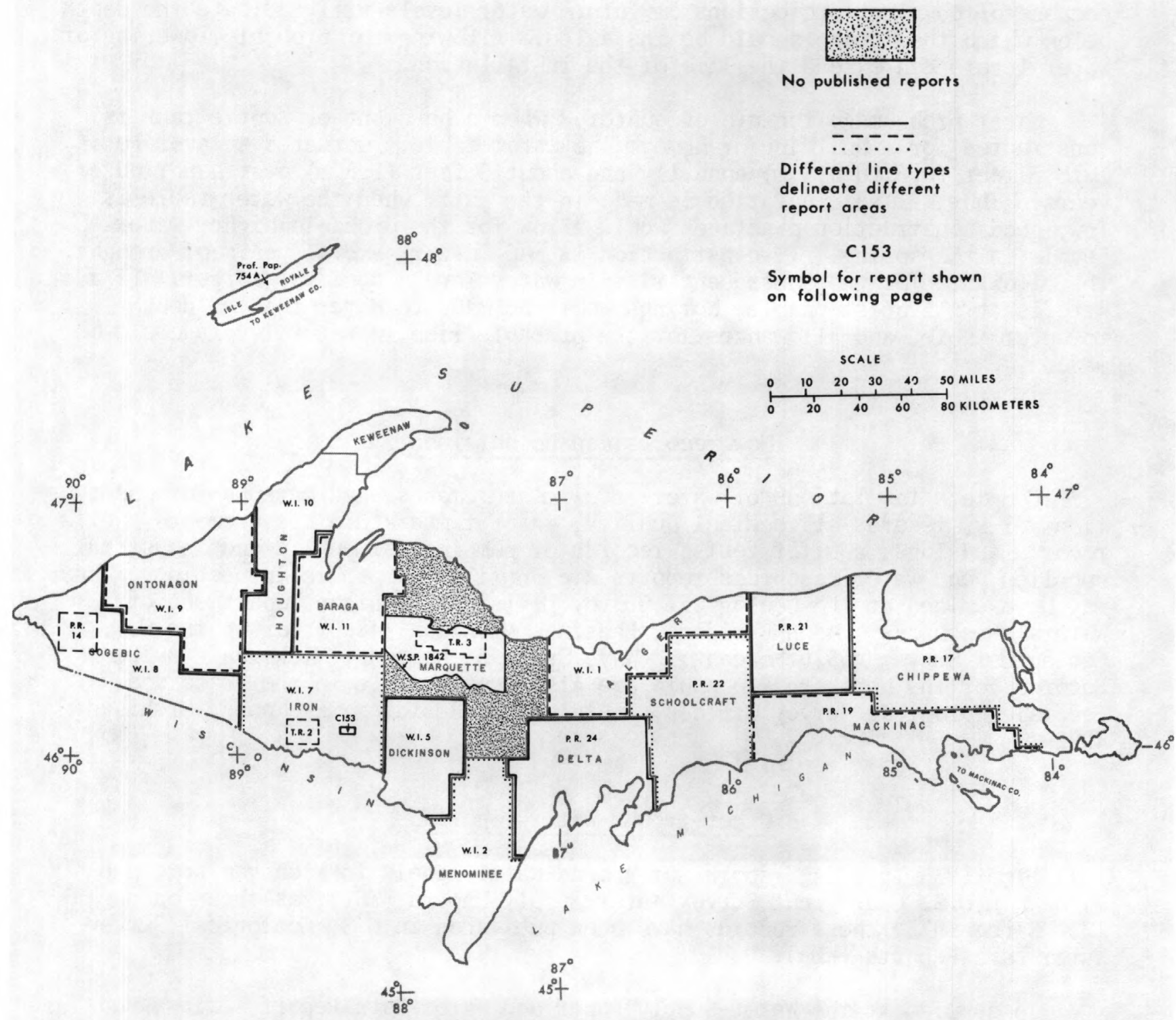

Figure 2.--Areas in the Upper Peninsula where ground-water conditions are described in published reports. 
Prof. Pap. 754A -- Huber, N. K., 1973, Glacial and postglacial geologic history of Isle Royale National Park, Michigan: U.S. Geol. Survey Prof. Paper 754-A.

\section{Progress Reports}

PR 14 -- Brown, E. A., and Stuart, W. T., 1951, Ground-water resources of the glacial deposits in the Bessemer area, Michigan: Michigan Geoi. Survey Prog. Rept. 14.

PR 17 -- Vanlier, K. E., and Deutsch, Morris, 1958, Reconnaissance of the ground-water resources of Chippewa County, Michigan: Michigan Geol. Survey Prog. Rept. 17.

PR 19 -- 1958, Reconnaissance of the ground-water resources of Mackinac County, Michigan: Michigan Geol. Survey Prog. Rept.' 19.

PR 21 -- Vanlier, K. E., 1959, Reconnaissance of the ground-water resources of Luce County, Michigan: Michigan Geol. Survey Prog. Rept. 21 .

PR 22 -- Sinclair, W. C., 1959, Reconnaissance of the ground-water resources of Schoolcraft County, Michigan: Michigan Geol. Survey Prog. Rept. 22.

PR 24 -- Rept. 1960, Reconnaissance of the ground-water resources of Delta County, Michigan: Michigan Geo1. Survey Prog.

\section{Technical Reports}

TR 2 -- Stuart, W. T., Theis, C. V., and Stanley, G. M., 1948, Ground-water problems in the Iron River district, Michigan: Michigan Geol. Survey Tech. Rept. 2.

TR 3 -- Stuart, W. T., Brown, E. A., and Rhodehame1, E. C., 1954, Ground-water investigations of the Marquette iron-mining district, Michigan: Michigan Geol. Survey Tech. Rept. 3 .

\section{Water Investigations}

WI 1 -- Vanlier, K. E., 1963, Ground water in Alger County: Michigan Geol. Survey Water Inv. 1.

WI 2 -- __ 1963, Ground water in Menominee County: Michigan Geol. Survey Water Inv. 2.

WI 5 -- Hendrickson, G. E., and Doonan, C. J., 1966, Ground-water resources of Dickinson County, Michigan: Michigan Geol. Survey Water Inv, 5

WI 7 -- Doonan, C. J., Hendrickson, G. E., 1967, Ground water in Iron County, Michigan: Michigan Geol. Survey Water Inv. 7.

WI 8 -, 1968, Ground water in Gogebic County, Michigan: Michigan Geol: Survey Water Inv. 8.

WI 9 -.__ 1968, Ground water in Ontonagon County, Michigan: Michigan Geol. Survey Water Inv. 9.

WI 10 -- Doonan, C. J., Hendrickson, G. E., and Byerlay, J. R., 1970, Ground water and geology of Keweenaw Peninsula, Michigan: Michigan Geol. Survey Water Inv, 10.

WI 11 -- Doonan, C. J., and Byerlay, J. R., 1973, Ground water and geology of Baraga County, Michigan: Michigan Geol. Survey Water Inv. 11 .

\section{Water-Supply Papers}

WSP 1842 -- Wiitala, S. W., Newport, T. G., and Skinner, E. L., 1967, Water resources of the Marquette Iron Range area, Michigan: U.S. Geol. Survey Water-Supply Paper 1842. 


\section{EXPLANATION}

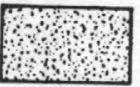

No published reports

$\overline{-\ldots-.-1}$

Different line types delineate different report areas

C183

Symbol for report shown on following page

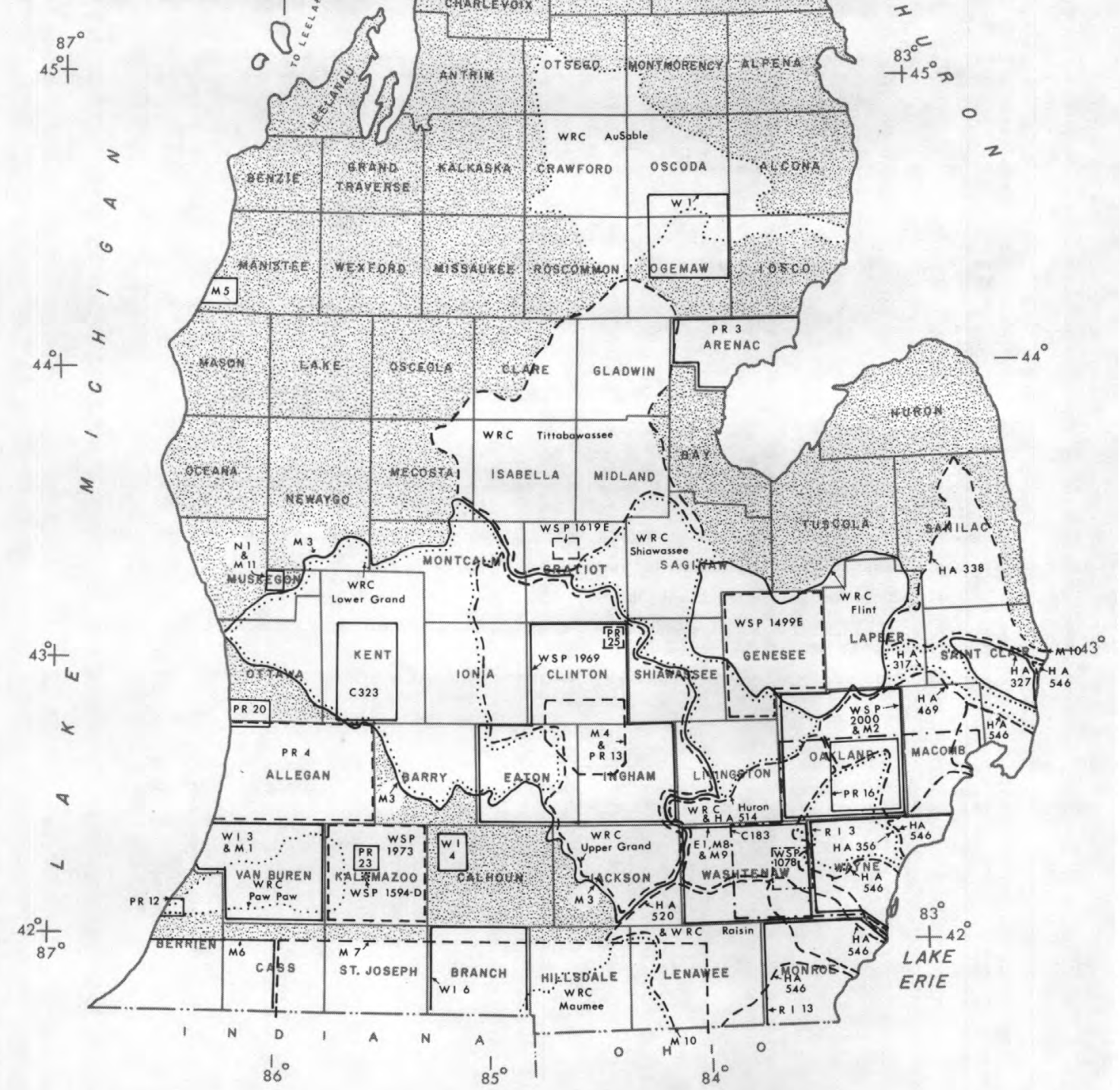

Figure 3.--Areas in the Lower Peninsula where ground-water conditions are described in published reports. 
C 183 -- Wisler, C. O., Strame1, G. J., and Laird, L. B., 1952, Water resources of the Detroit area, Michigan: U.S. Geol. Survey Circ. 183 .

C 323 -- Stramel, G. J., Wisler, C. O., and Laird, L. B., 1954, Water resources of the Grand Rapids area, Michigan: U.S. Geol. Survey Circ. 323.

\section{Environmental Geology Series Reports}

E 1 -- Fleck, W. B., 1974, Geology and hydrology for environmental planning in Washtenaw County, Michigan: Michigan Geol. Survey Environmental Geol. Series Rept. 1.

\section{Hydrologic Atlases}

HA 317 -- Knutilla, R, L., 1969, Water resources of the Belle River basin, southeastern Michigan: U.S. Geol. Survey Hydrol, Inv. Atlas HA-317.

HA 327 -- ___ 1969, water resources of the Pine River basin, southeastern Michigan: U.S. Geo1. Survey Hydrol. Inv. At1as HA-327.

HA 338 -- ___ 1970, Water resources of the Black River basin, southeastern Michigan: U.S. Geol. Survey Hydrol. Inv. At1as HA-338. HA 356 -- __ 1971, Water resources of the River Rouge basin, southeastern Michigan: U.S. Geo1. Survey Hydrol. Inv. Atlas HA-356. HA 469 -- Nowlin, J. O., 1973, Water resources of the Clinton River basin, southeastern Michigan: U.S. Geol. Survey Hydro1. Inv.
Atlas HA-469.

HA 514 -- Larson, R. W., Allen, W. B., and Hanson, S. D., 1975, Water resources of the Huron River basin, southeastern Michigan: U.S. Geol. Survey Hydrol. Inv. Atlas HA-514.

HA 520 -- Knutilla, R. L., and Allen, W. B., 1975, Water resources of the River Raisin basin, southeastern Michigan: U.S. Geol. Survey Hydrol. Inv. Atlas HA- 520 .

HA 546 -- Twenter, F. R., Knutilla, R. L., Cummings, T. R., 1975, Water resources of basins for minor streams draining into St. Clair River, Lake St. Clair, Detroit River, and Lake Erie, southeastern Michigan: U.S. Geol. Survey Hydro1. Inv. At1as HA-546.

\section{Miscellaneous Reports}

M 1 -- Terwilliger, F. W., 1954, The glacial geology and ground-water resources of Van Buren County, Michigan, pt. 1 of Occasional papers for 1954 on the geology of Michigan: Michigan Geo1. Survey Pub. 48.

M 2 -- Mozola, A. J., 1953, A survey of ground-water resources in Oakland County, Michigan, pt. 2 of Occasional papers for 1954 on the geology of Michigan: Michigan Geol. Survey Pub, 48.

M 3 -- Vanlier, K. E., 1968, Appendix E of the report on the Grand River Comprehensive Basin Study: U.S. Army Eng. District, Detroit, Michigan.

M 4 -- Vanlier, K. E., and Wheeler, M. L., 1968, Analog simulation of ground-water development of the Saginaw Formation, Lansing metropolitan area, Michigan! Tri-County Planning Commission, Lansing Ground-Water Rept.

M 5 -- Childs, K. E., 1970, History of the salt, brine, and paper industries and their probable effect on the ground-water quality in the Manistee Lake area, Michigan: Michigan Dept. Nat. Resources.

M 6 -- Schneider, A. F., and Keller, S. J., 1970, Indiana Geological Survey regional geological map number 4: Indiana Dept. Nat. Resources.

M 7 -- Johnson, G. H., and Keller, S. J., 1972, Indiana Geological Survey regional geological map number 8: Indiana Dept. Nat. Resources.

M 8 -- Twenter, F. R., Knutilla, R. L., and Nowlin, J. 0., 1976, Water resources of Washtenaw County, Michigan: Washtenaw County Metropolitan Plan. Comm.

M 9 -- Borton, T. E., 1974, Planning perspectives on water resources, Washtenaw County, Michigan: Washtenaw County Metropolitan Plan. Comm.

M 10 -- Twenter, F. R., 1975, Ground water and geology -- southeastern Michigan; U.S. Army Corps of Engineers.

M 11 -- Fleck, W. B., and McDonald, M. G., 1978, Three-dimensional finite-difference model of ground-water system underlying the Muskegon County wastewater disposal system, Michigan: U.S. Geol. Survey Jour. of Research, v. 6, no. 3.

\section{Open-File Reports}

N 1 -- McDonald, M. G., and Fleck, W. B., 1978, Model analysis of the impact on ground-water conditions of the Muskegon County wastewater disposal system, Michigan: U.S. Geol. Survey open-file rept. 78-99.

\section{Progress Reports}

PR 3 -- Pringle, G. H., 1937, Geology of Arenac County, Michigan: Michigan Geol. Survey Prog. Rept. 3.

PR 4 -- Riggs, C. H., 1938, Geology of Allegan County, Michigan: Michigan Geo1. Survey Prog. Rept. 4.

PR 12 -- Stuart, W. T., and Stallman, R. W., 1945, Ground-water resources of the Benton Harbor area, Michigan: Michigan Geol. Survey Prog. Rept. 12. 
PR 13 -- Stuart, W. T., 1945, Ground-water resources of the Lansing area, Michigan: Michigan Geol. Survey Prog. Rept. 13.

PR 16 -- Ferris, J. G., and others, 1954, Ground-water resources of southeastern Oakland County, Michigan: Michigan Geol. Survey Prog. Rept. 16.

PR 20 -- Deutsch, Morris, Burt, E. M., and Vanlier, K. E., 1958, Summary of ground-water investigations in the Holland area, Michigan: Michigan Geol. Survey Prog. Rept. 20.

PR 23 -- Deutsch, Morris, Vanlier, K. E., and Giroux, P. R., 1960, Ground-water hydrology and glacial geology of the Kalamazoo area, Michigan: Michigan Geol. Survey Prog. Rept. 23

PR 25 -- Vanlier, K. E., 1962, Summary of ground-water investigations in the Elsie area, Michigan: Michigan Geol. Survey Prog. Rept. 25.

\section{Reports of Investigations}

RI 3 -- Mazola, A. J., 1969, Geology for 1and and ground-water development in Wayne County, Michigan: Michigan Geol. Survey Rept. RI 13 -, 1970, Geology for environnental planning in Monroe County, Michigan: Michigan Geol. Survey Rept. Inv. 13.

\section{Water Information Series}

W 1 -- Knutilla, R. L., Twenter, F. R., and Larson, R. W., 1971, Upper Rifle River Basin -- An Evaluation of its Water Resources and Hydrologic Environment: Michigan Geol. Survey Water Information Series Rept. 1.

\section{Water Investigations}

WI 3 -- Giroux, P. R., Hendrickson, G. E., Stoimenoff, L. E., and Whetstone, G. W., 1964, Water resources of Van Buren County, Michigan: Michigan Geol, Survey Water Inv, 3.

WI 4 -- Vanlier, K. E., 1966, Ground-water resources of the Battle Creek area, Michigan: Michigan Geo1. Survey Water Inv. 4.

WI 6 -- Giroux, P. R., Stoimenoff, L. E., Nowlin, J. O., and Skinner, E. L., 1966, Water resources of Branch County, Michigan: Michigan Geol. Survey Water Inv.'6.

\section{Michigan Water Resources Commission Reports}

WRC Au Sable -- Water resource conditions and uses in the Au Sable River Basin, 1966: Michigan Water Resources Comm. Rept.

WRC Flint -- Water resource conditions and uses in the Flint River Basin, 1956: Michigan Water Resources Comm. Rept.

WRC Huron -- Water resource conditions and uses in the Huron River Basin, 1957: Michigan Water Resources Comm. Rept.

WRC Lower Grand -- Water resource conditions and uses in the Lower Grand River Basin, 1967, (open file): Michigan Water Resources Comm. Rept.

WRC Maumee -- Water resource conditions and uses in the Maumee River Basin, 1964: Michigan Water Resources Corm. Rept.

WRC Paw Paw -- Water resource conditions and uses in the Paw Paw River Basin, 1955, (revised report in 1964): Michigan Water Resources Comm. Rept.

WRC Raisin -- Water resource conditions and uses in the River Raisin Basin, 1965: Michigan Water Resources Corm. Rept.

WRC Shiawassee -- Water resource conditions and uses in the Shiawassee River Basin, 1963: Michigan Water Resources Comm. Rept.

WRC Tittibawassee -- Water resource conditions and uses in the Tittibawassee River Basin, 1960: Michigan Water Resources Comm. Rept.

WRC Upper Grand -- Water resource conditions and uses in the Upper Grand River Basin, 1961: Michigan Water Resources Corm. Rept.

\section{Water Supply Papers}

WSP 1078 -- McGuinness, C. L., Poindexter, O. F., and Otten, E. G., 1949, Ground-water supplies of the Ypsilanti area, Michigan: U.S. Geol. Survey Water-Supply Paper 1078

WSP 1499E -- Wiitala, S. W., Vanlier, K. E., and Krieger, R. A., 1960, Water resources of the Flint area, Michigan: U.S. Geol. Survey Water-Supply Paper 1499-E.

WSP 15941) -- Reed, J. E., Deutsch, Morris, and Wiitala, S. W., 1966, Induced recharge of an artesian glacial-drift aquifer at Kalamazoo, Michigan: U.S. Geol. Survey Water-Supply Paper 1594-D.

WSP 1619E -- Vanlier, K. E., 1963, Ground-water resources of the Alma area, Michigan: U.S. Geol. Survey Water-Supply Paper 1619-E.

WSP 1969 -- Vanlier, K. E., Wood, W. W., and Brunett, J. O., 1973, Water-supply development and management alternatives for C1inton, Eaton, and Ingham Counties, Michigan: U.S. Geol. Survey Water-Supply Paper 1969.

WSP 1973 -- Allen, W. B., Miller, J. B., and Wood, W. W., 1972, Availability of water in Kalamazoo County, Michigan: U.S. Geol. Survey Water-Supply Paper 1973

WSP 2000 -- Twenter, F. R., and Knutilla, R. L., 1972, Water for a rapidly growing urban community -- Oakland County, Michigan: U.S. Geol. Survey Water-Supply Paper 2000. 
The well-numbering system for Michigan indicates the location of wells within the rectangular subdivision of the land with reference to the Michigan meridian and base line. The first two segments of the well number designate township and range, the third segment of the number designates the section and the letters A thru D designate successively smaller subdivisions of the section as shown below. Thus, a well designated as 32N 6E 16CCCB would be located to the nearest 2.5 acres ( 1 hectare) and would be within the shaded area in section 16.

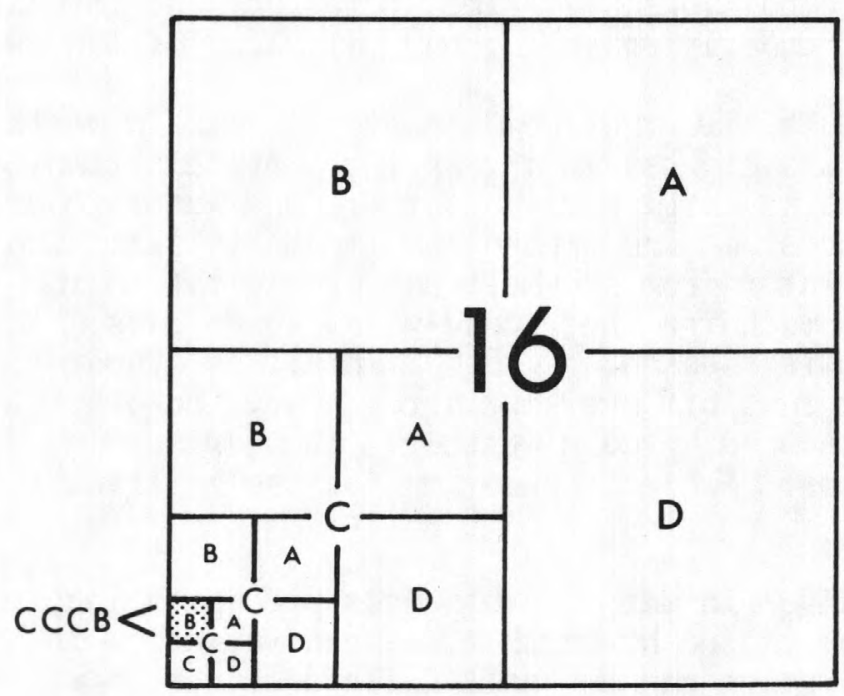

For many wells in this report, locations are only given to the nearest 40 -acre (16 hectares) tract, for example, 16CC. In the event that two or more wells are located in the same tract, a sequential number designation is added-for example, $16 \mathrm{CC} 1,16 \mathrm{CC} 2,160 \mathrm{C} 3$, etc. The Michigan Geological Survey uses a similar system except that numbers are used in lieu of letters. 
Measurements of water levels were made in 157 observation wells (table 1). Record low levels occurred in 31 of the wells despite above normal precipitation in most climatological regions (fig. 4). Only 3 wells were at record high levels, compared with 32 in 1976. Levels were generally below average in many wells during the year; however, most wells had returned to near normal by years end (fig. 5).

Although precipitation is one of the major factors affecting ground-water levels, annual total rainfall may not always bear a direct relationship to the amount of recharge received by an aquifer. Many factors affect this relationship, such as (1) soil conditions; (2) time, duration, and intensity of precipitation; (3) nature of underlying rocks; and (4) slope of land surface.

Hydrographs of natural fluctuations of water levels in wells (fig. 5) show that water levels are highest in the spring. At this time, snowmelt and rain normally result in large additions to ground-water reservoirs. However, ice cover or frost in the ground can impede infiltration. Under these conditions, most water from snowmelt and precipitation runs off rapidly, and very little goes to recharge the ground-water reservoirs. There is little recharge during the growing season, as most rainfall is evaporated, is transpired by vegetation, or runs off overland after heavy showers. In the fall, evapotranspiration is reduced by cold weather. Thus, rises in water levels usually follow fall rains. Frozen ground impedes the infiltration of water during the winter.

In addition to changes in water levels from precipitation, temporary changes in levels may be caused by earth tides and variation in barometric pressure. Evapotranspiration causes sma1l daily declines in water levels in some wells. 


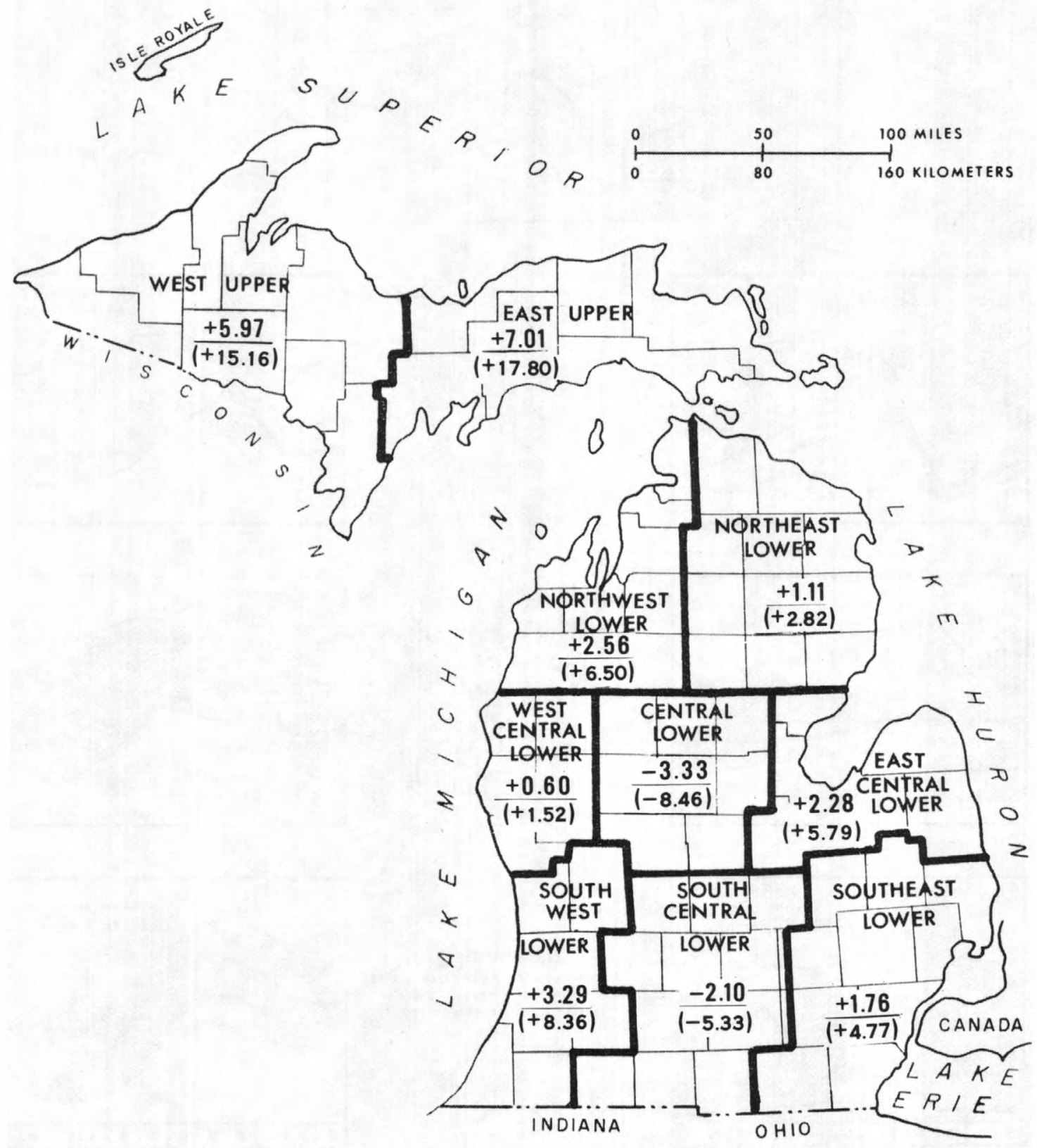

Figure 4.--Deviation of precipitation from normal during 1977 by climatological regions. Figures are in inches and, centimeters in parenthesis. Normals are based on data for 1931-60. 


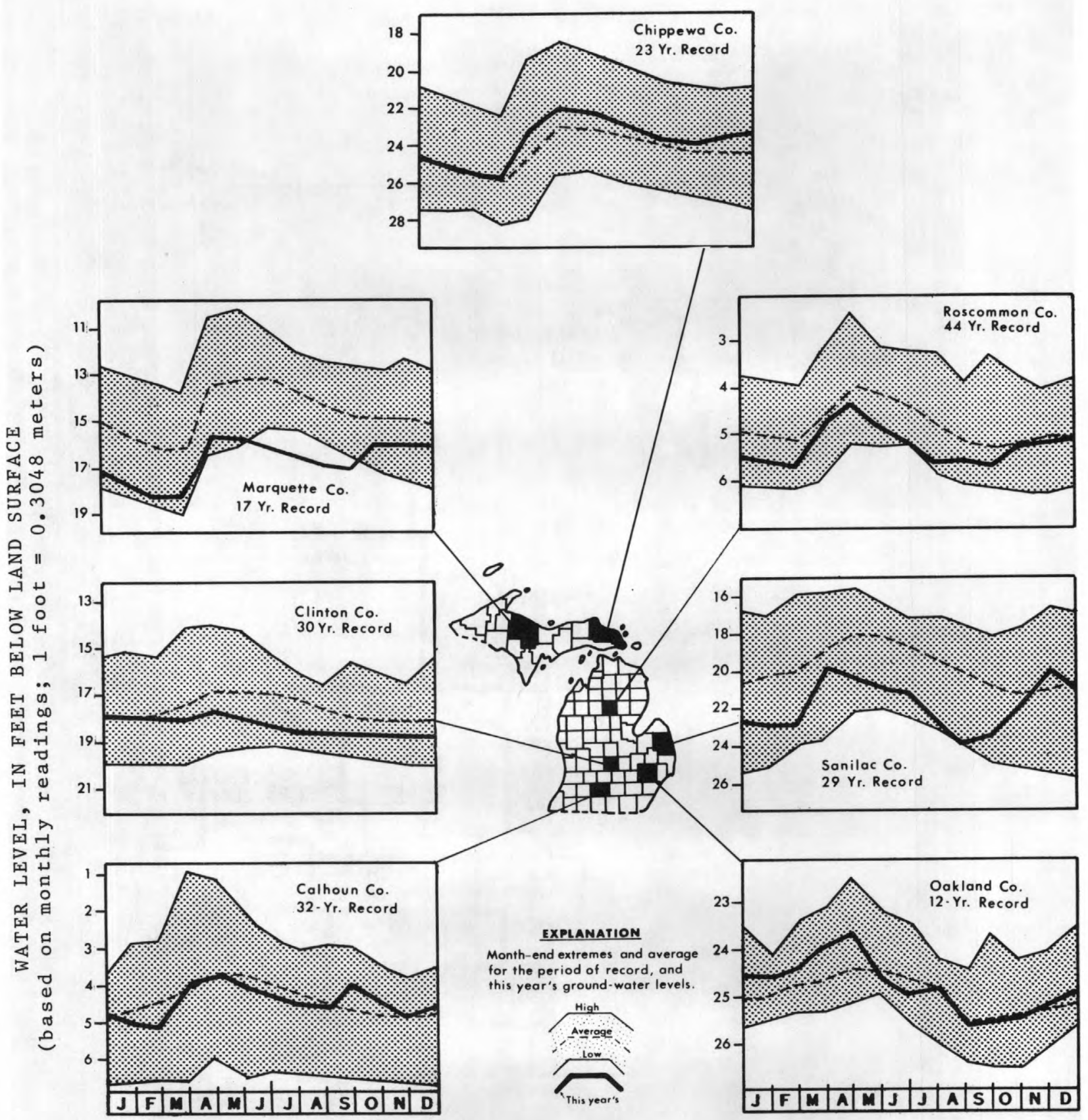

Figure 5.--Water levels in selected wells during 1977. 


\section{AREA GROUND-WATER DATA}

Descriptions of some of Michigan's municipal, institutiona1, and area1 ground-water supplies follow alphabetically, by counties. Included are data on the chemical quality of water, based on latest analyses made by the Michigan Department of Health. Where more than one well is involved, a range in quality is generally given. In this report, the unit milligrams per liter (mg/L) can be considered to be numerically equal to parts per million (ppm) . Most descriptions are supplemented by illustrations. 

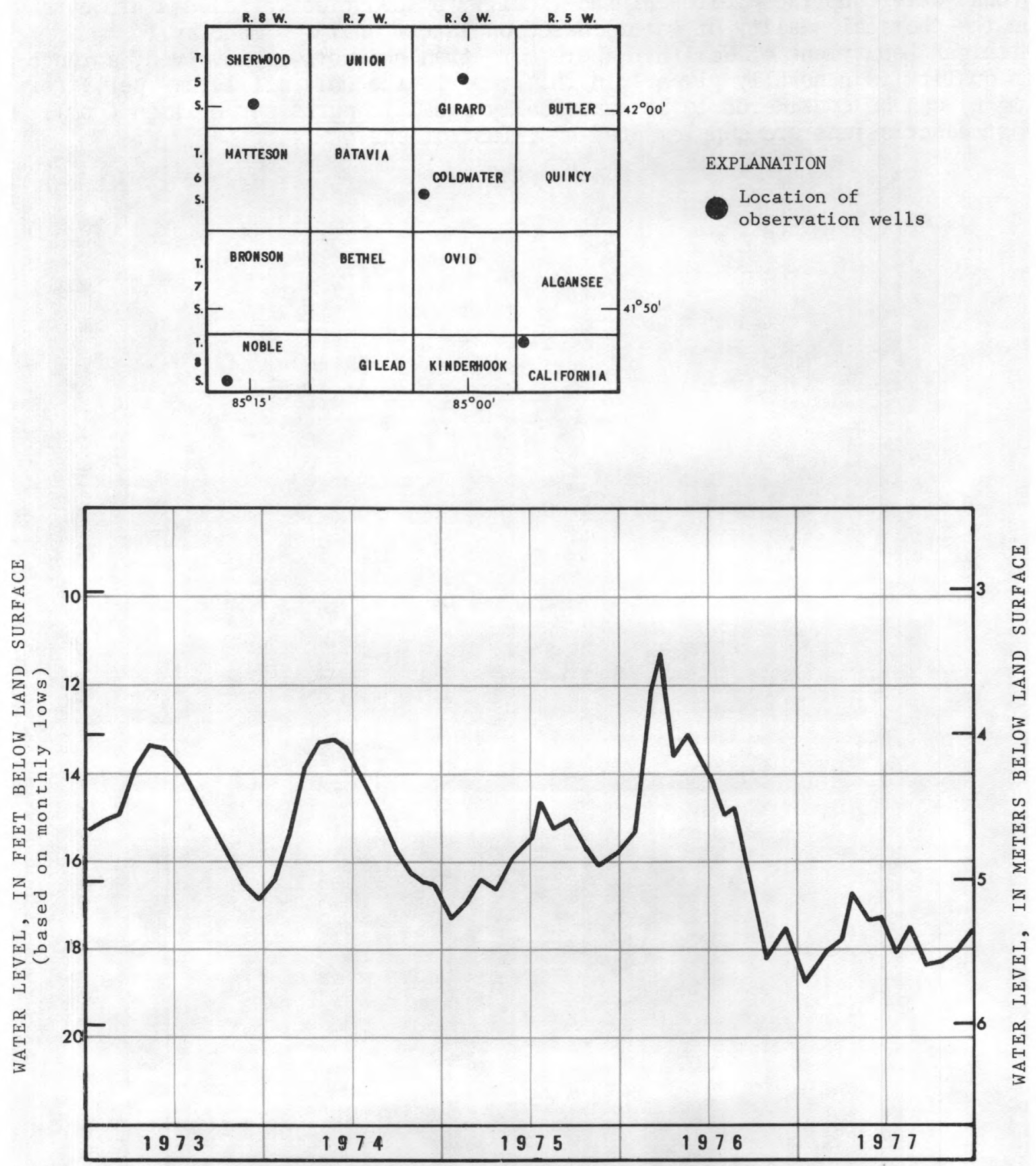

Water levels in observation we11 5S $8 \mathrm{~W}$ 28DB in Branch County. We11 is 42 feet deep and in glacial deposits. Levels shown are typical of observation wells located in the county. 
SUPPLY AND SOURCE -- 4 we1ls, 121 to 132 feet deep, tap the glacial drift. YIELD OF WELLS -- No. 3 - 1,200; No. 4 - 1,400; No. 5 - 2,250; No. 6 2,850 $\mathrm{gal} / \mathrm{min} ;$ specific capacity - 80 to $190 \mathrm{gal} / \mathrm{min} / \mathrm{ft}$ of $\mathrm{drawdown}$.

PUMPAGE -- Total annual pumpage, in million gallons, for past 5 years.

$$
\begin{array}{r}
1977-1,154 \\
1976-1,085 \\
1975-965 \\
1974-1,024 \\
1973-1,023
\end{array}
$$

$$
\begin{array}{ll}
\text { QUALITY OF WATER -- } & \text { Hardness } \\
\text { Iron } & 300-330 \mathrm{mg} / \mathrm{L} \\
& \text { Diss. Solids } 343-390 \mathrm{mg} / \mathrm{L}
\end{array}
$$

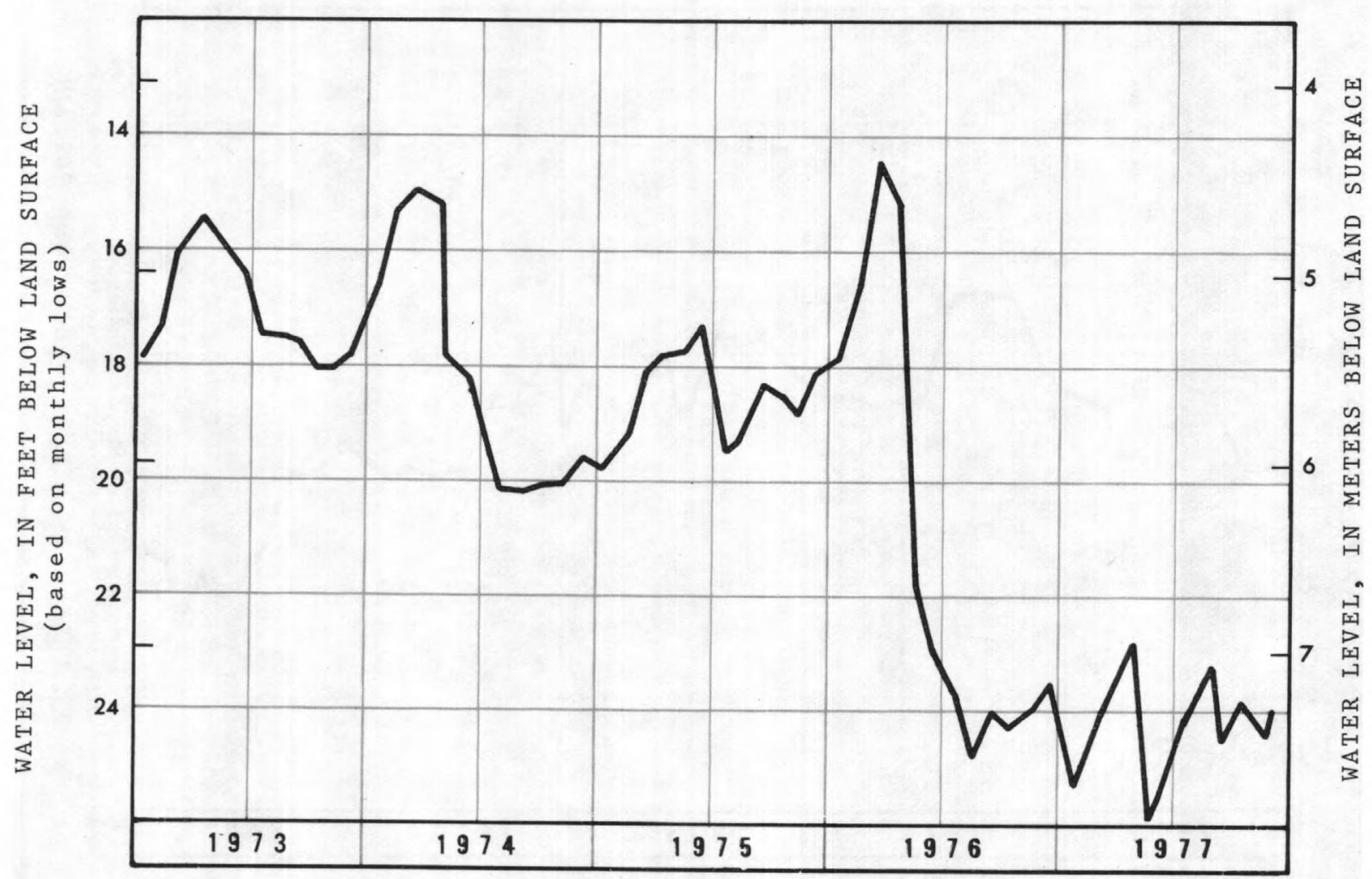

Water levels in observation we11 6S 6W 22CA at Coldwater. We11 is 113 feet deep and in glacial deposits. 


\section{CALHOUN COUNTY - CITY OF BATTLE CREEK}

SUPPLY AND SOURCE - - 29 we11s, 120 to 160 feet deep, tap sandstones of the Marshall Formation. All are located at the Verona Field.

YIELD OF WELLS -- 300 to $1,000 \mathrm{ga} 1 / \mathrm{min}$; specific capacity -- 50 to 650 $\mathrm{gal} / \mathrm{min} / \mathrm{ft}$ of drawdown.

PUMPAGE - - Total annual pumpage, in million gallons, for past 5 years.

$$
\begin{aligned}
& 1977-2,443 \\
& 1976-2,357 \\
& 1975-2,224 \\
& 1974-2,168 \\
& 1973-2,685
\end{aligned}
$$

$$
\begin{array}{lr}
\text { QUALITY OF WATER -- } & \text { Hardness } \\
& \text { Iron } \\
& \text { Diss. Solids } \begin{aligned}
245-345 \mathrm{mg} / \mathrm{L} \\
0.05-1.3 \mathrm{mg} / \mathrm{L} \\
300-413 \mathrm{mg} / \mathrm{L}
\end{aligned}
\end{array}
$$

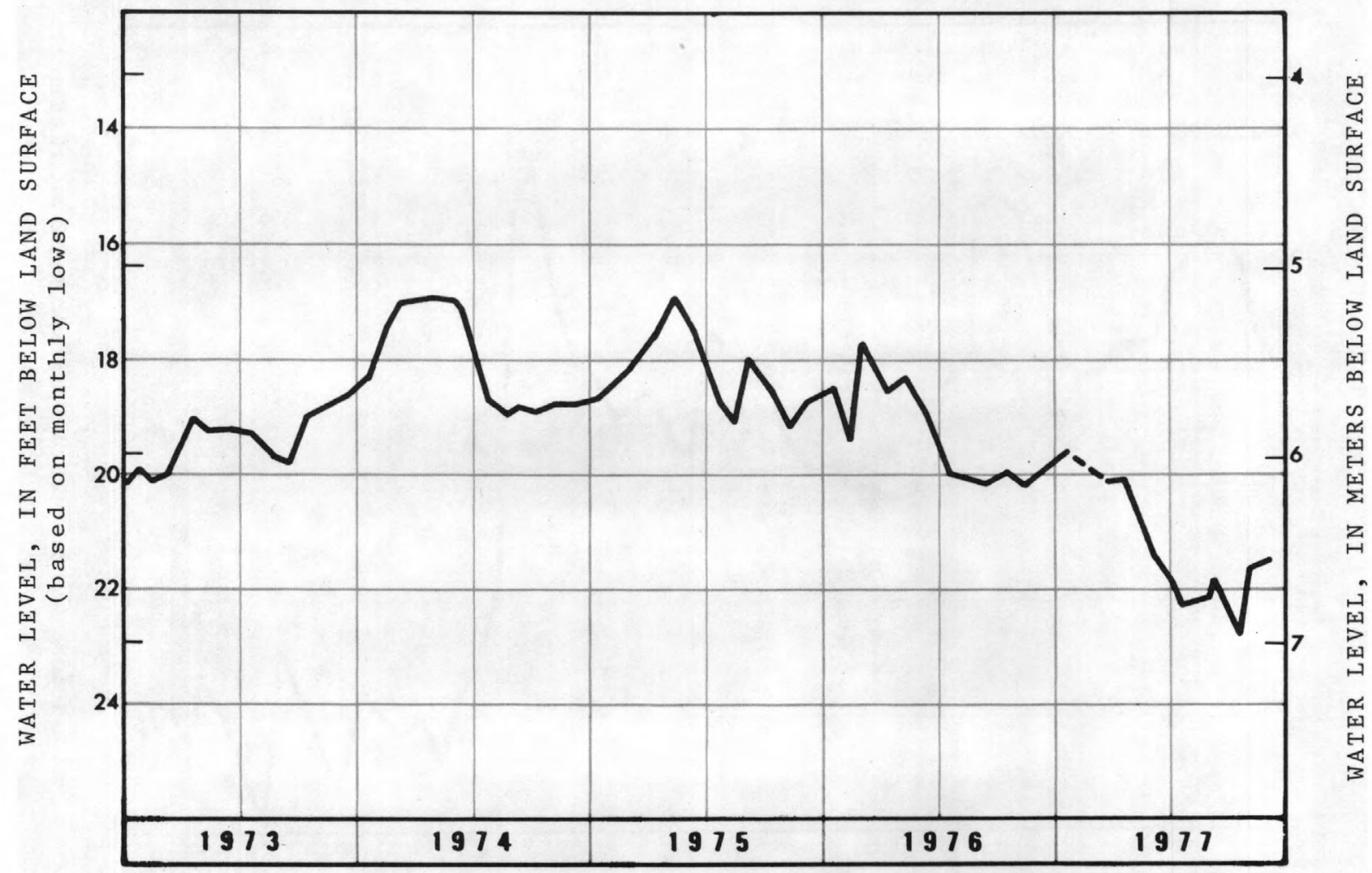

Water levels in observation we11 $1 \mathrm{~S} 7 \mathrm{~W}$ 32BD at Battle Creek. We11 is 95 feet deep and in the Marshall Formation. 
SUPPLY AND SOURCE -- 7 wells, about 500 feet deep, tap sandstones of the Saginaw Formation.

YIELD OF WELLS -- 250 to $500 \mathrm{ga} / \mathrm{min}$; specific capacity - 3 to $12 \mathrm{ga} 1 / \mathrm{min} / \mathrm{ft}$ of drawdown.

PUMPAGE -- Total annual pumpage, in million gallons, for past 5 years.

$1977-607$

1976 - 562

1975 - 559

1974 - 576

1973 - 583

$\begin{array}{lr}\text { QUALITY OF WATER - - } & \text { Hardness } \\ & \text { Iron } \\ & \text { Diss. Solids } \begin{aligned} 260-310 \mathrm{mg} / \mathrm{L} \\ 0.15-1.0 \mathrm{mg} / \mathrm{L} \\ 360-390 \mathrm{mg} / \mathrm{L}\end{aligned}\end{array}$

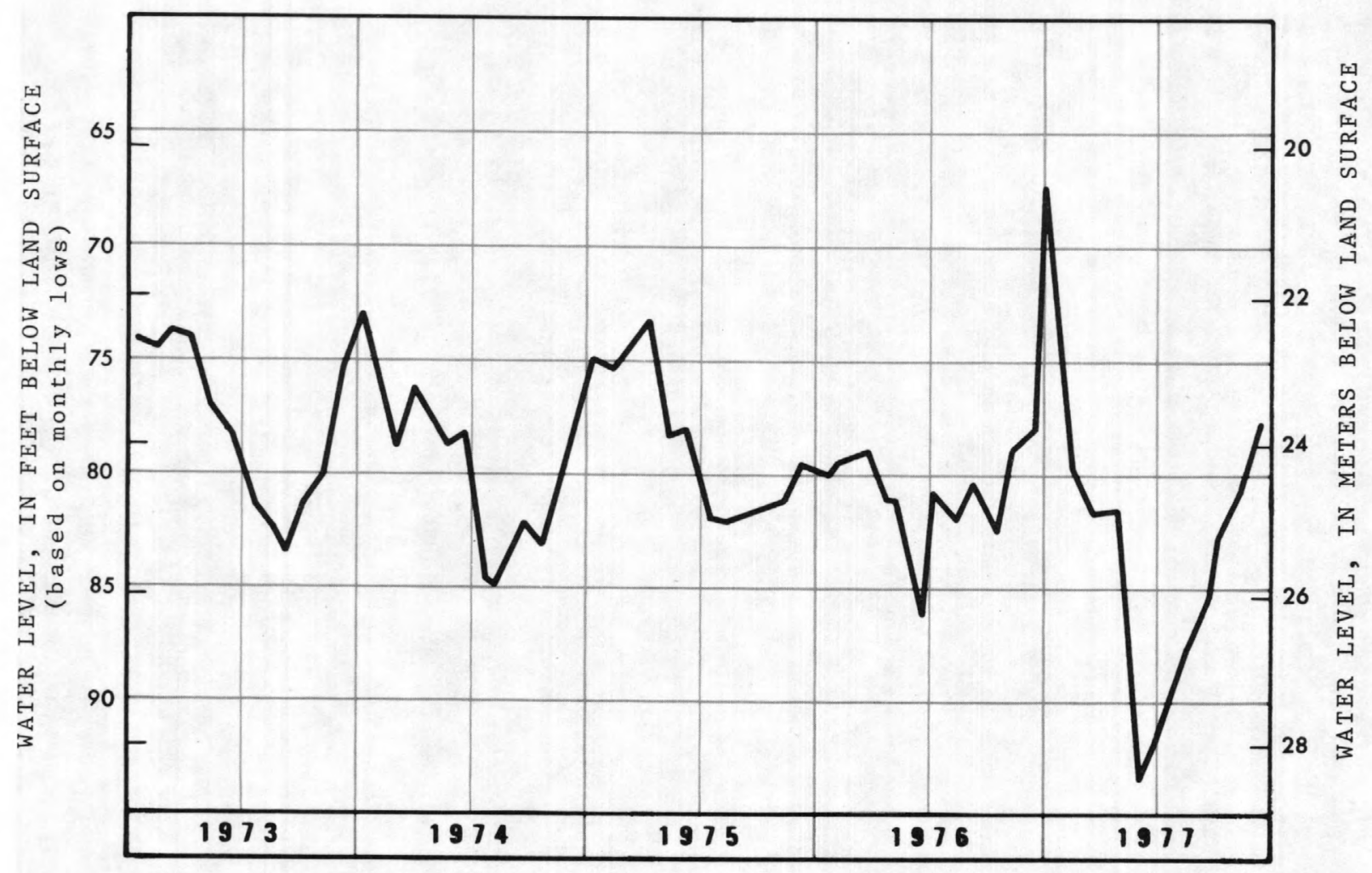

Water levels in observation well $7 \mathrm{~N} 2 \mathrm{~W} 9 \mathrm{BB}$ at St. Johns. We1l is 535 feet deep and in the Saginaw Formation. 
SUPPLY AND SOURCE -- 4 we11s, 370 to 450 feet deep, tap the Saginaw Formation. YIELD OF WELLS -- 160 to $700 \mathrm{ga} 1 / \mathrm{min}$.

PUMPAGE - Total annual pumpage, in million gallons, for past 5 years.

$$
\begin{aligned}
& 1977-645 \\
& 1976-625 \\
& 1975-541 \\
& 1974-520 \\
& 1973-435
\end{aligned}
$$

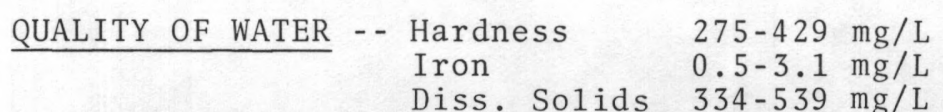


SUPPLY AND SOURCE -- 6 we11s, 136 to 223 feet deep, tap buried outwash deposits in glacial drift.

YIELD OF WELLS -- 350 to $500 \mathrm{gal} / \mathrm{min}$; specific capacity - 8 to $15 \mathrm{gal} / \mathrm{min} /$ ft of drawdown.

PUMPAGE -- Total annual pumpage, in million gallons, for past 5 years.

$$
\begin{aligned}
& 1977-533 \\
& 1976-542 \\
& 1975-500 \\
& 1974-530 \\
& 1973-547
\end{aligned}
$$

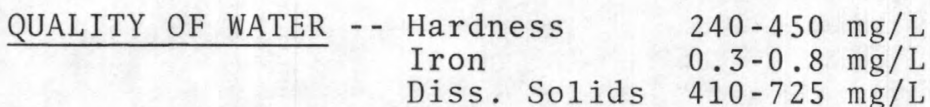

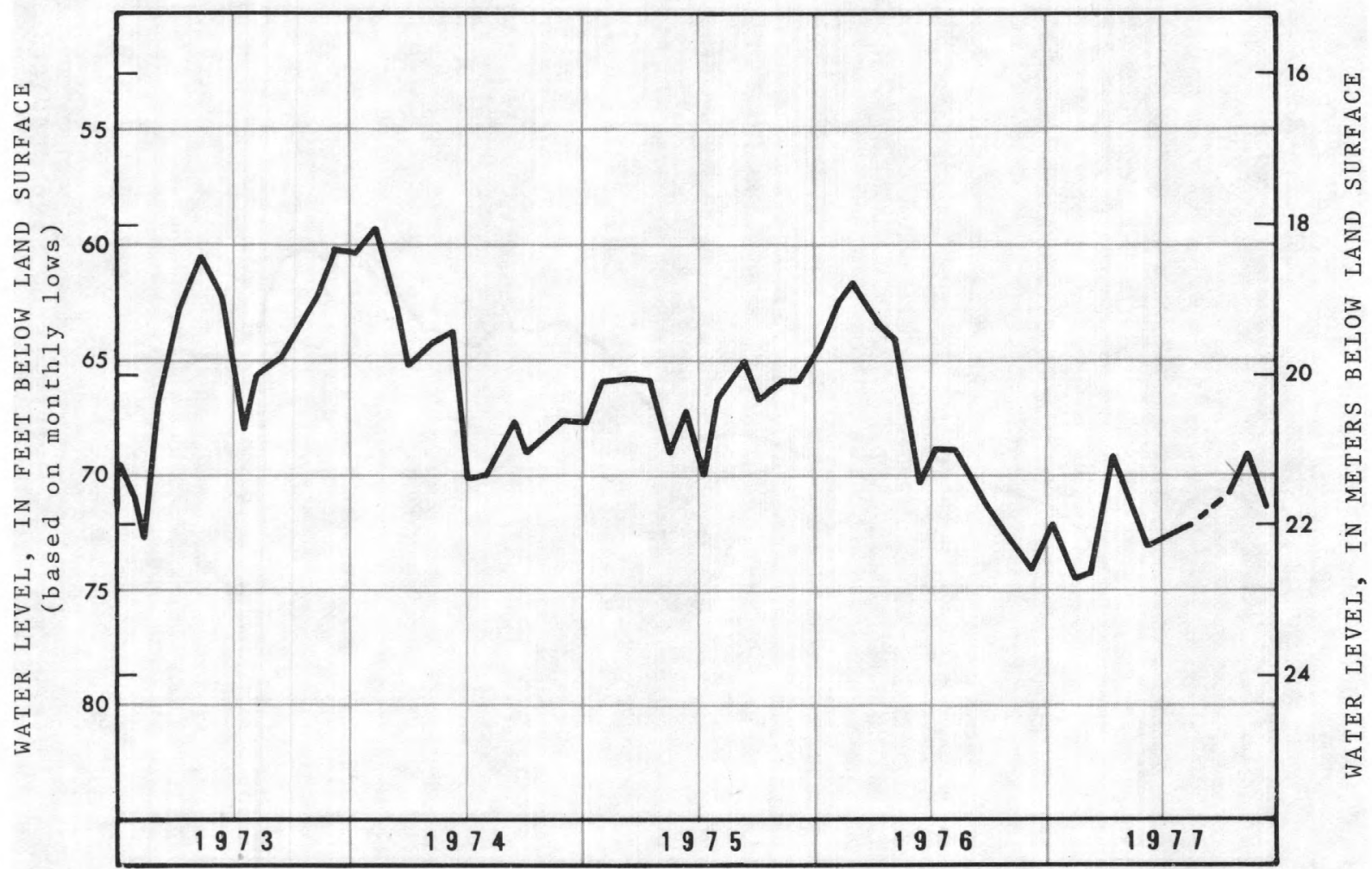

Water levels in observation well $12 \mathrm{~N} 3 \mathrm{~W} 24 \mathrm{DA}$ at St. Louis. We11 is 216 feet deep and in glacial deposits. 
SUPPLY AND SOURCE - - 125 we11s, 400 to 425 feet deep, tap sandstones of

the Saginaw Formation; 3 we11s, 85 to 105 feet deep, tap sand beds in glacial drift.

YIELD OF WELLS - - Sandstone - 100 to $700 \mathrm{gal} / \mathrm{min}$; specific capacity - 3 to $10 \mathrm{ga} 1 / \mathrm{min} / \mathrm{ft}$ of drawdown.

-- Glacial drift - 790 to 1,200 gal/min, specific capacity -

12 to $80 \mathrm{gal} / \mathrm{min} / \mathrm{ft}$ of drawdown.

PUMPAGE -- Total annual pumpage, in million gallons, for past 5 years.

$1977-9,203$

$1976-8,976$

$1975-8,099$

$1974-8,053$

$1973-8,850$

QUALITY OF WATER -- Composite at P1ant

$\begin{array}{lr}\text { Hardness } & 385 \mathrm{mg} / \mathrm{L} \\ \text { Iron } & 0.88 \mathrm{mg} / \mathrm{L} \\ \text { Diss. Solids } & 456 \mathrm{mg} / \mathrm{L}\end{array}$

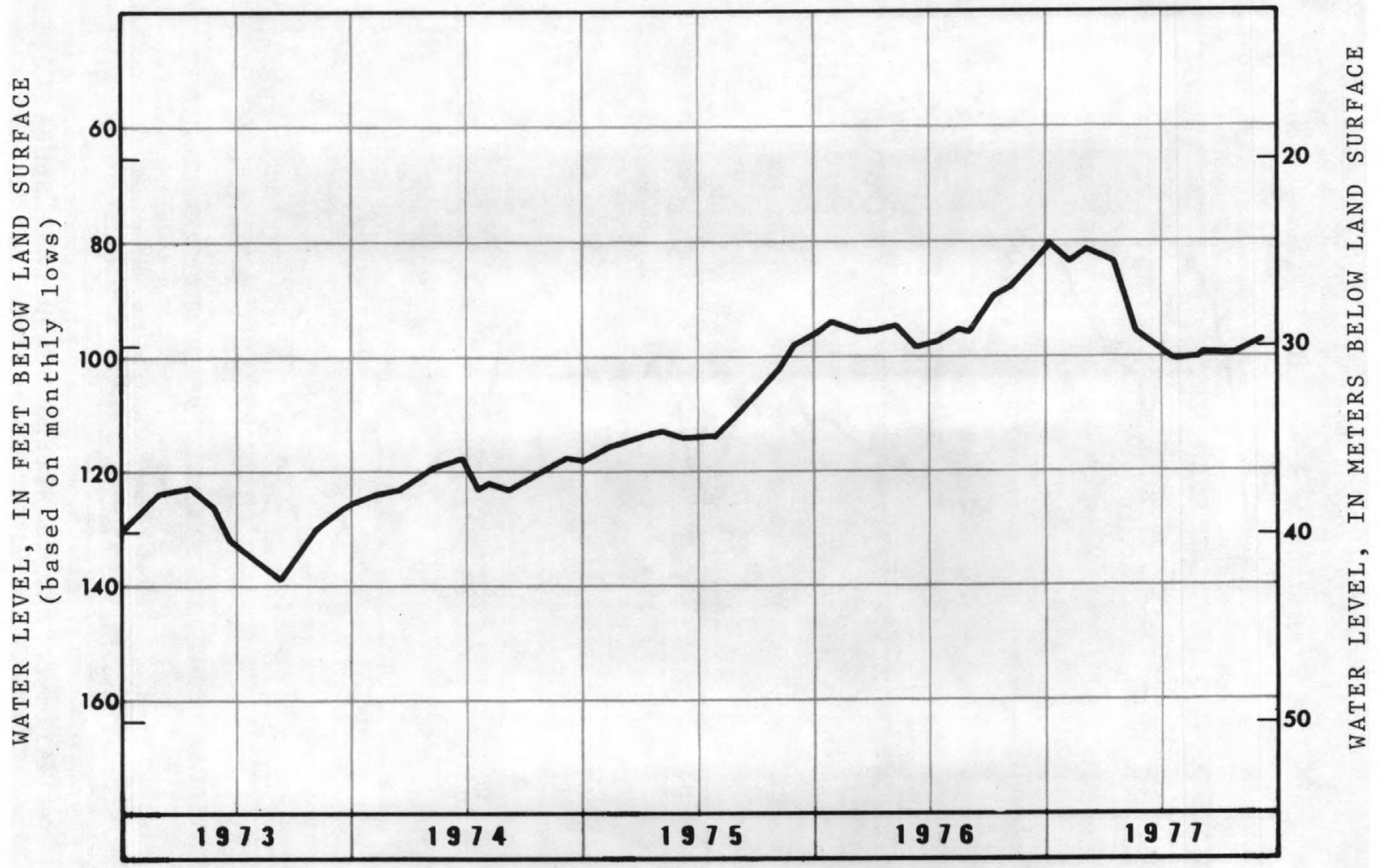

Water levels in observation well $4 \mathrm{~N} 2 \mathrm{~W} 9 \mathrm{BD}$ at Lansing. We11 is 401 feet deep and in the Saginaw Formation. 


\section{INGHAM COUNTY - CITY OF MASON}

SUPPLY AND SOURCE -- 1 we11, about 50 feet deep, taps the glacial drift; 1 we11, 223 feet deep, taps sandstones of the Saginaw Formation.

YIELD OF WELLS -- 675 to $700 \mathrm{ga} / \mathrm{min}$; specific capacity -- No. 3 yields $30 \mathrm{ga} / \mathrm{min} / \mathrm{ft}$ of drawdown from the glacial drift.

PUMPAGE - - Total annual pumpage, in million gallons, for past 5 years.

$$
\begin{aligned}
& 1977-206 \\
& 1976-218 \\
& 1975-211 \\
& 1974-222 \\
& 1973-179
\end{aligned}
$$
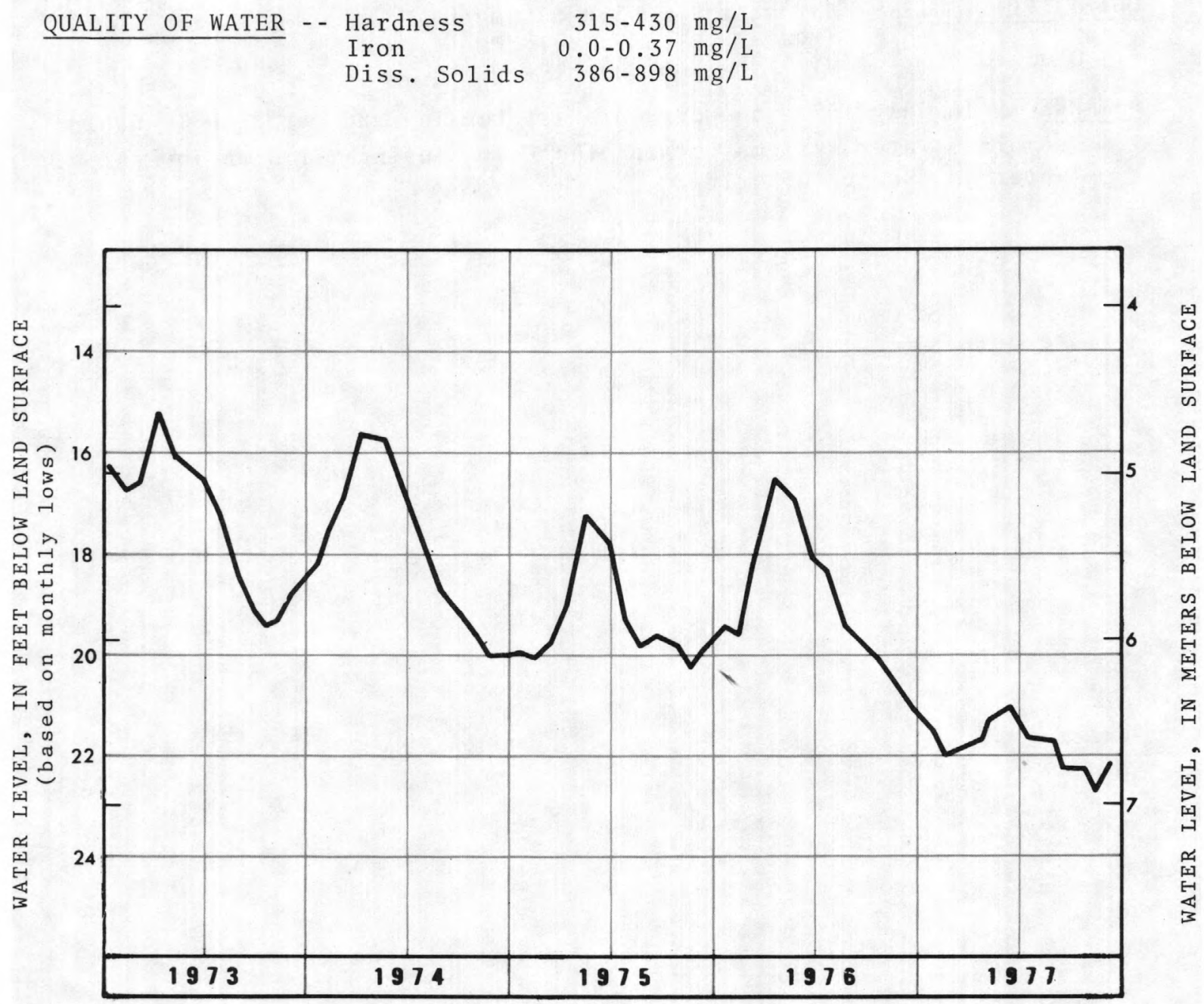

Water levels in observation well $2 \mathrm{~N} 1 \mathrm{~W}$ 5BB at Mason. We11 is 210 feet deep and in the Saginaw Formation. 
SUPPLY AND SOURCE -- 24 wells, 295 to 422 feet deep, tap the Saginaw Formation, and 1 well taps the glacial deposits.

YIELD OF WELLS -- About 280 to 1,000 gal/min; specific capacity 2 to 12 gal/min/ft of drawdown.

PUMPAGE -- Tota1 annual pumpage, in miliion gallons. $1977-1,654$

$1976-1,599$

$1975-1,566$

$1974-1,487$

\begin{tabular}{|c|c|c|}
\hline $0 F$ & $\begin{array}{l}\text { Iron } \\
\text { Diss. Solids }\end{array}$ & $\begin{array}{l}310-505 \\
0.9-3.8 \\
345-662\end{array}$ \\
\hline
\end{tabular}

REMARKS - - In Ju1y 1973, the city of East Lansing and Meridian Township combined water systems forming the East Lansing-Meridian Township Water and Sewer Authority. 
SUPPLY AND SOURCE -- 7 we11s, 399 to 417 feet deep, tap sandstones of the Saginaw Formation.

YIELD OF WELLS - - 260 to $500 \mathrm{gal} / \mathrm{min}$; specific capacity - 3 to $8 \mathrm{gal} / \mathrm{min} / \mathrm{ft}$ of drawdown.

PUMPAGE -- Total annual pumpage, in million gallons, for past 5 years.

$$
\begin{aligned}
& 1977-508 \\
& 1976-586 \\
& 1975-725 \\
& 1974-631 \\
& 1973-750
\end{aligned}
$$
QUALITY OF WATER -- Hardness 274-435 mg/L Iron $\quad 0.35-13.0 \mathrm{mg} / \mathrm{L}$ Diss. Solids $320-528 \mathrm{mg} / \mathrm{L}$

REMARKS -- Most ground-water pumped by the township is used to supply industrial plants in the area. 
SUPPLY AND SOURCE -- 17 we11s, 353 to 435 feet deep, tap sandstones of the Saginaw Formation; 2 wells are on a standby basis only.

YIELD OF WELLS -- 147 to $654 \mathrm{gal} / \mathrm{min}$; specific capacity -- 1 to $11 \mathrm{ga} 1 / \mathrm{min} / \mathrm{ft}$ of drawdown.

PUMPAGE -- Tota1 annual pumpage, in million gallons, for past 5 years.

$$
\begin{aligned}
& 1977-1,675 \\
& 1976-1,731 \\
& 1975-1,800 \\
& 1974-1,800 \\
& 1973-1,805
\end{aligned}
$$

$\begin{array}{lr}\text { QUALITY OF WATER - - } & \text { Hardness } \\ \text { Iron } & \begin{aligned} & 315-350 \mathrm{mg} / \mathrm{L} \\ & \text { Diss. Solids } 0.15-1.20 \mathrm{mg} / \mathrm{L} \\ & 361-405 \mathrm{mg} / \mathrm{L}\end{aligned}\end{array}$




\section{JACKSON COUNTY - CITY OF JACKSON}

SUPPLY AND SOURCE -- 14 we11s, 380 to 400 feet deep, tap sandstones of the Saginaw and Marshal1 Formations.

YIELD OF WELLS -- 1,000 to $2,800 \mathrm{ga} 1 / \mathrm{min}$; specific capacity - - No. 12 wel1 is 56 ; reported average of all wells is $100 \mathrm{gal} / \mathrm{min} / \mathrm{ft}$ of drawdown.

PUMPAGE -- Total annual pumpage, in million gallons, for past 5 years.

$$
\begin{aligned}
& 1977-4,330 \\
& 1976-4,104 \\
& 1975-4,077 \\
& 1974-4,634 \\
& 1973-4,864
\end{aligned}
$$

\begin{tabular}{|c|c|c|}
\hline $\mathrm{F}$ & $\begin{array}{l}\text {-- Hardness } \\
\text { Iron } \\
\text { Diss. Solids }\end{array}$ & $\begin{array}{l}260-630 \\
0.2-1.0 \\
394-1072\end{array}$ \\
\hline
\end{tabular}

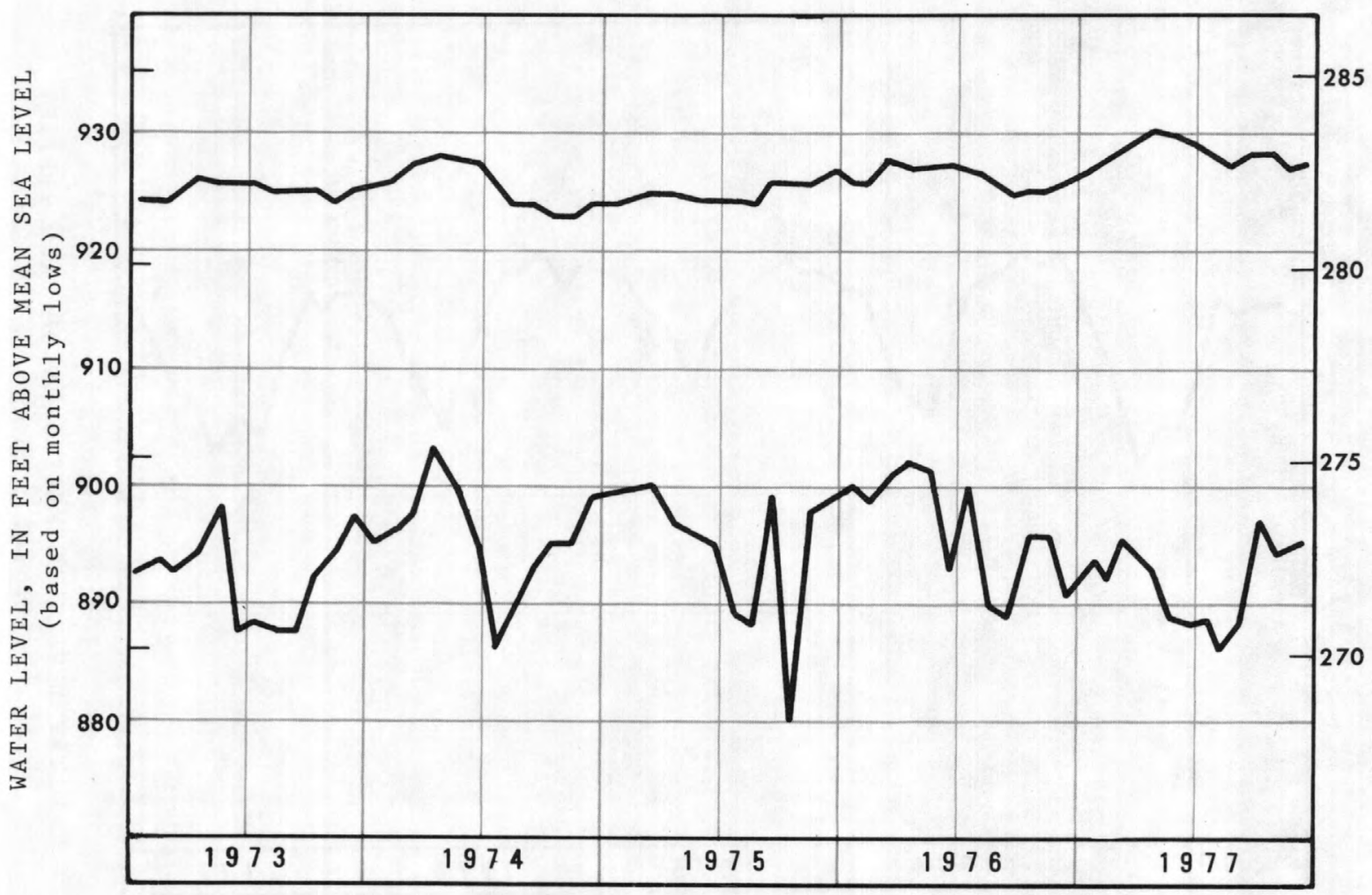

Water leve1s in observation we11s 3S 1W $11 \mathrm{AA} 2$ (top), and 3S 1W 2BD (bottom). We11 3S 1W 11AA2 is 36 feet deep and in glacial deposits. We11 3S $1 W 2 \mathrm{BD}$ is 400 feet deep and in the Saginaw and Marshall Formations. 


\section{KALAMAZOO COUNTY - CITY OF KALAMAZOO}

SUPPLY AND SOURCE -- 84 we11s, 130 to 254 feet deep, tap the glacial drift.

YIELD OF WELLS - 200 to $2,000 \mathrm{gal} / \mathrm{min}$; specific capacity - 7 to 100 $\mathrm{gal} / \mathrm{min} / \mathrm{ft}$ of drawdown.

PUMPAGE -- Total annual pumpage, in million gallons, for past 5 years.

$$
\begin{aligned}
& 1977-6,476 \\
& 1976-6,549 \\
& 1975-6,336 \\
& 1974-6,454 \\
& 1973-6,689
\end{aligned}
$$

QUALITY OF WATER -- Composite of 2 pumping stations:

$$
\begin{array}{lc}
\text { Hardness } & 385-420 \mathrm{mg} / \mathrm{L} \\
\text { Iron } & 0.49-3.10 \mathrm{mg} / \mathrm{L} \\
\text { Diss. Solids } & 499-530 \mathrm{mg} / \mathrm{L}
\end{array}
$$

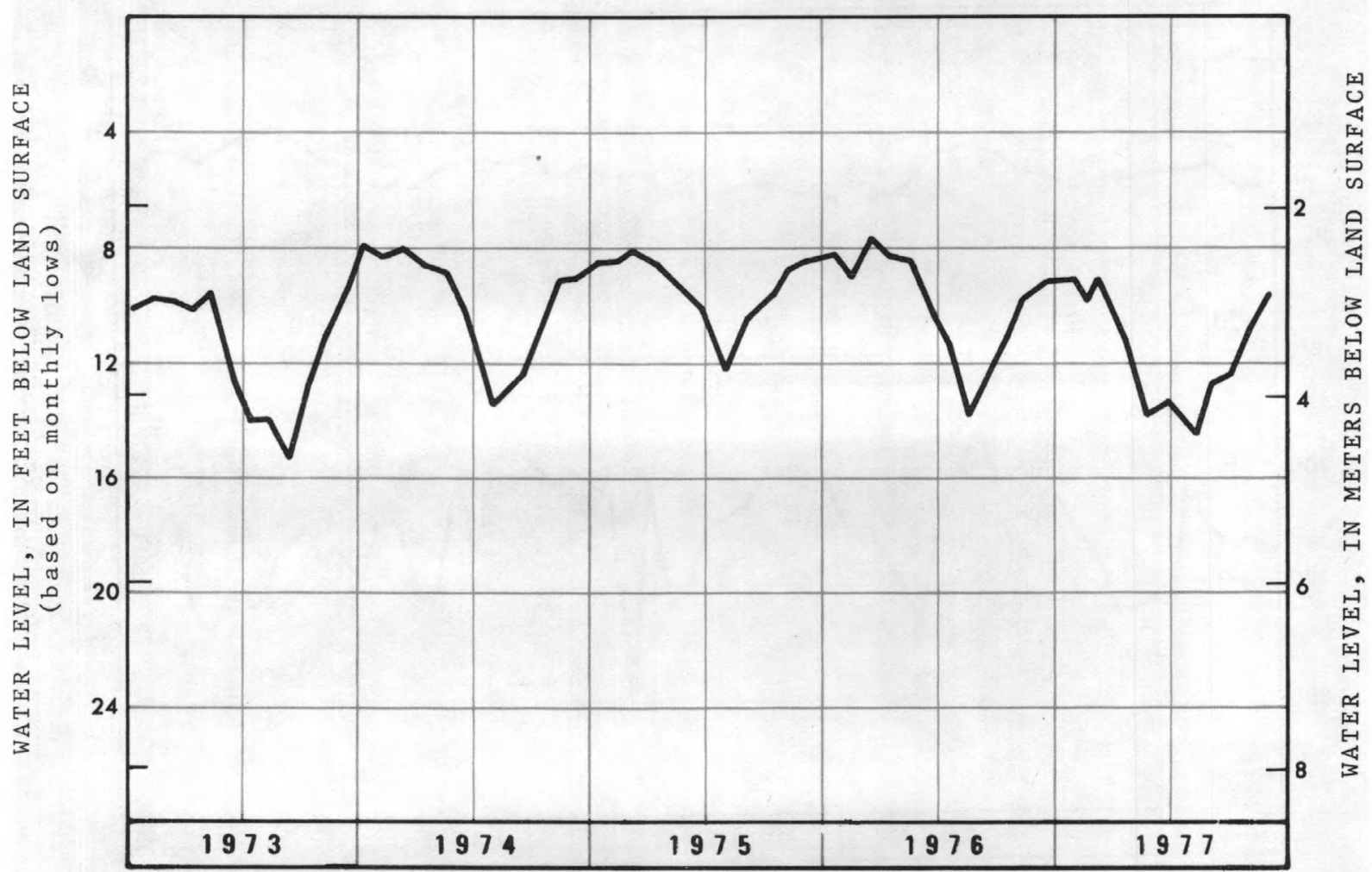

Water levels in observation well 2S $11 \mathrm{~W} 22 \mathrm{CD}$ at Kalamazoo. Well is $137 \mathrm{feet}$ deep and in glacial deposits. 
SUPPLY AND SOURCE - - 15 we11s, 95 to 185 feet deep, tap the glacial drift.

YIELD OF WELLS - 300 to $1,000 \mathrm{ga} 1 / \mathrm{min}$; specific capacity - $25 \mathrm{ga} 1 / \mathrm{min} / \mathrm{ft}$ of drawdown.

PUMPAGE -- Total annual pumpage, in million gallons, for past 5 years.

$$
\begin{aligned}
& 1977-802 \\
& 1976-784 \\
& 1975-631 \\
& 1974-647 \\
& 1973-582
\end{aligned}
$$

$$
\begin{aligned}
& \text { QUALITY OF WATER -- Hardness 165-345 mg/L } \\
& \text { Iron } \quad 0.0-1.5 \mathrm{mg} / \mathrm{L} \\
& \text { Diss. Solids 200-469 mg/L }
\end{aligned}
$$

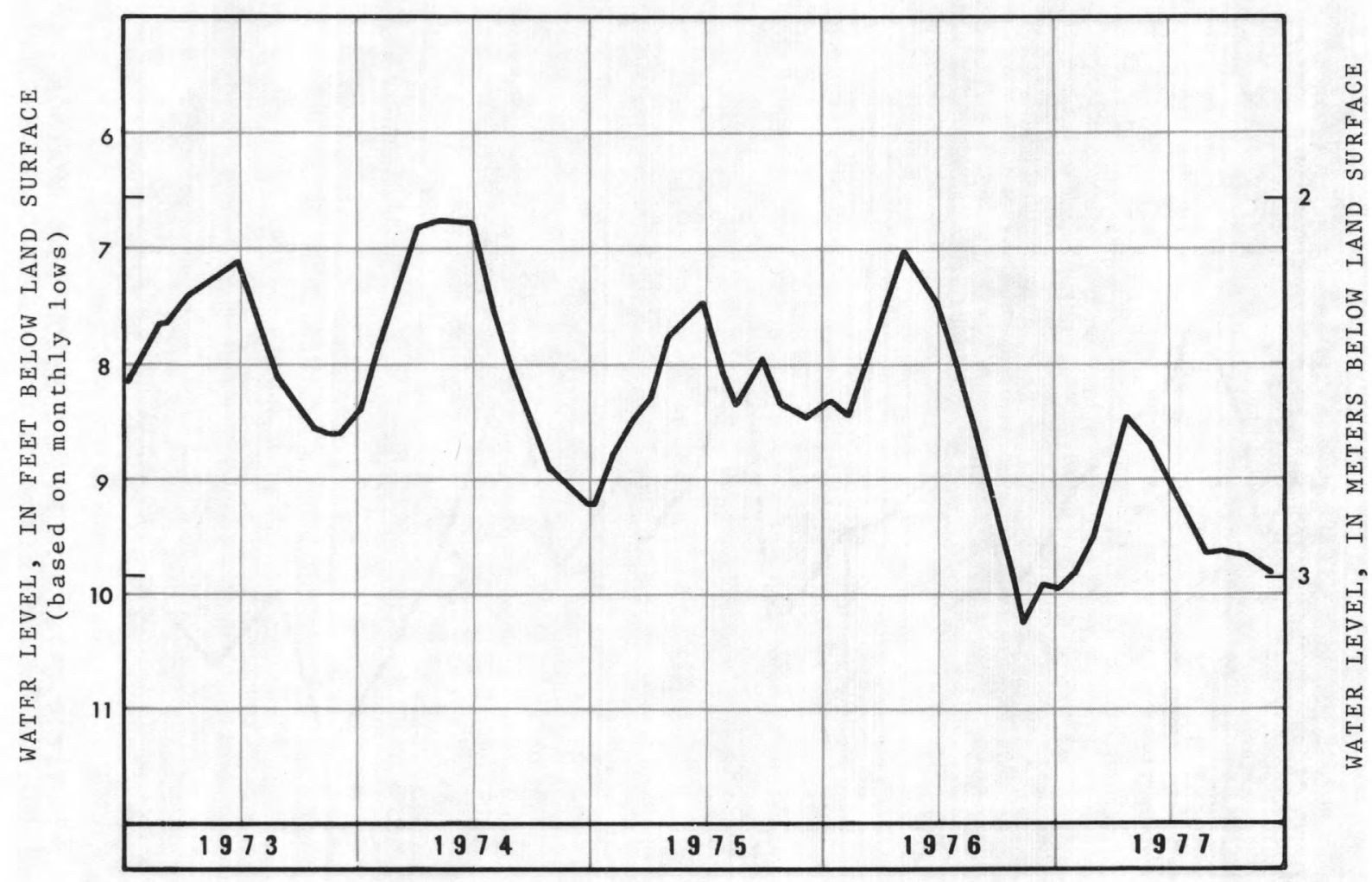

Water levels in observation well 3S $11 \mathrm{~W} 22 \mathrm{BD}$ at Portage. Well is 120 feet deep and in glacial deposits. 

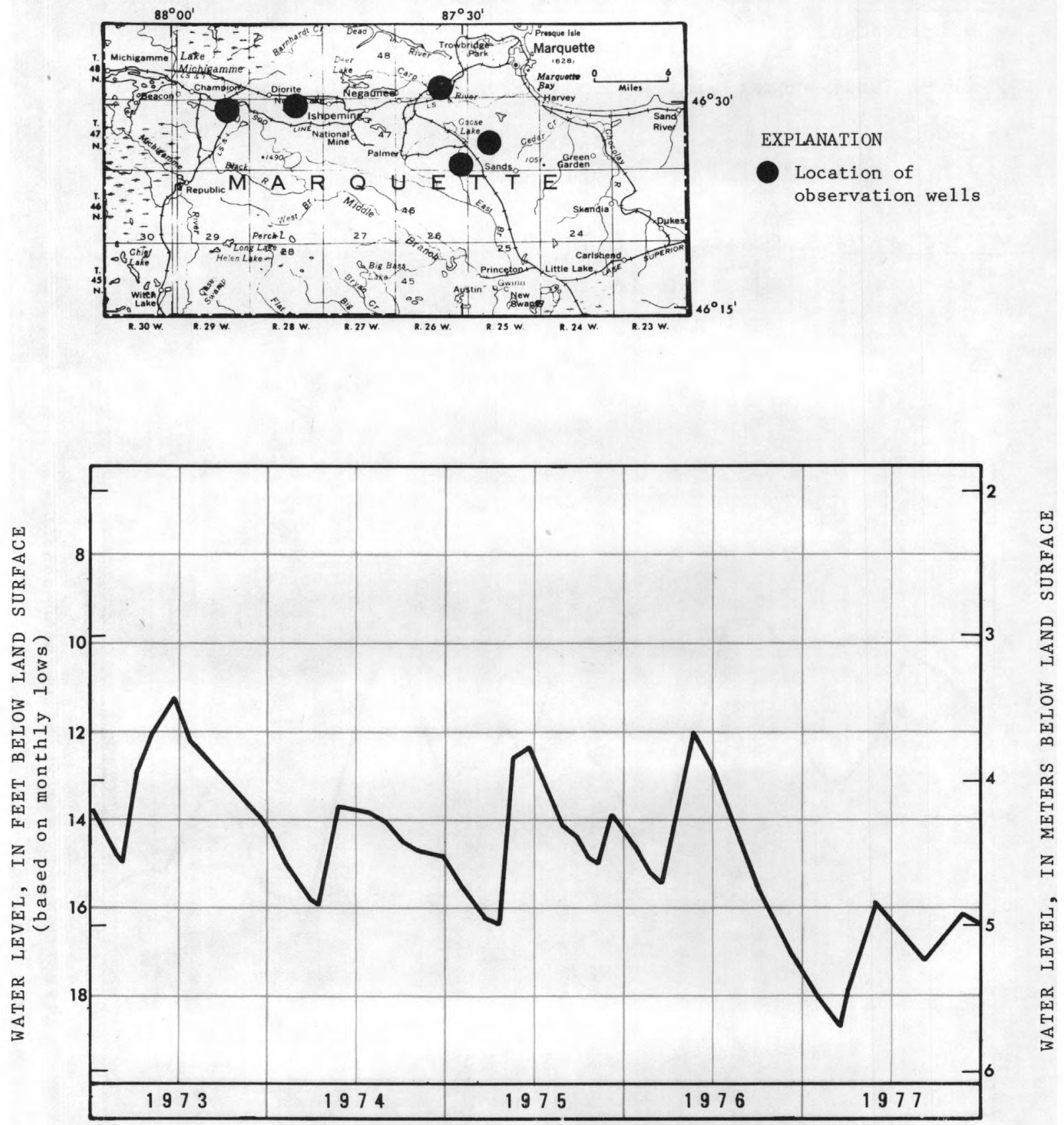

Water 1 evels in observation we11 47N 28W 3CC in Marquette County. Well is 75 feet deep and in glacial deposits. Levels shown are typical of observation wells located in the Marquette Iron Range. 


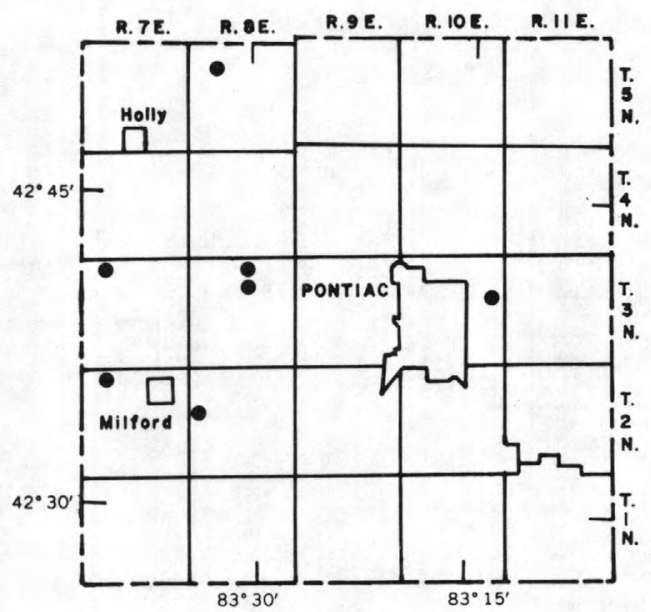

EXPLANATION

Location of
observation wells

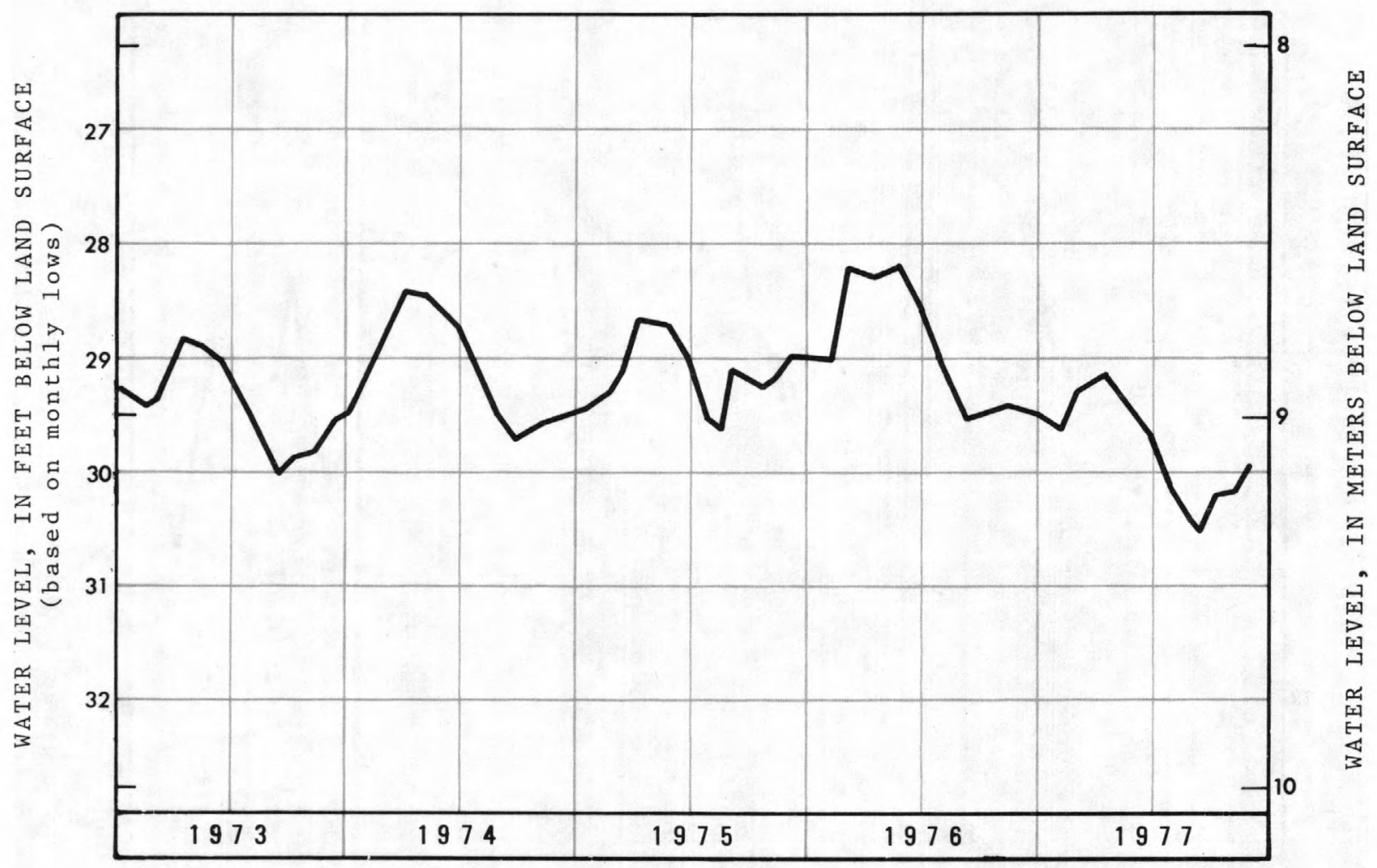

Water levels in observation we11 3N 8E 10AB. We11 is 163 feet deep and in glacial deposits. 


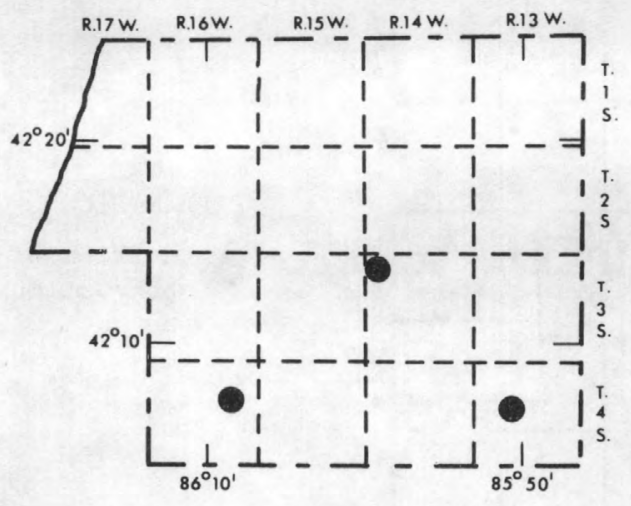

EXPLANATION

Location of

observation we1ls

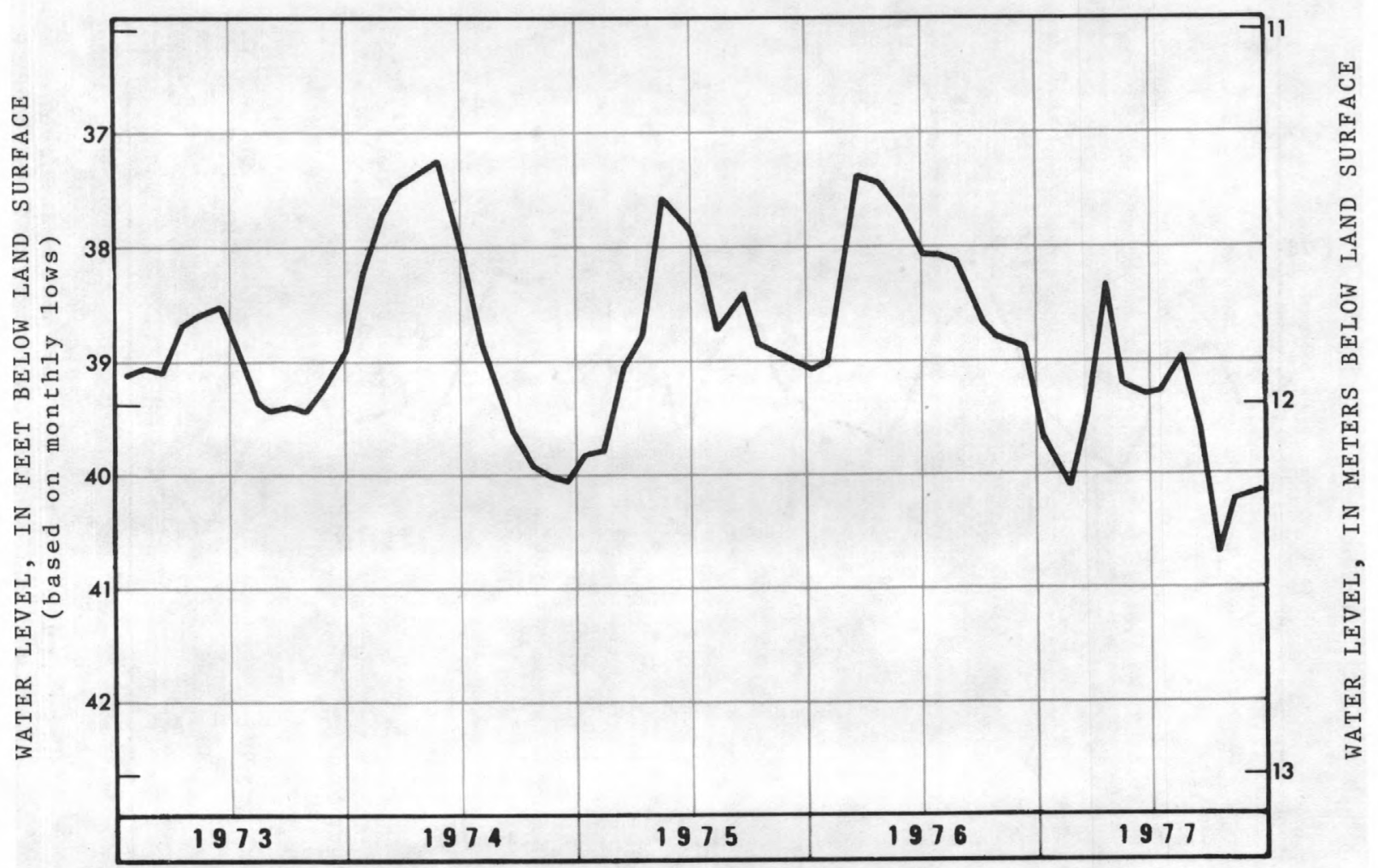

Water levels in observation we11 $3 \mathrm{~S} 14 \mathrm{~W} 6 \mathrm{BA}$ in Van Buren County. We11 is 59 feet deep and in glacial deposits. Levels are typical of other observation wells located in the county. 
SUPPLY AND SOURCE -- 3 wells, 91 to 196 feet deep, tap the glacial drift; most water is pumped from the Huron River.

YIELD OF WELLS -- 1,050 to $4,860 \mathrm{gal} / \mathrm{min}$; specific capacity - 20 to 600 $\mathrm{ga} 1 / \mathrm{min} / \mathrm{ft}$ of drawdown.

PUMPAGE -- Tota1 annual ground-water pumpage, in million gallons, for past 5 years (only 15 to 20 percent of total pumpage is from ground-water sources).

$$
\begin{aligned}
& 1977-674 \\
& 1976-677 \\
& 1975-878 \\
& 1974-958 \\
& 1973-899
\end{aligned}
$$

QUALITY OF WATER -- Ground water:

$$
\begin{array}{lr}
\text { Hardness } & 355-585 \mathrm{mg} / \mathrm{L} \\
\text { Iron } & 0.25-2.4 \mathrm{mg} / \mathrm{L}
\end{array}
$$

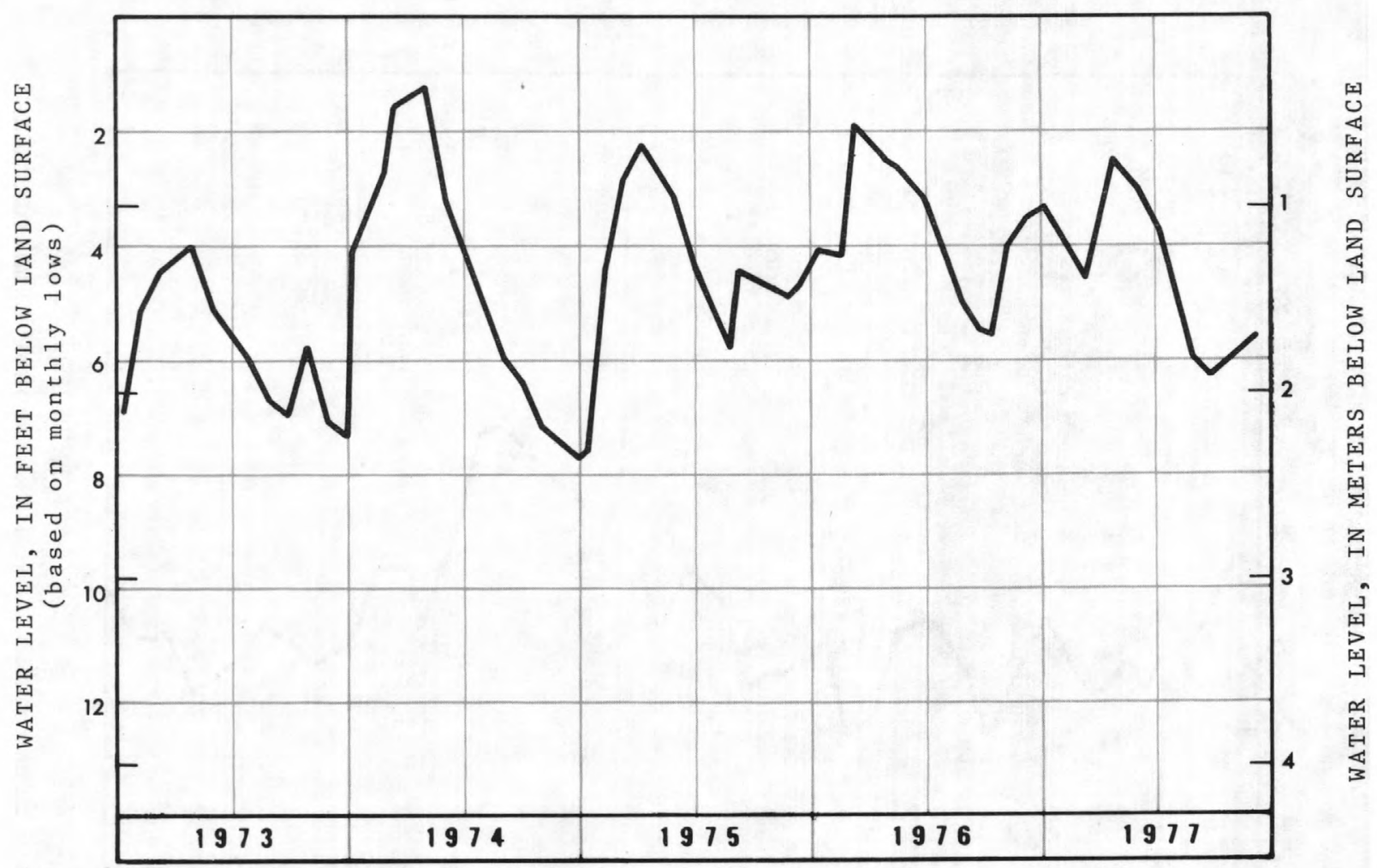

Water leve1s in observation we11 3S 6E 16BC at Ann Arbor. Well is 55 feet deep and in glacial deposits. 
SUPPLY AND SOURCE -- 7 wells, 87 to 102 feet deep, tap the glacial drift.

YIELD OF WELLS -- Average 450 gal/min; specific capacity - - 25 to 180 $\mathrm{gal} / \mathrm{min} / \mathrm{ft}$ of drawdown.

PUMPAGE -- Total annual pumpage, in million gallons, for past 5 years.

$$
\begin{aligned}
& 1977-1,440 \\
& 1976-1,652 \\
& 1975-1,462 \\
& 1974-1,958 \\
& 1973-1,920
\end{aligned}
$$

$$
\begin{aligned}
& \text { QUALITY OF WATER -- Hardness 305-320 mg/L } \\
& \text { Iron } 1.2-5.0 \mathrm{mg} / \mathrm{L} \\
& \text { Diss. Solids 400-764 mg/L }
\end{aligned}
$$

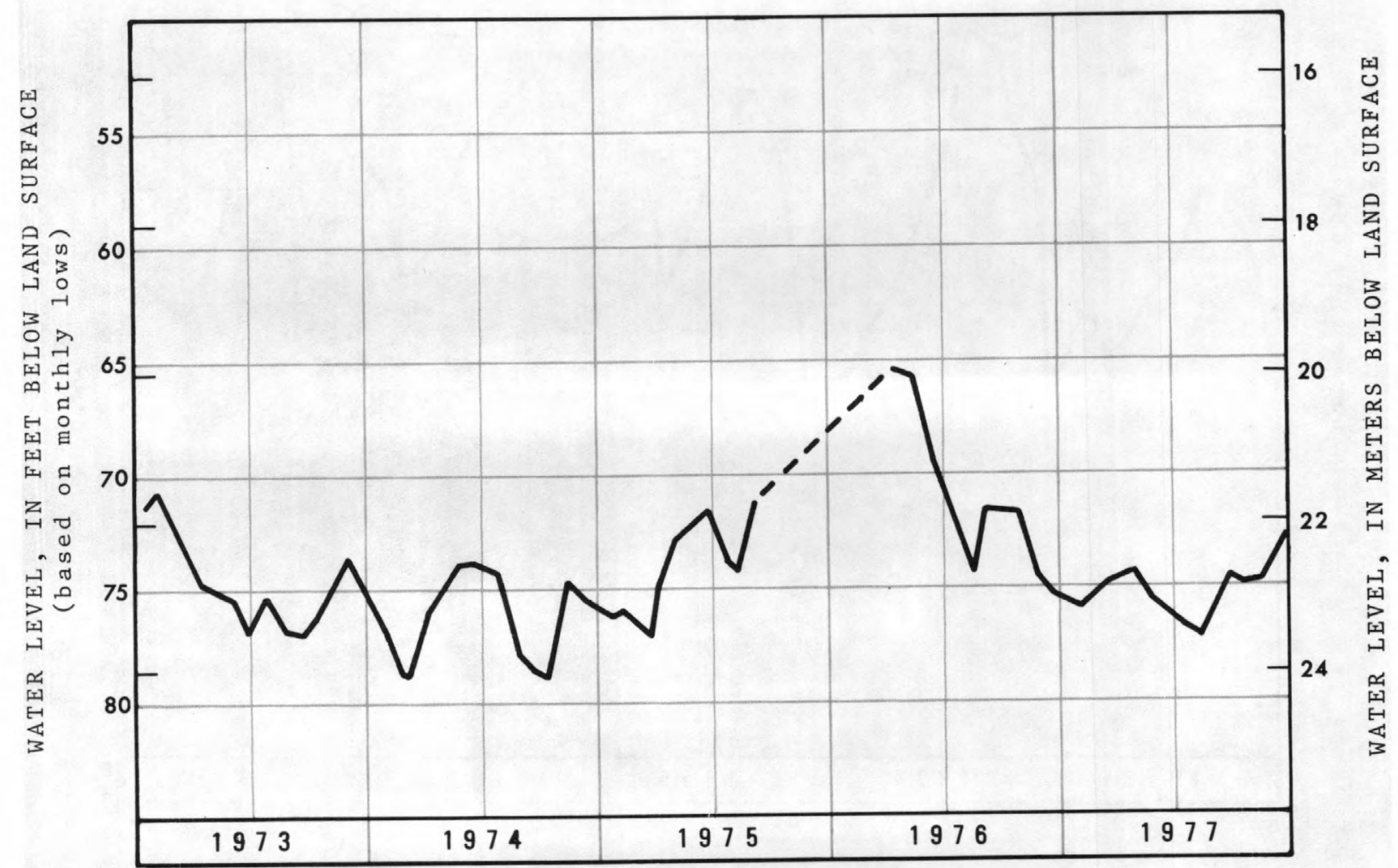

Water levels in observation well 3S 7E 9AD at Ypsilanti. Well is 94 feet deep and in glacial deposits. 
SUPPLY AND SOURCE -- 8 wells, 50 to 95 feet deep, tap the glacial drift.

YIELD OF WELLS -- 700 to $3,500 \mathrm{gal} / \mathrm{min}$.

PUMPAGE - Total annual pumpage, in million gallons, for past 5 years.

$$
\begin{array}{r}
1977-792 \\
1976-1,067 \\
1975-854 \\
1974-1,715 \\
1973-2,284
\end{array}
$$

QUALITY OF WATER -- Composite

$$
\begin{array}{ll}
\text { Hardness } & 370 \mathrm{mg} / \mathrm{L} \\
\text { Iron } & 0.5 \mathrm{mg} / \mathrm{L} \\
\text { Diss. Solids } & 496 \mathrm{mg} / \mathrm{L}
\end{array}
$$

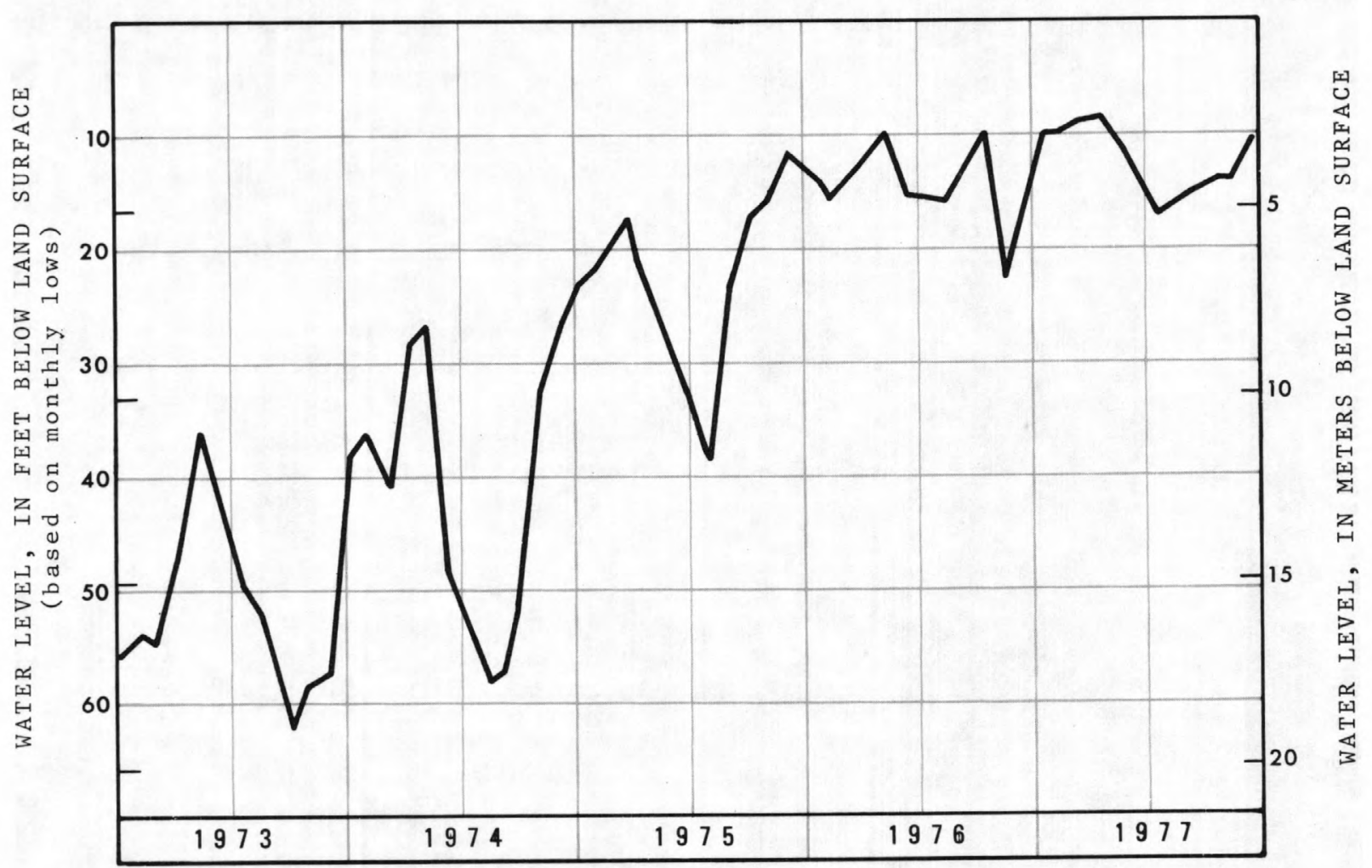

Water levels in observation we11 3S 7E 24CD in Ypsilanti Township. Well is 75 feet deep and in glacial deposits. 
TABLE 1. RECORDS OF MICHIGAN OBSERVATION WELLS.

COUNTY AND WELL NUMBER: For explanation of well numbers, see text under - Introduction 'We11 numbering system":

NAME: MDNR - Michigan Department of Natural Resources; WMP - Wisconsin-Michigan Power Company; MSHD - Michigan State Highway

Department; USFS - U.S. Forest Service; HCMA - Huron-Clinton Metropolitan Authority; BCRC - Branch County Road Conmission. AQUIFER:

Qgd - Glacial drift deposits of Pleistocene (Quaternary) age

Ps - Saginaw Formation of Pennsylvanian age

Mm - Marsha1l Formation of Mississippian age

Dt - Traverse Croup of Middle and Late Devonian age

Ss - Salina Formation of Late Silurian age

$\mathrm{Sm}$ - Manistique Dolomite of Middle Silurian age

Or - Limestones of Richmondian age (Late Ordovician)

ALTITUDE: Land-surface datum in feet above mean sea leve1.

MEASUREMENT, 1977 (frequency) : R - Cont inuous recorder; D - Daily; W - Weekly; M - Monthly; Q - Quarterly; S - Semiannually;

A - Annually; I - Intermittent.

OBSERVED WATER-LEVEL EXTREMES: In feet below or above (+) 1and surface, 1977 measurements underscored are extremes for entire

REMARKS: P - Water levels affected by pumping. Water-level measurements are made by the U.S. Geological Survey unless otherwise noted.

\begin{tabular}{|c|c|c|c|c|c|c|c|c|c|c|c|c|}
\hline \multirow{3}{*}{$\begin{array}{c}\text { COUNTY } \\
\text { AND } \\
\text { WELL NUMBER } \\
\text { TWP., RANGE. SECTION }\end{array}$} & \multirow{3}{*}{ NAME } & \multirow{3}{*}{ 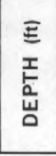 } & \multirow{3}{*}{ 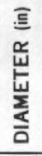 } & \multirow{3}{*}{ 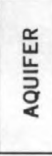 } & \multirow{3}{*}{ 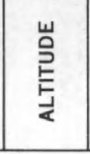 } & \multirow{3}{*}{ 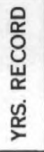 } & \multirow{3}{*}{$\frac{\hat{\sigma}}{\frac{\hat{\sigma}}{\dot{\omega}}}$} & \multicolumn{4}{|c|}{ OBSERVED WATER-LEVEL EXTREMES } & \multirow{3}{*}{ REMARKS } \\
\hline & & & & & & & & \multicolumn{2}{|c|}{ THROUGH 1976} & \multicolumn{2}{|c|}{ IN 1977} & \\
\hline & & & & & & & & MAXIMUM & MINIMUM & MAXIMUM & MINIMUM & \\
\hline \multicolumn{13}{|l|}{ ALGER } \\
\hline $45 N$ 19W $25 B D$ & $\mathrm{CCC}$ & 66 & 6 & Qgd & 850 & 19 & Q & 6.4 Jun 1960 & 14.2 Apr 1964 & 11.0 Apr & 12.3 Aug & \\
\hline \multicolumn{13}{|l|}{ ALPENA } \\
\hline $32 \mathrm{~N} 6 \mathrm{E} 23 \mathrm{DDDA1}$ & Alpena State Forest & 88 & 6 & Qgd & 713 & 1 & $\mathrm{R}$ & & & 21.0 May & $28.7 \mathrm{Mar}$ & \\
\hline \multicolumn{13}{|l|}{ BARAGA } \\
\hline $48 \mathrm{~N} 32 \mathrm{~W} 12 \mathrm{DD}$ & WMP 14 & 10 & 1 & Qgd & 1,630 & 30 & M & 3.3 Apr 1965 & 8.1 Sep 1969 & 5.7 Apr & $7.1 \mathrm{Feb}$ & Meas. by WMP \\
\hline \multicolumn{13}{|l|}{$\underline{\text { BARRY }}$} \\
\hline $4 \mathrm{~N}$ 9W $5 \mathrm{DA}$ & Solomon Road & 131 & 2 & Qgd & 860 & 14 & Q & $113.2 \mathrm{Dec} 1976$ & $122.0 \mathrm{Mar} 1965$ & $\underline{112.7 \mathrm{Feb}}$ & 116.1 May & \\
\hline \multicolumn{13}{|l|}{$\underline{B A Y}$} \\
\hline 17N 4E 22DCAAl & Pinconning Township & 110 & 6 & Ps & 620 & 16 & $\mathrm{R}$ & 0.0 Mar 1976 & 10.5 Aug 1963 & $1.1 \mathrm{Dec}$ & 5.7 May & \\
\hline \multicolumn{13}{|l|}{$\underline{\text { BRANCH }}$} \\
\hline $5 S 6 \mathrm{~W} 22 \mathrm{BB}$ & Girard & 27 & 1 & Qgd & 950 & 14 & M & 10.5 Mar 1974 & 16.3 Nov 1964 & $13.2 \mathrm{Apr}$ & $14.9 \mathrm{Jan}$ & \\
\hline $8 \mathrm{~W} 28 \mathrm{DB}$ & Sherwood & 42 & 1 & Qgd. & 880 & 13 & M & 11.3 Mar 1976 & 18.9 Nov 1965 & 16.7 Apr & $18.8 \mathrm{Jan}$ & \\
\hline $6 \mathrm{~S} 6 \mathrm{~W} 18 \mathrm{CCCD} 1$ & Coldwater Township & 56 & 6 & Qgd & 950 & 14 & M & 18.3 Mar 1976 & 28.3 Jul 1964 & $23.3 \mathrm{Jan}$ & 26.3 Sep & \\
\hline $22 \mathrm{CA}$ & Coldwater Test 4 & 113 & 6 & Qgd & 970 & 14 & $\mathrm{R}$ & 9.0 May 1975 & 24.8 Aug 1976 & 11.9 Apr & 25.9 May & $\mathrm{P}$ \\
\hline $855 W 6 A B$ & Chipman & 55 & 4 & Qgd & 1,032 & 14 & M & 13.9 Feb 1968 & 19.4 Dec 1964 & 16.4 May & $18.5 \mathrm{Mar}$ & \\
\hline 8W $17 \mathrm{CD}$ & Bronson School & 38 & 1 & Qgd & 917 & 14 & M & 13.1 May 1966 & 16.3 Nov 1964 & $14.9 \mathrm{Apr}$ & $15.8 \mathrm{Jan}$ & \\
\hline \multicolumn{13}{|l|}{ CALHOUN } \\
\hline 1S $7 \mathrm{~W} 10 \mathrm{BB}$ & Sabin & 12 & 15 & Qgd & 908.0 & 32 & W & $0.9 \operatorname{Mar} 1950$ & 7.2 Dec 1964 & $3.8 \mathrm{Apr}$ & $5.1 \mathrm{Feb}$ & Meas. by owner \\
\hline $32 \mathrm{BDCC} 1$ & Penfield Township & 95 & 6 & Mn & 845 & 14 & $\mathrm{R}$ & 15.6 Apr 1974 & 27.0 Aug 1964 & $18.1 \mathrm{Apr}$ & 22.8 oct & $P$ \\
\hline $32 \mathrm{DA}$ & Battle Creek & 127 & 8 & $\mathrm{Mm}$ & 830.8 & 39 & $\mathrm{D}$ & 0.7 Apr 1950 & 16.8 Jul 1959 & 4.9 Jan & $9.8 \mathrm{Jul}$ & P, Meas. by owner \\
\hline $2 \mathrm{~S} 6 \mathrm{~W} 25 \mathrm{AA}$ & Marsha11 & 59 & 6 & $\mathrm{Mm}$ & 904.8 & 28 & M & 5.5 May 1950 & 9.7 Aug 1964 & $7.8 \mathrm{Jan}$ & 9.2 Sep & $P$, Meas. by owner \\
\hline \multicolumn{13}{|l|}{ CASS } \\
\hline $8 \mathrm{~S} 14 \mathrm{~W} 17 \mathrm{BA}$ & Little & 55 & 28 & Qgd & 840 & 33 & M & 46.2 Jul 1950 & 55.0 Mar 1957 & $50.7 \mathrm{Jan}$ & 51.8 Oct & \\
\hline \multicolumn{13}{|l|}{ CHEBOYGAN } \\
\hline $33 \mathrm{~N} 1 \mathrm{1W} 26 \mathrm{DABB} 1$ & Pigeon River CCC & 164 & 6 & Qgd & 933 & 12 & $\mathrm{R}$ & 56.2 May 1971 & 59.9 Dec 1965 & 58.4 Jan & 59.4 Aug & \\
\hline \multicolumn{13}{|l|}{ CHIPPEWA } \\
\hline $46 \mathrm{~N}$ 4W 24DADA1 & Raco & 54 & 6 & Qgd & 850 & 23 & $\mathrm{R}$ & 18.4 Jun 1971 & 28.4 Apr 1964 & 22.0 May & $25.7 \mathrm{Mar}$ & \\
\hline \multicolumn{13}{|l|}{ CLARE } \\
\hline $17 \mathrm{~N} 4 \mathrm{~W} 34 \mathrm{DCAD}$ & Clare & 91 & 4 & Qgd & 850 & 3 & $\mathrm{R}$ & 7.9 Mar 1976 & 22.2 Aug 1976 & $13.2 \mathrm{Dec}$ & 24.9 May & $\mathrm{P}$ \\
\hline
\end{tabular}


TABLE 1. RECORDS OF MICHIGAN OBSERVATION WELLS. (CONTINUED)

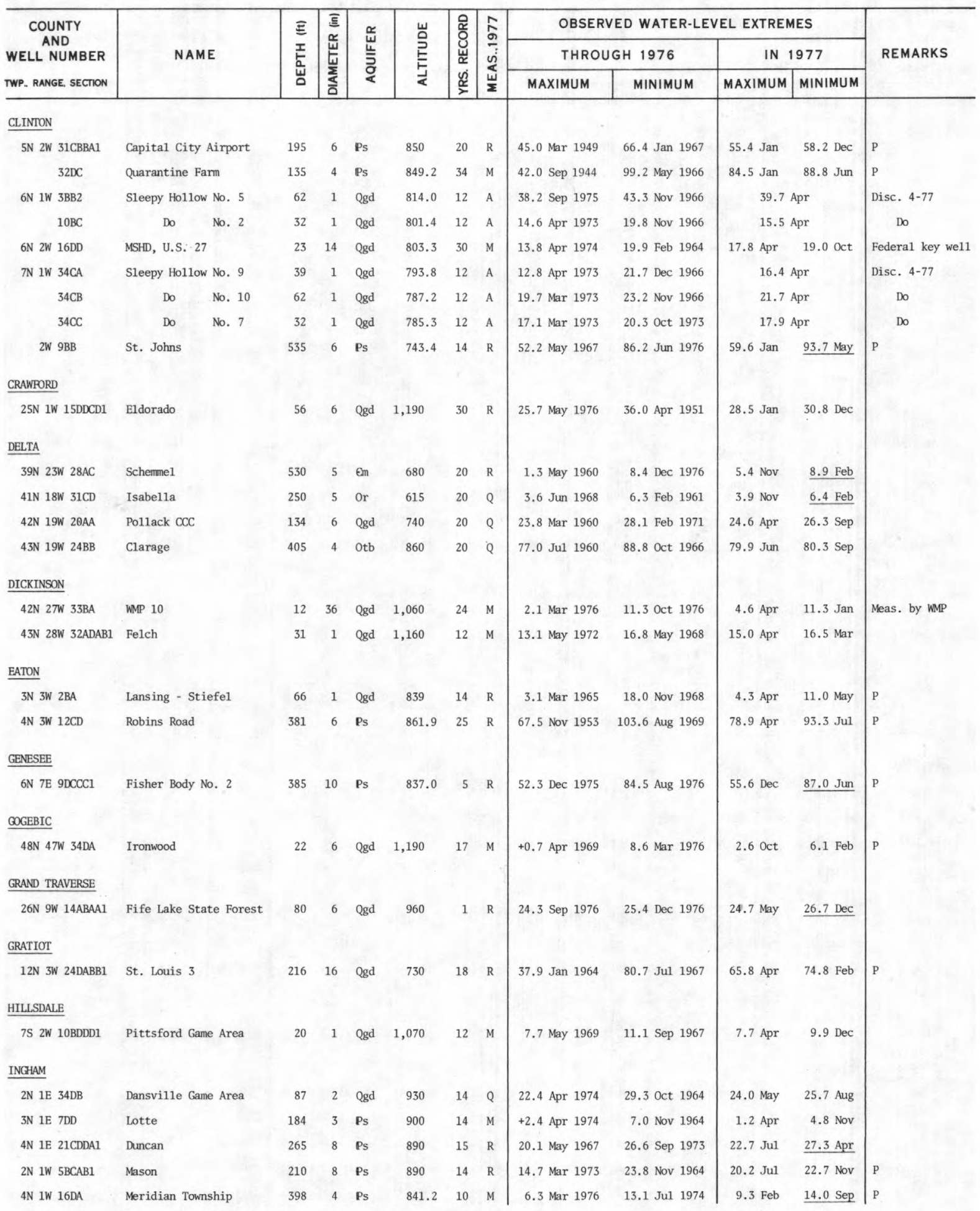


TABLE 1. RECORDS OF MICHIGAN OBSERVATION WELLS. (CONTINUED)

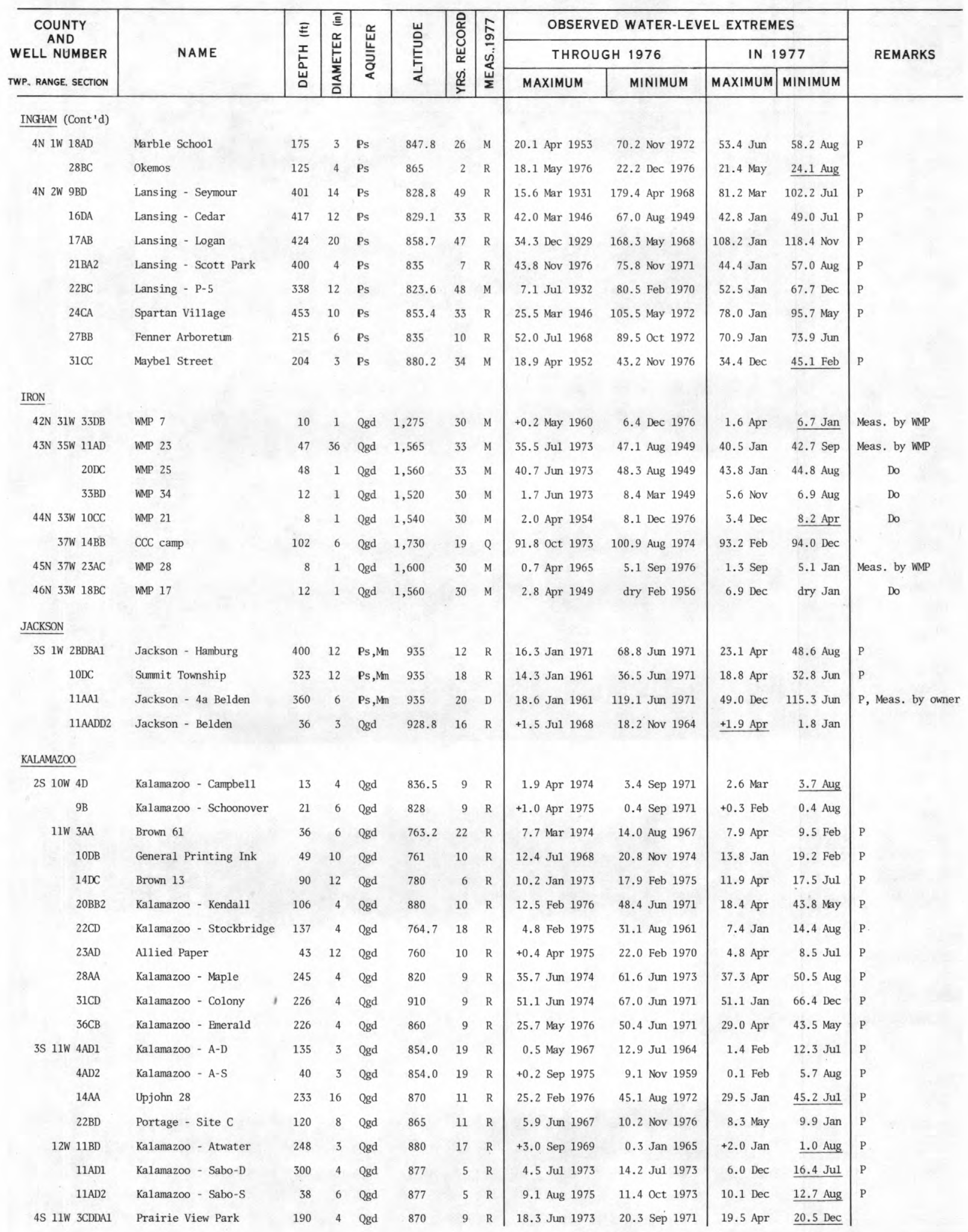


TABLE 1. RECORDS OF MICHIGAN OBSERVATION WELLS. (CONTINUED)

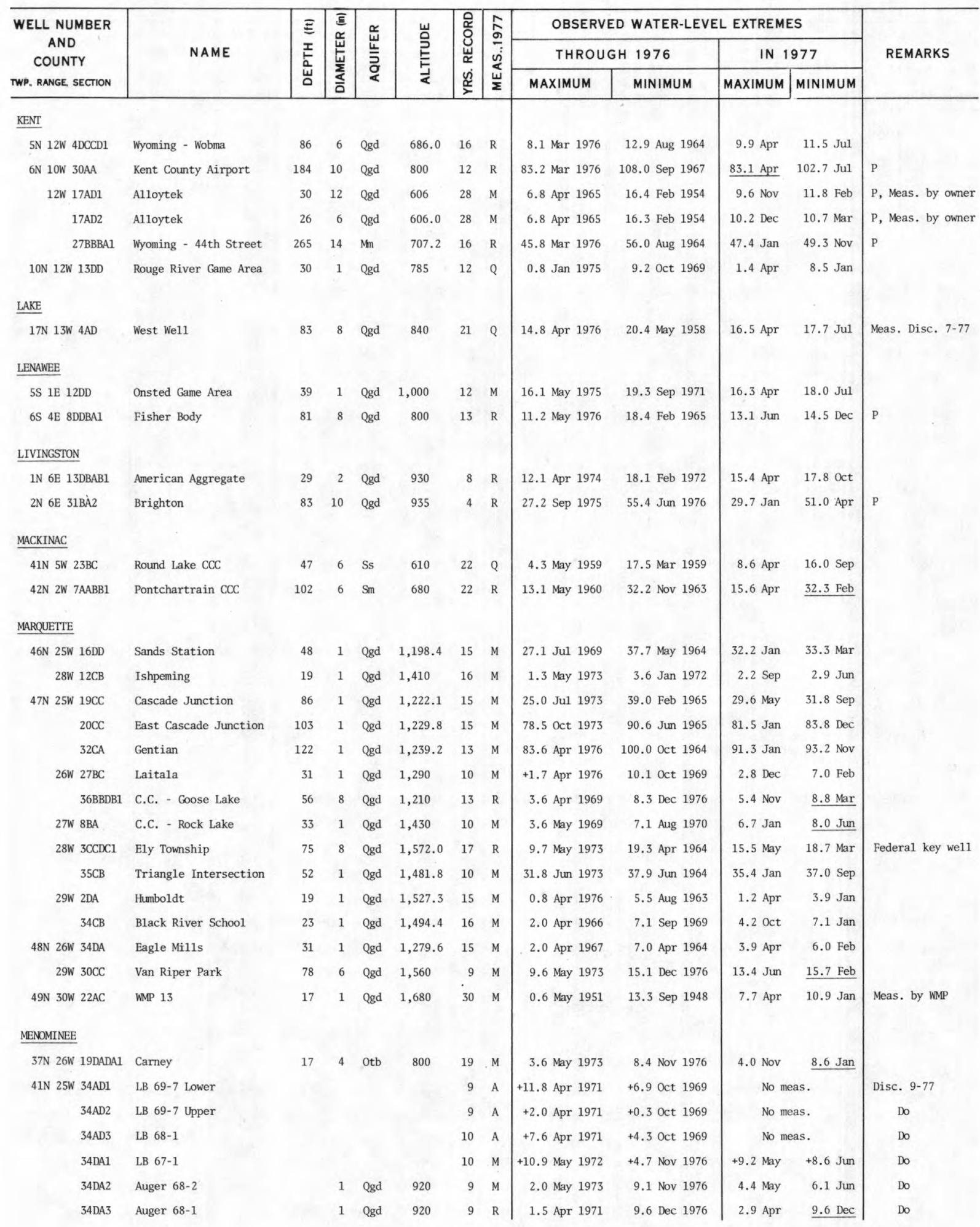


TABLE 1. RECORDS OF MICHIGAN OBSERVATION WELLS. (CONTINUED)

\begin{tabular}{|c|c|c|c|c|c|c|c|c|c|c|c|c|}
\hline \multirow{3}{*}{$\begin{array}{c}\text { COUNTY } \\
\text { AND } \\
\text { WELL NUMBER } \\
\text { TWP. RANGE, SECTION }\end{array}$} & \multirow{3}{*}{ NAME } & \multirow{3}{*}{$\begin{array}{l}\equiv \\
\underline{E} \\
\underline{\mathbf{5}} \\
\underline{u} \\
0\end{array}$} & \multirow{3}{*}{ 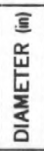 } & \multirow{3}{*}{ 皆 } & \multirow{3}{*}{ 岂 } & \multirow{3}{*}{ 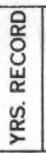 } & \multirow{3}{*}{ 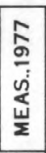 } & \multicolumn{4}{|c|}{ OBSERVED WATER-LEVEL EXTREMES } & \multirow{3}{*}{ REMARKS } \\
\hline & & & & & & & & \multicolumn{2}{|c|}{ THROUGH 1976} & \multicolumn{2}{|c|}{ IN 1977} & \\
\hline & & & & & & & & MAXIMUM & MINIMUM & MAXIMUM & MINIMUM & \\
\hline \multicolumn{13}{|l|}{ MENOMINEE (Cont'd) } \\
\hline 41N 25W 34DA4 & LB $68-4$ & & & & & 9 & A & 0.8 Apr 1971 & $3.1 \mathrm{Feb} 1970$ & \multicolumn{2}{|c|}{$\underline{5.2 \mathrm{Feb}}$} & Disc. $9-77$ \\
\hline $35 \mathrm{BA}$ & LB $69-4$ & & & & & 9 & A & +0.9 Jul 1969 & 0.6 Aug 1969 & \multicolumn{2}{|c|}{$\underline{3.3 \mathrm{Feb}}$} & Do \\
\hline $35 \mathrm{BB}$ & LB $69-5$ & & & & & 9 & A & 3.3 May 1969 & 4.7 Aug 1969 & \multicolumn{2}{|c|}{ 7.5 Feb } & Do \\
\hline $35 \mathrm{BC}$ & LB $69-1$ & & & & & 9 & A & 0.2 Apr 1971 & 2.0 Aug 1969 & \multicolumn{2}{|c|}{$\underline{5.0 \mathrm{Feb}}$} & Do \\
\hline \multicolumn{13}{|l|}{ MONROE } \\
\hline $7 \mathrm{~S} 6 \mathrm{E} 15 \mathrm{AD}$ & Petersburg Game Area & 17 & 1 & Qgd & 675 & 12 & M & 3.0 Feb 1966 & 6.7 Dec 1971 & 4.8 May & $6.6 \mathrm{Mar}$ & \\
\hline \multicolumn{13}{|l|}{ MUSKEGON } \\
\hline $11 N$ 15W 34ADDD1 & Muskegon Game Area & 31 & 1 & Qgd & 595 & 12 & $Q$ & 0.0 May 1974 & 4.7 Sep 1972 & $1.1 \mathrm{Apr}$ & $2.5 \mathrm{Jul}$ & \\
\hline \multicolumn{13}{|l|}{ OAKL.AND } \\
\hline $2 \mathrm{~N} 7 \mathrm{E} 5 \mathrm{BA}$ & Honeywe11 Lake Road & 44 & 2 & Qgd & 1,020 & 10 & $\mathrm{R}$ & 23.9 Apr 1976 & 28.9 Dec 1971 & $26.4 \mathrm{Apr}$ & 28.0 Sep & \\
\hline 8E 18DABAD1 & Proud Lake Park & 45 & 6 & Qgd & 910 & 9 & R & 2.8 May 1974 & 6.4 Sep 1971 & 4.9 Apr & 6.3 Aug & $\mathrm{P}$ \\
\hline 3N 7E 5DA & Fish Lake Road & 49 & 2 & Qgd & 1,055 & 9 & $\mathrm{R}$ & 29.5 Jun 1976 & 38.7 Dec 1972 & $32.3 \mathrm{Jan}$ & $34.7 \mathrm{Dec}$ & \\
\hline 8E 3DBAB1 & White Lake Road & 163 & 6 & Qgd & 1,000 & 6 & $\mathrm{R}$ & 7.2 May 1976 & 11.0 Sep 1972 & $8.8 \mathrm{Apr}$ & 11.0 Sep & \\
\hline $10 \mathrm{AB}$ & Teggerdine Road & 163 & 6 & Qgd & 1,000 & 6 & $\mathrm{R}$ & 27.8 Mar 1976 & 30.7 Sep 1972 & $28.8 \mathrm{Apr}$ & 30.5 Sep & \\
\hline 10E $13 \mathrm{AC}$ & Oakland University & 183 & 6 & Qgd & 940 & 17 & $\mathrm{R}$ & 57.1 May 1976 & 93.5 Jul 1963 & $57.1 \mathrm{Apr}$ & 58.2 oct & \\
\hline $5 \mathrm{~N} 8 \mathrm{E} 8 \mathrm{AC}$ & Holly Recreation Area & 42 & 1 & Qgd & 930 & 12 & M & 22.3 Apr 1974 & 26.5 Sep 1966 & $23.5 \mathrm{Apr}$ & 25.6 Aug & \\
\hline \multicolumn{13}{|l|}{ OGEMAW } \\
\hline 23N 1E $2 \mathrm{BAAA} 1$ & Rose City Road-D & 105 & 1 & Qgd & 1,265 & 10 & $Q$ & 73.6 oct 1976 & 78.2 Apr 1969 & 74.0 Jun & 74.7 Sep & \\
\hline 2BAAA2 & Rose City Road-S & 20 & 1 & Qgd & 1,265 & 10 & $Q$ & 7.6 Apr 1976 & 13.6 Dec 1972 & $11.0 \mathrm{Apr}$ & $12.4 \mathrm{Sep}$ & \\
\hline \multicolumn{13}{|l|}{ ONTONAGON } \\
\hline $46 \mathrm{~N} 38 \mathrm{~W}$ 30ADDD1 & USFS & 65 & 1 & Qgd & 1,530 & 11 & M & 16.0 Jun 1973 & 19.1 Apr 1970 & $18.2 \mathrm{Dec}$ & $19.6 \mathrm{Feb}$ & \\
\hline $\begin{array}{l}\text { 51N 41W 8BDBC1 } \\
\text { PRESQUE ISLE }\end{array}$ & Silver City & 100 & 6 & $\mathrm{p} \in f$ & 620 & 20 & M & 8.2 Apr 1959 & $21.8 \mathrm{Dec} 1976$ & 12.4 Oct & $21.3 \mathrm{Feb}$ & \\
\hline 33N 6E 8BBBB1 & Styma & 61 & 6 & $\mathrm{Dt}$ & 800 & 19 & Q & 5.4 Apr 1967 & 18.8 Mar 1963 & 7.6 Apr & $13.5 \mathrm{Jul}$ & 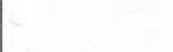 \\
\hline ROSCOMMON & & & & & & & & & & & & \\
\hline $24 \mathrm{~N} 2 \mathrm{~W} 20 \mathrm{BABA} \mathrm{I}$ & Exp. Station & 14 & 8 & Qgd & $1,145.3$ & 44 & $\mathrm{R}$ & 2.1 Apr 1976 & 6.2 Dec 1949 & $4.4 \mathrm{Apr}$ & $5.7 \mathrm{Feb}$ & Federal key well \\
\hline SANILAC & & & & & & & & & & & & \\
\hline 12N 13E 33DDDD1 & Elmer & 150 & 3 & $\mathrm{Mn}$ & 800 & 30 & w & 15.4 Apr 1951 & 25.6 Jan 1965 & $19.6 \mathrm{Dec}$ & $24.0 \mathrm{Sep}$ & \\
\hline 13N 13E 12ADAA & Minden Game Area & 130 & 6 & Mn & 805 & 1 & $\mathrm{R}$ & & & $18.1 \mathrm{Apr}$ & $21.9 \mathrm{Sep}$ & \\
\hline SCHOOLCRAFT & & & & & & & & & & & & \\
\hline $45 \mathrm{~N} 13 \mathrm{~W} 16 \mathrm{CCCB} 1$ & Seney & 154 & 4 & or & 710 & 26 & $\mathrm{R}$ & 4.6 Apr 1971 & 6.5 Oct 1963 & $5.2 \mathrm{Nov}$ & 6.0 Aug & \\
\hline $47 \mathrm{~N} 16 \mathrm{~W} 30 \mathrm{BBBB} 1$ & Cusino CCC & 57 & 6 & Op & 900 & 21 & $\mathrm{R}$ & 5.7 May 1960 & 16.3 Oct 1963 & $6.0 \mathrm{Apr}$ & $\underline{16.4 \mathrm{Feb}}$ & \\
\hline VAN BUREN & & & & & & & & & & & & \\
\hline $3 \mathrm{~S} 14 \mathrm{~W} 6 \mathrm{BA}$ & Martin & 59 & 1 & Qgd & 740 & 15 & M & 37.2 May 1974 & 43.3 Nov 1964 & $38.3 \mathrm{Apr}$ & 40.7 oct & \\
\hline 4S $16 \mathrm{~W} 14 \mathrm{CDBD} 1$ & Keeler & 170 & 14 & Qgd & 800 & 15 & M & 16.4 Ju1 1976 & 27.6 Aug 1964 & $18.8 \mathrm{Apr}$ & $20.8 \mathrm{Jul}$ & $\mathrm{P}$ \\
\hline $13 \mathrm{~W} 16 \mathrm{DD}$ & Porter Township Hal1 & 83 & 1 & Qgd & 930 & 15 & M & 40.4 Jun 1974 & 50.4 Oct 1964 & $41.7 \mathrm{Mar}$ & $43.2 \mathrm{Jan}$ & Disc. 8-77 \\
\hline
\end{tabular}


TABLE 1. RECORDS OF MICHIGAN OBSERVATION WELLS. (CONTINUED)

\begin{tabular}{|c|c|c|c|c|c|c|c|c|c|c|c|c|}
\hline \multirow{3}{*}{$\begin{array}{c}\text { COUNTY } \\
\text { AND } \\
\text { WELL NUMBER } \\
\text { TWP. RANGE, SECTION }\end{array}$} & \multirow{3}{*}{ NAME } & \multirow{3}{*}{ 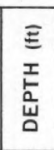 } & \multirow{3}{*}{ 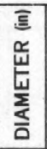 } & \multirow{3}{*}{ 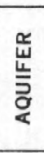 } & \multirow{3}{*}{ 㟧 } & \multirow{3}{*}{ 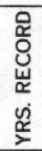 } & \multirow{3}{*}{ 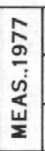 } & \multicolumn{4}{|c|}{ OBSERVED WATER-LEVEL EXTREMES } & \multirow{3}{*}{ REMARKS } \\
\hline & & & & & & & & \multicolumn{2}{|c|}{ THROUGH 1976} & \multicolumn{2}{|c|}{ IN 1977} & \\
\hline & & & & & & & & MAXIMUM & MINIMUM & MAXIMUM & MINIMUM & \\
\hline \multicolumn{13}{|l|}{ WASHTENAW } \\
\hline 2S 3E 9DAAB2 & Water1oo Park & 48 & 6 & Qgd & 970 & 9 & $\mathrm{R}$ & 4.1 May 1974 & 7.0 Aug 1971 & $5.0 \mathrm{Jun}$ & 6.6 Jan & P \\
\hline $3 \mathrm{~S} 6 \mathrm{E} 16 \mathrm{BCCD} 1$ & Ann Arbor & 55 & 10 & Qgd & 821.5 & 15 & $\mathrm{R}$ & 0.7 Mar 1974 & 15.9 Oct 1964 & $1.8 \mathrm{Apr}$ & 6.4 Sep & P \\
\hline $7 \mathrm{E} 5 \mathrm{BB}$ & Ypsilanti - Superior & 69 & 8 & Qgd & 720 & 16 & $\mathrm{R}$ & 1.8 Feb 1965 & 21.4 Dec 1965 & 5.5 Aug & $15.3 \mathrm{Feb}$ & P \\
\hline $9 \mathrm{AD}$ & Ypsilanti - Gilbert & 94 & 6 & Qgd & 710 & 27 & $\mathrm{R}$ & 29.1 Nov 1945 & 78.8 Oct 1974 & $71.3 \mathrm{Mar}$ & 77.5 Aug & p \\
\hline $24 \mathrm{CA} 1$ & Ypsilanti Township 104 & 87 & 4 & Qgd & 665.6 & 32 & $\mathrm{R}$ & 5.8 Jan 1950 & 22.7 Feb 1971 & 12.5 May & $15.7 \mathrm{Nov}$ & p \\
\hline $24 \mathrm{CD}$ & Ypsilanti Township 117 & 75 & 6 & Qgd & 657.8 & 31 & $\mathrm{R}$ & 5.7 Feb 1950 & 63.2 Feb 1970 & $5.7 \mathrm{Apr}$ & $16.7 \mathrm{Jul}$ & P \\
\hline \multicolumn{13}{|l|}{$\underline{\text { WEXFORD }}$} \\
\hline $21 \mathrm{~N} 9 \mathrm{~W}$ 4ABBC1 & Cadillac & 277 & 6 & Qgd & $1,291.1$ & 29 & Q & 19.4 Jun 1976 & 27.6 Jun 1964 & $20.5 \mathrm{Apr}$ & $21.8 \mathrm{Jul}$ & P \\
\hline $22 \mathrm{~N} 12 \mathrm{~W} 13 \mathrm{BA}$ & Harrietta Fish Hatchery & 141 & 4 & Qgd & 1,060 & 17 & $\mathrm{R}$ & +13.8 Mar 1970 & +1.5 Jan 1966 & +8.2 Dec & +4.2 Dec & P \\
\hline
\end{tabular}




\begin{tabular}{|c|c|c|c|c|c|c|c|c|c|c|c|c|c|c|c|}
\hline $\begin{array}{c}\text { COUNTY } \\
\text { AND } \\
\text { WATER USER }\end{array}$ & JAN & FEB & MAR & APR & MAY & JUN & JUL & AUG & SEP & OCT & NOV & $\mathrm{DEC}$ & $\begin{array}{r}1977 \\
\text { TOTAL }\end{array}$ & $\begin{array}{l}\text { MAX } \\
\text { DAY }\end{array}$ & $\begin{array}{l}\text { MIN } \\
\text { DAY }\end{array}$ \\
\hline \multicolumn{16}{|l|}{ ALCONA } \\
\hline Harrisville & 2.1 & 4.7 & 2.7 & 2.0 & 1.8 & 2.0 & 2.3 & 2.0 & 1.8 & 1.7 & 1.3 & 1.3 & 25.7 & - & .036 \\
\hline \multicolumn{16}{|l|}{ ALGER } \\
\hline $\begin{array}{l}\text { Burt Twp. } \\
\text { Chatham }\end{array}$ & $\begin{array}{r}1.9 \\
.8\end{array}$ & $\begin{array}{r}1.9 \\
.8\end{array}$ & $\begin{array}{r}2.4 \\
.7\end{array}$ & $\begin{array}{r}2.6 \\
.6\end{array}$ & $\begin{array}{r}5.9 \\
.9\end{array}$ & $\begin{array}{r}6.1 \\
.9\end{array}$ & $\begin{array}{r}5.6 \\
.9\end{array}$ & $\begin{array}{r}5.1 \\
.9\end{array}$ & $\begin{array}{r}3.2 \\
.8\end{array}$ & $\begin{array}{r}4.2 \\
.6\end{array}$ & $\begin{array}{r}2.0 \\
.6\end{array}$ & $\begin{array}{r}2.0 \\
.6\end{array}$ & $\begin{array}{r}42.9 \\
9.1\end{array}$ & $\begin{array}{l}-- \\
--\end{array}$ & 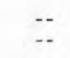 \\
\hline \multicolumn{16}{|l|}{ ALLEGAN } \\
\hline Allegan & 34.5 & 32.8 & 41.8 & 36.7 & 52.0 & 42.6 & 50.6 & 46.4 & 41.2 & 36.6 & 33.4 & 30.7 & 479.3 & 2.515 & .676 \\
\hline Douglas & 3.6 & 3.7 & 4.0 & 4.1 & 5.3 & 6.2 & 7.5 & 6.9 & 5.0 & 4.6 & 4.3 & 5.0 & 60.2 & .462 & .110 \\
\hline Fennville & 5.3 & 5.5 & 7.3 & 4.1 & 12.7 & 35.5 & 35.5 & 18.1 & 23.8 & 19.5 & 12.1 & 3.1 & 182.5 & -. & -- \\
\hline Otsego & 30.6 & 30.4 & 35.6 & 31.5 & 38.8 & 33.5 & 36.7 & 33.5 & 30.3 & 30.2 & 31.7 & 38.5 & 401.3 & 1.750 & .677 \\
\hline Plainwell & 10.2 & 10.7 & 11.3 & 11.5 & 12.0 & 14.6 & 20.0 & 15.1 & 14.8 & 12.9 & 10.1 & 12.3 & 155.5 & .979 & .212 \\
\hline Saugatuck & 2.9 & 6.0 & 9.7 & 7.2 & 10.4 & 9.8 & 11.9 & 10.3 & 8.3 & 6.2 & 7.5 & 5.3 & 95.5 & .762 & .109 \\
\hline \multicolumn{16}{|l|}{ ANTRIM } \\
\hline Bellaire & 3.8 & 3.7 & 4.0 & 4.0 & 6.5 & 5.9 & 5.5 & 4.6 & 4.1 & 3.8 & 3.7 & 3.6 & 53.2 & .396 & .102 \\
\hline Central Lake & 2.5 & 2.2 & $\begin{array}{l}4.0 \\
2.1\end{array}$ & $\begin{array}{l}4.0 \\
2.3\end{array}$ & $\begin{array}{l}0.3 \\
3.0\end{array}$ & $\begin{array}{l}5.9 \\
3.3\end{array}$ & $\begin{array}{l}3.5 \\
4.1\end{array}$ & $\begin{array}{l}4.0 \\
3.5\end{array}$ & $\begin{array}{l}4.1 \\
2.9\end{array}$ & $\begin{array}{l}3.8 \\
2.6\end{array}$ & $\begin{array}{l}3.1 \\
2.6\end{array}$ & $\begin{array}{l}3.0 \\
2.9\end{array}$ & 34.0 & $\begin{array}{l}.390 \\
.450\end{array}$ & .102 \\
\hline E11sworth & 1.0 & 1.0 & 1.0 & 1.0 & 1.4 & 1.6 & 1.6 & 1.4 & 1.3 & 1.2 & 1.3 & 1.1 & 14.9 & -2 & -- \\
\hline Mancelona & 12.4 & 13.4 & 15.0 & 10.8 & 19.9 & 18.6 & 18.3 & 13.9 & 13.6 & 16.2 & 14.0 & 13.3 & 179.4 & -- & -- \\
\hline \multicolumn{16}{|l|}{ BARRY } \\
\hline Hastings & 24.6 & 24.0 & 27.0 & 26.1 & 36.9 & 35.9 & 39.1 & 30.9 & 24.6 & 24.4 & 21.2 & 21.4 & 335.3 & 1.910 & .470 \\
\hline Middleville & 14.3 & 14.3 & 14.2 & 14.0 & 15.9 & 15.0 & 14.0 & 14.3 & 14.0 & 13.8 & 13.7 & 15.5 & 173.0 & -- & - \\
\hline Nashville & 3.9 & 5.6 & 6.2 & 3.9 & 4.5 & 4.2 & 4.5 & 3.6 & 3.6 & 3.3 & 2.8 & 3.8 & 49.9 & .251 & .068 \\
\hline \multicolumn{16}{|l|}{ BENZIE } \\
\hline Beulah' & 2.0 & 2.1 & 1.6 & 1.5 & 3.0 & 4.1 & 5.5 & 5.4 & 3.6 & 3.3 & 3.0 & 3.1 & 38.2 & -- & -. \\
\hline Frankfort & 11.6 & 11.0 & 12.5 & 9.5 & 10.5 & 11.6 & 9.1 & 9.6 & 9.0 & 8.0 & 7.1 & 6.8 & 116.3 & .592 & .197 \\
\hline \multicolumn{16}{|l|}{ BERRIEN } \\
\hline Berrien Springs & 12.0 & 13.0 & 14.0 & 11.9 & 20.1 & 18.0 & 19.0 & 15.6 & 12.6 & 12.8 & 11.3 & 11.6 & 171.9 & .978 & .248 \\
\hline Buchanan & 65.8 & 60.8 & 60.8 & 53.5 & 70.7 & 67.0 & 69.4 & 63.6 & 55.7 & 56.8 & 56.9 & 57.3 & 738.3 & 3.045 & 1.337 \\
\hline Coloma & 10.0 & 9.6 & 10.5 & 11.6 & 16.2 & 15.0 & 16.3 & 14.1 & 15.4 & 14.1 & 10.5 & 11.3 & 154.6 & .781 & .175 \\
\hline Niles & 88.4 & 83.6 & 88.4 & 90.7 & 115.5 & 99.7 & 100.3 & 97.2 & 86.6 & 91.8 & 81.3 & 85.5 & $1,109.0$ & 5.290 & 1.300 \\
\hline Niles Twp. & 3.9 & 3.4 & 3.4 & 3.7 & 9.9 & 7.3 & 8.3 & 5.4 & 4.0 & 3.4 & 3.6 & 4.5 & 60.8 & .610 & - \\
\hline Watervliet & 5.1 & 6.7 & 5.7 & 5.4 & 7.5 & 8.5 & 8.3 & 6.8 & 6.3 & 6.5 & 6.3 & 5.3 & 78.4 & .463 & .080 \\
\hline \multicolumn{16}{|l|}{ BRANCH } \\
\hline Bronson & 23.7 & 26.6 & 26.4 & 23.0 & 27.2 & 28.2 & 25.5 & 26.1 & 23.7 & 22.1 & 19.9 & 19.8 & 292.2 & 1.030 & .403 \\
\hline Coldwater & 82.6 & 77.4 & 87.0 & 87.9 & 125.3 & 116.0 & 123.1 & 108.2 & 89.7 & 88.2 & 86.7 & 82.2 & $1,154.3$ & 5.860 & 1.721 \\
\hline $\begin{array}{l}\text { Quincy } \\
\text { Reg. Center Dev. Disab. }\end{array}$ & 8.1 & 7.5 & 8.9 & 8.5 & 8.4 & 8.1 & 9.4 & 8.4 & 8.3 & 8.5 & 8.1 & 8.7 & $\begin{array}{l}\text { e) } 58.1 \\
100.9\end{array}$ & -- & $\begin{array}{l}-- \\
--\end{array}$ \\
\hline Union City & $\begin{array}{l}8.1 \\
4.9\end{array}$ & 8.3 & $\begin{array}{l}8.9 \\
9.5\end{array}$ & $\begin{array}{l}8.5 \\
6.9\end{array}$ & $\begin{array}{l}8.4 \\
6.7\end{array}$ & $\begin{array}{l}8.1 \\
7.4\end{array}$ & $\begin{array}{l}9.4 \\
8.1\end{array}$ & $\begin{array}{l}8.4 \\
5.8\end{array}$ & $\begin{array}{l}8.3 \\
4.7\end{array}$ & $\begin{array}{l}8.5 \\
3.8\end{array}$ & $\begin{array}{l}8.1 \\
3.7\end{array}$ & 3.5 & $\begin{array}{r}100.9 \\
73.3\end{array}$ & .521 & .103 \\
\hline \multicolumn{16}{|l|}{ CALHOUN } \\
\hline Albion & 101.6 & 99.6 & 104.4 & 74.8 & 114.2 & 109.9 & 111.8 & 107.8 & 108.9 & 119.4 & 100.1 & 99.1 & $1,251.6$ & 5.668 & 1.748 \\
\hline Athens & 1.0 & 3.4 & 5.2 & 2.9 & 2.3 & 5.0 & 7.3 & 4.9 & 4.0 & 3.3 & 3.0 & 2.5 & $\begin{array}{r}1,251.2 \\
45.2\end{array}$ & .171 & .028 \\
\hline Battle Creek & 170.3 & 186.0 & 203.5 & 199.1 & 240.3 & 256.6 & 255.1 & 214.2 & 191.2 & 181.5 & 175.5 & 169.6 & $2,442.9$ & 12.270 & 3.590 \\
\hline Battle Creek Twp. & 43.0 & 48.2 & 47.4 & 48.5 & 82.3 & 70.6 & 86.3 & 55.5 & 46.2 & 45.5 & 41.9 & 50.1 & 665.5 & 4.189 & 1.037 \\
\hline Homer & 6.1 & 7.0 & 6.3 & 5.8 & 6.9 & 5.7 & 5.7 & 6.0 & 5.7 & 5.9 & 4.7 & 5.0 & 70.8 & .438 & .098 \\
\hline Marshal1 & 33.7 & 31.9 & 34.4 & 33.6 & 45.3 & 40.9 & 45.1 & 43.7 & 36.0 & 36.9 & 33.4 & 34.3 & 449.2 & 2.029 & .701 \\
\hline \multicolumn{16}{|l|}{ CASS } \\
\hline Cassopolis & 3.1 & 2.9 & 3.1 & 3.2 & 3.5 & 3.9 & 3.7 & 4.1 & 3.7 & 3.5 & 3.1 & 3.1 & 40.9 & .287 & .091 \\
\hline Dowagiac & 30.7 & 29.6 & 33.2 & 25.4 & 39.6 & 31.7 & 38,0 & 31.6 & 32.3 & 22.1 & 23.0 & 26.4 & 363.6 & 2.096 & .354 \\
\hline Marcellus & 5.1 & 5.6 & 5.9 & 5.1 & 7.9 & 6.4 & 10.5 & 8.8 & 5.1 & 5.5 & 5.4 & 5.5 & 76.8 & -- & - \\
\hline \multicolumn{16}{|l|}{ CHARLEVOIX } \\
\hline Boyne City & 8.7 & 10.0 & 12.2 & 10.8 & 12.8 & 13.0 & 12.5 & 11.5 & 11.4 & 16.7 & 15.2 & 15.1 & 149.9 & .556 & .279 \\
\hline East Jordan & 17.4 & 18.7 & 21.5 & 18.8 & 25.9 & 20.9 & 22.8 & 21.9 & 22.3 & 20.6 & 18.8 & 18.5 & 248.1 & 1.080 & .400 \\
\hline \multicolumn{16}{|l|}{ CHEBOYGAN } \\
\hline Cheboygan & 48.5 & 64.4 & 72.9 & 70.0 & 79.6 & 86.4 & 94.9 & 69.4 & 7.7 & 5.1 & 22.0 & 13.8 & 634.7 & 2.873 & .197 \\
\hline Mackinaw City & 2.1 & 5.0 & 5.2 & 5.6 & 7.9 & $\begin{array}{r}0.4 \\
7.7\end{array}$ & 11.4 & 11.3 & 9.3 & 4.3 & 3.0 & $\begin{array}{r}10.8 \\
2.8\end{array}$ & 75.6 & .882 & .058 \\
\hline \multicolumn{16}{|l|}{ CHIPPEWA } \\
\hline Kincheloe AFB & 31.5 & 27.4 & 30.5 & 29.9 & 37.0 & 34.2 & 27.1 & 16.9 & 12.5 & 10.1 & 5.6 & 6.8 & 269.5 & 1.861 & .007 \\
\hline
\end{tabular}


TABLE 2. REPORTED GROUND-WATER PUMPAGE, IN 1977. (IN MILLIONS OF GALLONS)-continued

\begin{tabular}{|c|c|c|c|c|c|c|c|c|c|c|c|c|c|c|c|}
\hline $\begin{array}{c}\text { COUNTY } \\
\text { AND } \\
\text { WATER USER }\end{array}$ & JAN & FEB & MAR & APR & MAY & JUN & JUL & AUG & SEP & OCT & NOV & DEC & $\begin{array}{r}1977 \\
\text { TOTAL }\end{array}$ & $\begin{array}{l}\text { MAX } \\
\text { DAY }\end{array}$ & $\begin{array}{l}\text { MIN } \\
\text { DAY }\end{array}$ \\
\hline \multicolumn{16}{|l|}{ CLARE } \\
\hline Clare & 20.9 & 21.2 & 26.9 & 24.6 & 59.3 & 42.2 & 37.2 & 27.2 & $\begin{array}{r}23.0 \\
3.8\end{array}$ & 22.5 & 20.7 & 21.1 & $\begin{array}{r}346.8 \\
59.7\end{array}$ & 2.710 & .534 \\
\hline Harrison & $\begin{array}{l}4.2 \\
7.3\end{array}$ & $\begin{array}{l}4.4 \\
9.4\end{array}$ & $\begin{array}{l}4.4 \\
8.6\end{array}$ & $\begin{array}{l}4.1 \\
6.7\end{array}$ & $\begin{array}{l}9.4 \\
7.1\end{array}$ & $\begin{array}{r}7.8 \\
16.4\end{array}$ & $\begin{array}{r}0.5 \\
16.5\end{array}$ & $\begin{array}{l}4.6 \\
9.4\end{array}$ & $\begin{array}{l}3.8 \\
7.7\end{array}$ & $\begin{array}{l}3.4 \\
7.5\end{array}$ & $\begin{array}{l}3.3 \\
7.6\end{array}$ & $\begin{array}{l}3.8 \\
7.6\end{array}$ & $\begin{array}{r}59.7 \\
111.8\end{array}$ & $\begin{array}{l}.450 \\
.914\end{array}$ & $\begin{array}{l}.079 \\
.192\end{array}$ \\
\hline \multicolumn{16}{|l|}{ CLINTON } \\
\hline Fowler & 2.2 & 2.4 & 2.7 & 2.5 & 2.2 & 2.4 & 2.8 & 2.2 & 2.2 & 2.8 & 2.9 & 3.2 & 30.5 & -- & -- \\
\hline Maple Rapids & 1.4 & 1.5 & 1.9 & 1.7 & 1.5 & 1.4 & 2.1 & 3.0 & 3.4 & 1.6 & 1.6 & 1.5 & 22.6 & $=$ & $\because-$ \\
\hline Ovid & 8.7 & 6.7 & 5.9 & 5.0 & 5.7 & 5.0 & 5.2 & 4.3 & 5.7 & 3.4 & 5.6 & 8.2 & 69.4 & .393 & 103 \\
\hline St. Johns & 49.4 & 50.7 & 49.9 & 46.0 & 59.0 & 53.9 & 55.3 & 57.4 & 50.8 & 50.7 & 41.6 & 42.7 & 607.4 & 2.860 & .860 \\
\hline Westphalia & 2.2 & 1.9 & 1.7 & 2.1 & 2.6 & 2.2 & 1.2 & 1.6 & 1.8 & 1.8 & 1.7 & 1.6 & 22.4 & .160 & .026 \\
\hline \multicolumn{16}{|l|}{ CRAWFORD } \\
\hline Grayling & 13.9 & 15.0 & 16.3 & 17.1 & 21.7 & 22.8 & 25.6 & 21.6 & 19.0 & 19.9 & 20.1 & 20.9 & 233.9 & 1.077 & .392 \\
\hline \multicolumn{16}{|l|}{ DICKINSON } \\
\hline Breitung Twp. & 2.1 & 2.3 & 2.6 & 2.4 & 3.5 & 2.9 & 3.1 & 2.7 & 2.5 & 2.4 & 2.4 & 2.4 & 31.3 & .187 & .030 \\
\hline \multicolumn{16}{|l|}{ EATON } \\
\hline Bellevue & 4.0 & 5.0 & 5.4 & 4.0 & 5.0 & 4.7 & 5.8 & 5.4 & 3.8 & 4.5 & 3.7 & 3.2 & 54.5 & .250 & .015 \\
\hline Charlotte & 38.9 & 37.2 & 39.1 & 37.8 & 46.4 & 42.8 & 43.7 & 38.6 & 37.2 & 38.2 & 35.4 & 36.9 & 472.2 & 1.855 & .006 \\
\hline Delta Twp. & 48.3 & 46.5 & 44.8 & 43.5 & 76.6 & 65.0 & 67.1 & 63.4 & 50.6 & 48.0 & 45.5 & 45.7 & 645.0 & 3.664 & .848 \\
\hline Eaton Rapids & 21.4 & 22.1 & 29.1 & 23.6 & 28.9 & 29.6 & 28.5 & 29.6 & 30.7 & 23.8 & 24.7 & 23.3 & 315.3 & 2.199 & .699 \\
\hline Grand Ledge & 19.6 & 21.2 & 18.8 & 16.6 & 23.0 & 20.1 & 23.3 & 16.9 & 15.3 & 16.7 & 16.0 & 15.9 & 223.4 & -- & -- \\
\hline Olds Warehouse & .8 & .8 & .7 & .7 & .9 & .8 & 1.2 & 1.2 & .8 & 1.1 & .7 & .6 & 10.3 & -- & -- \\
\hline Olivet & 4.3 & 4.2 & 4.2 & 3.8 & 3.5 & 2.9 & 4.1 & 4.5 & 4.6 & 5.3 & 4.7 & 4.5 & 50.6 & .531 & -- \\
\hline Sunfield & 1.8 & 1.4 & 2.2 & 2.6 & 4.2 & 3.2 & 3.1 & 2.2 & 2.0 & 1.9 & 2.0 & 2.0 & 28.6 & $-\cdot$ & $-\cdot$ \\
\hline \multicolumn{16}{|l|}{ EMET } \\
\hline Harbor Springs & 11.6 & 10.5 & 12.8 & 10.0 & 22.9 & 26.9 & 28.9 & 19.4 & 13.5 & 11.5 & 10.3 & 10.4 & 188.7 & 1.521 & .298 \\
\hline Petosky & 41.1 & 37.5 & 41.5 & 37.9 & 43.4 & 43.2 & 49.0 & 45.9 & 37.9 & 41.2 & 36.0 & 40.6 & 495.2 & 1.865 & -- \\
\hline \multirow{2}{*}{\multicolumn{16}{|c|}{ GENESEE }} \\
\hline & & 40.8 & 36.9 & 34.8 & 46.9 & 41.0 & 45.9 & 38.5 & 36.0 & 37.3 & 33.6 & 37.1 & 471.0 & 2.185 & 939 \\
\hline Burton & 13.8 & 15.7 & 21.5 & 22.7 & 35.4 & 29.5 & 33.1 & 26.4 & 25.0 & 23.2 & 22.3 & 20.9 & 289.5 & 1.898 & - \\
\hline Davison & .22 .8 & 21.1 & 21.1 & 19.3 & 20.8 & 31.1 & 26.8 & 32.1 & 22.2 & 21.9 & 23.8 & 21.5 & 284.5 & 2.583 & .068 \\
\hline Fenton & 25.0 & 26.5 & 33.5 & 27.7 & 38.2 & 33.0 & 35.4 & 30.0 & 28.1 & 26.4 & 25.1 & 27.2 & 356.1 & 1.785 & .447 \\
\hline Grand Blanc & 26.3 & 35.4 & 32.5 & 29.5 & 45.0 & 47.8 & 50.7 & 38.4 & 32.3 & 32.7 & 31.5 & 27.5 & 429.6 & 2.713 & .850 \\
\hline $\begin{array}{l}\text { Grand Blanc Twp. } \\
\text { Linden }\end{array}$ & & & & & & & & & & & & & $\begin{array}{r}\text { e) } 18.2 \\
51.9\end{array}$ & -- & $\because-$ \\
\hline Montrose & 5.7 & 5.1 & 5.0 & 4.8 & 6.6 & 6.0 & 7.2 & 5.5 & 5.2 & 5.4 & 4.8 & 4.8 & 66.1 & .520 & .096 \\
\hline Otisville & 1.4 & 1.7 & 1.2 & 1.2 & 1.9 & 1.4 & 1.5 & .8 & 1.2 & 1.3 & 1.1 & 1.1 & 15.8 & .080 & .026 \\
\hline \multicolumn{16}{|l|}{ GLADWIN } \\
\hline Beaverton & 3.8 & 4.9 & 4.6 & 3.6 & 4.5 & 4.5 & 3.8 & 3.9 & 3.1 & 3.4 & 3.3 & 3.2 & 46.6 & -. & -- \\
\hline \multicolumn{16}{|l|}{ GOGEBIC } \\
\hline Ironwood & 48.0 & 52.8 & 53.3 & 46.9 & 46.4 & 41.4 & 45.4 & 43.7 & 40.4 & 41.2 & 39.9 & 40.5 & 539.9 & -- & -- \\
\hline Marenisco Twp. & 6.7 & 6.6 & 6.9 & 5.3 & 6.3 & 5.5 & 5.6 & 5.2 & 4.3 & 4.2 & 5.2 & 6.6 & 68.4 & .332 & .112 \\
\hline Wakefield & 9.6 & 8.9 & 9.0 & 7.6 & 9.7 & 8.5 & 9.0 & 8.7 & 8.4 & 8.8 & 9.9 & 9.8 & 106.9 & 1.117 & .212 \\
\hline Watersmeet & 1.6 & 1.4 & 1.6 & 1.5 & 1.6 & 1.5 & 1.6 & 1.6 & 1.5 & 1.6 & 1.5 & 1.6 & e) 18.6 & 列 & -- \\
\hline \multicolumn{16}{|l|}{ GRAND TRAVERSE } \\
\hline Kingsley & 1.5 & 1.5 & 1.5 & 1.6 & 2.1 & 3.3 & 3.3 & 2.2 & 1.8 & 1.6 & 1.5 & 1.5 & 23.4 & .109 & .041 \\
\hline \multicolumn{16}{|l|}{ GRATIOT } \\
\hline a)Alma & $=$ & $\because-$ & 26.8 & 5.0 & -- & -- & -- & -- & 29.7 & 9.3 & -- & -- & 70.8 & -- & -- \\
\hline Breckenridge & 3.3 & 3.3 & 3.3 & 3.2 & 4.0 & 3.4 & 3.5 & 3.1 & 3.1 & 3.2 & 2.9 & 3.1 & 39.4 & .180 & .070 \\
\hline Ithaca & 7.8 & 8.0 & 5.6 & 7.4 & 9.9 & 8.2 & 6.2 & 10.2 & 7.7 & 7.3 & 5.1 & 8.5 & 91.9 & .432 & .146 \\
\hline St. Louis & 50.3 & 43.5 & 44.1 & 38.1 & 47.6 & 48.6 & 44.2 & 48.8 & 46.6 & 43.3 & 37.9 & 39.9 & 532.9 & 2.102 & 1.160 \\
\hline \multicolumn{16}{|l|}{ HILLSDALE } \\
\hline Camden & & 1.4 & 1.3 & & & 1.5 & 1.6 & 1.5 & 1.2 & 1.3 & 1.1 & 1.1 & 16.4 & -- & -- \\
\hline Hillsdale & 38.8 & 40.1 & 44.0 & 41.2 & 47.7 & 46.0 & 45.4 & 40.4 & 43.0 & 41.9 & 39.8 & 45.2 & 513.5 & 1.881 & .952 \\
\hline Jonesville & 15.6 & 17.0 & 16.8 & 14.2 & 16.9 & 16.4 & 16.7 & 14.7 & 14.4 & 15.1 & 13.9 & 18.9 & 190.6 & .870 & .228 \\
\hline Litchfield & 3.3 & 4.8 & 4.5 & 3.8 & 3.9 & 5.4 & 4.3 & 4.8 & 3.8 & 3.4 & 3.7 & 3.8 & 49.5 & .251 & .084 \\
\hline Waldron & 1.8 & 2.0 & 1.9 & 1.7 & 2.3 & 2.1 & 2.1 & 2.0 & 1.8 & 1.7 & 1.7 & 1.9 & 23.0 & .142 & .015 \\
\hline
\end{tabular}


TABLE 2. REPORTED GROUND-WATER PUMPAGE, IN 1977. (IN MILLIONS OF GALLONS)-CONTINUED

\begin{tabular}{|c|c|c|c|c|c|c|c|c|c|c|c|c|c|c|c|}
\hline $\begin{array}{c}\text { COUNTY } \\
\text { AND } \\
\text { WATER USER }\end{array}$ & JAN & FEB & MAR & APR & MAY & JUN & JUL & AUG & SEP & OCT & NOV & DEC & $\begin{array}{r}1977 \\
\text { TOTAL }\end{array}$ & $\begin{array}{l}\text { MAX } \\
\text { DAY }\end{array}$ & $\begin{array}{l}\text { MIN } \\
\text { DAY }\end{array}$ \\
\hline \multicolumn{16}{|l|}{ HOUGHTON } \\
\hline $\begin{array}{l}\text { W) Adams } \\
\text { Water Auth. } \\
\text { c) Adams Twp. - S. Range }\end{array}$ & 63.8 & 60.0 & 64.7 & 63.2 & 63.4 & 62.4 & 62.7 & 62.5 & 61.7 & 64.8 & 60.0 & 60.8 & e) 750.0 & -- & -- \\
\hline Water Auth. & 9.7 & 10.7 & 11.5 & 11.8 & 10.9 & 9.0 & 9.0 & 8.0 & 8.1 & 8.5 & 8.3 & 8.1 & e) 113.6 & & \\
\hline Chasse11 Twp. & 4.8 & $\begin{array}{r}4.5 \\
32\end{array}$ & 4.5 & 3.2 & 3.0 & 2.9 & $\begin{array}{r}3.3 \\
36\end{array}$ & 3.2 & 2.8 & 2.6 & $\begin{array}{r}2.4 \\
33.8\end{array}$ & $\begin{array}{r}2.7 \\
34.9\end{array}$ & 39.9 & .167 & .073 \\
\hline $\begin{array}{l}\text { Hougnton } \\
\text { d) N. Michigan Water Co. }\end{array}$ & $\begin{array}{l}35.8 \\
34.5\end{array}$ & $\begin{array}{l}32.7 \\
39.1\end{array}$ & $\begin{array}{l}34.2 \\
44.6\end{array}$ & $\begin{array}{l}41.4 \\
38.2\end{array}$ & $\begin{array}{l}42.0 \\
38.0\end{array}$ & $\begin{array}{l}37.1 \\
36.8\end{array}$ & $\begin{array}{l}36.7 \\
38.6\end{array}$ & $\begin{array}{l}33.9 \\
38.7\end{array}$ & $\begin{array}{l}37.2 \\
34.3\end{array}$ & $\begin{array}{l}38.5 \\
33.9\end{array}$ & $\begin{array}{l}33.8 \\
32.3\end{array}$ & $\begin{array}{l}34.9 \\
32.5\end{array}$ & 441.5 & $\begin{array}{l}1.511 \\
1.783\end{array}$ & .786 \\
\hline \multicolumn{16}{|l|}{ HURON } \\
\hline E1kton & 2.4 & 3.0 & 2.8 & 2.4 & 2.9 & 2.7 & 3.0 & 2.5 & 1.9 & 2.3 & 2.0 & 2.0 & 29.9 & .118 & .020 \\
\hline $\begin{array}{l}\text { Q) Pigeon } \\
\text { Sebewaing }\end{array}$ & 9.2 & & $\begin{array}{r}6.4 \\
13.1\end{array}$ & & 9.5 & $\begin{array}{r}6.9 \\
13.0\end{array}$ & & & $\begin{array}{r}8.7 \\
10.4\end{array}$ & 11.0 & 10.8 & $\begin{array}{r}7.6 \\
12.4\end{array}$ & $\begin{array}{r}29.6 \\
140.9\end{array}$ & $\begin{array}{l}-- \\
-\end{array}$ & $\begin{array}{l}-- \\
-.\end{array}$ \\
\hline & & & & & & & & & & & & & & & \\
\hline \multicolumn{16}{|l|}{ INGHAM } \\
\hline E. Lansing-Meridian Twp. & 133.1 & 121.1 & 126.4 & 128.3 & 178.3 & 154.2 & 164.9 & 143.3 & 125.1 & 134.1 & 124.2 & 120.6 & $1,653.6$ & 8.781 & 2.599 \\
\hline Lansing & 740.3 & 677.3 & 750.9 & 737.3 & 923.6 & 878.8 & 824.9 & 00.1 & 16.3 & 752.7 & 714.4 & 696.3 & $9,202.9$ & 37.660 & 16.590 \\
\hline Lansing Twp. & 56.7 & 47.8 & 50.8 & 44.2 & 42.6 & 57.3 & 21.0 & 35.4 & 43.9 & 38.0 & 36.6 & 33.3 & 507.6 & -. & -- \\
\hline Les1 & 7.6 & 6.1 & 6.8 & 6.7 & 11.3 & 9.6 & 9.9 & 8.4 & 7.0 & 6.7 & 6.3 & 6.8 & 93.2 & -. & -. \\
\hline Mason & 28.0 & 14.0 & 15.5 & 4.3 & 23.0 & 19.9 & 19.9 & 18.6 & 14.5 & 18.2 & 14.4 & 16.1 & 20 & -- & $\ddot{-}$ \\
\hline Michigan State Univ. & 140.5 & 131.1 & 135.2 & 145.0 & 174.9 & 144.9 & 149.9 & 129.7 & 117.2 & 156.4 & 136.8 & 113.4 & $1,675.0$ & 6.231 & 1.967 \\
\hline Oldsmobile Forge No. 2 & 7.4 & 7.7 & 7.0 & 15.9 & 17.6 & 15.0 & 8.5 & 14.0 & 12.5 & 11.2 & 10.6 & 10.2 & 137.6 & $\bar{z}$ & -- \\
\hline Stockbridge & 3.7 & 3.1 & 4.0 & 4.0 & 4.7 & 4.2 & 3.9 & 4.0 & 3.5 & 3.5 & 3.1 & 3.2 & 44.9 & .266 & .081 \\
\hline Webberville & 3.0 & 3.4 & 3.8 & 4.6 & 7.1 & 5.6 & 5.7 & 4. & 3.3 & 3.3 & 3.2 & 3.1 & 50.1 & .330 & .093 \\
\hline Williamston & 10.3 & 9.2 & 8.3 & 7.3 & 8.8 & 9.0 & 9.1 & 8.7 & 7.2 & 7.3 & 6.6 & 6.8 & 98.6 & .439 & .188 \\
\hline \multicolumn{16}{|l|}{ IONIA } \\
\hline Belding & 38.3 & 33.2 & 45.4 & 46.5 & 52.5 & 53.6 & 55.9 & 48.0 & 36.8 & 46.8 & 44.8 & 44.4 & 546.2 & $\cdots$ & -. \\
\hline $\begin{array}{l}\text { Ionia } \\
\text { Inia }\end{array}$ & 30.1 & 29.8 & 29.2 & 28.1 & 36.9 & 32.3 & 35.7 & 32.4 & 27.7 & 26.8 & 24.9 & 23.6 & 357.5 & 1.623 & .550 \\
\hline Mich. Reformatory, Ionia & 20.4 & 16.6 & 17.2 & 19.4 & 18.5 & 18.8 & 15.7 & 14.4 & 17.0 & 16.3 & 17.2 & 17.7 & 209.2 & -. & -- \\
\hline Mich. Training Unit, Ionia & 5.6 & 4.4 & 5.6 & 7.2 & 9.7 & 6.3 & 9.4 & 6.2 & 6.2 & 5.5 & 5.3 & 5.3 & 76.7 & .501 & .128 \\
\hline Muir & 1.2 & 1.2 & 1.2 & 1.2 & 1.2 & 3.3 & 7.2 & 3 . & 2.7 & 3.6 & 2. & 3.2 & 32.2 & .867 &.- \\
\hline Pewamo & 1.2 & 1.1 & 1.1 & & 3.3 & 2.4 & 3.9 & 1.5 & 1 & 1 & & 1.1 & 20.1 & - & -. \\
\hline Portlan & 13.2 & 12.2 & 12.7 & 12.9 & 15.6 & 16.0 & 13.5 & 14.5 & 13. & 13.3 & 13 & 13.1 & 163.5 & -- & -- \\
\hline Riverside Center & 2.4 & 2.9 & 2. & 1.9 & 3.6 & 2.8 & 3. & 2. & 2. & & 2 & 2. & 31.1 & .192 & .052 \\
\hline Saranac & 10.8 & 10.0 & 11.3 & 10.1 & 13.2 & 13.0 & 13.6 & 13.4 & 13.1 & 12.9 & 12.4 & 12.8 & 146.6 & .671 & .122 \\
\hline \multicolumn{16}{|l|}{ IOSCO } \\
\hline Oscoda Twp. & 16.6 & 20.2 & 21.3 & 16.0 & 23.0 & 22.6 & 26.6 & 19.2 & 13.4 & 8.8 & 11.2 & 9.0 & 207.9 & $=-$ & 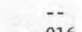 \\
\hline Wurtsmith AFB & 18.1 & 17.0 & 19.4 & 17.5 & 30.0 & 33.1 & 35.8 & 21.4 & 16.7 & 19.4 & 17.2 & 17.2 & 262.8 & 1.703 & .016 \\
\hline \multicolumn{16}{|l|}{ IRON } \\
\hline Alph & & .8 & 9 & & & 8 & .9 & 5 & .8 & & .8 & & 0.3 & -- & 153 \\
\hline Casp & & 9.7 & 10.3 & & 10.3 & 10.3 & 12.1 & & 12.1 & 11.7 & $\begin{array}{l}10.1 \\
12.3\end{array}$ & 10.7 & $\begin{array}{l}130.4 \\
165.1\end{array}$ & $\begin{array}{l}.659 \\
.832\end{array}$ & .153 \\
\hline Fal1s & & 13.5 & 14.3 & 13.0 & 16.4 & 14.6 & 16.5 & 13.8 & $\begin{array}{r}12.1 \\
6.0\end{array}$ & $\begin{array}{r}12.7 \\
6.9\end{array}$ & 6. & $\begin{array}{r}13 \\
6\end{array}$ & $\begin{array}{r}165.1 \\
81.8\end{array}$ & .832 & .00 \\
\hline $\begin{array}{l}\text { Falls Twp. } \\
\text { ver }\end{array}$ & $\begin{array}{r}5 \\
11\end{array}$ & $\begin{array}{r}6.6 \\
14.7\end{array}$ & $\begin{array}{r}6.9 \\
13.6\end{array}$ & $\begin{array}{r}7.5 \\
11.8\end{array}$ & $\begin{array}{r}7.6 \\
10.9\end{array}$ & $\begin{array}{r}7.1 \\
10.0\end{array}$ & $\begin{array}{r}7.8 \\
11.4\end{array}$ & $\begin{array}{r}7 \\
10\end{array}$ & $\begin{array}{l}6 . \\
9\end{array}$ & $\begin{array}{l}6.9 \\
9.7\end{array}$ & 6. & $\begin{array}{r}6 \\
10\end{array}$ & $\begin{array}{r}81.8 \\
133.0\end{array}$ & .689 & .209 \\
\hline $\begin{array}{l}\text { Iron River } \\
\text { Iron River Twp. }\end{array}$ & $\begin{array}{l}11.3 \\
11.7\end{array}$ & $\begin{array}{l}14.7 \\
12.6\end{array}$ & $\begin{array}{l}12.0 \\
12.6\end{array}$ & 11. & 14. & 12. & 13. & 11. & 9 & 8.9 & & 10 & 137.6 & - & 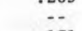 \\
\hline Stamb & 4. & 5. & 5.9 & 4. & 5 & 4. & 6 & 5 & 4. & 5. & & 6 & 65.0 & .367 & .131 \\
\hline Stambaugh Twp. & 4.2 & 5.8 & 5.8 & 4.0 & 4.5 & 4.4 & 5.2 & 4. & 3.2 & 3.3 & 3. & 3. & 52.0 & -- & 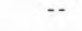 \\
\hline \multicolumn{16}{|l|}{ ISABELLA } \\
\hline f) Mt. P1easant & 69.8 & 77.8 & 78.3 & 76.2 & 93.6 & 80.0 & 78.8 & 79.9 & 77.2 & 77.2 & 72.6 & 65.9 & 927.3 & -- & 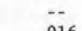 \\
\hline Shepherd & 3.1 & 3.5 & 4.4 & 4.6 & 3.9 & 4.3 & 6.4 & 4.2 & 4.3 & 4.5 & 4.8 & 4.2 & 52.2 & .287 & .016 \\
\hline \multicolumn{16}{|l|}{ JACKSON } \\
\hline Concord & & & & & & & & & & & & & & .502 & \\
\hline Cake & & 5.3 & & & & & & & 1.6 & & & & & 1.471 & .030 \\
\hline Jac & 370.5 & 342.5 & 360.2 & 343.8 & 404.1 & 399.9 & 377.6 & 406.1 & 348.8 & 341.2 & 323.2 & 312 & $4,330.2$ & 16.780 & 6.370 \\
\hline & 3 & 3. & 3.5 & 3 & 3.8 & 4.9 & 4.7 & 3.8 & 3.8 & 3.7 & 3. & & 44.9 & & \\
\hline State Prison, Jackson & 44.4 & 35.8 & 42.6 & 42.5 & 47.5 & 45.3 & 48.5 & 48.1 & 46.4 & 48.5 & 45.5 & 43.2 & 538.3 & 1.810 & 1.300 \\
\hline \multicolumn{16}{|l|}{ KALAL } \\
\hline & 2.1 & 3.6 & 3.8 & 2.1 & 2.9 & 3.0 & 2.5 & 2.3 & 2.2 & 2.6 & 3.0 & 3.2 & 3 & .182 & .038 \\
\hline & 301.9 & 281 & 312.1 & 2 & 279.8 & 275.7 & 261.4 & 278 & 265 & 290 & 279 & & & & \\
\hline $\mathrm{G}$ & 5.5 & 4.9 & 5.3 & 0.0 & 8.4 & 8.3 & & ' & 5 & & 3 & 3 & 75.9 & .598 & .101 \\
\hline & 449.2 & 439.0 & 470.4 & 504.7 & 706.9 & 637.2 & 790.9 & 599.9 & 512.8 & 485.9 & 431.1 & 447.1 & $6,475.7$ & 34.606 & 8.653 \\
\hline $\mathrm{P}_{\mathrm{i}}$ & 7.7 & 6.9 & 7.7 & 8.1 & 15.4 & 12.6 & 15.7 & 9.9 & 8.0 & 7.4 & 6.7 & 6.7 & 112.8 & 1.178 & .288 \\
\hline & 53.7 & 52.4 & 53.8 & 51.4 & 116.7 & 103.3 & 126.6 & 70.5 & 49.1 & 46.4 & 39 & 38.2 & 801.8 & 8.006 & .880 \\
\hline Schoo & 9.2 & 6.6 & 7.0 & 6.1 & 8.9 & 7.8 & 8.7 & 7.3 & 6.4 & 5. & 5. & 5.9 & 85.5 & .381 & .093 \\
\hline Simpsc & 37.2 & 26.9 & 29.4 & 30.9 & 32.1 & 34.7 & 24.6 & 31.9 & 29.2 & 30. & 26.8 & 31.2 & e) 365.7 & $\cdots$ &.- \\
\hline State Hosp., Kalamazoo & 14.2 & 8.6 & 14.4 & 8.3 & 8.1 & 6.3 & 7.0 & 6.6 & 5.9 & 8.0 & 8.1 & 8.9 & 104.4 & .563 & .155 \\
\hline Upjoh & 487.0 & 488.6 & 532.9 & 509.1 & 558.4 & 535.5 & 537.0 & 527.7 & 452.9 & 498.9 & 447.4 & 480.6 & $6,056.0$ & 23.999 & 10.491 \\
\hline Vicksbur & 8.7 & 10.6 & 12.6 & 9.9 & 16.9 & 12.8 & 17.9 & 10.9 & 9.7 & 8.6 & 8.1 & 8.2 & 134.9 & 2.267 & .084 \\
\hline
\end{tabular}


TABLE 2. REPORTED GROUND-WATER PUMPAGE, IN 1977. (IN MILLIONS OF GALLONS).continUED

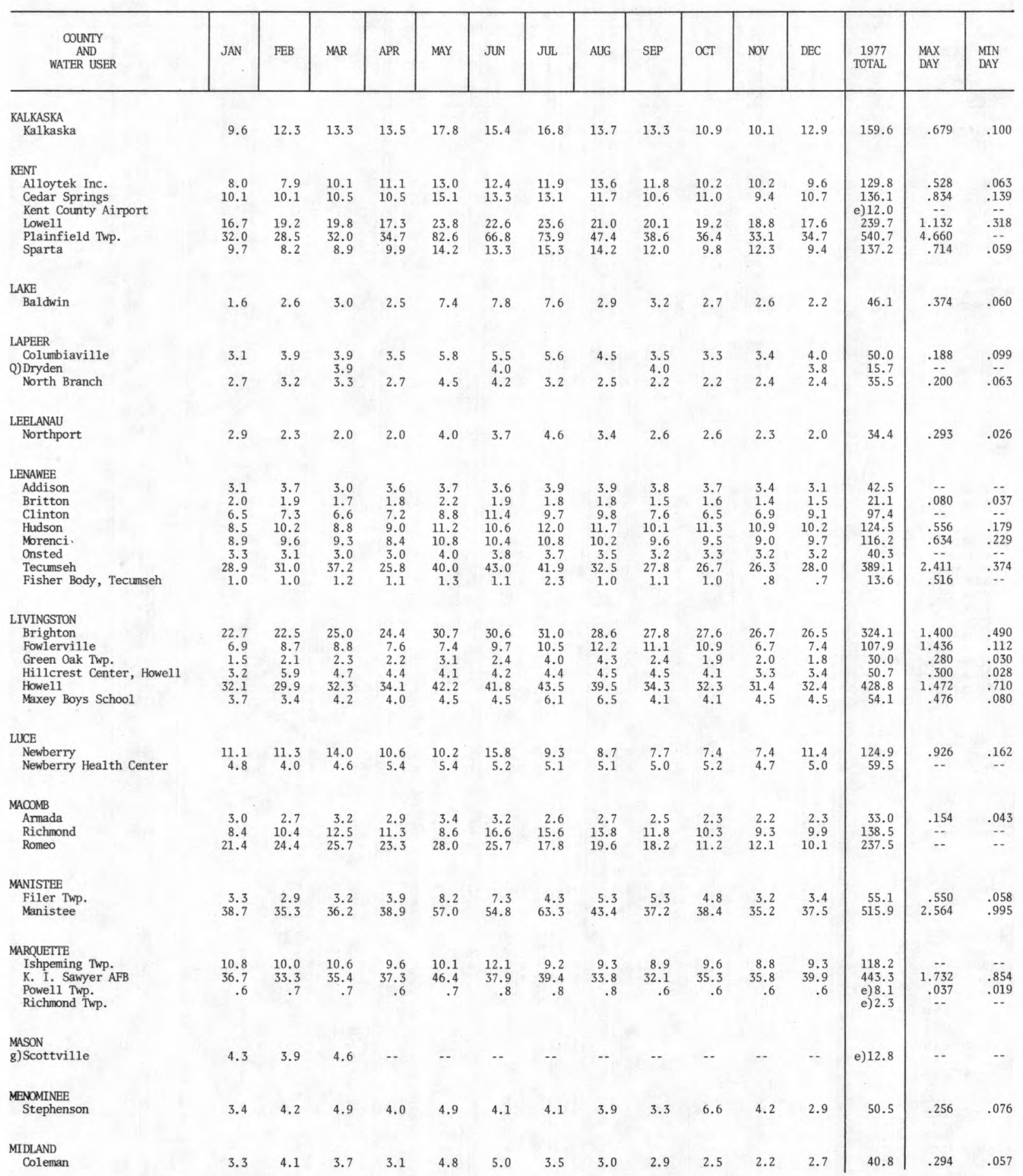


TABLE 2. REPORTED GROUND-WATER PUMPAGE, IN 1977. (IN MILLIONS OF GALLONS)-CONTINUED

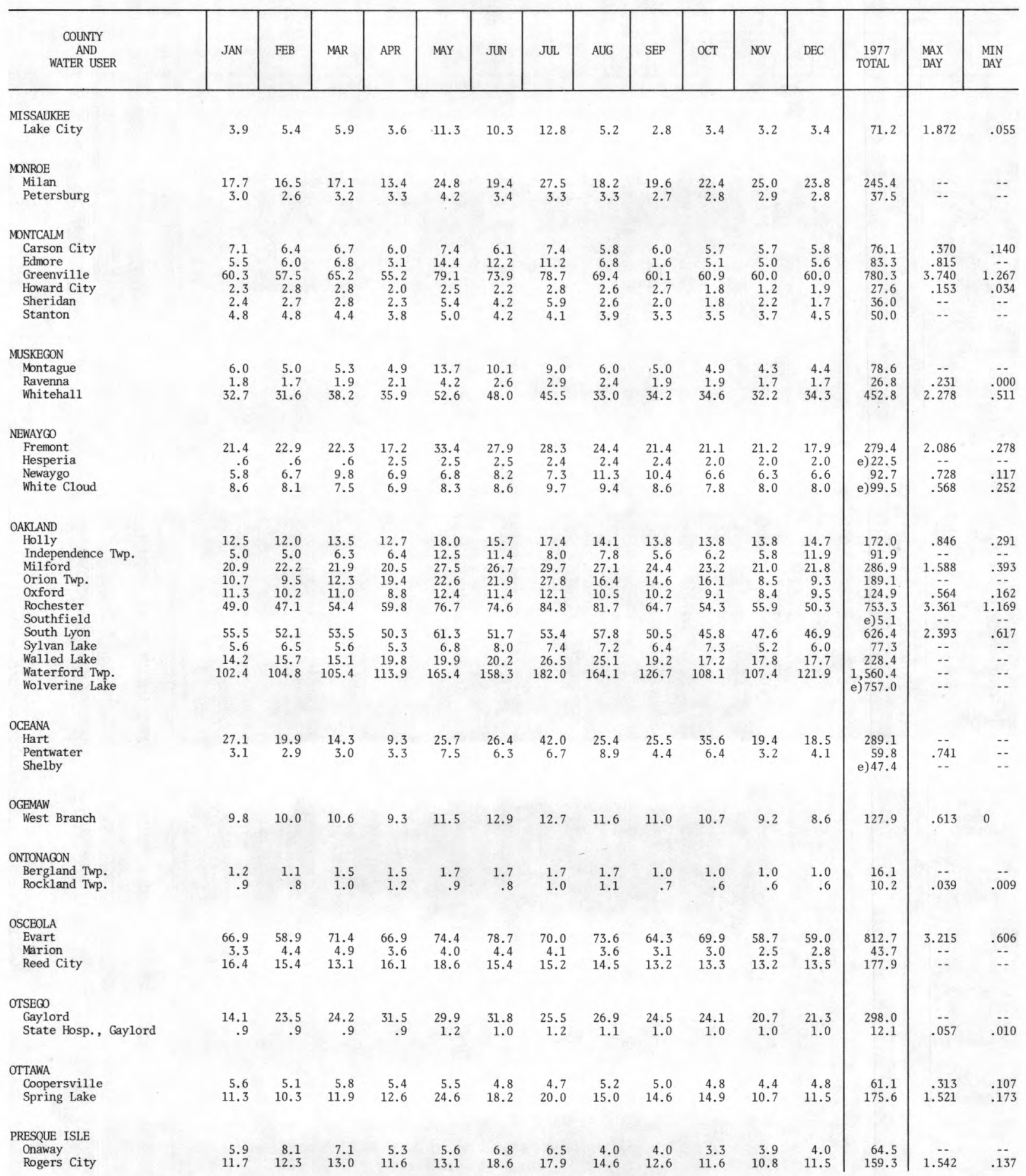


TABLE 2. REPORTED GROUND-WATER PUMPAGE, IN 1977. (IN MILLIONS OF GALLONS)-continued

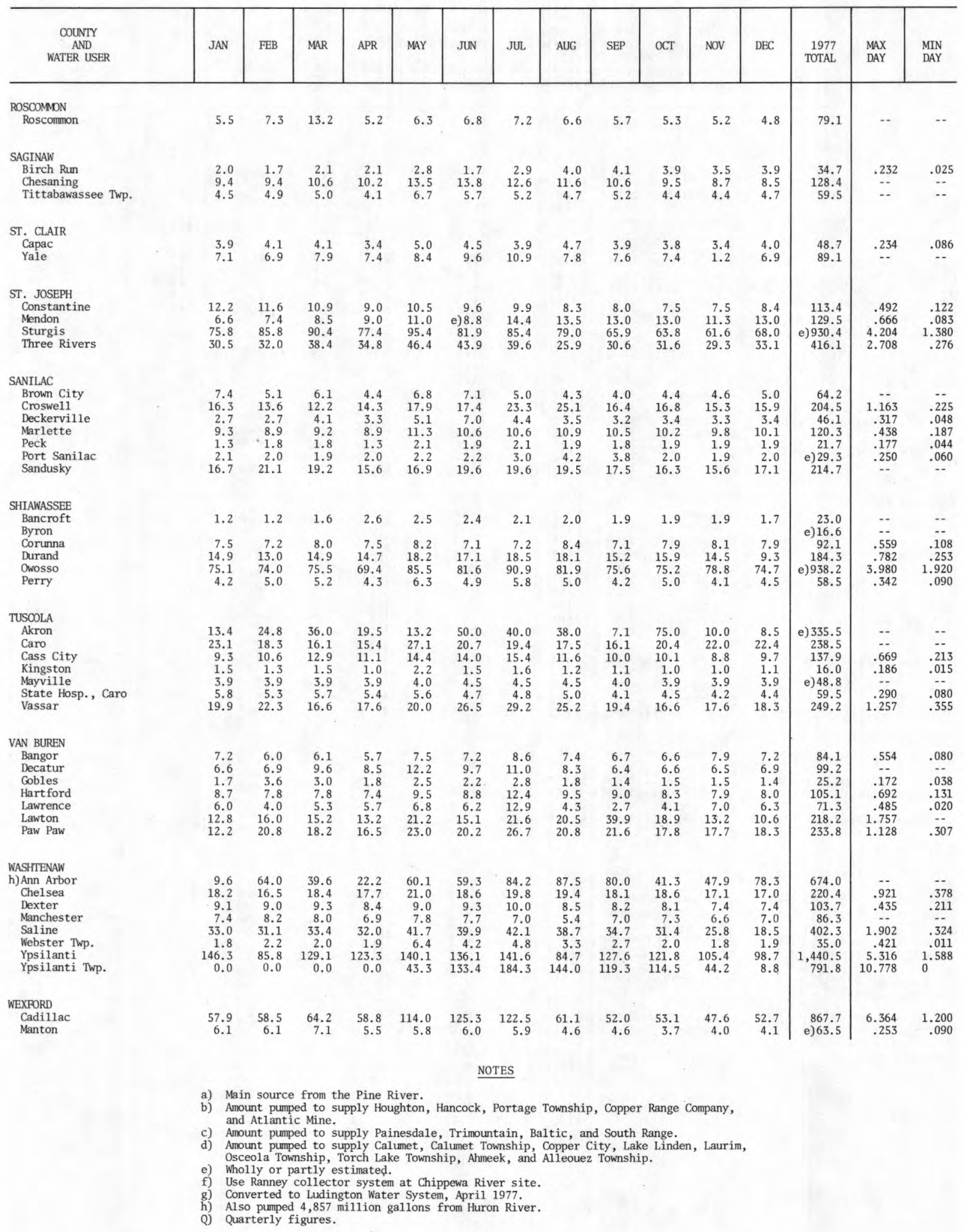


LOCAL IDENTIFIER: For explanation of well numbers, see text under - Introduction "we11 numbering system," also includes abbreviated spelling of county name. GEOLOGIC UNIT: See end of table.

UNITS: Units are reported in JTU = Jackson Turbidity Units; MG/L = Milligrams per Liter; UG/L = Micrograms per Liter.

\begin{tabular}{|c|c|c|c|c|c|c|c|}
\hline $46 N$ & $20 w$ & $0 \triangle C A C C 01$ & ALGER & $77-07-25$ & $1125 D G V$ & 60 & 8.0 \\
\hline $32 N$ & $06 E$ & $23000 A 01$ & ALPENA & $77-09-28$ & IIISAND & 88 & 9.0 \\
\hline $31 \mathrm{~N}$ & $06 w$ & 32008001 & ANTRIM & $77-07-28$ & $11250 G V$ & 34 & 8.0 \\
\hline $49 \mathrm{~N}$ & $34 w$ & $14 \mathrm{ADDOO01}$ & BARAGA & $77-08-23$ & 112 SAND & 139 & 14.5 \\
\hline $17 N$ & $04 \mathrm{E}$ & $221) C A A 01$ & BAY & $75-06-24$ & $324 \mathrm{SGNW}$ & 110 & 9.0 \\
\hline & & & & $76-06-24$ & & 110 & 9.0 \\
\hline 26iv & $14 w$ & $13 A A B E 01$ & BENZIE & $77-07-27$ & 112SAND & 214 & 9.0 \\
\hline $26 \mathrm{~N}$ & $14 w$ & 13ABВAA01 & BENZIE & $77-07-27$ & 11 2SAND & 50 & 9.0 \\
\hline $06 \mathrm{~S}$ & $06 \mathrm{~W}$ & $18 \mathrm{CCCO} 01$ & BRANCH & $\begin{array}{l}75-06-11 \\
76-06-16\end{array}$ & $1120 \mathrm{TSH}$ & $\begin{array}{l}56 \\
56\end{array}$ & $\begin{array}{l}13.5 \\
11.0\end{array}$ \\
\hline $01 \mathrm{~s}$ & $07 w$ & $32 \mathrm{RDCCO} 01$ & CALHUN & $\begin{array}{l}75-06-11 \\
76-06-17\end{array}$ & 337MRSL & $\begin{array}{l}95 \\
95\end{array}$ & $\begin{array}{l}11.0 \\
10.5\end{array}$ \\
\hline $33 N$ & $01 \mathrm{~W}$ & $26 D A B B 01$ & CHBYGN & $\begin{array}{l}75-06-24 \\
76-06-23\end{array}$ & $1125 A N D$ & $\begin{array}{l}164 \\
164\end{array}$ & $\begin{array}{l}8.5 \\
8.5\end{array}$ \\
\hline $37 \mathrm{~N}$ & $03 w$ & 33АDBНO1 & CHBYGN & $77-07-29$ & $112 S A N D$ & 83 & 9.5 \\
\hline $46 \mathrm{~N}$ & $04 \mathrm{~W}$ & 240ADA01 & CHIPWA & $\begin{array}{l}75-09-25 \\
76-06-08\end{array}$ & $1120 \mathrm{TSH}$ & $\begin{array}{l}54 \\
54\end{array}$ & $\begin{array}{l}8.5 \\
9.0\end{array}$ \\
\hline & & & & $\begin{array}{l}77-09-26 \\
77-09-20\end{array}$ & & 54 & 9.0 \\
\hline $\begin{array}{l}50 N \\
19 N\end{array}$ & $04 \mathrm{~W}$ & $\begin{array}{l}\text { 21CCAA01 } \\
21 D B C C 01\end{array}$ & $\begin{array}{l}\text { CLARE } \\
\text { CLAPWA }\end{array}$ & $\begin{array}{l}77-09-20 \\
77-09-27\end{array}$ & 112 SANO & 60 & 9.0 \\
\hline & & & & & 112GLCL & 208 & 9.5 \\
\hline $\begin{array}{l}05 N \\
25 N\end{array}$ & $02 N$ & З1СВВАО1 & CLINTN & $76-06-07$ & $324 \mathrm{SGNW}$ & 195 & 11.5 \\
\hline $25 \mathrm{~N}$ & $01 \mathrm{w}$ & $1500 C D 01$ & CRWFRO & $76-06-23$ & $112 G \mathrm{LLL}$ & 56 & 9.0 \\
\hline $38 \mathrm{~N}$ & $19 \mathrm{~W}$ & 04HCBA01 & DEL TA & $77-08-10$ & 3558BLF & 160 & 9.5 \\
\hline $41 \mathrm{~N}$ & $19 w$ & ¿UADBE01 & DEL.TA & $\begin{array}{l}76-11-23 \\
77-07-27\end{array}$ & $372 L K S P$ & $\begin{array}{l}1160 \\
1160\end{array}$ & $\begin{array}{l}13.0 \\
13.0\end{array}$ \\
\hline $43 N$ & $28 w$ & 32ADABO 1 & DCKNSN & $\begin{array}{l}75-09-30 \\
77-08-04\end{array}$ & 112 SAND & $\begin{array}{l}31 \\
31\end{array}$ & $\begin{array}{l}7.5 \\
7.0\end{array}$ \\
\hline $04 N$ & $04 \mathrm{~W}$ & $11 \mathrm{ABAAO1}$ & EATON & $\begin{array}{l}75-05-23 \\
76-06-03\end{array}$ & 324 SGNW & $\begin{array}{l}350 \\
350\end{array}$ & 12.5 \\
\hline $35 N$ & $04 \mathrm{~W}$ & $1 \triangle B C A C 01$ & EMMET & $77-07-28$ & 112 SANO & 330 & 7.5 \\
\hline $35 N$ & $04 \mathrm{~W}$ & $18 \mathrm{ACACO2}$ & EMME T & $77-07-28$ & 341 TRVR & 471 & 8.0 \\
\hline $06 \mathrm{~N}$ & $07 E$ & $091) \mathrm{CCCO}_{1}$ & GENESE & $\begin{array}{l}75-06-23 \\
76-06-30\end{array}$ & $324 \mathrm{SGNW}$ & $\begin{array}{l}385 \\
385\end{array}$ & $\begin{array}{l}10.5 \\
10.0\end{array}$ \\
\hline $20 N$ & $02 F$ & 32RBBDOI & GL.ADWN & $77-09-29$ & 324 SGNW & 139 & 9.0 \\
\hline $44 N$ & $42 w$ & OOBDDA01 & GOGEBC & $77-08-16$ & $1125 D G V$ & 100 & 8.0 \\
\hline $26 N$ & $09 w$ & $14 \mathrm{ABAAO} 1$ & GD TRV & $\begin{array}{l}76-06-22 \\
77-07-27\end{array}$ & $112 S A N D$ & $\begin{array}{l}80 \\
80\end{array}$ & $\begin{array}{l}7.5 \\
---\end{array}$ \\
\hline $12 \mathrm{iv}$ & $03 w$ & $240 \mathrm{ABB} 01$ & GRT IOT & $76-06-25$ & $1120 \mathrm{TSH}$ & 216 & 10.0 \\
\hline 075 & $02 w$ & $1080 D 001$ & HILSDL & $76-06-16$ & 112SAND & 20 & 8.5 \\
\hline $47 \mathrm{~N}$ & $37 w$ & $04 \mathrm{BBBA01}$ & HOGHTN & $77-08-23$ & $374 \mathrm{JCBV}$ & 150 & 7.5 \\
\hline $52 N$ & $36 w$ & 22CBDDOI & HOGHTN & $77-08-22$ & 112 SAND & 182 & $14 \cdot 0$ \\
\hline $02 N$ & $01 \mathrm{~W}$ & $05 В С А В 01$ & INGHAM & $\begin{array}{l}75-05-20 \\
76-06-09\end{array}$ & $324 \mathrm{SGNW}$ & $\begin{array}{l}210 \\
210\end{array}$ & $\begin{array}{l}12.0 \\
10.0\end{array}$ \\
\hline $04 N$ & $01 E$ & $21 C O D A 01$ & INGHAM & $76-06-08$ & $324 \mathrm{SGNW}$ & 265 & 11.5 \\
\hline $44 \mathrm{iv}$ & $31 w$ & 35ABCBOI & IRON & $77-08-03$ & $112 S A N D$ & 159 & $8 \cdot 0$ \\
\hline $03 \mathrm{~S}$ & $01 w$ & 0250BA01 & JACKSN & $76-06-09$ & $324 \mathrm{SGNW}$ & 400 & $12 \cdot 0$ \\
\hline $03 \mathrm{~S}$ & $01 w$ & $11 \mathrm{AADD} 02$ & JACKSN & $\begin{array}{l}75-05-27 \\
76-06-09\end{array}$ & $112015 H$ & $\begin{array}{l}36 \\
36\end{array}$ & \\
\hline $04 \mathrm{~S}$ & $11 w$ & $03 C D D A 01$ & KALMZO & $\begin{array}{l}75-06-10 \\
76-06-17\end{array}$ & $112015 H$ & $\begin{array}{l}190 \\
190\end{array}$ & $\begin{array}{l}12.5 \\
10.0\end{array}$ \\
\hline $05 N$ & $12 W$ & 04DCCDOI & KENT & $76-06-21$ & $112 \mathrm{GRVL}$ & 86 & 10.0 \\
\hline $06 \mathrm{~N}$ & $12 W$ & $27 \sqcup B B A 01$ & KENT & $75-06-26$ & 337MRSL & 265 & 10.0 \\
\hline $17 \mathrm{~N}$ & $13 w$ & 04ADAA01 & LAKE & $\begin{array}{l}76-06-21 \\
76-06-22\end{array}$ & $112 \mathrm{GLCL}$ & $\begin{array}{r}265 \\
83\end{array}$ & $\begin{array}{l}9.5 \\
9.5\end{array}$ \\
\hline
\end{tabular}

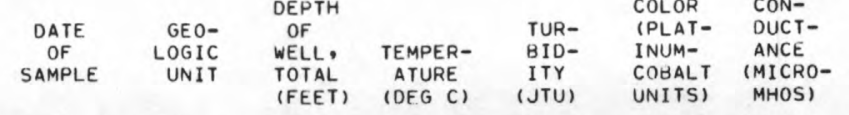

$\begin{array}{ccc} & \text { CARBON } \\ \text { DIOXIDE } & \text { ALKA- } \\ \text { DIS- } & \text { LINITY } \\ \text { POL } & \text { SOLVE } & \text { (MG/L } \\ \text { (UNITS) AS COL) } & \text { CACOB) }\end{array}$

LOCAL
IDENT-
I-
FIER

1
4
2
1
7

$\begin{array}{r}5 \\ 1 \\ 40 \\ 0 \\ 11 \\ \hline-\end{array}$

228

8.0
7.5
7.4
8.0
7.8

$\begin{array}{cc}2.4 & 123 \\ 13 & 220 \\ 15 & 197 \\ 2.1 & 107 \\ 4.4 & 141\end{array}$

196
3250

3440
559

7.6

141

570

7.7

7.6

7.2
7.2
1.7

148

590

7.5

17

200

600

700

454

7.6
7.6
7.9

13
14

14

5.6
7.4

7.8

5.2

271

293

7.6

40
37
37
160
400

$\because 4$

6.6
6.4

6.4
7.4
7.8

8.3
5.6

261

287

230
238

\begin{tabular}{l}
167 \\
\hline
\end{tabular}

7.4
7.7

790

290

584

800
905

7.7
7.2
7.4

7.4
7.8

27

6.1

39
13

8.2

300

306

774

625
323

7.9
7.6
7.6
7.9

$\begin{array}{ll}1.9 & 153 \\ 4.2 & 170 \\ 15 & 312 \\ 16 & 320\end{array}$

16
4.5

-

72

190

350

485

490

7.6

9.2

8.0

$\begin{array}{ll}9.2 & 187\end{array}$

7.8

8.4
2.8
2.4

153
170
312
320
182
187
256
271
91
156

1544
254

8.1

250
1010
790

7.8
7.9
8.9

4.1
3.0
25

8.9
7.4

7.6

3.9

$\begin{array}{ll}7.9 & 3 . \\ 7.7 & 18\end{array}$

$\begin{array}{rrr}1 & 0 & 220 \\ 60 & 80 & 1200\end{array}$

$--$

40

1280
420

6.7
7.0
7.5

18
188
48

48
16

$\begin{array}{rr}45 & 1 \\ -8 & --\end{array}$

3500

1040
1180
475

$--$

25
25

475
470

7.4
8.3

7.7

2.6
9.9

344

156

166

$\begin{array}{rr}3 & 8 \\ 4 & 3 \\ -5 & --\end{array}$

400
500
590
350

7.6
7.6
7.4
8.0

$\begin{array}{rr}7.6 & 156 \\ 1.1 & 23 \\ 7.0 & 90 \\ 2.2 & 115\end{array}$


TABLE 3. WATER-QUAlity DATA. (COntinued)

\begin{tabular}{|c|c|c|c|c|c|c|c|c|c|c|c|c|c|}
\hline & & $\begin{array}{l}\text { LOCAL } \\
\text { IDENT- } \\
\text { I- } \\
\text { FIER }\end{array}$ & & $\begin{array}{c}\text { DATE } \\
\text { OF } \\
\text { SAMPLE }\end{array}$ & $\begin{array}{l}\text { GEO- } \\
\text { LOGIC } \\
\text { UNIT }\end{array}$ & $\begin{array}{l}\text { DEPTH } \\
\text { OF } \\
\text { WELL, } \\
\text { TOTAL } \\
\text { (FEET) }\end{array}$ & $\begin{array}{l}\text { TEMPER- } \\
\text { ATURE } \\
\text { (DEG C) }\end{array}$ & $\begin{array}{l}\text { TUR- } \\
\text { BID- } \\
\text { ITY } \\
\text { (JTU) }\end{array}$ & $\begin{array}{l}\text { COLOR } \\
\text { (PLAT- } \\
\text { INUM- } \\
\text { COBALT } \\
\text { UNITS) }\end{array}$ & $\begin{array}{l}\text { SPE- } \\
\text { CIFIC } \\
\text { CON- } \\
\text { DUCT- } \\
\text { ANCE } \\
\text { (MICRO- } \\
\text { MHOS) }\end{array}$ & $\begin{array}{c}\text { PH } \\
\text { (UNITS) }\end{array}$ & $\begin{array}{l}\text { CARBON } \\
\text { DIOXIDE } \\
\text { OIS- } \\
\text { SOLVED } \\
\text { (MG/L } \\
\text { AS COZ) }\end{array}$ & $\begin{array}{l}\text { ALKA- } \\
\text { LINITY } \\
\text { (MG/L } \\
\text { AS } \\
\text { CACO3) }\end{array}$ \\
\hline $06 \mathrm{~S}$ & $\begin{array}{l}06 E \\
12 W\end{array}$ & $\begin{array}{l}\text { O8ODBA01 } \\
1 \text { 130BAB01 } \\
\text { O1BACB01 }\end{array}$ & $\begin{array}{l}\text { LENAWE } \\
\text { LVNGTN } \\
\text { LUCE }\end{array}$ & $\begin{array}{l}75-05-27 \\
76-06-14 \\
75-05-22 \\
76-06-10 \\
77-07-26\end{array}$ & $\begin{array}{l}\text { 1120TSH } \\
1120 \mathrm{TSH} \\
112 \mathrm{SDGV}\end{array}$ & $\begin{array}{l}81 \\
81 \\
29 \\
29 \\
44\end{array}$ & $\begin{array}{r}14.0 \\
11.00 \\
18.5 \\
14.5 \\
8.0\end{array}$ & $\begin{array}{r}15 \\
-\frac{2}{1} \\
--\end{array}$ & $\begin{array}{r}-7 \\
-\frac{7}{5} \\
--\end{array}$ & $\begin{array}{l}768 \\
840 \\
748 \\
690 \\
182\end{array}$ & $\begin{array}{l}7.9 \\
7.5 \\
7.5 \\
7.4 \\
8.0\end{array}$ & $\begin{array}{l}6.0 \\
17 \\
19 \\
25 \\
1.6\end{array}$ & $\begin{array}{r}243 \\
279 \\
312 \\
320 \\
80\end{array}$ \\
\hline $\begin{array}{l}42 N \\
43 N \\
44 N\end{array}$ & $\begin{array}{l}02 W \\
09 w \\
26 W\end{array}$ & $\begin{array}{l}\text { 07AABBO1 } \\
\text { 13DDDA01 } \\
280 A B B 01\end{array}$ & $\begin{array}{l}\text { MACKNC } \\
\text { MACKNC } \\
\text { MARQTE }\end{array}$ & $\begin{array}{l}76-06-09 \\
77-09-27 \\
77-09-22 \\
76-11-22 \\
77-09-29\end{array}$ & $\begin{array}{l}355 \mathrm{MNSQ} \\
355 \mathrm{ENGD} \\
112 \text { SAND }\end{array}$ & $\begin{array}{r}102 \\
102 \\
54 \\
28 \\
28\end{array}$ & $\begin{array}{l}8.0 \\
8.0 \\
7.0 \\
7.0 \\
7.0\end{array}$ & $\begin{array}{r}1 \\
1 \\
70 \\
2 \\
1\end{array}$ & $\begin{array}{r}1 \\
2 \\
45 \\
5 \\
2\end{array}$ & $\begin{array}{l}380 \\
460 \\
369 \\
380 \\
413\end{array}$ & $\begin{array}{l}7.5 \\
7.3 \\
7.7 \\
7.6 \\
7.8\end{array}$ & $\begin{array}{l}14 \\
26 \\
6.3 \\
11 \\
6.9\end{array}$ & $\begin{array}{l}224 \\
260 \\
161 \\
220 \\
220\end{array}$ \\
\hline $47 \mathrm{~N}$ & $26 \mathrm{~W}$ & 36880801 & MARQTE & $\begin{array}{l}76-06-01 \\
76-11-03 \\
77-07-18 \\
75-09-23 \\
76-06-01\end{array}$ & 1120 TSH & $\begin{array}{l}56 \\
56 \\
56 \\
75 \\
75\end{array}$ & $\begin{array}{l}7.5 \\
8.0 \\
8.0 \\
7.0 \\
7.5\end{array}$ & $\begin{array}{r}1 \\
4 \\
1 \\
3 \\
--\end{array}$ & $\begin{array}{r}70 \\
80 \\
100 \\
150 \\
--\end{array}$ & $\begin{array}{l}198 \\
220 \\
213 \\
200 \\
185\end{array}$ & $\begin{array}{l}8.0 \\
8.1 \\
7.8 \\
7.9 \\
7.4\end{array}$ & $\begin{array}{l}2.1 \\
1.8 \\
4.1 \\
2.3 \\
-.-\end{array}$ & $\begin{array}{r}110 \\
118 \\
131 \\
95\end{array}$ \\
\hline $\begin{array}{l}47 \mathrm{~N} \\
18 \mathrm{~N} \\
33 \mathrm{~N}\end{array}$ & $\begin{array}{l}29 w \\
17 w \\
27 w\end{array}$ & $\begin{array}{l}02 \triangle D D A 01 \\
14 C A B A 01 \\
35 B B C B 01\end{array}$ & $\begin{array}{l}\text { MARQTE } \\
\text { MASON } \\
\text { MENOME }\end{array}$ & $\begin{array}{l}76-11-04 \\
77-07-20 \\
77-07-20 \\
77-07-26 \\
77-06-30\end{array}$ & $\begin{array}{l}\text { 112SDGV } \\
112 S A N D \\
\text { 36SBKRV }\end{array}$ & $\begin{array}{r}75 \\
75 \\
15 \\
103 \\
147\end{array}$ & $\begin{array}{r}7.5 \\
8.0 \\
11.0 \\
10.5 \\
9.0\end{array}$ & $\begin{array}{r}8 \\
15 \\
3 \\
1 \\
3\end{array}$ & $\begin{array}{r}150 \\
140 \\
200 \\
10 \\
5\end{array}$ & $\begin{array}{l}200 \\
190 \\
132 \\
324 \\
900\end{array}$ & $\begin{array}{l}7.6 \\
7.5 \\
7.2 \\
8.0 \\
7.3\end{array}$ & $\begin{array}{l}4.5 \\
6.1 \\
5.8 \\
3.5 \\
39\end{array}$ & $\begin{array}{r}92 \\
98 \\
47 \\
180 \\
400\end{array}$ \\
\hline $\begin{array}{l}23 N \\
31 N \\
11 N\end{array}$ & $\begin{array}{l}26 W \\
05 W \\
04 E \\
15 W\end{array}$ & $\begin{array}{l}190 A D A 01 \\
35 C A B A 01 \\
05 C B D B 01 \\
34 A D O D 01\end{array}$ & $\begin{array}{l}\text { MENOME } \\
\text { MSAUKE } \\
\text { MNTMRY } \\
\text { MUSKGN }\end{array}$ & $\begin{array}{l}76-11-24 \\
77-06-29 \\
77-07-29 \\
77-09-28 \\
76-06-28\end{array}$ & $\begin{array}{l}365 \mathrm{TBRV} \\
112 \mathrm{GLCL} \\
112 \mathrm{GRVL} \\
112 \mathrm{SAND}\end{array}$ & $\begin{array}{l}17 \\
17 \\
54 \\
37 \\
31\end{array}$ & $\begin{array}{r}10.0 \\
8.5 \\
9.0 \\
8.0 \\
9.5\end{array}$ & $\begin{array}{r}3 \\
4 \\
3 \\
0 \\
40\end{array}$ & $\begin{array}{r}10 \\
5 \\
10 \\
3 \\
7\end{array}$ & $\begin{array}{r}500 \\
570 \\
353 \\
289 \\
1790\end{array}$ & $\begin{array}{l}7.5 \\
7.4 \\
7.8 \\
7.8 \\
7.3\end{array}$ & $\begin{array}{l}16 \\
23 \\
6.3 \\
4.8 \\
37\end{array}$ & $\begin{array}{l}255 \\
300 \\
203 \\
150 \\
377\end{array}$ \\
\hline $\begin{array}{l}02 N \\
03 N\end{array}$ & $\begin{array}{l}08 E \\
08 E \\
01 E\end{array}$ & $\begin{array}{l}18 U B A D 01 \\
030 B A B 01 \\
\text { OZAAAA02 }\end{array}$ & $\begin{array}{l}\text { OAKLND } \\
\text { OAKLND } \\
\text { OGEMAW }\end{array}$ & $\begin{array}{l}75-05-22 \\
76-06-10 \\
75-05-22 \\
76-06-10 \\
76-06-24\end{array}$ & $\begin{array}{l}1120 \text { TSH } \\
1120 \text { TSH } \\
112 \text { SAND }\end{array}$ & $\begin{array}{r}45 \\
45 \\
163 \\
163 \\
20\end{array}$ & $\begin{array}{r}10.0 \\
9.5 \\
9.5 \\
9.5 \\
7.0\end{array}$ & $\begin{array}{l}15 \\
-- \\
15 \\
--\end{array}$ & $\begin{array}{r}0 \\
-- \\
-- \\
--\end{array}$ & $\begin{array}{l}658 \\
665 \\
522 \\
510 \\
370\end{array}$ & $\begin{array}{l}7.7 \\
6.8 \\
8.3 \\
6.8 \\
7.6\end{array}$ & $\begin{array}{l}13 \\
94 \\
2.6 \\
84 \\
8.4\end{array}$ & $\begin{array}{l}323 \\
303 \\
261 \\
271 \\
172\end{array}$ \\
\hline $\begin{array}{l}46 \mathrm{~N} \\
48 \mathrm{~N} \\
51 \mathrm{~N}\end{array}$ & $\begin{array}{l}38 w \\
43 w \\
41 w\end{array}$ & $\begin{array}{l}30 \triangle 00001 \\
13 C D D A 01 \\
08 B D B C 01\end{array}$ & $\begin{array}{l}\text { ONTNGN } \\
\text { ONTNGN } \\
\text { ONTNGN }\end{array}$ & $\begin{array}{l}77-09-29 \\
76-06-14 \\
77-08-18 \\
75-09-22 \\
76-00-15\end{array}$ & $\begin{array}{l}112 S D G V \\
374 \mathrm{JCBV} \\
400 \mathrm{FRED}\end{array}$ & $\begin{array}{r}20 \\
65 \\
60 \\
100 \\
100\end{array}$ & $\begin{array}{r}10.5 \\
8.5 \\
9.0 \\
8.0 \\
9.0\end{array}$ & $\begin{array}{r}4 \\
1 \\
1 \\
95 \\
--\end{array}$ & $\begin{array}{r}2 \\
1 \\
10 \\
15 \\
--\end{array}$ & $\begin{array}{l}345 \\
156 \\
545 \\
540 \\
415\end{array}$ & $\begin{array}{l}7.7 \\
8.0 \\
7.2 \\
8.0 \\
7.9\end{array}$ & $\begin{array}{r}6.4 \\
1.6 \\
25 \\
3.1 \\
--\end{array}$ & $\begin{array}{r}170 \\
84 \\
205 \\
159\end{array}$ \\
\hline $\begin{array}{l}31 \mathrm{~N} \\
33 \mathrm{~N}\end{array}$ & $\begin{array}{l}03 W \\
06 E \\
02 W\end{array}$ & $\begin{array}{l}34 \mathrm{ACDDO} 1 \\
08 B \mathrm{BBH01}\end{array}$ & $\begin{array}{l}\text { OTSEGO } \\
\text { PRSQ I } \\
\text { ROSCMN }\end{array}$ & $\begin{array}{l}77-07-28 \\
75-06-24 \\
76-06-23 \\
75-06-25 \\
76-06-24\end{array}$ & $\begin{array}{l}\text { 112SAND } \\
341 \text { TRVR } \\
1120 \text { TSH }\end{array}$ & $\begin{array}{r}199 \\
61 \\
61 \\
14 \\
14\end{array}$ & $\begin{array}{r}8.5 \\
8.5 \\
8.5 \\
13.5 \\
7.5\end{array}$ & $\begin{array}{l}1 \\
15 \\
-25 \\
--\end{array}$ & $\begin{array}{l}5 \\
11 \\
65 \\
65 \\
--\end{array}$ & $\begin{array}{l}456 \\
593 \\
640 \\
320 \\
330\end{array}$ & $\begin{array}{l}7.6 \\
7.6 \\
7.2 \\
8.1 \\
7.8\end{array}$ & $\begin{array}{l}9.7 \\
12 \\
34 \\
2.8 \\
5.8\end{array}$ & $\begin{array}{l}198 \\
239 \\
279 \\
179 \\
189\end{array}$ \\
\hline $12 \mathrm{~N}$ & $13 E$ & 33000001 & SANLAC & $\begin{array}{l}75-06-23 \\
76-06-29\end{array}$ & 337 MRSL & $\begin{array}{l}150 \\
150\end{array}$ & $\begin{array}{l}12.0 \\
10.0\end{array}$ & -3 & -6 & $\begin{array}{l}615 \\
650 \\
380\end{array}$ & $\begin{array}{l}7.7 \\
7.4\end{array}$ & $\begin{array}{l}15 \\
31 \\
5.0\end{array}$ & $\begin{array}{l}374 \\
394 \\
205\end{array}$ \\
\hline $\begin{array}{l}13 N \\
45 N\end{array}$ & $\begin{array}{l}13 E \\
13 W\end{array}$ & $\begin{array}{l}12 A D A A 01 \\
16 C C C B 01\end{array}$ & $\begin{array}{l}\text { SANLAC } \\
\text { SCHCFT }\end{array}$ & $\begin{array}{l}76-06-29 \\
75-09-24 \\
76-06-07\end{array}$ & $\begin{array}{l}\text { 337MRSL } \\
\text { 362RCMD }\end{array}$ & $\begin{array}{l}130 \\
154 \\
154\end{array}$ & $\begin{array}{r}9.5 \\
15.0 \\
11.0\end{array}$ & $\begin{array}{r}55 \\
3 \\
--\end{array}$ & $\begin{array}{r}1 \\
0 \\
--\end{array}$ & $\begin{array}{l}380 \\
800 \\
354\end{array}$ & $\begin{array}{l}7.9 \\
7.6\end{array}$ & $\begin{array}{r}2.0 \\
-\therefore\end{array}$ & $\begin{array}{r}205 \\
70\end{array}$ \\
\hline $\begin{array}{l}045 \\
025\end{array}$ & $\begin{array}{l}16 \mathrm{~W} \\
16 \mathrm{~W} \\
03 \mathrm{E}\end{array}$ & $\begin{array}{l}308 B 8 B 01 \\
14 \mathrm{CDBD01} \\
090 A A B 02\end{array}$ & $\begin{array}{l}\text { SCHCFT } \\
\text { VN BUR } \\
\text { WSHTNW }\end{array}$ & $\begin{array}{l}76-06-07 \\
77-07-26 \\
76-06-17 \\
75-05-27 \\
76-06-15\end{array}$ & $\begin{array}{l}\text { 368PROC } \\
112 \text { SAND } \\
112 \text { SDGV }\end{array}$ & $\begin{array}{r}57 \\
57 \\
170 \\
48 \\
48\end{array}$ & $\begin{array}{r}7.5 \\
7.0 \\
10.5 \\
12.5 \\
10.5\end{array}$ & $\begin{array}{r}1 \\
2 \\
10\end{array}$ & $\begin{array}{r}1 \\
5 \\
1 \\
0 \\
--\end{array}$ & $\begin{array}{l}292 \\
295 \\
450 \\
253 \\
255\end{array}$ & $\begin{array}{l}7.8 \\
7.6 \\
7.6 \\
8.1 \\
8.0\end{array}$ & $\begin{array}{r}5.0 \\
7.6 \\
11 \\
1.6 \\
2.2\end{array}$ & $\begin{array}{l}161 \\
156 \\
230 \\
100 \\
115\end{array}$ \\
\hline 235 & $\begin{array}{l}06 E \\
09 W\end{array}$ & $\begin{array}{l}16 B C C D O 1 \\
04 A B B C 01\end{array}$ & $\begin{array}{l}\text { WSHTNW } \\
\text { WEXFRD }\end{array}$ & $\begin{array}{l}76-06-15 \\
76-06-22\end{array}$ & $\begin{array}{l}112 \mathrm{GLCL} \\
112 \mathrm{GLCL}\end{array}$ & $\begin{array}{r}55 \\
277\end{array}$ & $\begin{array}{r}10.0 \\
9.5\end{array}$ & 0 & $\frac{1}{1}$ & $\begin{array}{r}1340 \\
540\end{array}$ & $\begin{array}{l}7.2 \\
7.6\end{array}$ & $\begin{array}{l}44 \\
11\end{array}$ & $\begin{array}{l}361 \\
230\end{array}$ \\
\hline
\end{tabular}


TABLE 3. WATER-QUALITY DATA. (CONTINUED)

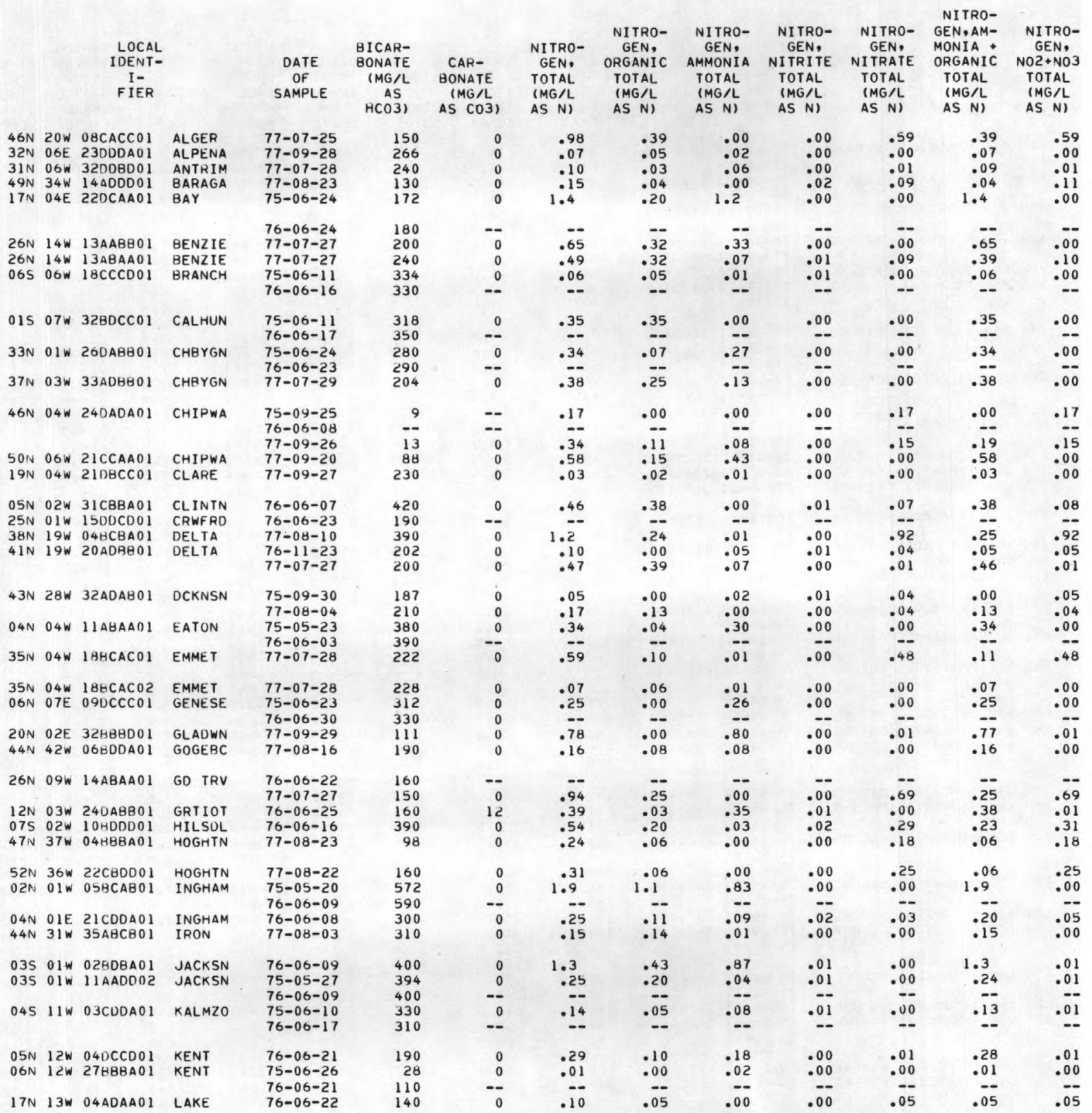


TABLE 3. WATER-QUALITY DATA. (CONTINUED)

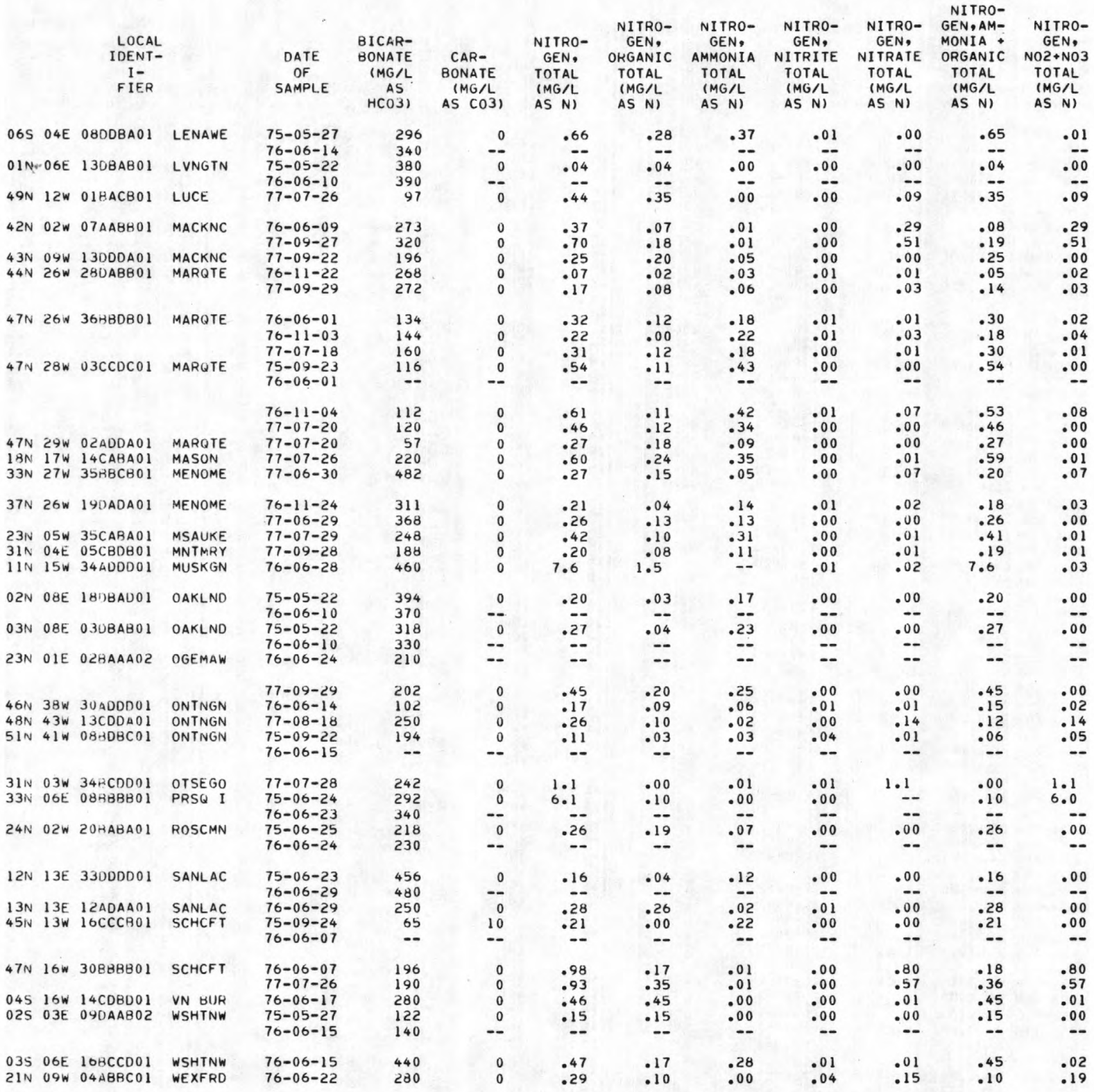


TABLE 3. WATER-QUALITY DATA. (CONTINUED)

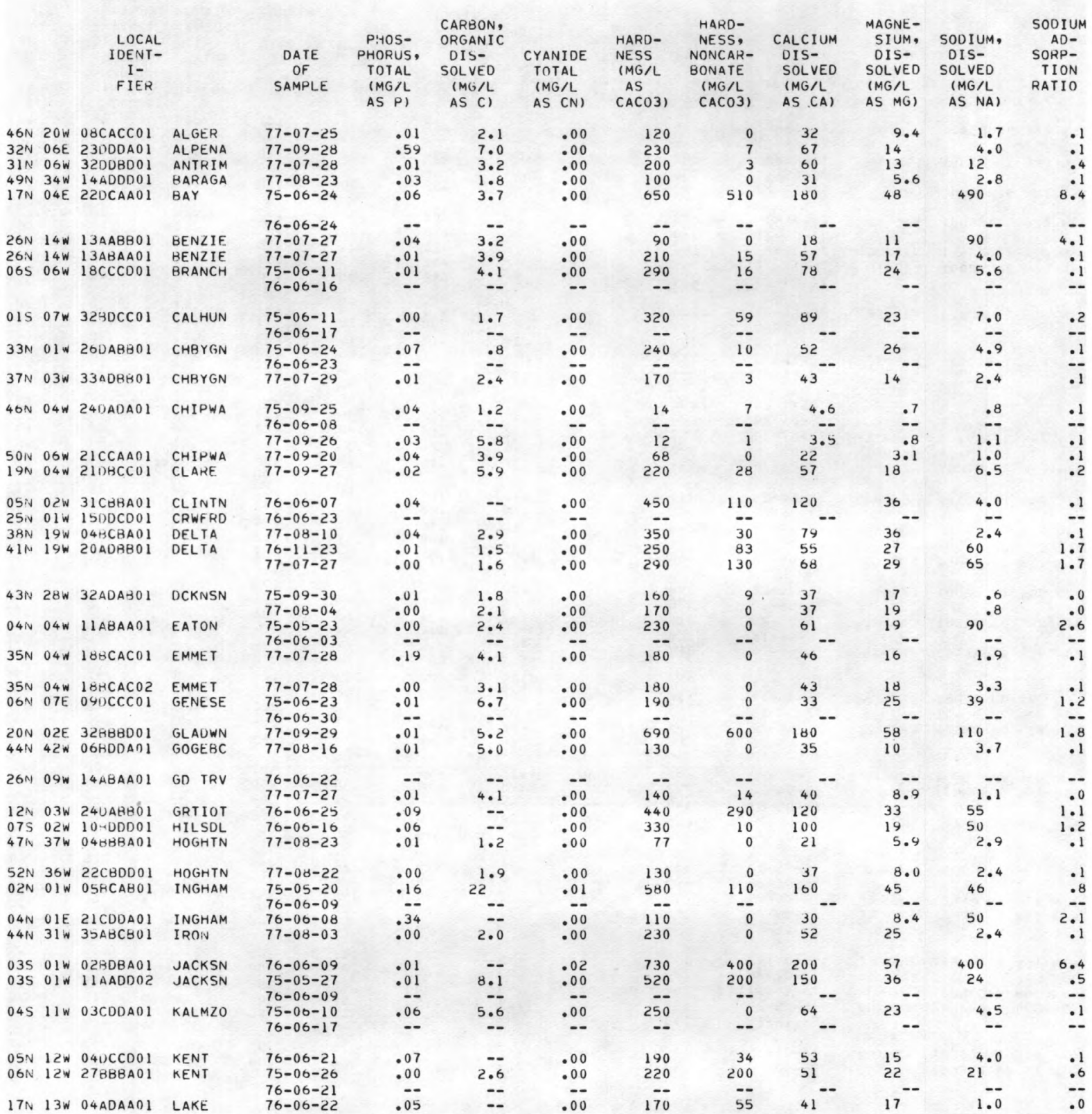


TABLE 3. WATER-QUALITY DATA. (CONTINUED)

\begin{tabular}{|c|c|c|c|c|c|c|c|c|c|c|c|c|c|}
\hline & & $\begin{array}{l}\text { LOCAL } \\
\text { IDENI- } \\
\text { I- } \\
\text { FIFR }\end{array}$ & & $\begin{array}{c}\text { DATE } \\
\text { OF } \\
\text { SAMPLE }\end{array}$ & $\begin{array}{l}\text { PHOS- } \\
\text { PHORUS, } \\
\text { TOTAL } \\
\text { (MG/L } \\
\text { AS P) }\end{array}$ & $\begin{array}{l}\text { CARBON, } \\
\text { ORGANIC } \\
\text { DIS- } \\
\text { SOLVED } \\
\text { (MG/L } \\
\text { AS C) }\end{array}$ & $\begin{array}{l}\text { CraNIDE } \\
\text { TOTAL } \\
\text { (MG/L } \\
\text { AS CN) }\end{array}$ & $\begin{array}{l}\text { HARD- } \\
\text { NESS } \\
\text { (MG/L } \\
\text { AS } \\
\text { CACO3) }\end{array}$ & $\begin{array}{l}\text { HARD- } \\
\text { NESS, } \\
\text { NONCAR- } \\
\text { BOAAE } \\
\text { (MG/L } \\
\text { CACO3) }\end{array}$ & $\begin{array}{l}\text { CALCIUM } \\
\text { OIS- } \\
\text { SOLVD } \\
\text { (MG/L } \\
\text { AS CA) }\end{array}$ & $\begin{array}{l}\text { MAGNE- } \\
\text { SIUM, } \\
\text { DIS- } \\
\text { SOLVED } \\
\text { (MGLL } \\
\text { AS MG) }\end{array}$ & $\begin{array}{l}\text { SODIUM, } \\
\text { DIS- } \\
\text { SOLED } \\
\text { (MG/L } \\
\text { AS NA) }\end{array}$ & $\begin{array}{l}\text { SODIUM } \\
\text { AD- } \\
\text { SORP- } \\
\text { TION } \\
\text { RATIO }\end{array}$ \\
\hline 65 & $04 E$ & OBDOBAOI & LENAWE & $\begin{array}{l}75-0 b-27 \\
76-05-14\end{array}$ & .03 & 8.0 & .00 & 360 & 120 & 97. & 29 & 26 & $\therefore 6$ \\
\hline $01 \mathrm{~N}$ & OSE & $1308 A B 01$ & LVNGTN & $75-05-22$ & .00 & $13^{--}$ & .00 & 400 & 88 & $110^{--}$ & $30^{--}$ & 4.5 & .1 \\
\hline $49 \mathrm{~N}$ & $12 \mathrm{w}$ & O1HACBO1 & LUCE & $\begin{array}{l}76-06-10 \\
77-07-26\end{array}$ &.$\overline{00}$ & 2.3 &.$\overline{00}$ & $\overline{89}$ & 10 & $27^{--}$ & 5.3 & 1.3 & .1 \\
\hline $42 \mathrm{~N}$ & 02w & $07 \triangle A B B O 1$ & MACKNC & $\begin{array}{l}76-05-09 \\
77-09-27\end{array}$ & $\begin{array}{r}.02 \\
.00\end{array}$ & $\overline{7.1}$ & $\begin{array}{r}.00 \\
.00\end{array}$ & $\begin{array}{l}230 \\
270\end{array}$ & $\begin{array}{l}4 \\
6\end{array}$ & $\begin{array}{l}50 \\
58\end{array}$ & $\begin{array}{l}25 \\
30\end{array}$ & $\therefore 7$ & $\because 0$ \\
\hline $4 \mathrm{~N}$ & $09 \mathrm{w}$ & $13000 A 01$ & MACKNC & $77-09-22$ & .35 & 3.2 & .00 & 190 & 29 & $\begin{array}{l}30 \\
47\end{array}$ & 18 & $\begin{array}{l}1.0 \\
2.2\end{array}$ & .1 \\
\hline $4 N$ & $26 \mathrm{w}$ & $28048 B 01$ & MARQTE & $\begin{array}{l}76-11-22 \\
77-09-29\end{array}$ & $\begin{array}{l}.01 \\
.00\end{array}$ & $\begin{array}{l}3.5 \\
6.2\end{array}$ & $\begin{array}{l}.00 \\
.00\end{array}$ & $\begin{array}{l}220 \\
230\end{array}$ & $\begin{array}{l}3 \\
6\end{array}$ & $\begin{array}{l}48 \\
49\end{array}$ & $\begin{array}{l}25 \\
26\end{array}$ & $\begin{array}{l}2.0 \\
2.2\end{array}$ & $: 1$ \\
\hline $47 \mathrm{~N}$ & $26 \mathrm{~W}$ & 368BDA01 & MARQTE & $\begin{array}{l}76-06-01 \\
76-11-03\end{array}$ & $\begin{array}{l}.03 \\
.03\end{array}$ & $16^{--}$ & $\begin{array}{l}.00 \\
.00\end{array}$ & $\begin{array}{l}110 \\
110\end{array}$ & $\frac{1}{0}$ & $\begin{array}{l}33 \\
33\end{array}$ & $\begin{array}{l}6.9 \\
6.6\end{array}$ & $\begin{array}{l}1.8 \\
1.9\end{array}$ & $\because 1$ \\
\hline $47 \mathrm{~N}$ & $28 \mathrm{w}$ & $03 \mathrm{CCDC} 01$ & MARUTE & $\begin{array}{l}77-07-18 \\
75-0 y-23\end{array}$ & $\begin{array}{l}.01 \\
.09\end{array}$ & $\begin{array}{l}4.1 \\
8.0\end{array}$ & $\begin{array}{l}.00 \\
.00\end{array}$ & $\begin{array}{l}110 \\
100\end{array}$ & $\begin{array}{l}0 \\
4\end{array}$ & $\begin{array}{l}34 \\
28\end{array}$ & $\begin{array}{l}6.9 \\
7.2\end{array}$ & $\begin{array}{l}2.3 \\
1.9\end{array}$ & $\because 1$ \\
\hline & & & & $76-06-01$ & -- & - & -- & -- & - & -- & -- & - & \\
\hline & & & & $\begin{array}{l}76-11-04 \\
77-07-20\end{array}$ & $\begin{array}{l}.07 \\
.09\end{array}$ & 11 & .00 & 89 & $\begin{array}{l}0 \\
0\end{array}$ & $\begin{array}{l}25 \\
25 \\
25\end{array}$ & 6.4 & $\begin{array}{l}1.8 \\
2.0\end{array}$ & 11 \\
\hline & $29 \mathrm{~W}$ & $02 \triangle 00 A 01$ & MARQTE & $77-0$ & : & 2.8 & .0 & 56 & 10 & 12 & 6.4 & 1.5 & $\bullet$ \\
\hline N & $17 \mathrm{~W}$ & $14 C A B A 01$ & MASON & 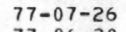 & & 4.3 & & 170 & 0 & & & & .3 \\
\hline & $27 w$ & $35 \mathrm{HBCBO1}$ & MENOME & $7706-30$ & .00 & 3.1 & .00 & 450 & 57 & 120 & 37 & 14 & .3 \\
\hline $37 \mathrm{~N}$ & $26 \mathrm{~W}$ & 190ADA01 & MENOME & $\begin{array}{l}70-11-24 \\
77-05-29\end{array}$ & $\begin{array}{l}.01 \\
.00\end{array}$ & $\begin{array}{l}3.4 \\
2.6\end{array}$ & $\begin{array}{l}.00 \\
.00\end{array}$ & $\begin{array}{l}300 \\
320\end{array}$ & $\begin{array}{l}42 \\
16\end{array}$ & 76 & $\begin{array}{l}29 \\
31\end{array}$ & $\begin{array}{l}3.8 \\
5.5\end{array}$ & $\because 1$ \\
\hline $23 \mathrm{~N}$ & $05 \mathrm{w}$ & 35 CARAO1 & MSAUH & 77 & .01 & 4.2 & .00 & 170 & 0 & 48 & 13 & 11 & .4 \\
\hline $31 \mathrm{~N}$ & $04 \mathrm{E}$ & О5СВОНО1 & MNTMRY & & & 7.2 & .00 & 16 & 11 & $=2+2$ & 8.5 & 1.3 & $\cdot 0$ \\
\hline $11 \mathrm{~N}$ & $15 w$ & $3440 D D 01$ & MUSKGN & $76-06-28$ & .37 & -- & -- & 450 & 73 & 120 & 36 & 180 & 3.7 \\
\hline $02 \mathrm{~N}$ & OBE & $18 D B A D O 1$ & OAKLND & $75-0 b-22$ & .00 & 14 & .00 & 300 & 0 & 79 & 26 & 23 & .6 \\
\hline $03 \mathrm{~N}$ & ORE & ОЗОВАВО1 & OAKLND & &.$\overline{0}$ & 1.8 &.$\overline{00}$ & 230 & $\overline{0}$ & $55^{--}$ & $23^{--}$ & $17^{--}$ & .5 \\
\hline $23 \mathrm{~N}$ & $01 \mathrm{E}$ & 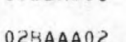 & RGEMAW & 76 & $=$ & -- & $\because$ & -- & $z$ & -- & $-\theta_{2}$ & & - \\
\hline & 0. & OCTAAAUC & OGEMAW & $10-00-24$ & & & & & & & & & \\
\hline & $38 *$ & $3 n \Delta$ & ONTN & $\begin{array}{l}77-09-29 \\
76-05-14\end{array}$ & .01 & 8.1 & .00 & $\begin{array}{l}170 \\
87\end{array}$ & $\begin{array}{l}5 \\
3\end{array}$ & 52 & 10 & $\begin{array}{l}9.0 \\
2.0\end{array}$ & $3^{3}$ \\
\hline $48 \mathrm{Ni}$ & $43 \mathrm{~W}$ & $1 \mathrm{dC}$ & ONIT & 7 & .0 & 4.1 & - & 2 & 45 & 70 & 18 & 4.7 & $\because 1$ \\
\hline $51 \mathrm{~N}$ & & $08408 C 01$ & ONTNG & $\begin{array}{l}75-09-22 \\
76-06-15\end{array}$ & .14 & 2.8 & $\because-$ & 130 & $\begin{array}{l}0 \\
--\end{array}$ & ${ }^{39}-$ & 8.0 & 62 & 2.4 \\
\hline $31 \mathrm{~N}$ & $03 w$ & $34 \mathrm{BCDO01}$ & OTSE & & . & 4.0 & & 22 & 21 & & 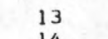 & 10 & .3 \\
\hline $33 \mathrm{~N}$ & 06E & ОВНВ8В01 & PRSQ & & .04 & 1.0 & .0 & 31 & 70 & 10 & 14 & 3.9 & .1 \\
\hline $24 \mathrm{~N}$ & $02 \mathrm{~W}$ & 20तАВА 01 & ROSCMN & $\begin{array}{l}75-06-25 \\
76-06-24\end{array}$ & .06 & 8.1 & .00 & 180 & 1 & 53 & $12^{-}$ & 2.3 & .1 \\
\hline $12 \mathrm{~N}$ & $13 E$ & 33000001 & SANLAC & & .01 & 1.6 & .00 & 290 & 0 & 54 & 38 & 8.7 & .2 \\
\hline $13 N$ & $13 E$ & $12 A D A$ & & & .1 & -- & .00 & $2 \overline{0}$ & 5 & 53 & $19^{-7}$ & 2.1 & \\
\hline $45 \mathrm{~N}$ & $13 w$ & $16 C C C B O 1$ & SCHCFT & $\begin{array}{l}75-09-24 \\
76-06-07\end{array}$ & .00 & - & .00 & 5 & $\begin{array}{l}0 \\
--\end{array}$ & 12 & 5.2 & 110 & 6.7 \\
\hline $47 \mathrm{~N}$ & low & $30+B 8801$ & SCHCFT & 70 & .02 & $\because$ & 0 & 170 & 7 & 41 & 16 & .9 & .0 \\
\hline $04 \mathrm{~S}$ & 16w & & VN $B$ & 7 & & : & & 16 & $\begin{array}{l}4 \\
--\end{array}$ & 40 & & & \\
\hline 025 & $03 E$ & 09i $\triangle A B 02$ & WSHTNW & $\begin{array}{l}75-05-27 \\
76-06-15\end{array}$ & .00 & $\therefore 4$ & .00 & 130 & 30 & ${ }^{37}-$ & 8.7 & ${ }^{14}-$ & 5 \\
\hline $\begin{array}{l}035 \\
21 \mathrm{~N}\end{array}$ & $\begin{array}{l}06 E \\
09 \mathrm{~W}\end{array}$ & $\begin{array}{l}16 \mathrm{BCCDO1} \\
04 \triangle B B C 01\end{array}$ & $\begin{array}{l}\text { WSHINW } \\
\text { WEXFRD }\end{array}$ & $\begin{array}{l}76-06-15 \\
76-06-22\end{array}$ & $\begin{array}{l}.03 \\
.02\end{array}$ & $=$ & $\begin{array}{l}.00 \\
.00\end{array}$ & $\begin{array}{l}710 \\
270\end{array}$ & $\begin{array}{r}350 \\
40\end{array}$ & $\begin{array}{r}200 \\
76\end{array}$ & $\begin{array}{l}52 \\
20\end{array}$ & $\stackrel{14}{9.0}$ & .2 \\
\hline
\end{tabular}


TABLE 3. WATER-QUALITY DATA. (CONTINUED)

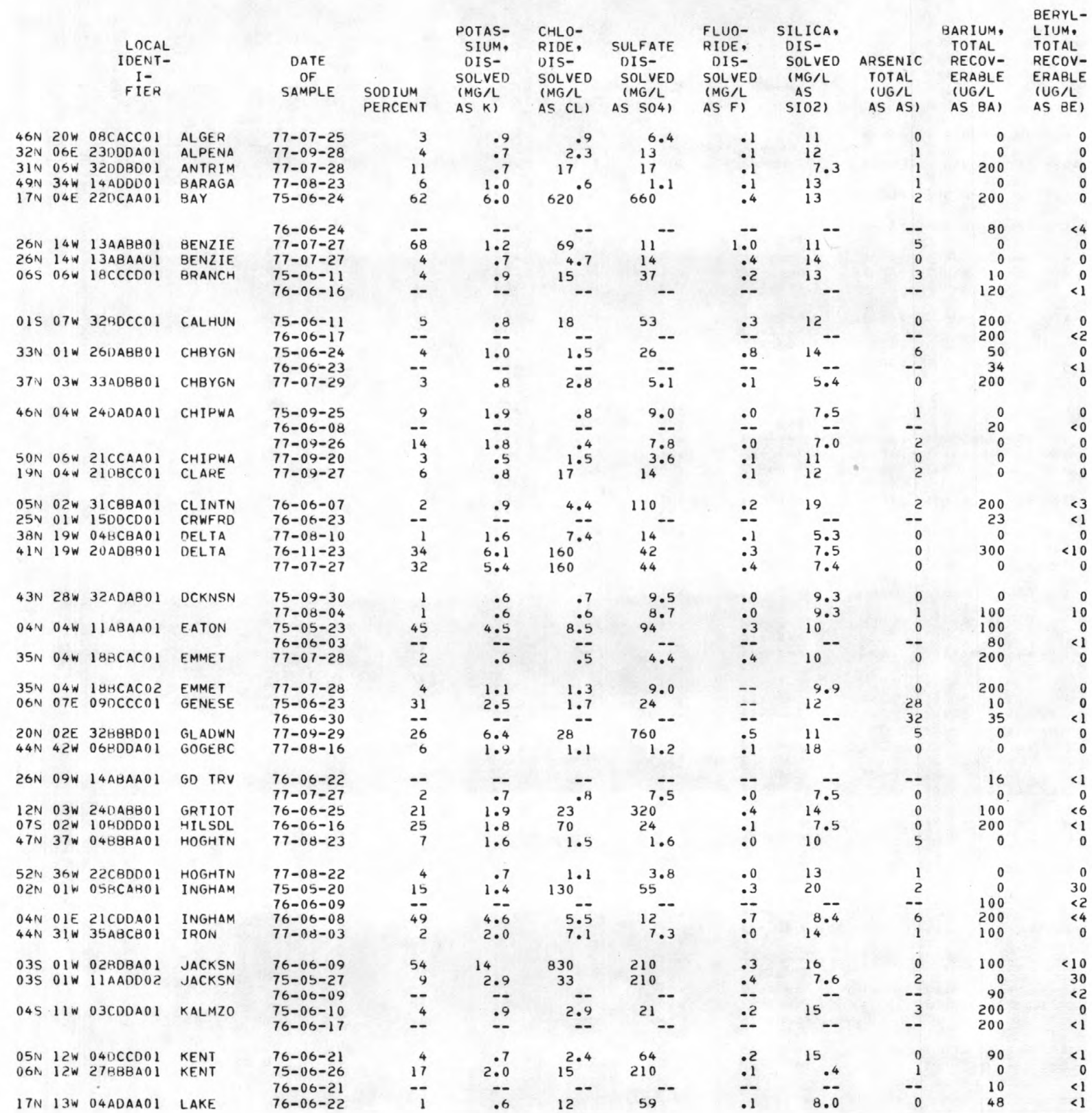


TABLE 3. WATER-QUALITY DATA. (CONTINUED)

\begin{tabular}{|c|c|c|c|c|c|c|c|c|c|c|c|c|c|}
\hline & & $\begin{array}{c}\text { LOCAL } \\
\text { IDENT- } \\
\text { I- } \\
\text { FIER }\end{array}$ & & $\begin{array}{c}\text { DATE } \\
\text { OF } \\
\text { SAMPLE }\end{array}$ & $\begin{array}{l}\text { SODIUM } \\
\text { PERCENT }\end{array}$ & $\begin{array}{l}\text { POTAS- } \\
\text { SIUM, } \\
\text { DIS- } \\
\text { SOLVED } \\
\text { (MG LL } \\
\text { AS K) }\end{array}$ & $\begin{array}{l}\text { CHLO- } \\
\text { RIDE, } \\
\text { DIS- } \\
\text { SOLVED } \\
\text { (MG/L } \\
\text { AS CL) }\end{array}$ & $\begin{array}{l}\text { SULFATE } \\
\text { DIS- } \\
\text { SOLVED } \\
\text { (MG/L } \\
\text { AS S04) }\end{array}$ & $\begin{array}{l}\text { FLUO- } \\
\text { RIDE, } \\
\text { DIS- } \\
\text { SOLVED } \\
\text { (MG/L } \\
\text { AS F) }\end{array}$ & $\begin{array}{l}\text { SILICA, } \\
\text { OIS- } \\
\text { SOLVED } \\
\text { (MG/L } \\
\text { AS } \\
\text { SIOZ) }\end{array}$ & $\begin{array}{l}\text { ARSENIC } \\
\text { TOTAL } \\
\text { (UG/L } \\
\text { AS AS) }\end{array}$ & $\begin{array}{l}\text { BARIUM, } \\
\text { TOTAL } \\
\text { RECOV- } \\
\text { ERABLE } \\
\text { (UG/L } \\
\text { AS BA }\end{array}$ & $\begin{array}{l}\text { BERYL- } \\
\text { LIUM, } \\
\text { TOTAL } \\
\text { RECOV- } \\
\text { ERABLE } \\
\text { (UG/L } \\
\text { AS BE, }\end{array}$ \\
\hline 065 & $04 \mathrm{E}$ & OBDOBA01 & LENAWE & $\begin{array}{l}75-05-27 \\
76-06-14\end{array}$ & 13 & 1.4 & 12 & 160 & .7 & 14. & 15 & $\begin{array}{l}100 \\
200\end{array}$ & $<$ \\
\hline $01 \mathrm{~N}$ & $06 \mathrm{t}$ & 1 ЗОВАВ 01 & LVNGTN & $75-05-22$ & 2 & 1.6 & 27 & 65 & .3 & 11 & 1 & 0 & $\begin{array}{l}10 \\
10 \\
\end{array}$ \\
\hline $49 \mathrm{~N}$ & $12 \mathrm{~W}$ & O1FACBO1 & LUCE & $77-07-26$ & 3 & .9 & 1.1 & $6 . \overline{6}$ & .1 & 11 & 0 & $\begin{array}{r}100 \\
0\end{array}$ & $<1$ \\
\hline $42 \mathrm{v}$ & 02w & O7AABBOI & MACKNC & $\begin{array}{l}76-06-09 \\
77-09-27\end{array}$ & $\begin{array}{l}1 \\
1\end{array}$ & $\begin{array}{l}1.3 \\
1.5\end{array}$ & $\begin{array}{r}1.7 \\
.9\end{array}$ & $\begin{array}{l}9.8 \\
9.5\end{array}$ & .7 & $\begin{array}{l}7.4 \\
6.7\end{array}$ & $\begin{array}{l}0 \\
0\end{array}$ & $\begin{array}{r}10 \\
0\end{array}$ & $<2$ \\
\hline $\begin{array}{l}43 N \\
44 N\end{array}$ & $\begin{array}{l}09 \mathrm{w} \\
26 \mathrm{w}\end{array}$ & $\begin{array}{l}1310 D 0 A 01 \\
23 D A B B 01\end{array}$ & $\begin{array}{l}\text { MACKNC } \\
\text { MARQTE }\end{array}$ & $\begin{array}{l}77-09-22 \\
76-11-22\end{array}$ & $\begin{array}{r}2 \\
2 \\
2\end{array}$ & 1.5 & 1.4 & 39 & .5 & 10 & $\begin{array}{l}0 \\
0\end{array}$ & 10 & $<2$ \\
\hline & & & & $77-09-29$ & 2 & & 1.4 & 16 & .0 & is & 0 & 100 & \\
\hline $47 \mathrm{~N}$ & 26 w & 36880801 & MARQTE & $76-06-01$ & 3 & .7 & 1.1 & 6.6 & .1 & 16 & 0 & 40 & 1 \\
\hline & & & & 76 & 4 & .8 & 1.0 & 6.0 & .1 & 16 & $-\overline{0}$ & 20 & 10 \\
\hline $7 \mathrm{~N}$ & $28 \mathrm{w}$ & $03 c \operatorname{coc} 01$ & MARUTE & $75=09$ & 4 & .0 & 1.0 & 5.5 & .0 & 17 & 22 & & \\
\hline & & & & $76-00$ & -- & -- & $\cdots$ & -- & -- & 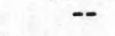 & 12 & 20 & \\
\hline & & & & $\begin{array}{l}76-11-04 \\
77-07-20\end{array}$ & $\begin{array}{l}4 \\
5\end{array}$ & $: 7$ & $\begin{array}{l}2.1 \\
1.1\end{array}$ & $\begin{array}{l}5.0 \\
4.9\end{array}$ & $: 1$ & $\begin{array}{l}14 \\
18\end{array}$ & $\overline{12}$ & $\begin{array}{r}20 \\
0\end{array}$ & $\begin{array}{l}<1 \\
10\end{array}$ \\
\hline & $29 \mathrm{w}$ & OZADDA01 & MARQT & $77-07$ & 5 & 1.2 & .8 & 11 & .1 & 19 & 7 & 0 & \\
\hline 1810 & $17 w$ & $14 C A B A O 1$ & MASON & $77-06$ & 11 & .9 &. .8 & .7 & .3 & ic 5 & 1 & & \\
\hline & & & & & & & & & & & & & \\
\hline $37 \mathrm{~N}$ & 20w & 19DADAOI & MENOME & $76-1$ & 3 & 1.0 & 9.8 & 35 & -1 & 17 & 0 & 70 & $<20$ \\
\hline & $05 \mathrm{w}$ & 35СABA01 & MSAUKE & $\begin{array}{l}77-0 \\
77-0\end{array}$ & 12 & 1. & 10 & 33 & 0 & 17 & 2 & & \\
\hline & $04 \mathrm{E}$ & & MNIME & $77-09$ & $\begin{array}{r}12 \\
2\end{array}$ & .07 & 1.1 & $\begin{array}{l}6.8 \\
7.8\end{array}$ & .2 & 3.6 & 0 & ) & \\
\hline $11 \mathrm{~N}$ & $15 \mathrm{~W}$ & $34 \triangle D D O 01$ & MUSKGN & $76-06-28$ & 46 & 2.8 & $370^{\circ}$ & 2.7 & .2 & 24.0 & -- & 70 & $<10$ \\
\hline $02 \mathrm{~N}$ & OBE & 180BADO1 & OAKLND & $75-05$ & 14 & 1.5 & 32 & 1.4 & .6 & 19 & 0 & & Si \\
\hline $03 \mathrm{~N}$ & $O B E$ & ОЗОВАВО1 & OAKLND & 75 & 14 & 1.2 & 9.8 & $2 . \overline{9}$ & .9 & $18^{-}$ & 6 & 0 & \\
\hline & & & & & & & & & & & -- & & \\
\hline $23 \mathrm{~N}$ & $01 \mathrm{E}$ & OZBAAAOZ & OGEMAW & $76-06-24$ & -- & -- & -- & -- & -- & -- & -- & 17 & $<1$ \\
\hline & $38 w$ & $30 \mathrm{~A}$ & 0 & $\begin{array}{l}77-09-29 \\
76-0 b-14\end{array}$ & $\begin{array}{r}10 \\
5\end{array}$ & 1.1 & ${ }^{16} .7$ & $\begin{array}{l}5.1 \\
5.7\end{array}$ & 00 & $18^{3.9}$ & $\begin{array}{l}1 \\
0\end{array}$ & $\begin{array}{r}0 \\
70\end{array}$ & $<1$ \\
\hline $48 \mathrm{~N}$ & $43 \mathrm{w}$ & & & & 4 & .9 & $41^{\circ}$ & 5.8 & .3 & 21 & 1 & 0 & \\
\hline & & $0830 B C 01$ & ONTNG & $\begin{array}{l}75-09-22 \\
76-06-15\end{array}$ & $\begin{array}{l}49 \\
--\end{array}$ & 7.0 & ${ }^{69}$. & 13 & .3 & 9.2 & -6 & 200 & $<3$ \\
\hline $31 \mathrm{~N}$ & $03 \mathrm{w}$ & $34 \mathrm{BCDDO} 1$ & OTSEG & 7 & 9 & 3. & 20 & 18 & .1 & 7. & 1 & 200 & 0 \\
\hline 33 & OSE & О४АВВВОі & PRSQ I & & 3 & 4.0 & 25 & 28 & .2 & & 0 & & \\
\hline $24 \mathrm{k}$ & $02 \mathrm{w}$ & $20 B A B A 01$ & ROSCMN & $\begin{array}{l}16-0 \\
75-0\end{array}$ & 3 &.$\overline{4}$ & $1 . \overline{4}$ & $4 . \overline{-}$ & $\overline{.} . \overline{2}$ & $7 . \overline{4}$ & 1 & $\begin{array}{r}18 \\
0\end{array}$ & \\
\hline & & & & $76-06$ & -- & $\because$ & & & 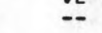 & & -- & 33 & $<1$ \\
\hline $12 \mathrm{~N}$ & $13 E$ & 33000001 & SANLAC & 7 & 6 & 2.3 & 1.5 & 9.9 & .6 & 17 & 18 & 300 & 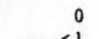 \\
\hline $13 \mathrm{~N}$ & $13 E$ & 12ADAAO1 & SANL & & 2 & .8 & 2.7 & $28^{--}$ & .1 & $10^{-}$ & 4 & & \\
\hline $45 \mathrm{~N}$ & $13 \mathrm{w}$ & $10 \mathrm{CCC} 801$ & SCHCF & $\begin{array}{l}75-09-24 \\
76-06-07\end{array}$ & $8 \overline{2}$ & $\therefore$ & 140 & 16 & - & .3 & -1 & $\begin{array}{l}0 \\
--\end{array}$ & -- \\
\hline N & $16 \mathrm{w}$ & $30 \subseteq B B B 01$ & SCHCFT & & 1 & .4 & .8 & 8.3 & .1 & 8. & 2 & 8 & $<2$ \\
\hline & & & & & - & .4 & & $3=2+3$ & $i$ & 7. & & - & \\
\hline 025 & $03 \mathrm{E}$ & OYDAABOZ & WSHINW & $\begin{array}{l}76-06- \\
75-05-\end{array}$ & 19 & .6 & $\begin{array}{l}4.1 \\
3.2 \\
3.2\end{array}$ & 28 & .1 & il & 4 & $\overline{0}$ & $\begin{array}{l}1 \\
10\end{array}$ \\
\hline & & & & $76-06-15$ & - & $\because$ & -- & - & $\because$ & -- & 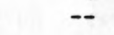 & 50 & $<0$ \\
\hline 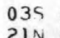 & . & 16ACCDO1 & WSHTNW & $7600-$ & ${ }_{7}^{4}$ & 4. & 5 & $\begin{array}{r}360 \\
37\end{array}$ & $i$ & 10 & 0 & 100 & $<2$ \\
\hline & & & & $76-06-$ & & & & & & & 0 & & \\
\hline
\end{tabular}


TABLE 3. WATER-QUALITY DATA. (CONTINUED)

\begin{tabular}{|c|c|c|c|c|c|c|c|c|c|c|c|c|c|}
\hline & & $\begin{array}{l}\text { LOCAL } \\
\text { IDENT- } \\
\text { I- } \\
\text { FIER }\end{array}$ & & $\begin{array}{c}\text { DATE } \\
\text { OF } \\
\text { SAMPLE }\end{array}$ & $\begin{array}{l}\text { BISMUTH } \\
\text { TOTAL } \\
\text { (UG/L } \\
\text { AS BI) }\end{array}$ & $\begin{array}{l}\text { BORON, } \\
\text { TOTAL } \\
\text { RECOV- } \\
\text { ERABLE } \\
\text { (UG/L } \\
\text { AS B) }\end{array}$ & $\begin{array}{l}\text { CADMIUM } \\
\text { TOTAL } \\
\text { RECOV- } \\
\text { ERABLE } \\
\text { (UG/L } \\
\text { AS CD) }\end{array}$ & $\begin{array}{l}\text { CHRO- } \\
\text { MIUM, } \\
\text { TOTAL } \\
\text { RECOV- } \\
\text { ERABLE } \\
\text { (UG/L } \\
\text { AS CR) }\end{array}$ & $\begin{array}{l}\text { COBALT, } \\
\text { TOTAL } \\
\text { RECOV- } \\
\text { ERABLE } \\
\text { (UG/L } \\
\text { AS CO) }\end{array}$ & $\begin{array}{l}\text { COPPER, } \\
\text { TOTAL } \\
\text { RECOV- } \\
\text { ERABLE } \\
\text { (UG/L } \\
\text { AS CU) }\end{array}$ & $\begin{array}{l}\text { IRON, } \\
\text { TOTAL } \\
\text { RECOV- } \\
\text { ERABLE } \\
\text { (UG/L } \\
\text { AS FE) }\end{array}$ & $\begin{array}{l}\text { IRON, } \\
\text { DIS- } \\
\text { SOLVED } \\
\text { (UG/L } \\
\text { AS FE) }\end{array}$ & $\begin{array}{l}\text { LEAO, } \\
\text { TOTAL } \\
\text { RECOVV- } \\
\text { ERABLE } \\
\text { (UG/L } \\
\text { AS PB) }\end{array}$ \\
\hline $46 \mathrm{~N}$ & $20 \mathrm{w}$ & OBCACCOI & ALGER & $77-07-25$ & -- & 0 & 0 & 10 & 0 & 12 & 40 & 0 & 13 \\
\hline $32 \mathrm{~N}$ & $06 \mathrm{E}$ & $23000 A 01$ & ALPENA & $77-09-28$ & -- & 0 & 0 & $<10$ & 0 & 5 & 850 & 740 & \\
\hline $31 \mathrm{~N}$ & 06w & 32008001 & ANTRIM & $77-07-28$ & -- & 30 & 0 & $<10$ & 0 & 2 & 1100 & - & \\
\hline $49 \mathrm{~N}$ & $34 \mathrm{w}$ & $14 A D D D 01$ & BARAGA & $77-08-23$ & -- & 30 & 2 & 10 & 0 & 12 & 40 & 10 & 16 \\
\hline \multirow[t]{2}{*}{$17 \mathrm{~N}$} & $04 \mathrm{E}$ & $220 C A A D 1$ & BAY & $75-06-24$ & -- & 160 & 0 & 20 & 3 & 7 & 8900 & 1300 & 18 \\
\hline & & & & $76-06-24$ & $<20$ & 100 & -- & $<14$ & $<14$ & $<3$ & 3600 & -- & $<20$ \\
\hline $26 \mathrm{~N}$ & $14 \mathrm{~W}$ & $13 A A B B 01$ & BENZIE & $77-07-27$ & - & 130 & 4 & 10 & 0 & 0 & 100 & 80 & 19 \\
\hline $26 \mathrm{~N}$ & $14 \mathrm{~W}$ & I उABAAOI & BENZIE & $77-07-27$ & -- & 0 & 0 & $<10$ & 3 & 8 & 200 & 160 & \\
\hline \multirow[t]{2}{*}{$06 \mathrm{~S}$} & $06 \mathrm{~W}$ & $18 \mathrm{ccCDO}$ & BRANCH & $75-06-11$ & -- & 20 & 0 & $<10$ & 0 & 1 & 1600 & 850 & 14 \\
\hline & & & & $76-06-16$ & $<4$ & 40 & $<10$ & $<3$ & $<3$ & $<0$ & 1370 & $\cdots$ & \\
\hline \multirow[t]{2}{*}{015} & $07 \mathrm{~W}$ & $32 \mathrm{HOCCOI}$ & CALHUN & $75-06-11$ & -- & 0 & 1 & $<10$ & 0 & 3 & 1600 & 160 & \\
\hline & & & & $76-06-17$ & $<4$ & 20 & $<10$ & $<3$ & $<3$ & 0 & 280 & -- & \\
\hline \multirow[t]{2}{*}{$33 \mathrm{~N}$} & $01 w$ & 26DABBO1 & CHBYGN & $75-06-24$ & -- & 20 & 21 & $<10$ & 0 & 10 & 2700 & 570 & 49 \\
\hline & & & & $76-06-23$ & $<2$ & 20 & $<10$ & $<2$ & $<2$ & 6 & 1200 & - & 10 \\
\hline $37 \mathrm{~N}$ & $03 \mathrm{~W}$ & $33 A D B B 01$ & CHBYGN & $77-07-29$ & -- & 30 & 0 & 10 & 0 & 2 & 200 & 170 & \\
\hline \multirow[t]{3}{*}{$46 \mathrm{~N}$} & $04 \mathrm{w}$ & $240 A 0 A 01$ & CHIPWA & $75-09-25$ & -- & 10 & 2 & 0 & 5 & -- & 23000 & 70 & 190 \\
\hline & & & & $76-06-08$ & $<1$ & 20 & $<10$ & i & $<1$ & -- & 2600 & -- & 20 \\
\hline & & & & $77-09-26$ & - & 30 & 0 & 40 & 0 & -. & 11000 & 150 & 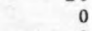 \\
\hline $50 \mathrm{~N}$ & $06 \mathrm{~W}$ & $21 C C A A 01$ & CHIPWA & $77-09-20$ & -- & 50 & 0 & $<10$ & 0 & 0 & - & -- & 0 \\
\hline $19 \mathrm{~N}$ & $04 \mathrm{w}$ & $210 B C C O 1$ & CLARE & $77-09-27$ & -- & 8 & 0 & 10 & 0 & 5 & 340 & 270 & \\
\hline $05 \mathrm{~N}$ & $02 \mathrm{~W}$ & $31 \mathrm{CBBA01}$ & CLINTN & $76-06-07$ & $<10$ & 20 & 0 & $<10$ & $<10$ & 6 & 8900 & 4500 & $<10$ \\
\hline $25 \mathrm{~N}$ & $01 \mathrm{w}$ & $1500 C 001$ & CRWFRD & $76-06-23$ & $<2$ & 20 & $<10$ & $<2$ & $<2$ & 6 & 680 & -- & 30 \\
\hline $38 \mathrm{~V}$ & $19 \mathrm{~W}$ & O4RCBAOL & DELTA & $77-08-10$ & $=$ & 0 & 0 & $<10$ & 0 & 11 & 40 & 80 & 0 \\
\hline \multirow[t]{2}{*}{$41 \mathrm{~N}$} & $19 w$ & 20ADBBOI & DELTA & $76-11-23$ & $<20$ & -- & $<10$ & 180 & 40 & 20 & 460 & 120 & 220 \\
\hline & & & & $77-07-27$ & -- & 110 & 10 & 10 & 0 & 4 & 70 & 90 & 41 \\
\hline \multirow[t]{2}{*}{$43 \mathrm{~N}$} & $28 \mathrm{w}$ & $32 \triangle D A B 01$ & DCKNSN & $75-09-30$ & -- & 0 & 0 & 10 & 0 & 2 & 100 & 20 & 2 \\
\hline & & & & $77-0$ & -- & 0 & 0 & $<10$ & 0 & 5 & 420 & 10 & 12 \\
\hline \multirow[t]{2}{*}{$04 \mathrm{~N}$} & $04 \mathrm{~W}$ & $11 \triangle B A A A 01$ & EATON & $75-05-23$ & -- & 600 & 0 & 0 & 0 & 21 & 3400 & 1500 & 8 \\
\hline & & & & $76-06-03$ & $<4$ & 380 & 17 & $<3$ & $<3$ & 10 & 2600 & -- & 120 \\
\hline $35 \mathrm{~N}$ & $04 \mathrm{~W}$ & $18 B C \triangle C 01$ & EMMET & $77-07-28$ & -- & 0 & 0 & 10 & 0 & 6 & 20 & 10 & \\
\hline \multirow{3}{*}{$\begin{array}{l}35 N \\
06 N\end{array}$} & $04 \mathrm{w}$ & $18 B C A C 02$ & EMMET T & $77-07$ & -- & 0 & 0 & $<1<1$ & 2 & 2 & 4200 & 200 & 12 \\
\hline & $07 \mathrm{E}$ & $090 C C C D 1$ & GENESE & $75-$ & - & 240 & 0 & $<1$ & 0 & 2 & 240 & 140 & \\
\hline & & & & $76-06-30$ & $<4$ & 180 & -- & $<3$ & $<3$ & $<0$ & 270 & - & $<10$ \\
\hline $20 \mathrm{~N}$ & $02 \mathrm{E}$ & $32 B 8 B 001$ & GLAOWN & $77-09-29$ & -- & 370 & 0 & $<10$ & 0 & 11 & 520 & 250 & \\
\hline $44 \mathrm{~N}$ & $42 \mathrm{w}$ & OSBDDA01 & GOGEBC & $77-08-16$ & -- & 30 & 2 & 10 & 0 & 11 & 2000 & 70 & 20 \\
\hline \multirow[t]{2}{*}{$26 \mathrm{~N}$} & $09 \mathrm{w}$ & $14 A B A A 01$ & GO TRV & $06-22$ & $<1$ & 10 & $<10$ & $<$ & $<1$ & $<1$ & 30 & -- & \\
\hline & & & & $77-07-27$ & $\because$ & 0 & 3 & $<10$ & 0 & 0 & 60 & 0 & 13 \\
\hline $12 \mathrm{M}$ & $03 w$ & $240 A B B 01$ & GRTIOT & $76-06-25$ & $<20$ & 90 & -- & $<15$ & $<15$ & 20 & 3700 & 2000 & 20 \\
\hline $07 \mathrm{~s}$ & $02 \mathrm{w}$ & $10 \div D 0001$ & HILSOL & & $<4$ & 20 & 0 & 10 & $<3$ & 120 & 10000 & 360 & 55 \\
\hline $47 \mathrm{~N}$ & $37 \mathrm{w}$ & 04 RBBA01 & HOGHTN & $77-08-23$ & -- & 40 & 0 & 10 & 0 & 7 & 1400 & 20 & 16 \\
\hline $52 \mathrm{~N}$ & $36 \mathrm{w}$ & $22 C B D 001$ & HOGHTN & $77-08$ & -- & 20 & 0 & 1 & 0 & 12 & 130 & 20 & 0 \\
\hline \multirow[t]{2}{*}{$02 \mathrm{~N}$} & $01 \mathrm{w}$ & O5BCABOI & INGHAM & $75-05-$ & -- & 40 & 0 & $<10$ & 0 & 0 & 11000 & -- & 4 \\
\hline & & & & $76-0$ & $<8$ & 20 & 1 & $<6$ & $<6$ & $<2$ & 14000 & 10000 & \\
\hline $04 \mathrm{~N}$ & OIE: & $21 C O D A 01$ & INGHAM & $76-06$ & $<15$ & $\ldots$ & i & 55 & 20 & 16 & 29000 & 520 & $<15$ \\
\hline $44 \mathrm{~N}$ & $31 w$ & $35 \triangle B C B 01$ & IROIN & $77-08-03$ & - & 30 & 0 & 10 & 5 & 5 & 3800 & 10 & 16 \\
\hline & $01 \mathrm{~W}$ & OZBDBA01 & JACKSN & 7 & $<40$ & 190 & 0 & $<4<1$ & $<40$ & 10 & 17 & 2000 & $<40$ \\
\hline \multirow{2}{*}{035} & $01 \mathrm{w}$ & $11 \triangle A A D O 02$ & JACKSN & $75-05-$ & - & 600 & 0 & $<10$ & 1 & 6 & 29 & 2600 & \\
\hline & & & & $76-06-$ & $<7$ & 1000 & 0 & $<5$ & $<5$ & 4 & 4300 & - & 10 \\
\hline \multirow[t]{2}{*}{045} & $11 \mathrm{w}$ & $03 C D D A 01$ & KALMZO & $75-06-10$ & -- & 0 & 0 & 10 & 0 & 2 & 2000 & 590 & 3 \\
\hline & & & & $76-06-17$ & $<3$ & 30 & $<10$ & $<3$ & $<3$ & 0 & 280 & - & 2 \\
\hline \multirow{3}{*}{$\begin{array}{l}05 \mathrm{~N} \\
06 \mathrm{~N}\end{array}$} & $12 w$ & $040 C C$ & KENT & & $<3$ & 40 & $<10$ & $<2$ & $<2$ & 0 & 20 & 1400 & 10 \\
\hline & $12 \mathrm{w}$ & $27 B B B A 01$ & KENT & $75-06-26$ & -- & 160 & 0 & $<10$ & 0 & 7 & 3300 & 660 & \\
\hline & & & & $76-06-21$ & $<4$ & 150 & $<10$ & $<3$ & $<3$ & 4 & 1300 & -- & 10 \\
\hline $17 \mathrm{~N}$ & $13 \mathrm{w}$ & $04 \triangle D A A 01$ & LAKE & $76-06-22$ & $<2$ & 10 & $<10$ & $<2$ & $<2$ & 10 & 5800 & 240 & \\
\hline
\end{tabular}


TABLE 3. WATER-QUALITY DATA. (CONTINUED)

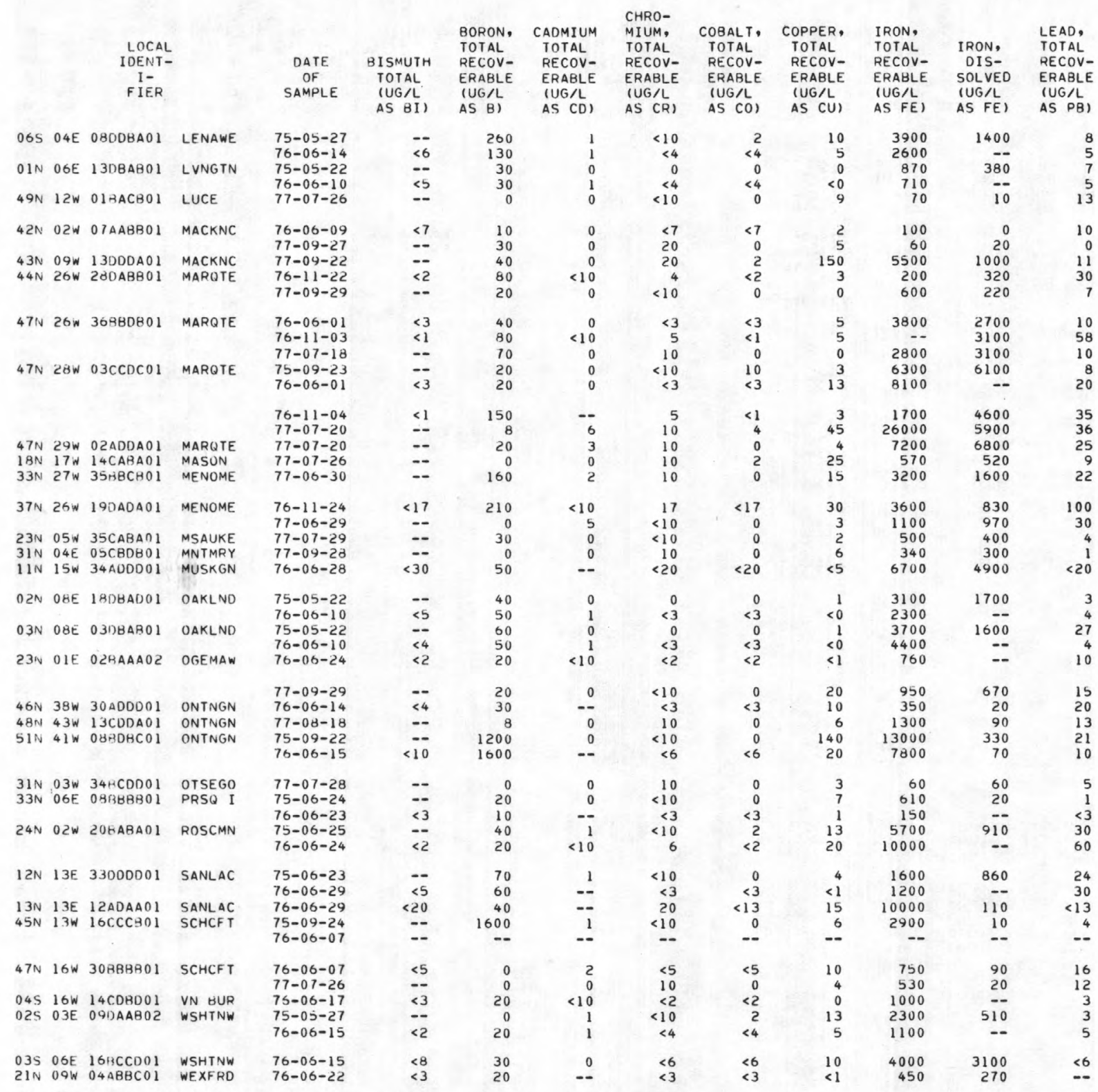


TABLE 3. WATER-QUALITY DATA. (CONTINUED)

\begin{tabular}{|c|c|c|c|c|c|c|c|c|c|c|c|c|c|}
\hline & & $\begin{array}{l}\text { LOCAL } \\
\text { IDENT- } \\
\text { I- } \\
\text { FIER }\end{array}$ & & $\begin{array}{c}\text { DATE } \\
\text { OF } \\
\text { SAMPLE }\end{array}$ & $\begin{array}{l}\text { MANGA- } \\
\text { MESE, } \\
\text { SUS- } \\
\text { PENDED } \\
\text { RECOV. } \\
\text { (UG/L } \\
\text { AS MN) }\end{array}$ & $\begin{array}{l}\text { MANGA- } \\
\text { NESE, } \\
\text { TOTAL } \\
\text { RECOV- } \\
\text { ERABLE } \\
\text { (UG/L } \\
\text { AS MN) }\end{array}$ & $\begin{array}{l}\text { MANGA- } \\
\text { NESE, } \\
\text { DIS- } \\
\text { SOLVED } \\
\text { (UG/L } \\
\text { AS MN) }\end{array}$ & $\begin{array}{l}\text { MOLYB- } \\
\text { DENUM, } \\
\text { TOTAL } \\
\text { RECOV- } \\
\text { ERABLE } \\
\text { (UG,L } \\
\text { AS MO) }\end{array}$ & $\begin{array}{l}\text { NICKEL, } \\
\text { TOTAL } \\
\text { RECOV- } \\
\text { ERABLE } \\
\text { (UG/L } \\
\text { AS NI) }\end{array}$ & $\begin{array}{l}\text { SILVER, } \\
\text { TOTAL } \\
\text { RECOV- } \\
\text { ERABLE } \\
\text { (UG/L } \\
\text { AS AG) }\end{array}$ & $\begin{array}{l}\text { STRON- } \\
\text { TIUM, } \\
\text { TOTAL } \\
\text { RECOV- } \\
\text { ERABLE } \\
\text { (UGAL } \\
\text { AS SR) }\end{array}$ & $\begin{array}{l}\text { VANA- } \\
\text { DIUM, } \\
\text { TOTAL } \\
\text { (UGAL } \\
\text { AS V) }\end{array}$ & $\begin{array}{l}\text { ZINC, } \\
\text { TOTAL } \\
\text { RECOV- } \\
\text { ERABLE } \\
\text { (UG/L } \\
\text { AS ZN) }\end{array}$ \\
\hline $46 \mathrm{~N}$ & $20 \mathrm{w}$ & $08 C A C C D 1$ & ALGER & $77-07-25$ & 0 & 0 & 0 & 0 & 4 & 0 & 40 & -- & 90 \\
\hline $32 \mathrm{~N}$ & $06 \mathrm{E}$ & $23000 \Delta 01$ & ALPENA & $77-09-28$ & 0 & 140 & 140 & 5 & 2 & 0 & bo & -- & 10 \\
\hline $31 \mathrm{~N}$ & $06 \mathrm{w}$ & 32008001 & ANTRIM & $77-07-28$ & 0 & 30 & 30 & 2 & 6 & 0 & 150 & -- & 120 \\
\hline $49 \mathrm{~N}$ & $34 \mathrm{w}$ & $14 A D D D 01$ & BARAGA & $77-08-23$ & 0 & 30 & 30 & 1 & 0 & 0 & 80 & -- & 610 \\
\hline $17 \mathrm{~N}$ & $04 E$ & Z2OCAAOI & BAY & $75-06-24$ & -- & 170 & -- & 0 & 10 & 1 & 4200 & 11 & 2600 \\
\hline & & & & $76-06-24$ & -- & 150 & -- & 20 & $<14$ & $<2$ & 5000 & $<10$ & -- \\
\hline $26 \mathrm{~N}$ & $14 w$ & $13 \triangle A B B 01$ & BENZIE & $77-07-27$ & 0 & 10 & 10 & 11 & 8 & 0 & 620 & -- & 40 \\
\hline $26 \mathrm{~N}$ & $14 w$ & $13 A B A A 01$ & BENZIE & $77-07-27$ & 0 & 10 & 10 & 1 & 9 & 0 & 220 & -- & 240 \\
\hline $06 \mathrm{~S}$ & $06 \mathrm{~W}$ & $18 C C C D O 1$ & BRANCH & $75-06-11$ & -- & 60 & -- & 3 & 0 & 0 & 1500 & 11 & 20 \\
\hline & & & & $76-06-16$ & -- & -- & -- & 3 & $<3$ & $<0$ & 170 & $<3.0$ & 10 \\
\hline 015 & $07 w$ & $3280 \mathrm{CC} 01$ & CALHUN & $75-06-11$ & -- & 100 & -- & 0 & 0 & 0 & 110 & 8.3 & 20 \\
\hline 0.0 & 年 & 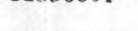 & 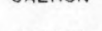 & $76-06-17$ & -- & 110 & -- & 2 & $<3$ & $<0$ & 70 & $<3.0$ & 10 \\
\hline $33 \mathrm{~N}$ & $01 \mathrm{w}$ & 260ABR01 & CHBYGN & $75-06-24$ & -- & 10 & -- & 2 & 2 & $<1$ & 560 & 2.3 & 60 \\
\hline & & & & $76-06-23$ & -- & 10 & -- & 10 & $<2$ & $<0$ & 530 & $<2.0$ & 800 \\
\hline $37 \mathrm{~N}$ & $0.3 w$ & 33ADBBO1 & CHBYGN & $77-07-29$ & 0 & 40 & 40 & 5 & 3 & 0 & 170 & - & 20 \\
\hline $46 \mathrm{~N}$ & $04 \mathrm{w}$ & $240 A D A 01$ & CHIPWA & $75-09-25$ & -- & 20 & -- & 0 & 6 & 0 & 70 & 28 & 520 \\
\hline & & & & $76-06-08$ & -- & 30 & 20 & $<0$ & 2 & $<0$ & 40 & $<.5$ & 340 \\
\hline & & & & $77-09-26$ & 20 & 70 & 50 & 1 & 5 & 0 & 60 & -- & 580 \\
\hline $50 \mathrm{~N}$ & $06 \mathrm{~W}$ & $21 C C A A 01$ & CHIPWA & $77-09-20$ & 0 & 140 & 140 & 0 & 5 & 0 & 50 & -- & 890 \\
\hline $19 \mathrm{~N}$ & $04 w$ & $2108 \mathrm{CC} 01$ & CLARE & $77-09-27$ & 10 & 40 & 30 & 3 & 5 & 0 & 40 & -- & 30 \\
\hline $05 \mathrm{~N}$ & $02 \mathrm{~W}$ & $31 C B B A 01$ & CLININ & $76-06-07$ & -- & 110 & 90 & $<5$ & $<10$ & $<1$ & 150 & $<10$ & 120 \\
\hline $25 \mathrm{~N}$ & $01 \mathrm{w}$ & $150 D C D 01$ & CRWFRD & $76-06-23$ & -- & 10 & -- & 6 & $<2$ & $<0$ & 30 & $<2.0$ & 100 \\
\hline $38 \mathrm{~N}$ & $19 w$ & OABCBAOI & DELTA & $77-08-10$ & 0 & 0 & 0 & 3 & 4 & 0 & 60 & & 310 \\
\hline $41 \mathrm{~N}$ & $19 \mathrm{~W}$ & $20 A D B B 01$ & DELTA & $76-11-23$ & -- & 160 & 40 & $<20$ & $<20$ & 13 & -- & $<40$ & 10 \\
\hline & & & & $77-07-27$ & 0 & 40 & 40 & 1 & 4 & 0 & 1300 & -- & 20 \\
\hline $43 \mathrm{~N}$ & $28 w$ & $32 A D A B 01$ & DCKNSN & $75-09-30$ & -- & 20 & -- & 0 & 0 & 5 & 10 & 1.2 & 100 \\
\hline & & & & $77-08-04$ & 0 & 0 & 0 & 2 & 9 & 0 & 80 & -- & 50 \\
\hline $04 \mathrm{~N}$ & $04 \mathrm{~W}$ & $11 A B A \triangle 01$ & EATON & $75-05-23$ & -- & 20 & -- & 1 & 0 & 0 & 620 & 7.7 & 2800 \\
\hline & & & & $76-06-03$ & -- & 20 & -- & 4 & 6 & $<0$ & 770 & $<3.0$ & 280 \\
\hline $35 \mathrm{~N}$ & $04 \mathrm{~W}$ & $1 B B C A C O 1$ & EMMET & $77-07-28$ & 0 & 10 & 10 & 1 & 5 & 0 & 160 & -- & 10 \\
\hline $35 \mathrm{~N}$ & $04 \mathrm{w}$ & $1 B B C A C O Z$ & EMMET & $77-07-28$ & 0 & 20 & 20 & 3 & 9 & 0 & 50 & -- & 20 \\
\hline $06 \mathrm{~N}$ & $07 E$ & $090 \mathrm{ccc} 01$ & GENESE. & $75-06-23$ & -- & 50 & -- & 22 & 0 & 0 & 660 & 4.5 & 10 \\
\hline & & & & $76-06-30$ & -- & 40 & -- & 40 & $<3$ & $<0$ & 1300 & $<2.0$ & -- \\
\hline $20 N$ & $02 E$ & 32888001 & GLAUWN & $77-09-29$ & 0 & 140 & 140 & 8 & 2 & 0 & 5900 & -- & 750 \\
\hline $44 \mathrm{~N}$ & $42 w$ & OSBDDA01 & GOGEBC & $77-08-16$ & 0 & 210 & -- & 3 & 17 & 0 & 50 & -- & 630 \\
\hline $26 \mathrm{~N}$ & $09 \mathrm{w}$ & $14 A B A A 01$ & GD TRV & & -- & 6 & -- & 5 & $<1$ & $<0$ & 30 & $<1.0$ & $<10$ \\
\hline & & & & & 10 & 10 & 0 & 0 & 4 & 0 & 20 & -- & 20 \\
\hline $12 \mathrm{~N}$ & $03 \mathrm{~N}$ & 241) $\triangle B B$ B 1 & GRTIOT & $76-06-25$ & -- & 100 & 80 & 13 & $<15$ & $<2$ & 1300 & $<15$ & -- \\
\hline 075 & $02 w$ & 10300001 & HILSDL & $76-06$ & -- & 200 & 80 & 4 & 26 & $<0$ & 180 & 4.0 & 440 \\
\hline $47 \mathrm{~N}$ & $37 w$ & $04 B B B \triangle 01$ & HOGHTN & $77-08-23$ & 0 & 20 & 20 & 2 & 7 & 0 & 110 & -- & 130 \\
\hline $52 \mathrm{~N}$ & $36 \mathrm{w}$ & $22 \mathrm{CBD0001}$ & HOGHTN & 77 & 0 & 10 & 10 & 2 & 3 & 0 & so & -- & 290 \\
\hline $02 \mathrm{~N}$ & $01 \mathrm{w}$ & OSВCABOL & INGHAM & $75-0 b-20$ & -- & 250 & $\because$ & 0 & 4 & 0 & 130 & 18 & 20 \\
\hline & & & & $76-06-09$ & -- & 330 & 240 & 4 & $<6$ & $<0$ & 150 & $<6.0$ & 20 \\
\hline $04 \mathrm{~N}$ & $01 E$ & $21 \mathrm{CODAO} 1$ & INGHAM & $76-06-08$ & -- & 740 & 20 & 10 & 50 & $<2$ & 1100 & 50 & 300 \\
\hline $44 \mathrm{~N}$ & $31 \mathrm{w}$ & $35 \triangle B C B 01$ & IRON & $77-08-03$ & 0 & 140 & 140 & 0 & 3 & 0 & 90 & -- & 980 \\
\hline $03 \mathrm{~S}$ & $01 \mathrm{w}$ & $0 \angle \mathrm{HDBAO} 1$ & JACKSN & $76-06$ & -- & 15 & 160 & $<15$ & $<40$ & $<4$ & 2700 & $<30$ & 0 \\
\hline $03 \mathrm{~S}$ & $01 \mathrm{~W}$ & $11 A A D D 02$ & JACKSN & 75 & - & 640 & -- & 0 & 0 & 0 & 230 & 13 & 1000 \\
\hline & & & & $76-00-09$ & -- & 1100 & 710 & 7 & 10 & $<0$ & 290 & 8.0 & 1800 \\
\hline 045 & $11 w$ & $03 C 0 D A 01$ & KALMZO & $75-06-10$ & -- & 160 & -- & 0 & 0 & 0 & 100 & -- & 80 \\
\hline & & & & $76-00-17$ & -- & 110 & -- & 2 & $<2$ & $<0$ & 80 & $<2.0$ & 20 \\
\hline $05 \mathrm{~N}$ & $12 \mathrm{~W}$ & $040 \mathrm{CCD}) 1$ & KENT & $76-$ & -- & 10 & 80 & 2 & $<2$ & $<0$ & 2300 & $<2.0$ & $<30$ \\
\hline $06 \mathrm{~N}$ & $12 W$ & 27RВBА01 & KENT & $75-06-26$ & -- & 190 & -- & 0 & 1 & 1 & 600 & 2.7 & 0 \\
\hline & & & & $76-06-21$ & -- & 770 & -- & $<1$ & $<3$ & $<0$ & 1700 & $<3.0$ & 20 \\
\hline $17 \mathrm{~N}$ & $13 w$ & 04ADAAO1 & LAKE & $76-06-22$ & -- & 180 & 70 & 6 & $<2$ & $<0$ & 40 & $<2.0$ & $<10$ \\
\hline
\end{tabular}


TABLE 3. WATER-QUALITY DATA. (CONTINUed)

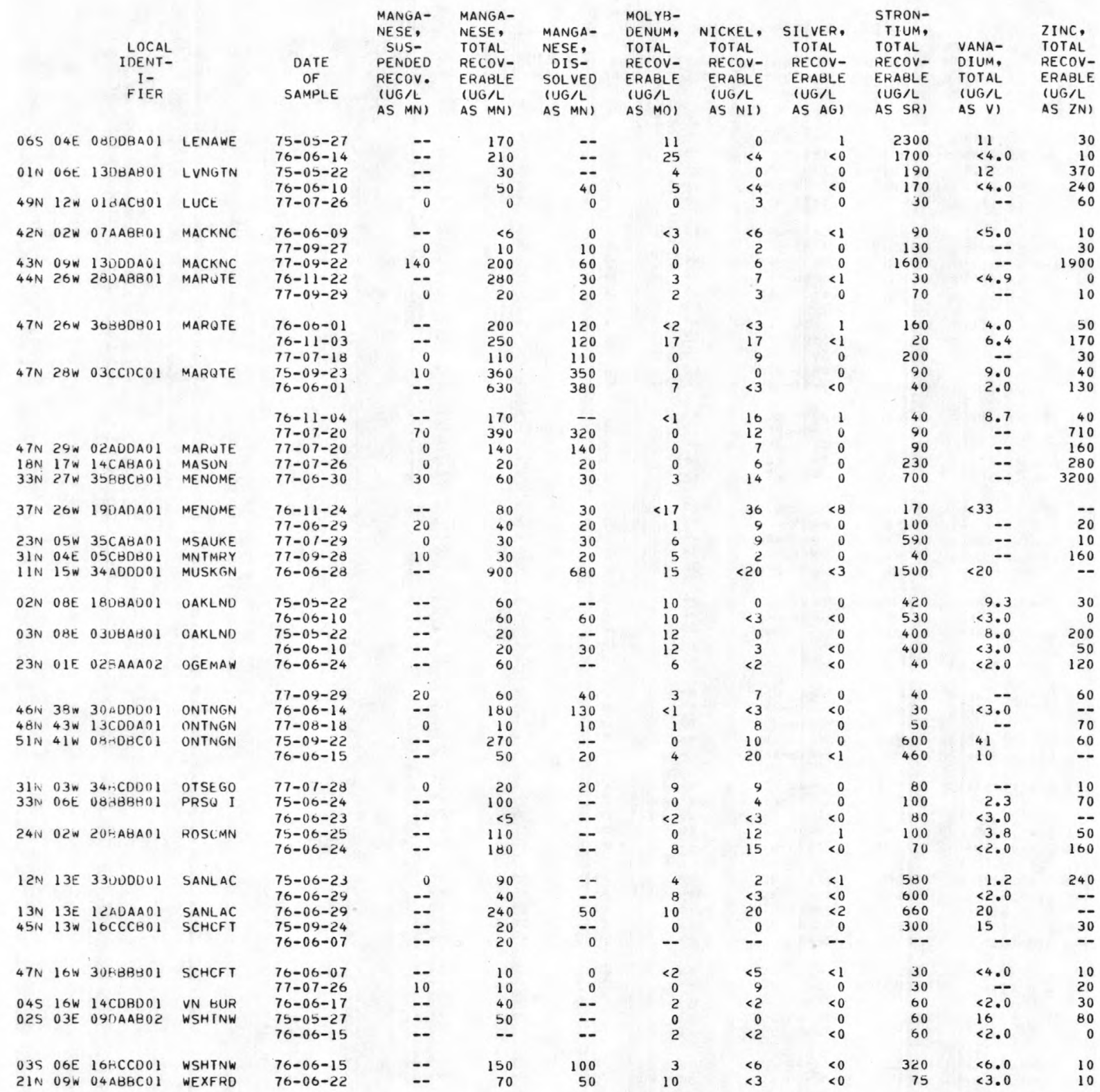


TABLE 3. WATER-QUALITY DATA. (CONTINUED)

\begin{tabular}{|c|c|c|c|c|c|c|c|c|c|c|c|c|c|}
\hline & & $\begin{array}{l}\text { LOCAL } \\
\text { IDENT- } \\
\text { I- } \\
\text { FIER }\end{array}$ & & $\begin{array}{c}\text { DATE } \\
\text { OF } \\
\text { SAMPLE }\end{array}$ & $\begin{array}{l}\text { TIN, } \\
\text { TOTAL } \\
\text { RECOV- } \\
\text { ERABLE } \\
\text { (UG/L } \\
\text { AS SN) }\end{array}$ & $\begin{array}{l}\text { ALUM- } \\
\text { INUM, } \\
\text { TOTAL } \\
\text { RECOV- } \\
\text { ERABLE } \\
\text { (UG/L } \\
\text { AS AL) }\end{array}$ & $\begin{array}{l}\text { GALLIUM } \\
\text { TOTAL } \\
\text { (UG/L } \\
\text { AS GA) }\end{array}$ & $\begin{array}{l}\text { GER- } \\
\text { MANIUM, } \\
\text { TOTAL } \\
\text { (UG/L } \\
\text { AS GE) }\end{array}$ & $\begin{array}{l}\text { LITHIUM } \\
\text { TOTAL } \\
\text { RECOV- } \\
\text { ERABLE } \\
\text { (UG/L } \\
\text { AS LI) }\end{array}$ & $\begin{array}{l}\text { SELE- } \\
\text { NIUM, } \\
\text { TOTAL } \\
\text { (UG/L } \\
\text { AS SE) }\end{array}$ & $\begin{array}{l}\text { TI- } \\
\text { TANIUM, } \\
\text { TOTAL } \\
\text { (UG/L } \\
\text { AS TI) }\end{array}$ & $\begin{array}{l}\text { ZIR- } \\
\text { CONIUM, } \\
\text { TOTAL } \\
\text { (UG/L } \\
\text { AS ZR) }\end{array}$ & $\begin{array}{l}\text { PHENOLS } \\
\text { (UG/L) }\end{array}$ \\
\hline $46 \mathrm{~N}$ & $20 \mathrm{~W}$ & $0 B C A C C 01$ & ALGER & $77-07-25$ & -- & 20 & -- & -- & 0 & 0 & -- & -- & 0 \\
\hline $32 \mathrm{~N}$ & $06 E$ & $23000 A 01$ & ALPENA & $77-0 y-28$ & -- & 130 & -- & -- & 0 & 0 & -- & -- & 2 \\
\hline $31 \mathrm{~N}$ & $06 \mathrm{w}$ & 32008001 & ANTRIM & $77-07-28$ & -- & 50 & -- & -- & 0 & 0 & -- & -- & 0 \\
\hline $49 \mathrm{~N}$ & $34 \mathrm{~W}$ & 14400001 & BARAGA & $77-08-23$ & -- & 30 & -- & -- & 0 & 0 & -- & -- & 0 \\
\hline \multirow[t]{2}{*}{$17 \mathrm{~N}$} & $04 E$ & $221) C A A \cup 1$ & BAY & $75-06-24$ & -- & 440 & -- & -- & 80 & 0 & -- & -- & 1 \\
\hline & & & & $76-06-24$ & $<15$ & 2100 & $<6$ & $<30$ & -- & -- & 140 & $<30$ & -- \\
\hline $26 \mathrm{~N}$ & $14 \mathrm{w}$ & $13 \triangle A B B 01$ & BENZIE & $77-07-27$ & -- & 40 & -- & -- & 0 & 0 & -- & -- & 0 \\
\hline $26 N$ & $14 \mathrm{~W}$ & $13 A B A A O 1$ & BENZIE & $77-07-27$ & -- & 30 & -- & -- & 0 & 0 & -- & -- & 0 \\
\hline \multirow[t]{2}{*}{065} & $06 \mathrm{~W}$ & $18 c C C 001$ & BRANCH & $75-06-11$ & -- & 20 & -- & -- & 0 & 0 & -- & -- & 0 \\
\hline & & & & $76-06-16$ & $<3$ & 10 & $<1$ & -- & $<10$ & -- & 2 & $<6$ & -- \\
\hline \multirow{2}{*}{015} & $07 \mathrm{~W}$ & $32 \mathrm{BOCC} 01$ & CALHUN & $75-06-11$ & -- & 0 & -- & -- & 0 & 0 & -- & -- & 2 \\
\hline & . & 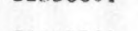 & С & $76-06-17$ & $<3$ & 10 & $<2$ & $<4$ & $<10$ & -- & $<2$ & $<6$ & $=$ \\
\hline \multirow[t]{2}{*}{$33 \mathrm{~N}$} & $01 \mathrm{w}$ & $260 A B B 01$ & CHBYGN & $75-06-24$ & -- & 30 & -- & -- & 1 & 0 & -- & -- & 1 \\
\hline & & & & $76-06-23$ & $<2$ & 20 & $<1$ & $<4$ & $<10$ & -- & $<2$ & $<3$ & - \\
\hline $37 \mathrm{~N}$ & $03 w$ & 33ADBB 01 & CHBYGN & $77-07-29$ & -- & 40 & -- & -- & 0 & 0 & -- & -- & 0 \\
\hline \multirow{3}{*}{$46 \mathrm{~N}$} & $04 \mathrm{w}$ & $240 A D A 01$ & CHIPWA & $75-09-25$ & -- & 0 & -- & -- & 0 & 0 & -- & -- & 0 \\
\hline & (1) & Cका & Cont जा & $76-06-08$ & $<0$ & 10 & $<1$ & $<1$ & 0 & -- & $<0$ & $<1$ & -- \\
\hline & & & & $77-09-26$ & -- & 0 & -- & -- & 0 & 0 & -- & -- & 2 \\
\hline $50 \mathrm{~N}$ & $06 \mathrm{~W}$ & $21 C C A A O 1$ & CHIPWA & $77-09-20$ & -- & 0 & -- & -- & 0 & 0 & -- & -- & 0 \\
\hline $19 \mathrm{~N}$ & $04 \mathrm{w}$ & $2110 \mathrm{BCC} 01$ & CLARE & $77-09-27$ & -- & 40 & -- & -- & 0 & 0 & -- & -- & 4 \\
\hline $05 \mathrm{~N}$ & $02 \mathrm{w}$ & 31C $\triangle B A O 1$ & CLINTN & $76-06-07$ & $<10$ & 40 & $<5$ & $<20$ & 0 & 0 & $<7$ & $<20$ & 0 \\
\hline $25 N$ & $01 w$ & $1500 C D 01$ & CRWFRD & $76-06-23$ & $<2$ & 20 & $<1$ & $<3$ & $<10$ & -- & $<1$ & $<2$ & -- \\
\hline $38 \mathrm{~N}$ & $19 \mathrm{~W}$ & O4BCBA01 & OELTA & $77-08-10$ & -- & 70 & -- & -- & 0 & 0 & -- & -- & 0 \\
\hline \multirow{2}{*}{$41 \mathrm{~N}$} & $19 \mathrm{~W}$ & $20 \triangle D B B 01$ & DELTA & $76-11-23$ & $<30$ & 1200 & $<20$ & $<50$ & 40 & 0 & 370 & $<40$ & 0 \\
\hline & & & & $77-07-27$ & -- & 30 & -- & -- & 30 & 0 & -- & -- & 0 \\
\hline \multirow[t]{2}{*}{$43 \mathrm{~N}$} & $28 \mathrm{w}$ & $32 A D A B 01$ & DCKNSN & $75-09-30$ & -- & 10 & -- & -- & 0 & 0 & -- & -- & 2 \\
\hline & & & & $77-08-04$ & -- & 90 & -- & -- & 0 & 0 & -- & -- & 0 \\
\hline \multirow[t]{2}{*}{$04 \mathrm{~N}$} & $04 \mathrm{~W}$ & $11 A B A A 01$ & EATON & $75-05-23$ & -- & 80 & -- & -- & 40 & 0 & -- & -- & 4 \\
\hline & $8+m$ & 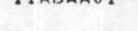 & Сस 10 IV & $76-06-03$ & $<3$ & 60 & $<2$ & $<6$ & 30 & -- & 10 & $<6$ & 0 \\
\hline $35 \mathrm{~N}$ & $04 \mathrm{~W}$ & $1 \mathrm{BHCACO1}$ & EMMET & $77-07-28$ & -- & 60 & $=$ & -- & 0 & 0 & 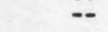 & -- & 0 \\
\hline \multirow{3}{*}{$\begin{array}{l}35 \mathrm{~N} \\
06 \mathrm{~N}\end{array}$} & $04 \mathrm{~W}$ & $1 \triangle B C A C O 2$ & EMMET & $77-07-28$ & -- & 40 & -- & -- & 0 & 0 & -- & -- & 0 \\
\hline & 07E & $090 \mathrm{CcC} 01$ & GENESE & $75-06-23$ & -- & 20 & -- & -- & 20 & 0 & -- & -- & 4 \\
\hline & & & & $76-06-30$ & $<3$ & 16 & $<1$ & $<5$ & - & -- & $<2$ & $<5$ & 0 \\
\hline \multirow{2}{*}{$\begin{array}{l}20 \mathrm{~N} \\
44 \mathrm{~N}\end{array}$} & $02 E$. & $32 \mathrm{~B} 8 \mathrm{B0} 01$ & GLADWN & $77-09-29$ & -- & 30 & $\ldots$ & -- & 10 & 0 & -- & -- & 1 \\
\hline & $42 \mathrm{~W}$ & 06BDOAOI & GOGEBC & $77-08-16$ & -- & 130 & -- & -- & 0 & 0 & -- & -- & 0 \\
\hline \multirow[t]{2}{*}{$26 \mathrm{~N}$} & 09w & $14 A B A A 01$ & GD TRV & $76-06-22$ & $<1$ & 10 & $<1$ & $<2$ & $<10$ & -- & $<1$ & $<2$ & -- \\
\hline & & & & $77-07-27$ & -- & 30 & -- & -- & 0 & 0 & -- & -- & 0 \\
\hline \multirow{3}{*}{$\begin{array}{l}12 \mathrm{~N} \\
07 \mathrm{~S} \\
47 \mathrm{~N}\end{array}$} & $03 w$ & $240 A B B 01$ & GRTIOT & $76-06-25$ & $<15$ & 70 & $<6$ & $<20$ & -- & 0 & $<10$ & $<30$ & 1 \\
\hline & $02 w$ & $10 \times 00001$ & HILSOL & $76-06-16$ & $<3$ & 10000 & $<1$ & $<6$ & 0 & 0 & 140 & $<6$ & 0 \\
\hline & $37 w$ & $04 B \triangle B A 01$ & HOGHTN & $77-08-23$ & -- & 60 & -- & -- & 0 & 0 & -- & -- & 0 \\
\hline \multirow{3}{*}{$\begin{array}{l}52 \mathrm{~N} \\
02 \mathrm{~N}\end{array}$} & $36 \mathrm{w}$ & $22 \mathrm{CBDDO} 1$ & HOGHT & $77-08$ & -- & 3 & -- & -- & 0 & 0 & -- & -- & 0 \\
\hline & $01 \mathrm{w}$ & $05 B C A B O 1$ & INGHAM & $75-05-20$ & -- & $4 \mathrm{C}$ & -- & -- & 3 & 0 & -- & -- & 4 \\
\hline & & & & $76-06-09$ & $<6$ & 5 & $<3$ & $<10$ & 0 & -- & $<6$ & $<10$ & 0 \\
\hline \multirow{2}{*}{$\begin{array}{l}04 \mathrm{~N} \\
44 \mathrm{~N}\end{array}$} & $01 E$ & $21 C 00401$ & INGHAM & $76-06$ & $<15$ & - & 10 & $<20$ & 20 & 0 & -- & 80 & 0 \\
\hline & $31 \mathrm{w}$ & ЗكАВСВО1 & IRON & $77-08-03$ & -- & 60 & -- & -- & 0 & 0 & -- & -- & 0 \\
\hline \multirow{3}{*}{$\begin{array}{l}035 \\
035\end{array}$} & $01 \mathrm{w}$ & O2HDBAO1 & & $76-06-09$ & $<40$ & 20 & $<20$ & $<50$ & 10 & 0 & $<30$ & $<50$ & 0 \\
\hline & $01 \mathrm{w}$ & $11 \triangle A D D O 2$ & JACKSN & $75-05-27$ & - & 670 & -- & -- & 0 & 0 & -- & -- & 2 \\
\hline & & & & $76-06-09$ & $<0$ & 2500 & $<2$ & $<10$ & 10 & -- & 150 & $<10$ & -- \\
\hline $04 \mathrm{~S}$ & $11 \mathrm{w}$ & $03 C D D A 01$ & KALMZO & $75-06-10$ & $\because$ & 20 & -- & -- & 0 & 0 & $=$ & -- & 0 \\
\hline & & & & $76-06-17$ & $<2$ & 10 & $<1$ & $<3$ & $<10$ & -- & $<2$ & $<5$ & -- \\
\hline $05 \mathrm{~N}$ & $12 \mathrm{w}$ & $040 C C D O 1$ & KENT & $76-06-c$ & $<2$ & $3 c$ & $<1$ & $<4$ & $<10$ & 0 & 8 & $<4$ & 0 \\
\hline $06 \mathrm{~N}$ & $12 \mathrm{~W}$ & 27BBBA0I & KENT & $75-06-26$ & $\ldots$ & 2( & $\cdots$ & - & 10 & 0 & -- & - & 1 \\
\hline & & & & $76-06-$ & $<$ & 20 & $<1$ & $<6$ & $<10$ & - & $<3$ & $<6$ & - \\
\hline $17 \mathrm{~N}$ & $13 \mathrm{w}$ & 04ADAAO 1 & LAKE & $76-06-22$ & $<2$ & 10 & $<i$ & $<3$ & $<10$ & 0 & $<1$ & $<3$ & 0 \\
\hline
\end{tabular}


TABLE 3. WATER-QUALITY DATA. (CONTINUED)

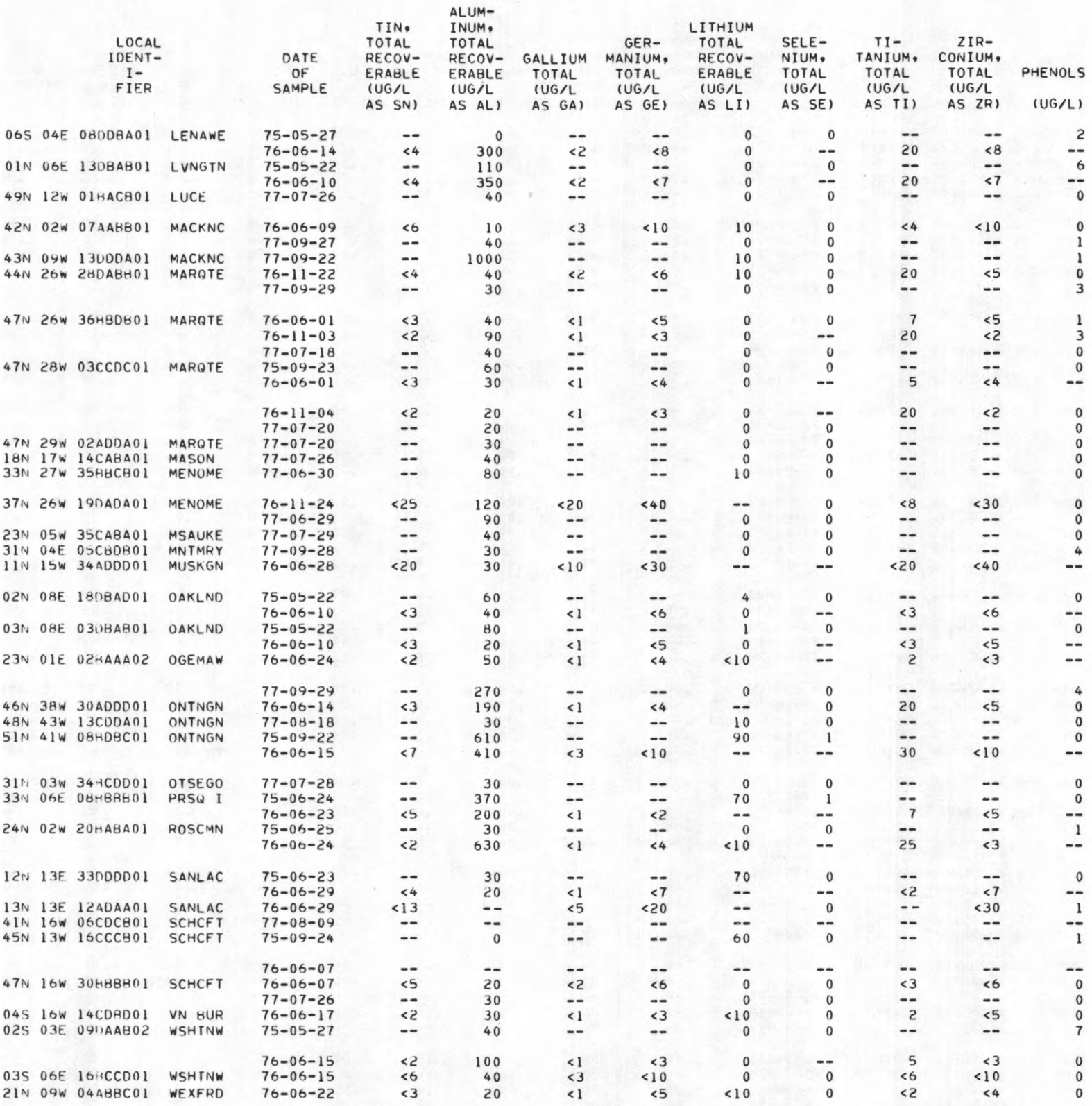


TABLE 3. WATER-QUALITY DATA. (CONTINUED)

\begin{tabular}{|c|c|c|c|c|c|c|c|c|c|c|c|c|c|}
\hline & & $\begin{array}{l}\text { LOCAL } \\
\text { IDENT- } \\
\text { I- } \\
\text { FIER }\end{array}$ & & $\begin{array}{c}\text { DATE } \\
\text { OF } \\
\text { SAMPLE }\end{array}$ & $\begin{array}{l}\text { NAPH- } \\
\text { THA- } \\
\text { LENES, } \\
\text { POLY- } \\
\text { CHLOR. } \\
\text { TOTAL } \\
\text { (UG/L) }\end{array}$ & $\begin{array}{l}\text { ALDRIN, } \\
\text { TOTAL } \\
\text { (UG/L) }\end{array}$ & $\begin{array}{l}\text { LINDANE } \\
\text { TOTAL } \\
\text { (UG/L) }\end{array}$ & $\begin{array}{l}\text { CHLOR- } \\
\text { DANE, } \\
\text { TOTAL } \\
\text { (UG/L) }\end{array}$ & $\begin{array}{l}\text { DOD, } \\
\text { TOTAL } \\
\text { (UG/L) }\end{array}$ & $\begin{array}{l}\text { DDE, } \\
\text { TOTAL } \\
\text { (UG/L) }\end{array}$ & $\begin{array}{l}\text { DDT, } \\
\text { TOTAL } \\
\text { (UG/L) }\end{array}$ & $\begin{array}{l}\text { DI- } \\
\text { ELORIN } \\
\text { TOTAL } \\
\text { (UG/L) }\end{array}$ & $\begin{array}{l}\text { ENOO- } \\
\text { SULFAN, } \\
\text { TOTAL } \\
\text { (UG/L) }\end{array}$ \\
\hline $46 \mathrm{~N}$ & $20 \mathrm{w}$ & $0 B C A C C 01$ & ALGER & $77-07-25$ & .00 & .00 & .00 & .0 & .00 & .00 & .00 & .00 & .00 \\
\hline $32 \mathrm{~N}$ & $06 E$ & $23000 A 01$ & ALPENA & $77-09-28$ & .00 & .00 & .00 & .0 & .00 & .00 & .00 & .00 & .00 \\
\hline $31 \mathrm{~N}$ & Oow & 32008001 & ANTRIM & $\begin{array}{l}77-07-28 \\
777-08-23\end{array}$ & .00 & .00 & .00 & .0 & .00 & .00 & .00 & .00 & .00 \\
\hline $\begin{array}{l}49 \mathrm{~N} \\
17 \mathrm{~N}\end{array}$ & $\begin{array}{l}34 \mathrm{~W} \\
04 \mathrm{E}\end{array}$ & $\begin{array}{l}14 A D D D 01 \\
220 C A A 01\end{array}$ & $\begin{array}{l}\text { BARAGA } \\
\text { BAY }\end{array}$ & $\begin{array}{l}77-08-23 \\
75-06-24\end{array}$ & .00 & $\begin{array}{l}.00 \\
.00\end{array}$ & .00 & $\because 0$ & $\begin{array}{l}.00 \\
.00\end{array}$ & $\begin{array}{l}.00 \\
.00\end{array}$ & $\begin{array}{l}.00 \\
.00\end{array}$ & $\begin{array}{l}.00 \\
.00\end{array}$ & .00 \\
\hline $26 \mathrm{~N}$ & $14 \mathrm{~W}$ & $13 A A B B 01$ & BENZIE & $\begin{array}{l}76-06-24 \\
77-07-27\end{array}$ & $\begin{array}{l}.00 \\
.00\end{array}$ & $\begin{array}{l}.00 \\
.00\end{array}$ & $\begin{array}{l}.00 \\
.00\end{array}$ & .0 & $\begin{array}{l}.00 \\
.00\end{array}$ & $\begin{array}{l}.00 \\
.00\end{array}$ & $\begin{array}{l}.00 \\
.00\end{array}$ & $\begin{array}{l}.00 \\
.00\end{array}$ &.$\overline{0}$ \\
\hline $26 \mathrm{~N}$ & $14 \mathrm{~W}$ & 13 ABAAOI & BENZIE & $77-07-27$ & .00 & .00 & .00 & .0 & .00 & .00 & .00 & .00 & .00 \\
\hline 065 & $06 \mathrm{~W}$ & $18 \mathrm{cccD} 01$ & BRANCH & $75-06-11$ & $=-$ & -- & -- & -- & -- & $=$ & -- & -- & -. \\
\hline & & & & $76-06-16$ & .00 & .00 & .00 & .0 & .00 & .00 & .00 & .00 & -- \\
\hline 015 & $07 \mathrm{~W}$ & $3280 \mathrm{CC} 01$ & CALHUN & $75-06-11$ & -- & .00 & .00 & .0 & .00 & .00 & .00 & .00 & 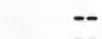 \\
\hline & & & & $76-06-17$ & .00 & .00 & .00 & .0 & .00 & .00 & .00 & .00 & - \\
\hline $33 \mathrm{~N}$ & $01 \mathrm{~W}$ & 260ABB01 & CHBYGN & $75-06-24$ & $=$ & .00 & .00 & .0 & .00 & .00 & .00 & .00 & \\
\hline $37 \mathrm{~N}$ & $03 w$ & $33 \mathrm{ADB} 801$ & CHBYGN & $\begin{array}{l}76-06-23 \\
77-07-29\end{array}$ & .00 & .00 & .00 & -0 & .00 & .00 & .00 & .00 & en \\
\hline & & 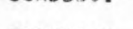 & 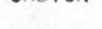 & & .00 & .00 & .00 & $\bullet 0$ & .00 & .00 & .00 & .00 & .00 \\
\hline $46 \mathrm{~N}$ & $04 \mathrm{~W}$ & 240ADA01 & CHIPWA & $\begin{array}{l}75-09-25 \\
76-06-08\end{array}$ & -- & .00 & .00 & .0 & .00 & .00 & .00 & .00 & -- \\
\hline & & & & $\begin{array}{l}76-06-08 \\
77-09-26\end{array}$ & $\overline{--}$ & $=$ & $\overline{--}$ & $=$ & $\overline{--}$ & $\overline{--}$ & $\overline{-}$ & $=$ & - \\
\hline $50 \mathrm{~N}$ & $06 \mathrm{~W}$ & $21 C C A A 01$ & CHIPWA & $77-09-20$ & .00 & .00 & .00 & .0 & .00 & .00 & .00 & .00 & .00 \\
\hline $19 \mathrm{~N}$ & $04 \mathrm{w}$ & $2108 \mathrm{CCO} 01$ & CLARE & $77-09-27$ & .00 & .00 & .00 & .0 & .00 & .00 & .00 & .00 & .00 \\
\hline $05 \mathrm{~N}$ & $02 \mathrm{~W}$ & 31CBBA01 & CLINTN & $76-06-07$ & .00 & .00 & .00 & .0 & .00 & .00 & .00 & .00 & - \\
\hline $25 \mathrm{~N}$ & $01 \mathrm{~W}$ & $1500 C D 01$ & CRWFRD & $76-06-23$ & .00 & .00 & .00 & .0 & .01 & .00 & .00 & .00 & - \\
\hline $38 \mathrm{~N}$ & $19 \mathrm{w}$ & O4BCBAOI & DELTA & $77-08-10$ & .00 & .00 & .00 & .0 & .00 & .00 & .00 & .00 & .00 \\
\hline $41 \mathrm{~N}$ & $19 \mathrm{~W}$ & $20 \mathrm{ADBB} 01$ & DELTA & $76-11-23$ & $\overline{0}$ & $\ddot{0}$ & $\overline{-}$ & $\overline{0}$ & $\overline{0}$ & $\overline{-}$ & $\overline{-}$ & $\ddot{0}$ & $\overline{0}$ \\
\hline & & & & & & & .00 & $\cdot 0$ & $• 00$ & •00 & .00 & .00 & .00 \\
\hline $43 \mathrm{~N}$ & $28 \mathrm{w}$ & 32ADABO1 & DCKNSN & $\begin{array}{l}75-09-30 \\
77-08-04\end{array}$ &.$\overline{00}$ & $\begin{array}{l}.00 \\
.00\end{array}$ & $\begin{array}{l}.00 \\
.00\end{array}$ & $\because 0$ & .00 & .00 & .00 & .00 & $\overline{0}$ \\
\hline $04 \mathrm{~N}$ & $04 \mathrm{w}$ & $11 \triangle B A \Delta O 1$ & EATON & $75-05-23$ & .00 & .00 & $\begin{array}{l}.00 \\
.00\end{array}$ & & .00 & .00 & .00 & .00 & .00 \\
\hline & & & & $76-06-03$ & -- & -- & 0 & 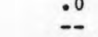 & .00 & .00 & .00 & .00 & $\overline{--}$ \\
\hline $35 \mathrm{~N}$ & $04 \mathrm{~W}$ & $18 B C A C O 1$ & EMMET & $77-07-28$ & .00 & .00 & .00 & .0 & .00 & .00 & .00 & .00 & .00 \\
\hline $35 \mathrm{~N}$ & $04 \mathrm{~W}$ & $18 B C A C O 2$ & EMME T & $77-07-28$ & .00 & .00 & .00 & .0 & .00 & .00 & .00 & .00 & .00 \\
\hline $06 N$ & $07 E$ & $090 \mathrm{ccc} 01$ & GENESE & $\begin{array}{l}75-06-23 \\
76-06-30\end{array}$ &.$\overline{-0}$ & $\begin{array}{l}.00 \\
.00\end{array}$ & $\begin{array}{l}.00 \\
.00\end{array}$ & .0 & $\begin{array}{l}.00 \\
.00\end{array}$ & $\begin{array}{l}.00 \\
.00\end{array}$ & $\begin{array}{l}.00 \\
.00\end{array}$ & $\begin{array}{l}.00 \\
.00\end{array}$ & $=$ \\
\hline $20 \mathrm{~N}$ & $02 E$ & $3288 B 001$ & GLADWN & $77-09-29$ & .00 & .00 & .00 & .0 & .0 & .00 & .00 & .00 & .00 \\
\hline $44 \mathrm{~N}$ & $42 \mathrm{w}$ & $06 \triangle D 0 A 01$ & GOGEBC & $77-08-16$ & .00 & .00 & .00 & .0 & .00 & .00 & .00 & .00 & .00 \\
\hline $26 \mathrm{~N}$ & $09 w$ & $14 A B A A 01$ & GD TRV & $\begin{array}{l}76-06-22 \\
77-07-27\end{array}$ & .00 & .00 & .00 & $\therefore$ & .00 & .00 & .00 & .00 & $=$ \\
\hline $12 \mathrm{~N}$ & $03 w$ & $240 A B B 01$ & GRTIOT & 76 & .00 & .00 & .00 & .0 & .00 & .00 & .00 & .00 & - \\
\hline $07 \mathrm{~s}$ & $02 \mathrm{~W}$ & 10400001 & HILSOL & 76 & .00 & .0 & .0 & • & .0 & .0 & .0 & .00 & - \\
\hline $47 N$ & $37 w$ & $04 B B B A 01$ & HOGHTN & $77-08-23$ & .00 & .00 & .00 & .0 & .00 & .00 & .00 & .00 & .00 \\
\hline $52 \mathrm{~N}$ & $36 \mathrm{w}=$ & $22 C B 0001$ & HOGHTN & $77-0$ & .00 & .00 & .00 & .0 & .0 & .0 & .00 & .00 & .00 \\
\hline $02 \mathrm{~N}$ & $01 \mathrm{WO}$ & $05 B C A B O 1$ & INGHAM & $\begin{array}{l}75-05 \\
76-0 e\end{array}$ & $=$ & .00 & .00 & 0 & .0 & .00 & .00 & .00 & $=$ \\
\hline $04 \mathrm{~N}$ & $01 E$ & $21 C D D A 01$ & INGHAM & $76-06-08$ & .00 & .00 & .00 & .0 & .0 & .0 & .00 &.$\overline{00}$ & - \\
\hline $44 N$ & $31 \mathrm{w}$ & $35 A B C B 01$ & IRON & $77-08-03$ & .00 & .00 & .00 & .0 & .00 & .00 & .00 & .00 & .00 \\
\hline $03 \mathrm{~S}$ & $01 \mathrm{wo}$ & OZBDBA01 & JACKSN & $76-06=$ & .00 & .00 & .00 & .0 & .0 & .0 & .0 & .00 & $=$ \\
\hline 045 & $11 \mathrm{wo}$ & $03 C D D A 01$ & KALMZO & $75-06-10$ & -- & .00 & .00 & .0 & .00 & .00 & .00 &.$\overline{00}$ & 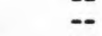 \\
\hline & & & & $76-06-17$ & -- & -- & - & - & -- & -- & -- & -- & 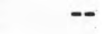 \\
\hline $05 \mathrm{~N}$ & $12 w_{0}$ & $041) C C D 01$ & KENT & $76-$ & .00 & .00 & .0 & .0 & .0 & .0 & .0 & .00 & - \\
\hline $06 \mathrm{~N}$ & $12 W^{2}$ & $27 \mathrm{HBBAO1}$ & KENT & $75-06-26$ & -- & .00 & .00 & .0 & .00 & .00 & $<.01$ & .00 & 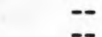 \\
\hline $17 \mathrm{~N}$ & $13 \mathrm{w} 0$ & $04 \triangle D \triangle A 01$ & & $76-06-21$ & .00 & .00 & .00 & .0 & .00 & .00 & .00 & .00 & \\
\hline $17 \mathrm{~N}$ & $13 \mathrm{~N} 0 \mathrm{~d}$ & OUADAAOI & LAKE & & & & .00 & & & & & .00 & \\
\hline
\end{tabular}


TABLE 3. WATER-QUALITY DATA. (CONTINUED)

\begin{tabular}{|c|c|c|c|c|c|c|c|c|c|c|c|c|c|}
\hline & & $\begin{array}{l}\text { LOCAL } \\
\text { IOENT- } \\
\text { I- } \\
\text { FIER }\end{array}$ & & $\begin{array}{c}\text { DAIE } \\
\text { OF } \\
\text { SAMPLE }\end{array}$ & $\begin{array}{l}\text { NAPH- } \\
\text { THA- } \\
\text { LENES, } \\
\text { POLY- } \\
\text { CHLOR. } \\
\text { TOIAL } \\
\text { (UGIL) }\end{array}$ & $\begin{array}{l}\text { ALDRIN, } \\
\text { TOIAL } \\
\text { (UG/L) }\end{array}$ & $\begin{array}{l}\text { LINDANE } \\
\text { TOTAL } \\
\text { (UG/L) }\end{array}$ & $\begin{array}{l}\text { CHLOR- } \\
\text { DANE, } \\
\text { TOTAL } \\
\text { (UG/L) }\end{array}$ & $\begin{array}{l}\text { DUD, } \\
\text { TOTAL } \\
\text { (UGAL) }\end{array}$ & $\begin{array}{l}\text { DOE, } \\
\text { TOTAL } \\
(U G / L)\end{array}$ & $\begin{array}{l}\text { DDT, } \\
\text { TOTAL } \\
\text { (UG/L) }\end{array}$ & $\begin{array}{l}\text { DI- } \\
\text { ELDRIN } \\
\text { TOTAL } \\
\text { (UG/L) }\end{array}$ & $\begin{array}{l}\text { ENDO- } \\
\text { SULFAN, } \\
\text { TOTAL } \\
\text { (UG/L) }\end{array}$ \\
\hline $06 \mathrm{~S}$ & $04 \mathrm{E}$ & O8DOBA01 & LENAWE & & $=$ & .00 & .00 & .0 & .00 & .00 & .00 & .00 & $\overline{-}$ \\
\hline \multirow[t]{2}{*}{$01 \mathrm{~N}$} & $06 E$ & $130 B A B 01$ & LVNGTN & & -- & .00 & .00 & .0 & .00 & .00 & .00 & .00 & -- \\
\hline & & & & $76-06-10$ & -- & - & - & - & - & - & - & - & -- \\
\hline $49 \mathrm{~N}$ & $12 \mathrm{w}$ & $01 B A C B \cap 1$ & LUCE & $77-07-26$ & .00 & .00 & .00 & .0 & .00 & .00 & .00 & .00 & .00 \\
\hline $42 \mathrm{~N}$ & $02 \mathrm{w}$ & $07 \triangle A B Q 01$ & MACKNC & $\begin{array}{l}76-06-09 \\
77-09-27\end{array}$ & $\begin{array}{l}.00 \\
.00\end{array}$ & $\begin{array}{l}.00 \\
.00\end{array}$ & $\begin{array}{l}.00 \\
.00\end{array}$ & .0 & $\begin{array}{l}.00 \\
.00\end{array}$ & $\begin{array}{l}.00 \\
.00\end{array}$ & $\begin{array}{l}.00 \\
.00\end{array}$ & $\begin{array}{l}.00 \\
.00\end{array}$ & .00 \\
\hline $43 \mathrm{~N}$ & $09 \mathrm{w}$ & $13000 A 01$ & MACKNC & $77-09-22$ & .00 & .00 & .00 & .0 & .00 & .00 & .00 & .00 & .00 \\
\hline \multirow[t]{2}{*}{$44 \mathrm{~N}$} & $26 \mathrm{~W}$ & $280 A B B O 1$ & MARQTE & $76-11-22$ & -- & -- & -- & - & -- & -- & -- & -- & -- \\
\hline & & & & $77-09-29$ & .00 & .00 & .00 & .0 & .00 & .00 & .00 & .00 & .00 \\
\hline \multirow[t]{2}{*}{$47 \mathrm{~N}$} & $26 \mathrm{w}$ & 36880801 & MARQTE & $\begin{array}{l}76-06-01 \\
76-11-03\end{array}$ & $\begin{array}{l}.00 \\
.00\end{array}$ & $\begin{array}{l}.00 \\
.00\end{array}$ & $\begin{array}{l}.00 \\
.00\end{array}$ & $\begin{array}{l}.0 \\
.0\end{array}$ & $\begin{array}{l}.00 \\
.00\end{array}$ & $\begin{array}{l}.00 \\
.00\end{array}$ & $\begin{array}{l}.00 \\
.00\end{array}$ & $\begin{array}{l}.00 \\
.00\end{array}$ & $=$ \\
\hline & & & & $77-07-18$ & .00 & .00 & .00 & .0 & .00 & .00 & .00 & .00 & .00 \\
\hline \multirow[t]{4}{*}{$47 \mathrm{~N}$} & $28 w$ & $03 C_{C D C} 01$ & MARQTE & $75-09-23$ & -- & .00 & .00 & .0 & .00 & .00 & .00 & .00 & -- \\
\hline & & & 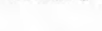 & $76-06-01$ & -- & -- & - & -- & - & -- & -- & -- & 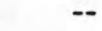 \\
\hline & & & & $76-11-04$ & .00 & .00 & .00 & .0 & .00 & .00 & .00 & .00 & -- \\
\hline & & & & $77-07-20$ & .00 & .00 & .00 & .0 & .00 & .00 & .00 & .00 & .00 \\
\hline $47 \mathrm{~N}$ & $29 \mathrm{w}$ & OटADDDA01 & MARQTE & $77-07-20$ & .00 & .00 & .00 & .0 & .00 & .00 & .00 & .00 & .00 \\
\hline $18 \mathrm{~N}$ & $17 \mathrm{w}$ & $14 C A B \triangle 01$ & MASON & $77-07-26$ & .00 & .00 & .00 & .0 & .00 & .00 & .00 & .00 & .00 \\
\hline $33 \mathrm{~N}$ & $27 \mathrm{w}$ & $35+8 \mathrm{CB} 0.1$ & MENOME & $77-06-30$ & .00 & .00 & .00 & .0 & .00 & .00 & .00 & .00 & .00 \\
\hline $37 \mathrm{~N}$ & $26 \mathrm{~W}$ & 19UADA01 & MENOME & $76-11-24$ & .00 & .00 & .00 & .0 & .00 & .00 & .00 & .00 & -- \\
\hline & & & & $77-06-29$ & .00 & .00 & .00 & .0 & .00 & .00 & .00 & .00 & .00 \\
\hline $23 \mathrm{~N}$ & 05w & $35 \mathrm{CABA01}$ & MSAUKE & $77-07-29$ & .00 & .00 & .00 & .0 & .00 & .00 & .00 & .00 & .00 \\
\hline $31 \mathrm{~N}$ & $04 \mathrm{E}$ & $05 C B 0 B 01$ & MNTMRY & $77-09-28$ & .00 & .00 & .00 & .0 & .00 & .00 & .00 & .00 & .00 \\
\hline $11 \mathrm{~N}$ & $15 \mathrm{w}$ & 34400001 & MUSKGN & $76-06-28$ & -- & -- & -- & -- & -- & -- & -- & -- & -- \\
\hline $02 \mathrm{iv}$ & $08 \mathrm{E}$ & $180 B A D 01$ & OAKLND & $75-05-22$ & -- & .00 & .00 & .0 & .00 & .00 & .00 & .00 & -- \\
\hline & & & & $76-06-10$ & -- & -- & -- & -- & -- & -- & -- & -- & $=-$ \\
\hline $03 \mathrm{~N}$ & $08 \mathrm{E}$ & $0308 A B 01$ & OAKLND & $75-05-22$ & -- & .00 & .00 & .0 & .00 & .00 & .00 & .00 & -- \\
\hline & & & & $76-06-10$ & -- & -- & -- & -- & -- & -- & -- & -- & - \\
\hline $23 \mathrm{~N}$ & $01 E$ & OZZAAA0Z & OGEMAW & $76-06-24$ & .00 & .00 & .00 & .0 & .00 & .00 & .00 & .00 & -- \\
\hline & & & & $77-09-29$ & -- & -- & -- & -- & -- & -- & -- & -- & -- \\
\hline $46 \times \mathrm{N}$ & $38 \mathrm{w}$ & 30400001 & ONTNGN & $76-06-14$ & .00 & .00 & .00 & .0 & .00 & .00 & .00 & .00 & 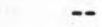 \\
\hline $48 \mathrm{~N}$ & $43 w$ & $13 \mathrm{CDDA} 01$ & ONTNGN & $77-08-18$ & .00 & .00 & .00 & .0 & .00 & .00 & .00 & .00 & .00 \\
\hline $51 \mathrm{~N}$ & $41 \mathrm{w}$ & $08 \mathrm{BOBCOI}$ & ONTNGN & $75-09-22$ & -- & .00 & .00 & .0 & .00 & .00 & .00 & .00 & -- \\
\hline & & & & $76-06-15$ & -- & -- & -- & - & -- & -- & -- & -- & - \\
\hline $31 \mathrm{~N}$ & $03 \mathrm{~W}$ & $34 B C D D 01$ & OTSEGO & $77-07-28$ & .00 & .00 & .00 & .0 & .00 & .00 & .00 & .00 & .00 \\
\hline $33 \mathrm{~N}$ & $06 \mathrm{E}$ & 08888801 & PRSQ I & $75-06-24$ & -- & .00 & .00 & .0 & .00 & .00 & .00 & .00 & - \\
\hline & & & & $76-06-23$ & .00 & .00 & .00 & .0 & .00 & .00 & .00 & .00 & -- \\
\hline $24 \mathrm{~N}$ & $02 \mathrm{~W}$ & 20RABAOI & ROSCMN & $75-06-25$ & $-\bar{x}$ & .00 & .00 & .0 & .00 & .00 & .00 & .00 & -- \\
\hline & & & & $76-06-24$ & .00 & .00 & .00 & .0 & .00 & .00 & .00 & .00 & 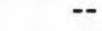 \\
\hline $12 \mathrm{~N}$ & $13 E$ & 33000001 & SANLAC & $75-00-23$ & -- & .00 & .00 & .0 & .00 & .00 & .00 & .00 & -- \\
\hline & & & & & $\overline{0}$ & -- & $-\overline{-}$ & . & $\overline{0}$ & - & $\overline{-}$ & $\overline{0}$ & \\
\hline $13 \mathrm{~N}$ & $13 \mathrm{E}$ & 12ADAAO1 & SANLAC & $76-06-$ & .00 & .00 & .00 & - & .00 & .00 & .00 & .00 & \\
\hline $41 \mathrm{~N}$ & $16 \mathrm{w}$ & $06 C D C 301$ & SCHCF T & $77-08-09$ & .00 & .00 & .00 & $\bullet 0$ & .00 & .00 & .00 & .00 & .00 \\
\hline $45 \mathrm{~N}$ & $13 \mathrm{w}$ & $16 с \mathrm{CCB} 01$ & SCHCFT & $75-09-24$ & -- & .00 & .00 & .0 & .00 & .00 & .00 & .00 & -- \\
\hline & & & & $76-06-07$ & - & -- & -- & - & -- & -- & -- & $=$ & -- \\
\hline $47 \mathrm{iv}$ & low & 30 ВBВB01 & SCHCFT & $76-0$ & .00 & .0 & .0 & - & .00 & .00 & .00 & .00 & \\
\hline & & & & $77-07-$ & - & .0 & .0 & - & .00 & .00 & .00 & .00 & .00 \\
\hline $04 \mathrm{~S}$ & $16 \mathrm{w}$ & $14 \mathrm{COB} D 01$ & VN BUR & $76-06-17$ & .00 & .00 & .00 & .0 & .00 & .00 & .00 & .00 & -- \\
\hline 025 & $03 \mathrm{E}$ & OYOAAB02 & WSHTNW & $75-05-27$ & -- & .00 & .00 & .0 & .00 & .00 & .00 & .00 & - \\
\hline & & & & 76 & -- & -- & -- & -- & -- & -- & - & -- & - \\
\hline $03 \mathrm{~s}$ & $06 \mathrm{E}$ & 16 RCCDO1 & WSHTNW & $76-06-15$ & .00 & .00 & .00 & .0 & .00 & .00 & .00 & .00 & - \\
\hline $21 \mathrm{~N}$ & $09 \mathrm{w}$ & $04 \mathrm{ABBC} 01$ & WEXFRD & $76-06-22$ & .00 & .00 & .00 & .0 & .00 & .00 & .00 & .00 & - \\
\hline
\end{tabular}


TABLE 3. WATER-QUALITY DATA. (CONTINUED)

\begin{tabular}{|c|c|c|c|c|c|c|c|c|c|c|c|c|c|}
\hline & & $\begin{array}{l}\text { LOCAL } \\
\text { IDENT- } \\
\text { I- } \\
\text { FIER }\end{array}$ & & $\begin{array}{c}\text { DATE } \\
\text { OF } \\
\text { SAMPLE }\end{array}$ & $\begin{array}{l}\text { ENDRIN, } \\
\text { TOTALL } \\
\text { (UG/L) }\end{array}$ & $\begin{array}{l}\text { ETHION, } \\
\text { TOIAL } \\
\text { (UG/L) }\end{array}$ & $\begin{array}{l}\text { TOX- } \\
\text { APHENE, } \\
\text { TOTAL } \\
\text { (UG/L) }\end{array}$ & $\begin{array}{l}\text { HEPTA- } \\
\text { CHLOR, } \\
\text { TOTAL } \\
\text { (UG/L) }\end{array}$ & $\begin{array}{l}\text { HEPTA- } \\
\text { CHLOR } \\
\text { EPOXIDE } \\
\text { TOTAL } \\
\text { (UG/L) }\end{array}$ & $\begin{array}{l}\text { METH- } \\
\text { OXY- } \\
\text { CHLOR, } \\
\text { TOTAL } \\
\text { (UG/L) }\end{array}$ & $\begin{array}{l}\text { PCB, } \\
\text { TOTAL } \\
\text { (UG/L) }\end{array}$ & $\begin{array}{l}\text { MALA- } \\
\text { THION, } \\
\text { TOTAL } \\
\text { (UG/L) }\end{array}$ & $\begin{array}{l}\text { PARA- } \\
\text { THION, } \\
\text { TOTAL } \\
\text { (UG/L) }\end{array}$ \\
\hline $46 \mathrm{~N}$ & $20 \mathrm{w}$ & $08 C A C C 01$ & ALGER & $77-07-25$ & .00 & .00 & 0 & .00 & .00 & .00 & .0 & .00 & .00 \\
\hline $32 \mathrm{~N}$ & $06 E$ & 23ODDA01 & ALPENA & $77-09-28$ & .00 & .00 & 0 & .00 & .00 & .00 & .0 & .00 & .00 \\
\hline $31 \mathrm{~N}$ & $06 \mathrm{w}$ & 32008001 & ANTRIM & $77-07-28$ & .00 & .00 & 0 & .00 & .00 & .00 & .0 & .00 & .00 \\
\hline $49 \mathrm{~N}$ & $34 \mathrm{w}$ & $14 A D D D 01$ & BARAGA & $77-08-23$ & .00 & .00 & 0 & .00 & .00 & .00 & .0 & .00 & .00 \\
\hline \multirow[t]{2}{*}{$17 \mathrm{~N}$} & $04 \mathrm{E}$ & $220 C A A 01$ & BAY & $75-06-24$ & .00 & .00 & 0 & .00 & .00 & - & $\cdot 0$ & .00 & .00 \\
\hline & & & & $76-06-24$ & .00 & -- & 0 & .00 & .00 & .00 & .0 & -- & 00 \\
\hline $26 \mathrm{~N}$ & $14 \mathrm{~W}$ & $13 \triangle A B B=1$ & BENZIE & $77-07-27$ & .00 & .00 & 0 & .00 & .00 & .00 & .0 & .00 & .00 \\
\hline $26 \mathrm{~N}$ & $14 \mathrm{w}$ & $13 \triangle B A A O 1$ & BENZIE & $77-07-27$ & .00 & .00 & 0 & .00 & .00 & .00 & .0 & .00 & .00 \\
\hline \multirow[t]{2}{*}{$06 \mathrm{~s}$} & $06 \mathrm{~W}$ & $18 \mathrm{ccc0} 01$ & ARANCH & $75-0 \circ-11$ & $=$ & 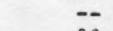 & -- & $\ddot{-}$ & $\ddot{n}$ & $\bar{r}$ & - & 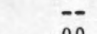 & $-\overline{0}$ \\
\hline & & & & $76-06-16$ & .00 & .00 & 0 & .00 & .00 & .00 & .0 & .00 & .00 \\
\hline 015 & $07 \mathrm{~W}$ & $32 \mathrm{HOCCOl}$ & CALHUN & $75-06-11$ & .00 & .00 & 0 & .00 & .00 & $\overline{0}$ & .0 & .00 & .00 \\
\hline \multirow{2}{*}{$33 \mathrm{~N}$} & $01 \mathrm{w}$ & 261)ABB01 & CHBYGN & $75-06-24$ & .00 &.$\overline{0}$ & 0 & .00 & .00 & - & .0 & .00 & .00 \\
\hline & & & & $76-06-23$ & .00 & - & 0 & .00 & .00 & .00 & .0 & - & \\
\hline $37 \mathrm{~N}$ & 03w & 33ADB801 & CHBYGN & $77-07-29$ & .00 & .00 & 0 & .00 & .00 & .00 & .0 & .00 & .00 \\
\hline \multirow[t]{3}{*}{$46 \mathrm{~N}$} & $04 \mathrm{w}$ & $24 D A D A 01$ & CHIPWA & $75-09-25$ & .00 & .00 & 0 & .00 & .00 & .00 & .0 & .00 & .00 \\
\hline & & & & $76-06-08$ & -- & -- & -- & -- & -- & -- & -- & -- & \\
\hline & & & & $77-09-26$ & -- & -- & -- & -- & -- & -- & -- & -- & - \\
\hline $50 \mathrm{~N}$ & $06 \mathrm{w}$ & $21 C C A A 01$ & CHIPWA & $77-09-20$ & .00 & .00 & 0 & .00 & .00 & .00 & .0 & .00 & .00 \\
\hline $19 \mathrm{~N}$ & $04 \mathrm{~W}$ & $210 B C C O 1$ & CLARE & $77-09-27$ & .00 & .00 & 0 & .00 & .00 & .00 & .0 & .00 & .00 \\
\hline $05 \mathrm{~N}$ & $02 w$ & $31 C B B A 01$ & CLINTN & $76-06-07$ & .00 & .00 & 0 & .00 & .00 & .00 & .0 & .00 & .00 \\
\hline $25 \mathrm{~N}$ & 01 w & 15000001 & CPWF RD & $76-06-23$ & .00 & .00 & 0 & .00 & .00 & .00 & .0 & .00 & .00 \\
\hline $38 \mathrm{~N}$ & $19 \mathrm{w}$ & $04: C B A 01$ & DELTA & $77-08-10$ & .00 & .00 & 0 & .00 & .00 & .00 & .0 & .00 & .00 \\
\hline \multirow[t]{2}{*}{$41 \mathrm{~N}$} & $19 \mathrm{w}$ & $20 \triangle A B B B \cap 1$ & DELTA & $76-11-23$ & -- & - & -- & -- & -- & -- & -- & -- & -- \\
\hline & & & & $77-07-27$ & .00 & .00 & 0 & .00 & .00 & .00 & .0 & .00 & .00 \\
\hline \multirow[t]{2}{*}{$43 \mathrm{~N}$} & $28 w$ & 32ADABO1 & DCKNSN & $75-09-30$ & .00 & .00 & 0 & .00 & .00 & .00 & .0 & .00 & .00 \\
\hline & & & & $77-08-04$ & .00 & .00 & 0 & .00 & .00 & .00 & .0 & .00 & .00 \\
\hline $04 \mathrm{~N}$ & $04 \mathrm{~W}$ & $11 \triangle B A A 01$ & EATUN & $75-05-23$ & .00 & .00 & 0 & .00 & .00 & -- & .0 & .00 & .00 \\
\hline $35 \mathrm{~N}$ & $04 \mathrm{~W}$ & L & EMME T & $76-06-03$ & $\overline{-}$ & -- & $-\overline{0}$ & $\overline{-}$ & $\bar{n}$ & .00 & $\cdots$ & $\bar{x}$ & $\overline{0}$ \\
\hline (1) & $04 \mathrm{w}$ & DOSCACU & EMME I & $77-07-28$ & .00 & .00 & 0 & $\bullet 00$ & .00 & .00 & $\cdot 0$ & .00 & .00 \\
\hline $35 \mathrm{~N}$ & $04 \mathrm{w}$ & $1 \mathrm{BACACO2}$ & EMMET & $77-07-28$ & .00 & .00 & 0 & .00 & .00 & .00 & .0 & .00 & .00 \\
\hline \multirow[t]{2}{*}{$06 \mathrm{~N}$} & $07 E$ & $040 \mathrm{CCC} 01$ & GENESE & $75-06-23$ & .00 & .00 & 0 & .00 & .00 & -- & .0 & .00 & .00 \\
\hline & & & & $76-06-30$ & .00 & -- & 0 & .00 & .00 & .00 & .0 & -- & -- \\
\hline $20 N$ & $02 E$ & згчв8n01 & GLADWN & $77-09-29$ & .00 & .00 & 0 & .00 & .00 & .00 & .0 & .00 & .00 \\
\hline $44 \mathrm{~N}$ & $42 w$ & 06riUDA01 & GOGEBC & $77-08-16$ & .00 & .00 & 0 & .00 & .00 & .00 & .0 & .00 & .00 \\
\hline $26 \mathrm{~N}$ & $09 \mathrm{w}$ & $14 \triangle B A A 01$ & GD TRV & $76-06$ & .00 & .00 & 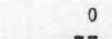 & .00 & .00 & .00 & $\therefore 0$ & .00 & .00 \\
\hline $12 \mathrm{~N}$ & $03 \mathrm{~W}$ & 240 ARRO1 & GRTIOT & $\begin{array}{l}76-0 \\
76-0\end{array}$ & .00 & .00 & 0 & .00 & .00 & .00 & .0 & .00 & \\
\hline $07 \mathrm{~s}$ & $0 \mathrm{ZW}$ & 10 HODOOI & HILSOL & $76-06$ & .00 & .00 & 0 & .00 & .00 & .0 & .0 & .00 & .00 \\
\hline $47 \mathrm{~N}$ & $37 w$ & 04 НBBA01 & HOGHTN & $77-08-23$ & .00 & .00 & 0 & .00 & .00 & .00 & .0 & .00 & .00 \\
\hline \multirow{3}{*}{$02 N$} & $36 w$ & 220.80001 & HOGHTN & $77-0$ & .0 & .0 & 0 & .00 & .00 & .0 & .0 & .00 & .00 \\
\hline & $01 \mathrm{w}$ & OSACABOI & INGHAM & 75 & .00 & .00 & 0 & .00 & .00 & - & .0 & .00 & .00 \\
\hline & & & & & -- & -- & -- & -- & -- & - & 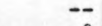 & $=$ & \\
\hline $04 \mathrm{~N}$ & 01E & $21 C D D A 01$ & INGHAM & $76-06-08$ & .00 & .00 & 0 & .00 & .00 & .0 & .0 & .00 & .00 \\
\hline $44 \mathrm{~N}$ & $31 w$ & $35 \triangle \triangle B C B O 1$ & IRON & $77-08-03$ & .00 & .00 & 0 & .00 & .00 & .00 & $\cdot 0$ & .00 & .00 \\
\hline \multirow{3}{*}{035} & $01 \mathrm{w}$ & ОЕНОВАО1 & JACKSN & $76-06$ & .00 & .00 & 0 & .00 & .00 & .0 & .0 & .00 & .00 \\
\hline & $01 \mathrm{w}$ & $11 \triangle A D D 02$ & JACKSN & $75-05-27$ & .00 & .00 & 0 & .00 & .00 & - & .0 & .00 & 00 \\
\hline & & & & $76-0$ & -- & -- & -- & -- & -- & .00 & -- & -- & \\
\hline $04 \mathrm{~S}$ & $11 \mathrm{w}$ & 03 CDDAOL & KALMZO & $\begin{array}{l}75-06-10 \\
76-06-17\end{array}$ & .00 & .00 & -- & .00 & .00 & $\overline{--}$ & $\because 0$ & .00 & .00 \\
\hline & $12 \mathrm{~W}$ & $04 \mathrm{i})(\mathrm{COO}$ & KENT & $76-06-$ & 0 & 0 & 0 & 0 & 0 & .0 & - $1>>3$ & .00 & \\
\hline \multirow[t]{2}{*}{$06 N$} & $12 w$ & 27ABBAOI & KENT & $75-06-26$ & .00 & .00 & 0 & .00 & .00 & - & .0 & .00 & .00 \\
\hline & & & & $76-06-21$ & .00 & -- & 0 & .00 & .00 & .00 & .0 & -- & \\
\hline $\mathbb{N N}$ & $3 w$ & $A D A A 0]$ & LAK & $76-06-22$ & .00 & .00 & & .00 & .00 & .00 & .0 & -- & \\
\hline
\end{tabular}


TABLE 3. WATER-QUALITY DATA. (CONTINUED)

\begin{tabular}{|c|c|c|c|c|c|c|c|c|c|c|c|c|c|}
\hline & & $\begin{array}{l}\text { LOCAL } \\
\text { IDENT- } \\
\text { I- } \\
\text { FIF.R }\end{array}$ & & $\begin{array}{c}\text { DATE } \\
\text { OF } \\
\text { SAMPLE }\end{array}$ & $\begin{array}{l}\text { ENDRIN, } \\
\text { TOTAL } \\
\text { (UG/L) }\end{array}$ & $\begin{array}{l}\text { ETHION, } \\
\text { TOTAL } \\
\text { (UG/L) }\end{array}$ & $\begin{array}{l}\text { TOX- } \\
\text { APHENE, } \\
\text { TOTAL } \\
\text { (UG/L) }\end{array}$ & $\begin{array}{l}\text { HEPTA- } \\
\text { CHLOR, } \\
\text { TOTAL } \\
\text { (UG/L) }\end{array}$ & $\begin{array}{l}\text { HEPTA- } \\
\text { CHLOR } \\
\text { EPOXIDE } \\
\text { TOTAL } \\
\text { (UG } L \text { ) }\end{array}$ & $\begin{array}{l}\text { METH- } \\
\text { OXY- } \\
\text { CHLOR, } \\
\text { TOTAL } \\
\text { (UG L L ) }\end{array}$ & $\begin{array}{l}\text { PCB, } \\
\text { TOTAL } \\
(U G / L)\end{array}$ & $\begin{array}{l}\text { MALA- } \\
\text { THION, } \\
\text { TOTAL } \\
\text { (UGAL) }\end{array}$ & $\begin{array}{l}\text { PARA- } \\
\text { THION, } \\
\text { TOIAL } \\
\text { (UG/L) }\end{array}$ \\
\hline 065 & $04 \mathrm{E}$ & 0800BA01 & LENAWE & $\begin{array}{l}75-05-27 \\
76-06-14\end{array}$ & .00 & .00 & -- & .00 & .00 &.$\overline{00}$ & $\because 0$ & .00 & .00 \\
\hline $01 \mathrm{~N}$ & $06 \mathrm{E}$ & $130 \mathrm{BABO1}$ & LVNGTN & $\begin{array}{l}75-05-22 \\
76-06-10\end{array}$ & .00 & .00 & -- & .00 & .00 &.$\overline{0}$ & $\because 0$ & .00 & .00 \\
\hline $49 \mathrm{~N}$ & $12 w$ & $01 \times A C B 01$ & LUCE & $77-07-26$ & .00 & .00 & 0 & .00 & .00 & .00 & .0 & .00 & .00 \\
\hline $42 \mathrm{~N}$ & $02 \mathrm{~W}$ & $07 A A B B 01$ & MACKNC & $\begin{array}{l}76-06-09 \\
77-09-27 \\
77-09-2\end{array}$ & $\begin{array}{r}.00 \\
.00 \\
.00\end{array}$ & $\begin{array}{l}.00 \\
.00 \\
.00\end{array}$ & $\begin{array}{l}0 \\
0 \\
0\end{array}$ & $\begin{array}{r}.00 \\
.00 \\
.00\end{array}$ & $\begin{array}{r}.00 \\
.00\end{array}$ & $\begin{array}{l}.00 \\
.00 \\
.00\end{array}$ & $\begin{array}{l}.0 \\
.0 \\
0\end{array}$ & $\begin{array}{l}.00 \\
.00 \\
.00\end{array}$ & $\begin{array}{r}.00 \\
.00 \\
.00\end{array}$ \\
\hline $\begin{array}{l}43 \mathrm{~N} \\
44 \mathrm{~N}\end{array}$ & $\begin{array}{l}00 \mathrm{~N} \\
26 \mathrm{w}\end{array}$ & $\begin{array}{l}13000 \Delta 01 \\
280 A B 801\end{array}$ & $\begin{array}{l}\text { MACKNC } \\
\text { MARQTE }\end{array}$ & $\begin{array}{l}77-09-22 \\
76-11-22 \\
77-09-29\end{array}$ & $\begin{array}{l}.00 \\
.00\end{array}$ & .00 & -0 & $\begin{array}{l}.00 \\
.00\end{array}$ & $\begin{array}{l}.00 \\
.00\end{array}$ & $\begin{array}{l}.00 \\
.00\end{array}$ & $\begin{array}{l}.0 \\
-0\end{array}$ & $\begin{array}{l}.00 \\
.00\end{array}$ & .00 \\
\hline $47 \mathrm{~N}$ & $26 \mathrm{~W}$ & 36в80801 & MARQTE & $\begin{array}{l}76-06-01 \\
76-11-03 \\
77-07-18 \\
75-09-23 \\
76-06-01\end{array}$ & $\begin{array}{l}.00 \\
.00 \\
.00 \\
.00 \\
.-\end{array}$ & $\begin{array}{l}.00 \\
.00 \\
.00 \\
.00 \\
.-\end{array}$ & $\begin{array}{r}0 \\
0 \\
0 \\
0 \\
--\end{array}$ & $\begin{array}{l}.00 \\
.00 \\
.00 \\
.00 \\
.-\end{array}$ & $\begin{array}{l}.00 \\
.00 \\
.00 \\
.00 \\
-0\end{array}$ & $\begin{array}{l}.00 \\
.00 \\
.00 \\
.00 \\
-.-\end{array}$ & $\begin{array}{l}.0 \\
.0 \\
.0 \\
.0 \\
--\end{array}$ & $\begin{array}{l}.00 \\
.00 \\
.00 \\
.00 \\
.-\end{array}$ & $\begin{array}{l}.00 \\
.00 \\
.00 \\
.00 \\
.-\end{array}$ \\
\hline $\begin{array}{l}47 N \\
18 N \\
33 N\end{array}$ & $\begin{array}{l}29 w \\
17 w \\
27 w\end{array}$ & $\begin{array}{l}02 A D D A 01 \\
14 C A B A 01 \\
354 B C B 01\end{array}$ & $\begin{array}{l}\text { MARQTE } \\
\text { MASON } \\
\text { MENOME }\end{array}$ & $\begin{array}{l}76-11-04 \\
77-07-20 \\
77-07-20 \\
77-07-26 \\
77-06-30\end{array}$ & $\begin{array}{l}.00 \\
.00 \\
.00 \\
.00 \\
.00\end{array}$ & $\begin{array}{l}.00 \\
.00 \\
.00 \\
.00 \\
.00\end{array}$ & $\begin{array}{l}0 \\
0 \\
0 \\
0 \\
0\end{array}$ & $\begin{array}{l}.00 \\
.00 \\
.00 \\
.00 \\
.00\end{array}$ & $\begin{array}{l}.00 \\
.00 \\
.00 \\
.00 \\
.00\end{array}$ & $\begin{array}{l}.00 \\
.00 \\
.00 \\
.00 \\
.00\end{array}$ & $\begin{array}{l}.1 \\
.0 \\
.0 \\
.0 \\
.0\end{array}$ & $\begin{array}{l}.00 \\
.00 \\
.00 \\
.00 \\
.00\end{array}$ & $\begin{array}{l}.00 \\
.00 \\
.00 \\
.00 \\
.00\end{array}$ \\
\hline $\begin{array}{l}23 \mathrm{~N} \\
31 \mathrm{~N} \\
11 \mathrm{~N}\end{array}$ & $\begin{array}{l}05 w \\
04 E \\
15 w\end{array}$ & $\begin{array}{l}1 \text { YOADA01 } \\
3 \text { SCABA01 } \\
05 \mathrm{CBDBO1} \\
34 \mathrm{ADDO} 01\end{array}$ & $\begin{array}{l}\text { MENOME } \\
\text { MSAUKE } \\
\text { MNTMRY } \\
\text { MUSKGN }\end{array}$ & $\begin{array}{l}76-11-24 \\
77-06-29 \\
77-07-29 \\
77-09-28 \\
76-06-28\end{array}$ & $\begin{array}{l}.00 \\
.00 \\
.00 \\
.00 \\
--\end{array}$ & $\begin{array}{l}.00 \\
.00 \\
.00 \\
.00 \\
--.\end{array}$ & $\begin{array}{r}0 \\
0 \\
0 \\
0 \\
--\end{array}$ & $\begin{array}{l}.00 \\
.00 \\
.00 \\
.00 \\
-.\end{array}$ & $\begin{array}{l}.00 \\
.00 \\
.00 \\
.00 \\
--\end{array}$ & $\begin{array}{l}.00 \\
.00 \\
.00 \\
.00 \\
--.\end{array}$ & $\begin{array}{l}.0 \\
.0 \\
.0 \\
.0 \\
--\end{array}$ & $\begin{array}{l}.00 \\
.00 \\
.00 \\
.00 \\
--\end{array}$ & $\begin{array}{l}.00 \\
.00 \\
.00 \\
.00 \\
--.\end{array}$ \\
\hline $02 \mathrm{~N}$ & $0 R E$ & $181) B A D O 1$ & OAKLND & $\begin{array}{l}75-05-22 \\
76-06-10\end{array}$ & .00 & .00 & -- & .00 & .00 &.$\overline{00}$ & $\because 0$ & .00 & .00 \\
\hline $03 \mathrm{~N}$ & OAE & $030 B A B 01$ & OAKLND & $\begin{array}{l}75-05-22 \\
76-06-10\end{array}$ & .00 & .00 & -0 & .00 & .00 &.$\overline{00}$ & $\therefore 0$ & .00 & .00 \\
\hline $23 \mathrm{~N}$ & $01 E$ & ОГВАAАОZ & OGEMAW & $76-06-24$ & .00 & .00 & 0 & .00 & .00 & .00 & $\cdot 0$ & .00 & .00 \\
\hline $46 \mathrm{~N}$ & $38 \mathrm{~W}$ & $30 A D D D 01$ & ONTNGN & $\begin{array}{l}77-09-29 \\
76-06-14\end{array}$ &.$\overline{0}$ & -- & - & - & - & - & - & -- & -- \\
\hline $48 \mathrm{~N}$ & $43 w$ & $13 C 00 A 01$ & ONTNGN & $77-08-18$ & .00 & .08 & $\begin{array}{l}0 \\
0\end{array}$ & .00 & $\begin{array}{l}.00 \\
.00\end{array}$ & $\begin{array}{l}.00 \\
.00\end{array}$ & .0 & $\begin{array}{l}.00 \\
.00\end{array}$ & $\begin{array}{l}.00 \\
.00\end{array}$ \\
\hline $51 \mathrm{~N}$ & $41 \mathrm{~W}$ & 08 तUBCOI & ONTNGN & $\begin{array}{l}75-09-22 \\
76-06-15\end{array}$ & .00 & .00 & -0 & .00 & .00 & .00 & .0 & .00 & .00 \\
\hline $\begin{array}{l}31 \mathrm{~N} \\
33 \mathrm{~N}\end{array}$ & $\begin{array}{l}03 \mathrm{H} \\
06 E\end{array}$ & $\begin{array}{l}344 C D D 01 \\
03 \text { } А B В \cap 1\end{array}$ & $\begin{array}{l}\text { OTSEGO } \\
\text { PRSQ I }\end{array}$ & $\begin{array}{l}77-07-28 \\
75-06-24\end{array}$ & $\begin{array}{l}.00 \\
.00\end{array}$ & $\begin{array}{l}.00 \\
.00\end{array}$ & $\begin{array}{l}0 \\
0\end{array}$ & .00 & $\begin{array}{l}.00 \\
.00\end{array}$ & .00 & $: 0$ & $\begin{array}{l}.00 \\
.00\end{array}$ & $\begin{array}{l}.00 \\
.00\end{array}$ \\
\hline $24 \mathrm{~N}$ & $02 \mathrm{~W}$ & $20-A B A 01$ & ROSCMN & $\begin{array}{l}76-06-23 \\
75-06-25 \\
76-06-24\end{array}$ & $\begin{array}{l}.00 \\
.00 \\
.00\end{array}$ & .00 & $\begin{array}{l}0 \\
0 \\
0\end{array}$ & $\begin{array}{l}.00 \\
.00 \\
.00\end{array}$ & $\begin{array}{l}.00 \\
.00 \\
.00\end{array}$ & $\begin{array}{l}.00 \\
.00\end{array}$ & $\begin{array}{l}.0 \\
.0 \\
.0\end{array}$ & .00 & .00 \\
\hline $12 \mathrm{iv}$ & $13 E$ & 33000001 & SANLAC & $\begin{array}{l}75-06-23 \\
76-06-29\end{array}$ & .00 & .00 & -0 & .00 & .00 & $=$ & $\because 0$ & .00 & .00 \\
\hline $\begin{array}{l}13 N \\
41 N\end{array}$ & $\begin{array}{l}13 E \\
16 \mathrm{~W}\end{array}$ & $\begin{array}{l}\text { 1 } \angle A D A A 01 \\
06 C O C B O 1\end{array}$ & $\begin{array}{l}\text { SANLAC } \\
\text { SCHCFT }\end{array}$ & $\begin{array}{l}76-06-6 \\
77-08-6\end{array}$ & $\begin{array}{l}.00 \\
.00\end{array}$ & .00 & $\begin{array}{l}0 \\
0\end{array}$ & $\begin{array}{l}.00 \\
.00\end{array}$ & $\begin{array}{l}.00 \\
.00\end{array}$ & $\begin{array}{l}.00 \\
.00\end{array}$ & .0 & $\begin{array}{l}.00 \\
.00\end{array}$ & $\begin{array}{l}.00 \\
.00\end{array}$ \\
\hline $45 \mathrm{~N}$ & $13 \mathrm{w}$ & $16 \mathrm{CCCHOI}$ & SCHCF T & $75-09-24$ & .00 & .00 & 0 & .00 & .00 & .00 & .2 & .00 & .00 \\
\hline $\begin{array}{l}045 \\
025\end{array}$ & $\begin{array}{l}16 \mathrm{~W} \\
03 \mathrm{E}\end{array}$ & $\begin{array}{l}\text { 30HBABO1 } \\
14 \text { COBOO1 } \\
091) A A B 02\end{array}$ & $\begin{array}{l}\text { SCHCFT } \\
\text { VN BUR } \\
\text { WSHTNW }\end{array}$ & $\begin{array}{l}76-06-07 \\
76-06-07 \\
77-07-26 \\
76-00-17 \\
75-05-27\end{array}$ & $\begin{array}{l}.0 \\
.00 \\
.00 \\
.00 \\
.00\end{array}$ & $\begin{array}{l}-0 \\
.00 \\
.00 \\
.00 \\
.00\end{array}$ & $\begin{array}{l}-- \\
0 \\
0 \\
0 \\
0\end{array}$ & $\begin{array}{l}. \overline{0} \\
.00 \\
.00 \\
.00\end{array}$ & $\begin{array}{l}-\overline{0} \\
.00 \\
.00 \\
.00\end{array}$ & $\begin{array}{l}-0 \\
.00 \\
.00 \\
.00 \\
--\end{array}$ & $\begin{array}{l}- \\
.0 \\
.0 \\
.0 \\
.0\end{array}$ & $\begin{array}{l}-- \\
.00 \\
.00 \\
.00 \\
.00\end{array}$ & $\begin{array}{l}.- \\
.00 \\
.00 \\
.00 \\
.00\end{array}$ \\
\hline $\begin{array}{l}03 \mathrm{~S} \\
21 \mathrm{~N}\end{array}$ & $\begin{array}{l}06 E \\
09 W\end{array}$ & $\begin{array}{l}\text { 1 } \mathrm{BHCCO01} \\
04 \mathrm{ABBCO1}\end{array}$ & $\begin{array}{l}\text { WSHTNW } \\
\text { WEXFRD }\end{array}$ & $\begin{array}{l}76-06-15 \\
76-0 b-15 \\
76-06-22\end{array}$ & $\begin{array}{l}.00 \\
.00\end{array}$ & $\begin{array}{l}. \overline{0} \\
.00\end{array}$ & $\begin{array}{r}-\overline{0} \\
0\end{array}$ & $\begin{array}{l}.00 \\
.00\end{array}$ & $\begin{array}{l}-00 \\
.00\end{array}$ & $\begin{array}{l}.00 \\
.00 \\
.00\end{array}$ & $\because 0$ & $\begin{array}{l}.00 \\
.00\end{array}$ & $\begin{array}{l}.00 \\
.00\end{array}$ \\
\hline
\end{tabular}


TABLE 3. WATER-QUALITY DATA. (CONTINUEd)

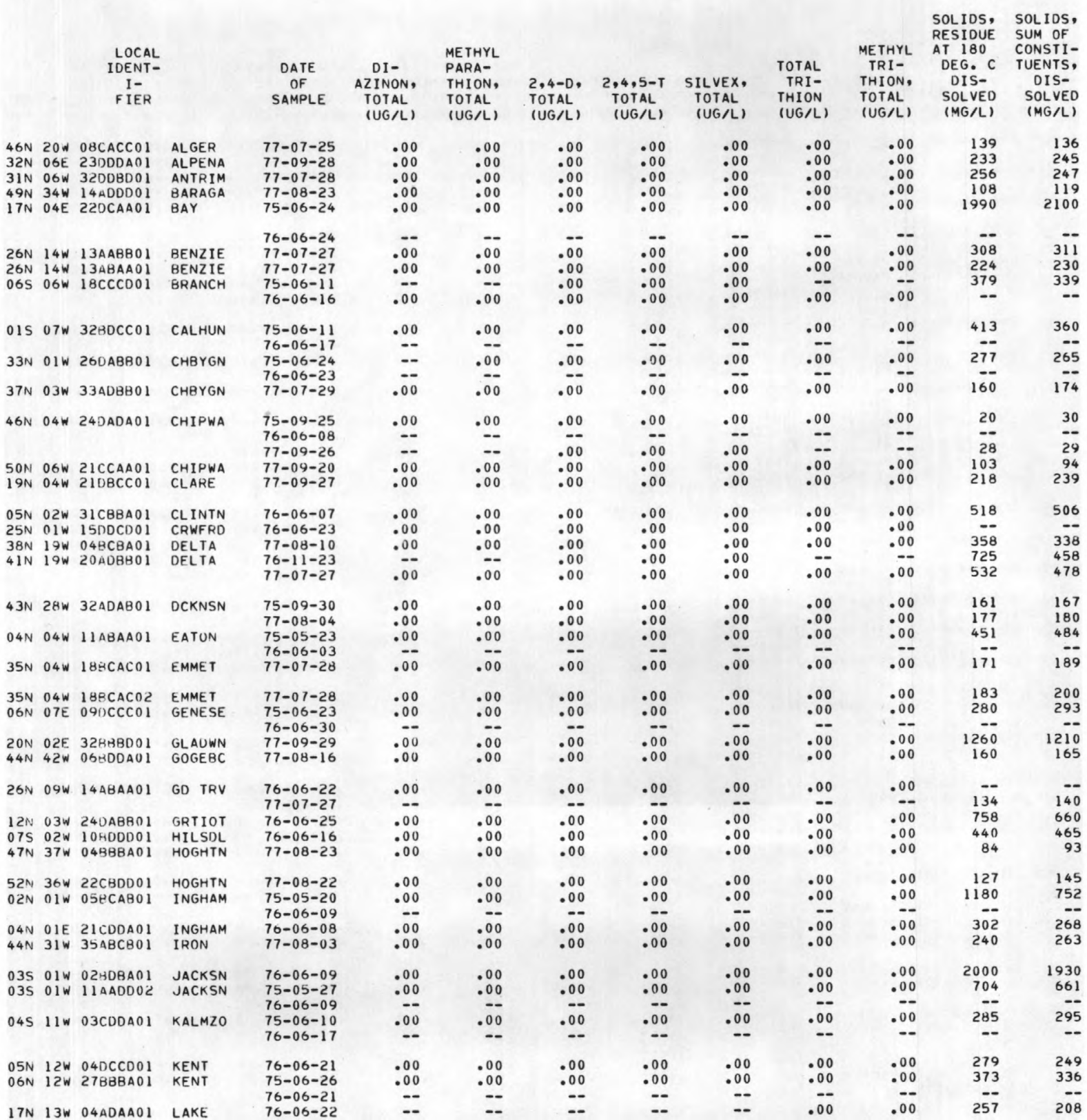


TABLE 3. WATER-QUALITY DATA. (CONTINUEd)

\begin{tabular}{|c|c|c|c|c|c|c|c|c|c|c|c|c|c|}
\hline & & $\begin{array}{l}\text { LOCAL } \\
\text { IOENT- } \\
\text { I- } \\
\text { FIER }\end{array}$ & & $\begin{array}{c}\text { DATE } \\
\text { OF } \\
\text { SAMPLE }\end{array}$ & $\begin{array}{l}\text { DI- } \\
\text { AZINON, } \\
\text { TOTAL } \\
\text { (UGL) }\end{array}$ & $\begin{array}{l}\text { METHYL } \\
\text { PARA- } \\
\text { THION, } \\
\text { TOTAL } \\
\text { (UG/L) }\end{array}$ & $\begin{array}{l}\text { 2,4-D, } \\
\text { TOTAL } \\
\text { (UG/L) }\end{array}$ & $\begin{array}{c}2,4,5-T \\
\text { TOTAL } \\
\text { (UG/L) }\end{array}$ & $\begin{array}{l}\text { SILVEX, } \\
\text { TOTAL } \\
\text { (UG/L) }\end{array}$ & $\begin{array}{l}\text { TOTAL } \\
\text { TRI- } \\
\text { THION } \\
\text { (UG/L) }\end{array}$ & $\begin{array}{l}\text { ME THYL } \\
\text { TRI- } \\
\text { THION, } \\
\text { TOTAL } \\
\text { (UG/L) }\end{array}$ & $\begin{array}{l}\text { SOLIDS, } \\
\text { RESIDUE } \\
\text { AT } 180 \\
\text { DEG. C } \\
\text { DIS- } \\
\text { SOLVED } \\
\text { (MG L ) }\end{array}$ & $\begin{array}{c}\text { SOL IDS, } \\
\text { SUM OF } \\
\text { CONSTI- } \\
\text { TUENTS, } \\
\text { DIS- } \\
\text { SOLVED } \\
\text { (MGIL) }\end{array}$ \\
\hline 065 & $04 E$ & O800BA01 & LENAWE & $\begin{array}{l}75-05-27 \\
76-06-14\end{array}$ & .00 & .00 & .00 & .00 & .00 & .00 & .00 & 522 & 487 \\
\hline $01 \mathrm{~N}$ & $06 E$ & $130 B A B 01$ & LVNGTN & $\begin{array}{l}75-05-22 \\
76-06-10\end{array}$ & .00 & .00 & .00 & .00 & .00 & .00 & .00 & 523 & 437 \\
\hline $49 \mathrm{~N}$ & $12 \mathrm{w}$ & $01 \mathrm{AACB} 01$ & LUCE & $77-07-26$ & .00 & .00 & .00 & .00 & .00 & .00 & .00 & 98 & 101 \\
\hline $\begin{array}{l}42 N \\
43 N \\
44 N\end{array}$ & $\begin{array}{l}02 w \\
09 w \\
26 w\end{array}$ & $\begin{array}{l}07 A A B B 01 \\
1300 D A 01 \\
2 B D A B B 01\end{array}$ & $\begin{array}{l}\text { MACKNC } \\
\text { MACKNC } \\
\text { MARQTE }\end{array}$ & $\begin{array}{l}76-06-09 \\
77-09-27 \\
77-09-22 \\
76-11-22 \\
77-09-29\end{array}$ & $\begin{array}{l}.00 \\
.00 \\
.00 \\
.0 \\
.00\end{array}$ & $\begin{array}{l}.00 \\
.00 \\
.00 \\
-- \\
.00\end{array}$ & $\begin{array}{l}.00 \\
.00 \\
.00 \\
.00 \\
.00\end{array}$ & $\begin{array}{l}.00 \\
.00 \\
.00 \\
.00 \\
.00\end{array}$ & $\begin{array}{l}.00 \\
.00 \\
.00 \\
.00 \\
.00\end{array}$ & $\begin{array}{r}.00 \\
.00 \\
.00 \\
.0 \\
.00\end{array}$ & $\begin{array}{l}.00 \\
.00 \\
.00 \\
.0 \\
.00\end{array}$ & $\begin{array}{l}259 \\
259 \\
222 \\
224 \\
230\end{array}$ & $\begin{array}{l}232 \\
266 \\
217 \\
240 \\
245\end{array}$ \\
\hline $47 \mathrm{~N}$ & $26 \mathrm{w}$ & З०В8DBO 1 & MARQTE & $\begin{array}{l}76-06-01 \\
76-11-03 \\
77-07-18 \\
75-09-23 \\
76-06-01\end{array}$ & $\begin{array}{l}.00 \\
.00 \\
.00 \\
.00 \\
-.\end{array}$ & $\begin{array}{l}.00 \\
.00 \\
.00 \\
.00 \\
.-\end{array}$ & $\begin{array}{l}.00 \\
.00 \\
.00 \\
.00 \\
-.\end{array}$ & $\begin{array}{l}.00 \\
.00 \\
.00 \\
.00 \\
--\end{array}$ & $\begin{array}{l}.00 \\
.00 \\
.00 \\
.00 \\
--\end{array}$ & $\begin{array}{l}.00 \\
.00 \\
.00 \\
.00 \\
.-\end{array}$ & $\begin{array}{l}.00 \\
.00 \\
.00 \\
.00 \\
.-\end{array}$ & $\begin{array}{l}150 \\
141 \\
140 \\
134 \\
--\end{array}$ & $\begin{array}{l}135 \\
140 \\
148 \\
125\end{array}$ \\
\hline $\begin{array}{l}47 \mathrm{~N} \\
18 \mathrm{~N} \\
33 \mathrm{~N}\end{array}$ & $\begin{array}{l}29 \mathrm{w} \\
17 \mathrm{w} \\
27 \mathrm{w}\end{array}$ & $\begin{array}{l}02 \triangle A D D A 01 \\
14 C A B A 01 \\
35 B B C B O 1\end{array}$ & $\begin{array}{l}\text { MARQTE } \\
\text { MASON } \\
\text { MENOME }\end{array}$ & $\begin{array}{l}76-11-04 \\
77-07-20 \\
77-07-20 \\
77-07-26 \\
77-06-30\end{array}$ & $\begin{array}{l}.00 \\
.00 \\
.00 \\
.00 \\
.00\end{array}$ & $\begin{array}{l}.00 \\
.00 \\
.00 \\
.00 \\
.00\end{array}$ & $\begin{array}{l}.00 \\
.00 \\
.00 \\
.00 \\
.00\end{array}$ & $\begin{array}{l}.00 \\
.00 \\
.00 \\
.00 \\
.00\end{array}$ & $\begin{array}{l}.00 \\
.00 \\
.00 \\
.00 \\
.00\end{array}$ & $\begin{array}{l}.00 \\
.00 \\
.00 \\
.00 \\
.00\end{array}$ & $\begin{array}{l}.00 \\
.00 \\
.00 \\
.00 \\
.00\end{array}$ & $\begin{array}{r}120 \\
117 \\
97 \\
194 \\
564\end{array}$ & $\begin{array}{r}115 \\
124 \\
87 \\
199 \\
526\end{array}$ \\
\hline $37 \mathrm{~N}$ & $26 w$ & $190 A D A 01$ & MENOME & $\begin{array}{l}76-11-24 \\
77-06-29\end{array}$ & $\begin{array}{l}.00 \\
.00\end{array}$ & $\begin{array}{l}.00 \\
.00\end{array}$ & $\begin{array}{r}.00 \\
.00\end{array}$ & $\begin{array}{r}.00 \\
.00\end{array}$ & $\begin{array}{l}.00 \\
.00\end{array}$ & $\begin{array}{l}.00 \\
.00\end{array}$ & $\begin{array}{l}.00 \\
.00\end{array}$ & $\begin{array}{l}314 \\
370\end{array}$ & $\begin{array}{l}321 \\
356\end{array}$ \\
\hline $\begin{array}{l}23 \mathrm{~N} \\
31 \mathrm{~N}\end{array}$ & $\begin{array}{l}05 \mathrm{~W} \\
04 \mathrm{E}\end{array}$ & $\begin{array}{l}35 \text { CABA01 } \\
05 \text { CBDB } 1\end{array}$ & $\begin{array}{l}\text { MSAUKE } \\
\text { MNTMRY }\end{array}$ & $\begin{array}{l}77-07-29 \\
77-09-28\end{array}$ & $\begin{array}{l}.00 \\
.00\end{array}$ & $\begin{array}{l}.00 \\
.00\end{array}$ & $\begin{array}{l}.00 \\
.00\end{array}$ & $\begin{array}{l}.00 \\
.00\end{array}$ & $\begin{array}{r}.00 \\
.00\end{array}$ & $\begin{array}{l}.00 \\
.00\end{array}$ & $\begin{array}{l}.00 \\
.00\end{array}$ & $\begin{array}{l}206 \\
159\end{array}$ & $\begin{array}{l}218 \\
169\end{array}$ \\
\hline $11 \mathrm{~N}$ & $15 \mathrm{w}$ & $34 \triangle 0 D D 01$ & MUSKGN & $76-06-28$ & -- & - & -- & -- & -- & -- & -- & 1140 & 968 \\
\hline $02 \mathrm{~N}$ & $08 E$ & 18UBADO1 & OAKLNO & $\begin{array}{l}75-05-22 \\
76-06-10\end{array}$ & .00 & .00 & .00 & .00 & .00 & .00 & .00 & $\begin{array}{r}370 \\
--\end{array}$ & $\begin{array}{l}378 \\
--\end{array}$ \\
\hline $03 \mathrm{~N}$ & $08 \mathrm{E}$ & $0.31) 8 \Delta B 01$ & OAKLND & $\begin{array}{l}75-05-22 \\
76-06-10\end{array}$ & .00 & .00 & .00 & .00 & .00 & .00 & .00 & 274 & 284 \\
\hline $23 / \mathrm{N}$ & $01 E$ & ОZВАAA0Z & OGEMAW & $76-06-24$ & .00 & .00 & .00 & .00 & .00 & .00 & .00 & -- & -- \\
\hline $\begin{array}{l}46 \mathrm{~N} \\
48 \mathrm{~N} \\
51 \mathrm{~N}\end{array}$ & $\begin{array}{l}38 w \\
43 w \\
41 w\end{array}$ & $\begin{array}{l}30 \triangle D D D 01 \\
13 C D D A 01 \\
0 B \div D B C 01\end{array}$ & $\begin{array}{l}\text { ONTNGN } \\
\text { ONTNGN } \\
\text { ONTNGN }\end{array}$ & $\begin{array}{l}77-09-29 \\
76-06-14 \\
77-08-18 \\
75-09-22 \\
76-06-15\end{array}$ & $\begin{array}{l}.0 \\
.00 \\
.00 \\
.00 \\
--\end{array}$ & $\begin{array}{l}.00 \\
.00 \\
.00 \\
.00 \\
--\end{array}$ & $\begin{array}{l}.- \\
.00 \\
.00 \\
.00 \\
--\end{array}$ & $\begin{array}{l}-\cdot \\
.00 \\
.00 \\
.00 \\
--\end{array}$ & $\begin{array}{l}-\overline{0} \\
.00 \\
.00 \\
.00 \\
--\end{array}$ & $\begin{array}{l}.0 \\
.00 \\
.00 \\
.00 \\
--\end{array}$ & $\begin{array}{l}.0 \\
.00 \\
.00 \\
.00 \\
--\end{array}$ & $\begin{array}{r}192 \\
110 \\
311 \\
310 \\
--\end{array}$ & $\begin{array}{l}197 \\
109 \\
285 \\
304\end{array}$ \\
\hline $\begin{array}{l}31 \mathrm{~N} \\
33 \mathrm{~N}\end{array}$ & $\begin{array}{l}\text { O3W } \\
\text { OSE }\end{array}$ & $\begin{array}{l}34 E C D D 01 \\
\text { OHABBSOI }\end{array}$ & $\begin{array}{l}\text { OISEGO } \\
\text { PRSO I }\end{array}$ & $\begin{array}{l}77-07-28 \\
75-06-24\end{array}$ & $\begin{array}{l}.00 \\
.00\end{array}$ & $\begin{array}{l}.00 \\
.00\end{array}$ & $\begin{array}{l}.00 \\
.00\end{array}$ & .00 & $\begin{array}{l}.00 \\
.00\end{array}$ & $\begin{array}{l}.00 \\
.00\end{array}$ & $\begin{array}{l}.00 \\
.00\end{array}$ & $\begin{array}{l}260 \\
340\end{array}$ & $\begin{array}{l}257 \\
324\end{array}$ \\
\hline $24 \mathrm{~N}$ & $02 \mathrm{w}$ & $20 \mathrm{HARAOI}$ & ROSCMN & $\begin{array}{l}76-06-23 \\
75-06-25 \\
76-06-24\end{array}$ & .00 & .00 & .00 & .0 & .00 & .00 & .00 & 183 & $1 \overline{90}$ \\
\hline $12 \mathrm{~N}$ & $13 \mathrm{E}$ & 33000001 & SANLAC & $\begin{array}{l}75-06-23 \\
76-06-29\end{array}$ & .00 & .00 & .00 & .00 & .00 & .00 & .00 & 362 & 358 \\
\hline $\begin{array}{l}13 \mathrm{~N} \\
41 \mathrm{~N}\end{array}$ & $\begin{array}{l}13 E \\
16 W\end{array}$ & $\begin{array}{l}12 \triangle D A A 01 \\
06 C D C B 01\end{array}$ & $\begin{array}{l}\text { SANLAC } \\
\text { SCHCFT }\end{array}$ & $\begin{array}{l}76-06 \\
77-08\end{array}$ & $\begin{array}{r}.00 \\
.00\end{array}$ & $\begin{array}{r}.00 \\
.00\end{array}$ & $\begin{array}{l}.00 \\
.00\end{array}$ & $\begin{array}{r}.00 \\
.00\end{array}$ & $\begin{array}{l}.00 \\
.00\end{array}$ & $\begin{array}{l}.00 \\
.00\end{array}$ & $\begin{array}{l}.00 \\
.00\end{array}$ & 230 & 239 \\
\hline $45 \mathrm{~N}$ & $13 \mathrm{w}$ & $10 \mathrm{CCCB} 01$ & SCHCF T & $75-09-24$ & .00 & .00 & 1.1 & .25 & .00 & .00 & .00 & 329 & 328 \\
\hline $47 N$ & 1 & ЗоसВBВ01 & SCHCF T & $\begin{array}{l}76-06-07 \\
76-06-07 \\
77-07-26\end{array}$ & $\begin{array}{l}.- \\
.00 \\
.00\end{array}$ & $\begin{array}{l}. \overline{0} \\
.00\end{array}$ & $\begin{array}{l}.00 \\
.00 \\
.00\end{array}$ & $\begin{array}{l}.00 \\
.00 \\
.00\end{array}$ & .00 & $\begin{array}{l}.0 \\
.00 \\
.00\end{array}$ & $\begin{array}{l}.0 \\
.00 \\
.00\end{array}$ & $\begin{array}{l}178 \\
156\end{array}$ & $\begin{array}{l}176 \\
167\end{array}$ \\
\hline $\begin{array}{l}04 \mathrm{~S} \\
02 \mathrm{~S}\end{array}$ & $\begin{array}{l}16 \mathrm{~W} \\
03 \mathrm{E}\end{array}$ & $\begin{array}{l}14 C D A D 01 \\
091, A A B 02\end{array}$ & $\begin{array}{l}\text { VN BUR } \\
\text { WSHTNW }\end{array}$ & $\begin{array}{l}76-06-17 \\
75-05-27\end{array}$ & $\begin{array}{l}.00 \\
.00\end{array}$ & $\begin{array}{l}.00 \\
.00\end{array}$ & $\begin{array}{l}.00 \\
.00\end{array}$ & $\begin{array}{l}.00 \\
.00\end{array}$ & $\begin{array}{l}.00 \\
.00\end{array}$ & $\begin{array}{l}.00 \\
.00\end{array}$ & $\begin{array}{l}.00 \\
.00\end{array}$ & $\begin{array}{l}267 \\
177\end{array}$ & $\overline{171}$ \\
\hline $\begin{array}{l}03 \mathrm{~S} \\
21 \mathrm{~N}\end{array}$ & $\begin{array}{l}06 \mathrm{E} \\
09 \mathrm{~W}\end{array}$ & $\begin{array}{l}16 A C C D 01 \\
04 \triangle B B C 01\end{array}$ & $\begin{array}{l}\text { WSHTNW } \\
\text { WEXFRD }\end{array}$ & $\begin{array}{l}76-06-15 \\
76-06-15 \\
76-06-22\end{array}$ & .00 & .00 & $\begin{array}{l}=- \\
=-\end{array}$ & $=$ & $=-$ & $\begin{array}{l}. \overline{0} \\
.00\end{array}$ & $\begin{array}{l}.0 \\
.00 \\
.00\end{array}$ & $\begin{array}{r}1130 \\
359\end{array}$ & $\begin{array}{l}917 \\
316\end{array}$ \\
\hline
\end{tabular}


TABLE 3. WATER-QUALITY DATA. (CONTINUED)

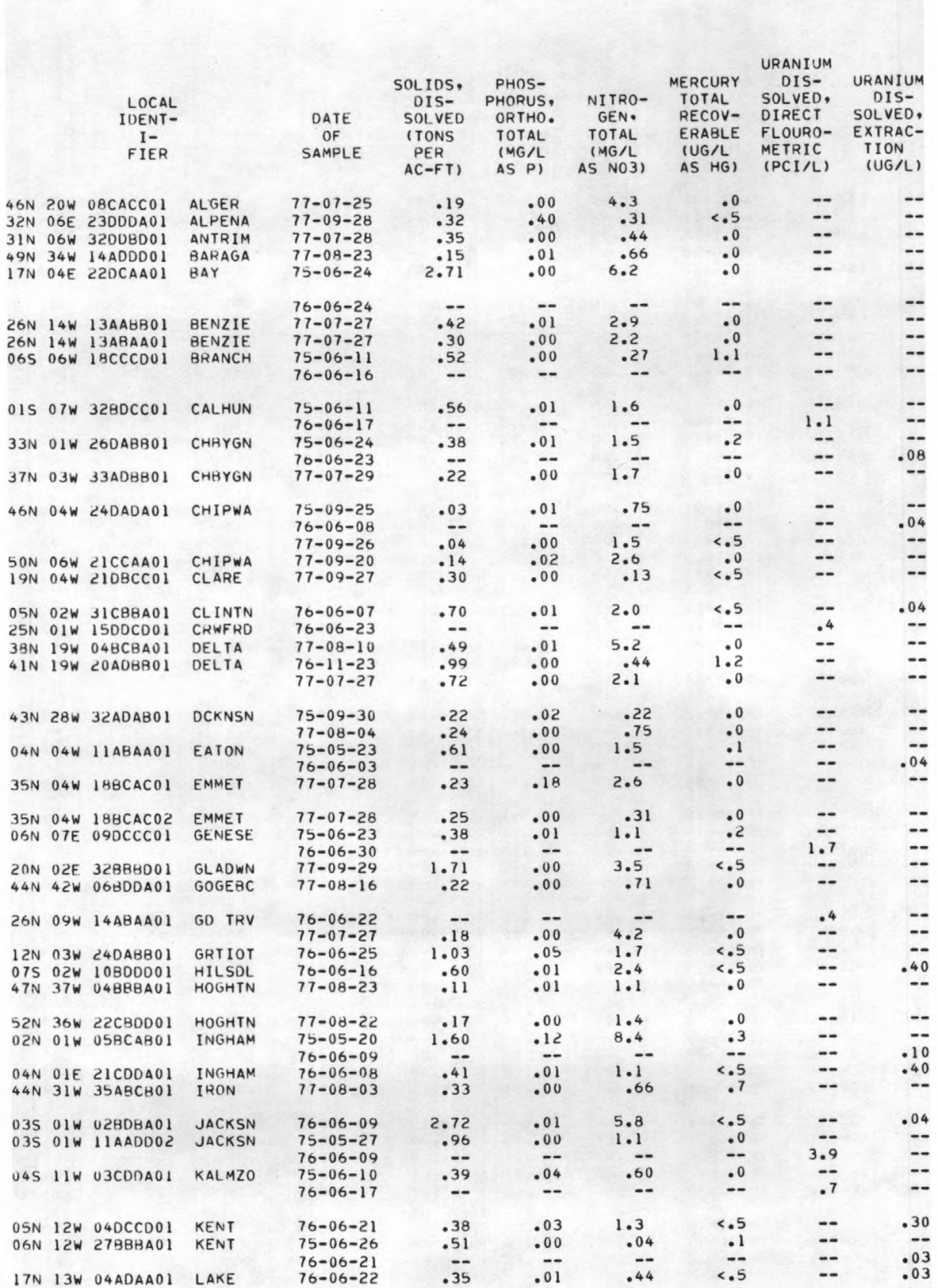


TABLE 3. WATER-QUALITY DATA. (CONTINUED)

\begin{tabular}{|c|c|c|c|c|c|c|c|c|c|c|}
\hline & & $\begin{array}{l}\text { LOCAL } \\
\text { IDENT- } \\
\text { I- } \\
\text { FIER }\end{array}$ & & $\begin{array}{c}\text { DATE } \\
\text { OF } \\
\text { SAMPLE }\end{array}$ & $\begin{array}{l}\text { SOL IOS, } \\
\text { DIS- } \\
\text { SOLVED } \\
\text { (TONS } \\
\text { PER } \\
\text { AC-FT) }\end{array}$ & $\begin{array}{l}\text { PHOS- } \\
\text { PHORUS, } \\
\text { ORTHO. } \\
\text { TOTAL } \\
\text { (MG/L } \\
\text { AS P) }\end{array}$ & $\begin{array}{l}\text { NITRO- } \\
\text { GEN, } \\
\text { TOTAL } \\
\text { (MG/L } \\
\text { AS NO3) }\end{array}$ & $\begin{array}{l}\text { MERCURY } \\
\text { TOTAL } \\
\text { RECOV- } \\
\text { ERABLE } \\
\text { (UG/L } \\
\text { AS HG) }\end{array}$ & $\begin{array}{l}\text { URANIUM } \\
\text { DIS- } \\
\text { SOLVED, } \\
\text { OIRECT } \\
\text { FLOURO- } \\
\text { METRIC } \\
\text { (PCI/L) }\end{array}$ & $\begin{array}{l}\text { URANIUM } \\
\text { DIS- } \\
\text { SOLVED, } \\
\text { EXTRAC- } \\
\text { TION } \\
\text { (UGL) }\end{array}$ \\
\hline $06 \mathrm{~S}$ & $04 E$ & U8DOBAOI & LENAWE & $\begin{array}{l}75-05-27 \\
76-06-14\end{array}$ & .71 & .00 & 2.9 & .0 & $=$ &.$\overline{10}$ \\
\hline $01 \mathrm{~N}$ & $06 E$ & $130 B A B 01$ & LVNGTN & $\begin{array}{l}75-05-22 \\
76-06-10\end{array}$ & .71 & .00 & .18 & $\therefore$ & $=$ & $1.4^{--}$ \\
\hline $49 \mathrm{~N}$ & $12 W$ & O1BACBO1 & LUCE & $77-07-26$ & .13 & .00 & 1.9 & .0 & -- & -- \\
\hline $42 \mathrm{~N}$ & $02 W$ & 07AABBOI & MACKNC & $\begin{array}{l}76-06-09 \\
77-09-27\end{array}$ & $\begin{array}{l}.35 \\
.35\end{array}$ & $\begin{array}{l}.01 \\
.00\end{array}$ & $\begin{array}{l}1.6 \\
3.1\end{array}$ & $<.5$ & $\therefore$ & $=$ \\
\hline $43 \mathrm{~N}$ & $09 \mathrm{~W}$ & $13000 A 01$ & MACKNC & $77-09-22$ & .30 & .01 & 1.1 & .0 & -- & -- \\
\hline $44 \mathrm{~N}$ & $26 \mathrm{~W}$ & $28 D A B B 01$ & MARQTE & $\begin{array}{l}76-11-22 \\
77-09-29\end{array}$ & $\begin{array}{r}.30 \\
.31\end{array}$ & $\begin{array}{l}.00 \\
.00\end{array}$ & .31 & $\begin{array}{l}<.5 \\
<.5\end{array}$ & $=-$ & $=$ \\
\hline $47 \mathrm{~N}$ & $26 \mathrm{~W}$ & 36880801 & MARQTE & $\begin{array}{l}76-06-01 \\
76-11-03\end{array}$ & $\begin{array}{l}.20 \\
.19\end{array}$ & $\begin{array}{l}.02 \\
.01\end{array}$ & $\begin{array}{l}1.4 \\
.97\end{array}$ & $\begin{array}{l}<.5 \\
1.1\end{array}$ & $=-$ & $=-$ \\
\hline & & & & $77-07-18$ & .19 & .01 & 1.4 & .0 & $=-$ & $=$ \\
\hline $47 \mathrm{~N}$ & $28 \mathrm{~W}$ & $03 \mathrm{CCDC} 01$ & MARQTE & $\begin{array}{l}75-09-23 \\
76-06-01\end{array}$ & .18 & .10 & 2.4 & -3 & $=$ &.$\overline{8}$ \\
\hline & & & & $\begin{array}{l}76-11-04 \\
77-07-20\end{array}$ & $\begin{array}{l}.16 \\
.16\end{array}$ & $\begin{array}{l}.04 \\
.08\end{array}$ & $\begin{array}{l}2.7 \\
2.0\end{array}$ & $\begin{array}{l}<.5 \\
2.1\end{array}$ & $=$ & $=-$ \\
\hline $47 \mathrm{~N}$ & $29 \mathrm{~W}$ & O2ADOA01 & MARQTE & $77-07-20$ & .13 & .07 & 1.2 & .0 & -- & - \\
\hline $18 \mathrm{~N}$ & $17 \mathrm{~W}$ & 14CABAO1 & MASON & $77-07-26$ & .26 & .02 & 2.7 & 0 & $=$ & $=-$ \\
\hline $33 N$ & $27 \mathrm{~W}$ & $358 B C 801$ & MENOME & $77-06-30$ & .77 & .00 & 1.2 & .0 & -- & - \\
\hline $37 \mathrm{~N}$ & $26 \mathrm{~W}$ & 190AUA0I & MENOME & $\begin{array}{l}76-11-24 \\
77-06-29\end{array}$ & $\begin{array}{l}.43 \\
.50\end{array}$ & $\begin{array}{l}.01 \\
.00\end{array}$ & $\begin{array}{l}.93 \\
1.2\end{array}$ & $\begin{array}{r}<.5 \\
.0\end{array}$ & $=$ & $=$ \\
\hline $23 \mathrm{~N}$ & OSW & 35CABA0 1 & MSAUKE & $77-07-29$ & .28 & .01 & 1.9 & .0 & -- & -- \\
\hline $31 \mathrm{~N}$ & $04 \mathrm{~F}$ & 05 CBOB01 & MNTMRY & $77-09-28$ & .22 & .00 & .89 & $<.5$ & $=$ & $=$ \\
\hline $11 \mathrm{~N}$ & $15 \mathrm{~W}$ & 34ADUD01 & MUSKGN & $76-06-28$ & 1.55 & .18 & 34 & -- & -- & -- \\
\hline $02 N$ & $08 E$ & $180 B A 001$ & OAKLND & $75-05-22$ & .50 & .00 & .89 & .1 & -- & -- \\
\hline & & & & $76-06-10$ & -- & -- & -- & -- & - & - 30 \\
\hline $03 \mathrm{~N}$ & OBE & ОЗОВАВО1 & OAKLND & $75-05-22$ & .37 & .01 & 1.2 & $\cdot 0$ & - & $\overline{10}$ \\
\hline $23 N$ & $01 E$ & О2ВАAA0Z & OGEMAW & $\begin{array}{l}76-06-10 \\
76-06-24\end{array}$ & $=$ & $=$ & $=$ & $=$ & 1.0 & -10 \\
\hline & & & & $77-09-29$ & .26 & .00 & 2.0 & $<.5$ & -- & $-\overline{-}$ \\
\hline $46 \mathrm{~N}$ & $38 \mathrm{~W}$ & $30 A D D 001$ & ONTNGN & $76-06$ & .15 & .01 & .75 & $<.5$ & -- & .20 \\
\hline $4 \mathrm{BN}$ & $43 w$ & $13 C D D A 01$ & ONTINGN & $77-08-18$ & .42 & .01 & 1.2 & .0 & -- & $=-$ \\
\hline $51 N$ & $41 \mathrm{~W}$ & $08 B D B C 01$ & ONTNGN & $\begin{array}{l}75-09-22 \\
76-06-15\end{array}$ & .42 & .11 & $\stackrel{49}{--}$ & -3 & $4 . \overline{3}$ & $=$ \\
\hline $31 \mathrm{~N}$ & $03 w$ & $34 \mathrm{BCDO} 01$ & OTSEGO & $77-07-28$ & .35 & .00 & $4 \cdot 9$ & .0 & -- & -- \\
\hline $33 \mathrm{~N}$ & $06 E$ & О8в8в801 & PRSQ I & $\begin{array}{l}75-06-24 \\
76-06-23\end{array}$ & .46 & .01 & ${ }^{27}--$ & -0 &.$\overline{9}$ & $=$ \\
\hline $24 \mathrm{~N}$ & $02 w$ & СОВАВАО 1 & ROSCMN & $\begin{array}{l}75-06-25 \\
76-06-24\end{array}$ & .25 & .01 & 1.2 & $\therefore$ & $=$ & $=$ \\
\hline $12 N$ & $13 E$ & 33000001 & SANLAC & $\begin{array}{l}75-06-23 \\
70-06-29\end{array}$ & .49 & .01 & .71 & .0 & .9 & $=$ \\
\hline $13 \mathrm{~N}$ & $13 E$ & $12 A D A A 01$ & SANLAC & $70-06-29$ & .31 & .03 & 1.2 & $<.5$ & $=$ & $=-$ \\
\hline $\begin{array}{l}41 \mathrm{~N} \\
45 \mathrm{~N}\end{array}$ & $16 \mathrm{~W}$ & $\begin{array}{l}\text { U6CDCB01 } \\
16 C C C B 01\end{array}$ & $\begin{array}{l}\text { SCHCFT } \\
\text { SCHCFT }\end{array}$ & $\begin{array}{l}77-08-09 \\
75-09-24\end{array}$ &.$\overline{45}$ &.$\overline{00}$ &.$\overline{93}$ &.$\overline{4}$ & $=$ & $=$ \\
\hline & & DOCLCBOL & Scher & & & & & & & \\
\hline $7 \mathrm{~N}$ & $16 \mathrm{~W}$ & ЗовВВВ01 & & $\begin{array}{l}76- \\
76-\end{array}$ & .24 & .01 & $4 . \overline{3}$ & $<.5$ & $\overline{-}$ &.$\overline{06}$ \\
\hline & & 30800801 & sent? & $77-07-26$ & .21 & .00 & 4.1 & .2 & -- & -- \\
\hline 045 & $16 \mathrm{w}$ & $14 \mathrm{CDHOO} 1$ & VN BUR & $76-06-17$ & .36 & .0 & $2 \cdot 0$ & $<.5$ & $<.4$ & -- \\
\hline 025 & $03 E$ & 09DAAB02 & WSHTNW & $75-05-27$ & .24 & .00 & .66 & .0 & - & - \\
\hline & & & & $76-0$ & $\overline{54}$ & $\overline{0}$ & - & $<. \overline{5}$ & .5 & .04 \\
\hline 35 & OOE & 16BCCD01 & WSHTNW & & 1.54 & -00 & $\begin{array}{l}2.1 \\
1.3\end{array}$ & $\begin{array}{l}<.5 \\
<.5\end{array}$ & 1.5 & - \\
\hline in & $09 \mathrm{w}$ & $04 \mathrm{ABBC} 01$ & WEXFRO & $70-06-22$ & .49 & .00 & 1.3 & .3 & & - \\
\hline
\end{tabular}




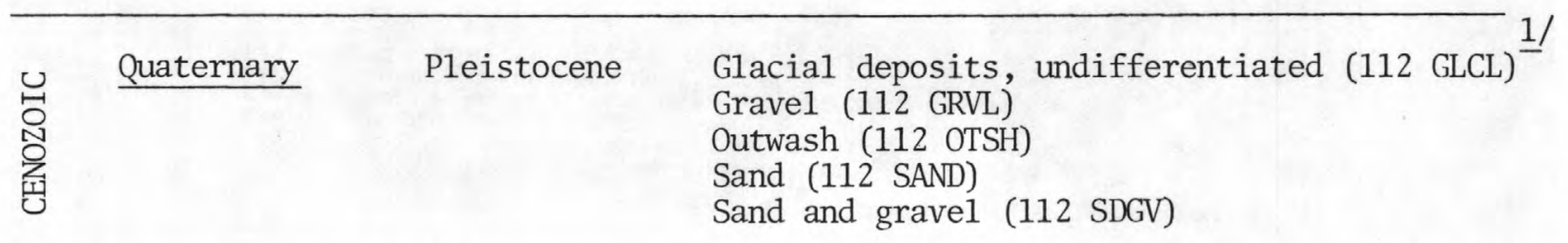

\begin{tabular}{|c|c|c|c|}
\hline \multirow{8}{*}{ 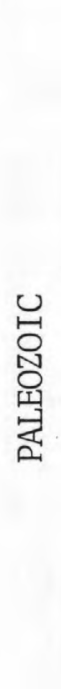 } & $\underline{\text { Pennsylvanian }}$ & Middle & Saginaw Formation (324 SGNW) \\
\hline & $\underline{\text { Mississippian }}$ & Lower & Marsha11 Formation (337 MRSL) \\
\hline & $\underline{\text { Devonian }}$ & Upper & Traverse Group (341 TRVR) \\
\hline & $\underline{\text { Silurian }}$ & Middle & $\begin{array}{l}\text { Engadine Dolomite ( } 355 \text { ENGD) } \\
\text { Manistique Dolomite ( } 355 \text { MNSQ) } \\
\text { Burnt Bluff Formation ( } 355 \text { BBLF) }\end{array}$ \\
\hline & $\underline{\text { Ordovician }}$ & Upper & Richmondian Stage (362 RCMD) \\
\hline & & Middle & $\begin{array}{l}\text { B1ack River Formation ( } 365 \text { BKRV) } \\
\text { Trenton-Black River Group ( } 365 \text { TBRV) }\end{array}$ \\
\hline & & Canadian & Prairie Du Chien Group (368 PRDC) \\
\hline & Cambrian & Upper & Lake Superior Sandstone (372 LKSP) \\
\hline 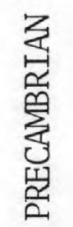 & $\underline{\text { Proterozoic }}$ & $\mathrm{Y}$ & $\begin{array}{l}\text { Jacobsville Sandstone ( } 420 \text { JCBV) } \\
\text { Freda Sandstone ( } 420 \text { FRED) }\end{array}$ \\
\hline
\end{tabular}

1/ Rock units in this report are identified by an alpha-numeric code. This code is used to standardize rock-unit identification and is based on the stratigraphic coding system proposed by the American Association of Petroleum Geologists (Cohee, 1967). The code used in this report consists of two parts. The first 3 characters are numeric and identify the age of the unit. For example, in the code 324 SGNW, the " 3 " is a broad classification that identifies the unit as being Paleozoic, the " 2 " narrows the classification and relates the unit to the Pennsylvanian System, and the "4" further restricts the age and relates the unit to the Middle Series. The first four alpha characters identify the name of the rock unit. The alpha code is developed by eliminating letters from the original name until only 4 remain (Burk, 1966). For example, in the code given above the "SGNW" identifies the unit as the Saginaw Formation. If it is necessary to modify the rock-unit name, an eighth character--either alpha or numeric--is added. 


\section{SELECTED REFERENCES}

\section{Publications of the U.S. Geological Survey}

Water-Supply Papers

Allen, W. B., Miller, J. B., and Wood, W. W., 1972, Availability of water in Kalamazoo County, Michigan: U.S. Geological Survey Water-Supply Paper 1973, 129 p., 36 figs., 9 pls.

Deutsch, Morris, 1963, Ground-water contamination and legal controls in Michigan: U.S. Geological Survey Water-Supply Paper 1691, 79 p., 23 figs.

Lohr, E. W., and Love, S. K., 1954, The industrial utility of public water supplies in the United States 1952, pt. 1, States east of the Mississippi River: U.S. Geological Survey Water-Supply Paper 1299, 639 p., 3 figs., 5 pls.

McGuinness, C. L., 1963, The role of ground water in the national water situation: U.S. Geological Survey Water-Supply Paper 1800, 1121 p., 2 figs., 4 pls.

McGuinness, C. L., Poindexter, O. F., and Otten, E. G., 1949, Ground-water supplies of the Ypsilanti area, Michigan: U.S. Geological Survey Water-Supply Paper 1078, 105 p., 7 figs., 5 pls.

Reed, J. E., Deutsch, Morris, and Wiitala, S. W., 1966, Induced recharge of an artesian glacial-drift aquifer at Kalamazoo, Michigan: U.S. Geological Survey Water-Supply Paper 1594-D, 62 p., 36 figs., 2 pls.

Twenter, F. R., and Knutilla, R. L., 1972, Water for a rapidly growing urban community--Oakland County, Michigan: U.S. Geological Survey Water-Supply Paper 2000,150 p., 90 figs.

Vanlier, K. E., 1963, Ground-water resources of the Alma area, Michigan: U.S. Geological Survey Water-Supply Paper 1619-E, 66 p., 20 figs., 2 p1s.

Vanlier, K. E., Wood, W. W., and Brunett, J. O., 1973, Water-supply development and management alternatives for Clinton, Eaton, and Ingham Counties, Michigan: U.S. Geological Survey Water-Supply Paper 1969, 111 p., 35 figs., 3 pls.

Wiitala, S. W., Newport, T. G., and Skinner, E. L., 1967, Water resources of the Marquette Iron Range area, Michigan: U.S. Geological Survey WaterSupply Paper 1842, 142 p., 40 figs., 4 pls.

Wiitala, S. W., Vanlier, K. E., and Krieger, R. A., 1963, Water resources of the Flint area, Michigan: U.S. Geological Survey Water-Supply Paper 1499-E, 86 p., 32 figs., 6 pls. 
Water-Supply Papers (contain ground-water data for Michigan)

\begin{tabular}{|c|c|c|c|c|c|}
\hline Year & $\begin{array}{c}\text { WSP } \\
\text { Number } \\
\end{array}$ & Year & $\begin{array}{c}\text { WSP } \\
\text { Number } \\
\end{array}$ & Year & $\begin{array}{l}\text { WSP } \\
\text { Number } \\
\end{array}$ \\
\hline 193 & 777 & 1944 & 1016 & 1953 & 1265 \\
\hline 193 & 817 & 1945 & 1023 & 1954 & 1321 \\
\hline 1937 & 840 & 1946 & 1071 & 1955 & 1404 \\
\hline 1938 & 845 & 1947 & 1096 & $1956-57$ & 1537 \\
\hline 1939 & 886 & 1948 & 1126 & $1958-62$ & 1782 \\
\hline 1940 & 906 & 1949 & 1156 & $1963-67$ & 1977 \\
\hline 1941 & 936 & 1950 & 1165 & $1968-72$ & 2140 \\
\hline 19 & 944 & 1951 & 1191 & & \\
\hline & 986 & 1952 & 1221 & & \\
\hline
\end{tabular}

\section{Circulars}

Murray, C. R., and Reeves, E. B., 1977, Estimated use of water in the United States, 1975: U.S. Geological Survey Circular 765, 39 p., 12 figs.

Strame1, G. J., Wis1er, C. 0., and Laird, L. B., 1954, Water resources of the Grand Rapids area, Michigan: U.S. Geological Survey Circular 323, 40 p., 29 figs., 3 pls.

Wisler, C. O., Strame1, G. J., and Laird, L. B., 1952, Water resources of the Detroit area, Michigan: U.S. Geological Survey Circular 183, 36 p., 30 figs., 4 pls.

Water-Data Reports

\begin{tabular}{|c|c|}
\hline Year & WDR Number \\
\hline 1975 & MI - 75-1 \\
\hline 1976 & MI- $76-1$ \\
\hline 1977 & MI-77-1 \\
\hline
\end{tabular}

Other Publications

Allen, W. B., 1977, Flowing wells in Michigan, 1974: Michigan Geological Survey Water Information Series Report 2, 27 p., 5 figs., 2 pls., 16 refs.

Borton, T. E., 1974, Planning perspectives on water resources, Washtenaw County, Michigan: Washtenaw County Metropolitan Planning Commission, $69 \mathrm{p}$. 
Brown, E. A., and Stuart, W. T., 1951, Ground-water resources of the glacial deposits in the Bessemer area, Michigan, 1950: Michigan Geological Survey Progress Report 14, 68 p., 27 figs.

Deutsch, Morris, 1956, Effects of dissemination of radioactive materials on water resources conservation--with special references to Michigan: Michigan State University Agricultural Experiment Station Water Bulletin 2.

---- 1961a, Hydrogeologic aspects of ground-water pollution: Water We11 Journa1, v. 15 , no. 9.

-.-- 1961b, Incidents of chromium contamination of ground-water in Michigan: U.S. Public Health Service Technical Report W61-5, p. 98-104.

--.- 1962a, Controlled induced-recharge tests at Kalamazoo, Michigan: Journal of American Water Works Association, v. 54, no. 2, p. 181-196.

----- 1962b, Phenol contamination of an artesian aquifer at Alma, Michigan: Proceedings of the Society for Water Treatment and Examination, v. 11, p. 94-100, 2 figs.

Deutsch, Morris, Burt, E. M., and Vanlier, K. E., 1958, Summary of groundwater investigations in the Holland area, Michigan: Michigan Geological Survey Progress Report 20, 87 p., 16 figs.

Deutsch, Morris, Vanlier, K. E., and Giroux, P. R., 1960, Ground-water hydrology and glacial geology of the Kalamazoo area, Michigan: Michigan Geological Survey Progress Report 23, 122 p., 21 figs.

Deutsch, Morris, and Vanlier, K. E., 1961, Ground water for Michigan's future: U.S. Geological Survey open-file report.

Doonan, C. J., and Byerlay, J. R., 1973, Ground water and geology of Baraga County, Michigan: Michigan Geological Survey Water Investigation 11, 26 p., 2 figs., 2 pls.

Doonan, C. J., and Hendrickson, G. E., 1967, Ground water in Iron County, Michigan: Michigan Geological Survey Water Investigation 7, 61 p., 3 figs., 2 pls.

---- 1968a, Ground water in Gogebic County, Michigan: Michigan Geological Survey Water Investigation 8, 22 p., 5 figs., 2 p1s.

---- 1969, Ground water in Ontonagon County, Michigan: Michigan Geological Survey Water Investigation 9, 29 p., 5 figs., 1 pl.

Doonan, C. J., Hendrickson, G. E., and Byerlay, J. R., 1970, Ground water and geology of Keweenaw Peninsula, Michigan: Michigan Geological Survey Water Investigation 10,41 p., 1 fig., 2 pls. 
Mozola, A. J., 1970, Geology for environmental planning in Monroe County, Michigan: Michigan Geological Survey Report Investigation 13, 34 p., 18 figs., 6 pls.

Sinclair, W. C., 1959, Reconnaissance of the ground-water resources of Schoolcraft County, Michigan: Michigan Geological Survey Progress Report 22, 84 p., 14 figs.

Sinclair, W. C., 1960, Reconnaissance of the ground-water resources of Delta County, Michigan: Michigan Geological Survey Progress Report 24, 93 p., 13 figs.

Stuart, W. T., 1945, Ground-water resources of the Lansing area, Michigan: Michigan Geological Survey Progress Report 13, 35 p., 11 figs.

Stuart, W. T., Brown, E. A., and Rhodehame1, E. C., 1954, Ground-water investigations of the Marquette iron-mining district, Michigan: Michigan Geological Survey Technical Report 3, 92 p., 8 figs.

Stuart, W. T., and Stallman, R. W., 1945, Ground-water resources of the Benton Harbor area, Michigan: Michigan Geological Survey Progress Report 12, 15 p., 4 figs.

Stuart, W. T., Theis, C. V., and Stanley, G. M., 1948, Ground-water problems in the Iron River district, Michigan: Michigan Geological Survey Technical Report 2, 59 p., 16 figs.

Terwilliger, F. W., 1954, The glacial geology and ground-water resources of Van Buren County, Michigan pt. 1 of Occasional papers for 1954 on the geology of Michigan: Michigan Geological Survey Publication 48, p. 1-95, 24 figs., 1 p1.

Twenter, F. R., 1975, Ground water and geology--Southeastern Michigan: U.S. Army Corps of Engineers, Detroit; Michigan, 143 p., 31 figs., 36 tables, 46 refs.

Twenter, F. R., Knutilla, R. L., and Now1in, J. O., 1976, Water resources of Washtenaw County, Michigan: Washtenaw County Planning Commission, 143 p., 43 figs., 18 tables, 25 refs.

Vanlier, K. E., 1959, Reconnaissance of the ground-water resources of Luce County, Michigan: Michigan Geological Survey Progress Report 21, 76 p., 11 figs., 3 pls.

---- 1962, Summary of ground-water investigations in the Elsie area, Michigan: Michigan Geological Survey Progress Report 25, 35 p., 7 figs.

---- 1963a, Ground water in Alger County, Michigan: Michigan Geological Survey Water Investigation 1, 55 p., 13 figs. 
Vanlier, K. E., 1963b, Ground water in Menominee County, Michigan: Michigan Geological Survey Water Investigation 2, 42 p., 11 figs.

----- 1966, Ground water resources of the Battle Creek area, Michigan: Michigan Geological Survey Water Investigation 4, 52 p., 19 figs.

----- 1968, Appendix E of the report on the Grand River Comprehensive Basin Study: U.S. Army Engineers District, Detroit, Michigan, 82 p., 2 figs.

Vanlier, K. E., and Deutsch, Morris, 1958a, Reconnaissance of the groundwater resources of Chippewa County, Michigan: Michigan Geological Survey Progress Report 17, 56 p., 7 figs., 7 pls.

---- 1958b, Reconnaissance of the ground-water resources of Mackinac County, Michigan: Michigan Geological Survey Progress Report 19, 82 p., 8 figs., 6 p1s.

Vanlier, K. E., and Wheeler, M. L., 1968, Analog simulation of ground-water development of the Saginaw Formation, Lansing Metropolitan area, Michigan: Tri-County Planning Commission, Lansing Ground-Water Report, 40 p., 23 figs.

\section{Michigan Water Resources Commission Reports}

Water resources of the Clinton River Basin, 1953.

Water resource conditions and uses in the Paw Paw River Basin, 1955 (revised report in 1964).

Water resources conditions and uses in the Flint River Basin, 1956. in the Huron River Basin, 1957. in the Tittabawassee River Basin, 1960. in the Upper Grand River Basin, 1961. in the Shiawassee River Basin, 1963. in the Maumee River Basin, 1964. in the River Raisin Basin, 1965. in the Au Sable River Basin, 1966. in the Lower Grand River Basin, 1967

(open file). 
Water resources of southeastern Michigan, February 1968.

Water resources of the lower Lake Huron drainage basin, May 1968.

Water quality standards for Michigan intrastate waters, January 1968.

Water quality standards for Michigan waters, Appendix A (interstate and international waters) June 1967.

Water resources uses, present and prospective, and water-quality standards and plan of implementation (revised June 1967) for . . .

Lake Superior and the St. Mary's River,

Lake Huron,

The Menominee and Montreal River basins in Michigan and the other Michigan-Wisconsin interstate boundary waters,

St. Clair River, Lake St. Clair, Detroit River, Lake Erie, and Maumee River basin,

Lake Michigan,

St. Joseph River basin.

Use designation areas for Michigan's intrastate water quality standards, March 1969.

Twenter, F. R., 1966a, General availability and quality of ground water in the bedrock deposits in Michigan: State Resources Planning Division, Michigan Department of Commerce and Michigan Water Resources Commission, map (color).

$1966 \mathrm{~b}$, General availability of ground water in the glacial deposits in Michigan: State Resources Planning Division, Michigan Department of Commerce and Michigan Water Resources Commission, map (color).

\section{ACKNOWLEDGMENTS}

Acknowledgment is made to personnel of Federal and State agencies, county and township governments, industrial concerns, well drillers, consultants, municipalities, and public utilities, without whose cooperation the accumlation of data presented in this report would have not been possible. 
The collection of ground-water data is aided by the following municipalities, institutions, and private organizations:

Cities or villages of Ann Arbor, Battle Creek, Coldwater, Jackson, Kalamazoo, Lansing, Marsha11, Mason, Portage, St. Johns, St. Louis, and Ypsilanti; Ypsilanti Township; counties of Branch, Van Buren, and Kalamazoo; U.S. Army Engineers; Kent Metropolitan Airport; Huron-Clinton Metropolitan Authority; Fisher Body Division of General Motors Corporation; Harman International Industry; Brown Company; Wisconsin-Michigan Power Company; ClevelandCliffs Iron Company; UpJohn Company, and American Aggregates Corporation. 

